\title{
Effective field theory perspective on next-to-minimal composite Higgs models
}

\author{
Yong-Hui Qi®, ${ }^{1,2,3,{ }^{*}}$ Jiang-Hao Yu, ${ }^{4,5, \dagger}$ and Shou-Hua Zhu ${ }^{6,7,1, \sharp}$ \\ ${ }^{1}$ Center for High Energy Physics, Peking University, Beijing 100871, Peoples Republic of China \\ ${ }^{2}$ Asia Pacific Center for Theoretical Physics, Pohang, Gyeongbuk 37673, Korea \\ ${ }^{3}$ Department of Physics, Pohang University of Science and Technology, Pohang, Gyeongbuk 37673, Korea \\ ${ }^{4}$ Institute of Theoretical Physics, Chinese Academy of Sciences, Beijing 100190, Peoples Republic of China \\ ${ }^{5}$ School of Physical Sciences, University of Chinese Academy of Sciences, \\ Beijing 100049, Peoples Republic of China \\ ${ }^{6}$ Department of Physics and State Key Laboratory of Nuclear Physics and Technology, \\ Peking University, Beijing 100871, Peoples Republic of China \\ ${ }^{7}$ Collaborative Innovation Center of Quantum Matter, Beijing 100871, Peoples Republic of China
}

(Received 14 January 2020; accepted 12 December 2020; published 13 January 2021)

\begin{abstract}
We study both the $C P$-even and $C P$-odd effective chiral Lagrangians of a next-to-minimal composite Higgs model, with a symmetry breaking pattern depicted by the coset $S O(6) / S O(5)$ through the sigma/ omega decomposition, in which the Goldstone matrix of the coset is decomposed in terms of the standard model Higgs doublet and an additional scalar singlet $s$ at the electroweak scale. The effective Lagrangians are described by the electroweak chiral operators up to $p^{4}$ order, with a function dependence on the Higgs boson and new scalar $s$, named Higgs function. This function in the effective Lagrangian incorporates the Higgs nonlinearity or vacuum misalignment effects in the next-to-minimal composite Higgs model, leads to various Higgs couplings deviated from the standard model ones, and also indicates the relations among different Higgs couplings in the low energy. Matching to the Higgs effective field theory below the electroweak scale, we obtain various low-energy observables such as the electroweak oblique parameters, anomalous triple and quartic gauge couplings, anomalous couplings of Higgs to gauge bosons, in which the Higgs nonlinearity effects are encoded in the ratio $\xi \equiv v^{2} / f^{2}$ of the electroweak scale and the new physics scale. As a by-product, we obtain a generalized Maurer-Cartan equation sourced by non-Abelian gauge fields. The equation interprets the dynamical interplay between nonlinear realization of pseudo-NambuGoldstone bosons (or Higgs) and the gauge fields, and provides a nonlinear generalization of the dynamical electroweak symmetry breaking.
\end{abstract}

DOI: 10.1103/PhysRevD.103.015013

\section{INTRODUCTION}

The standard model (SM) of elementary particle physics provides a framework for all of the visible matters in nature among the three known fundamental interactions except gravity [1]. The model is described by the gauge theory [2-4] with symmetry $S U(3)_{c} \times S U(2)_{L} \times U(1)_{Y}$. Theoretically the electroweak (EW) sector of the SM still

\footnotetext{
*Corresponding author. yonghui.qi@apctp.org

Corresponding author. jhyu@itp.ac.cn

shzhu@pku.edu.cn

Published by the American Physical Society under the terms of the Creative Commons Attribution 4.0 International license. Further distribution of this work must maintain attribution to the author(s) and the published article's title, journal citation, and DOI. Funded by SCOAP ${ }^{3}$.
}

owns problems such as vacuum stability, too many free parameters, and naturalness or hierarchy problem. Thus, it is commonly believed that the SM is not a UV complete theory at energy above a trillion electron volt (TeV) scale, at where underlying dynamics is still unclear. To solve these problems suffered by the SM, many models beyond the SM are proposed, with different motivations emphasized. Among them, the composite Higgs model (CHM) provides a scenario [5-7], that might be related to a strong dynamics in the high energy, to solve the electroweak hierarchy problem. In the scenario, the Higgs-like particle arises as a pseudo-Nambu-Goldstone boson (PNGB) [8-12] of a global symmetry breaking at a higher energy scale $f$ higher than the EW scale measured by the Higgs vacuum expectation value (vev) $v=2 m_{W} / g=\left(\sqrt{2} G_{F}\right)^{-1 / 2}$ or equivalently the Fermi coupling constant $G_{F}$, and is related with strong dynamical scale $\Lambda_{s} \simeq 4 \pi f$ for an unbroken symmetry as the subgroup of the global symmetry. 
There are many kinds of composite Higgs models in literature; for review, see Refs. [13-16]. We could classify different composite Higgs models based on the numbers and EW representations of GBs in the setup. The most economical and popular composite Higgs model is the $S O(5) / S O(4)$ minimal composite Higgs model (MCHM) [16-20]. The symmetric coset $S O(5) / S O(4)$ provides only four GBs: three GBs eaten by the longitudinal components of the $W$ and $Z$ bosons, and the Higgs boson as the pseudo-Nambu-GB. The next-to-minimal model is the $S U(4) / S p(4) \simeq S O(6) / S O(5)$ next-to-minimal composite Higgs model (NMCHM) [21-26], in which five GBs are presented: four SM Higgs components and an additional singlet scalar. For a heavy singlet scalar GB, this model recovers to the MCHM. Thus the NMCHM contains the MCHM as the limiting case. On the other hand, if this singlet scalar is not so heavy, it provides new low-energy phenomenology, such as dark matter candidate [24,26], electroweak phase transition, etc.

Further classification of the CHMs could be justified using the custodial symmetry. The theory is invariant for the three gauge bosons in the fundamental representation of $S O(3)$ or adjoint representation of $S U(2)_{c}$ [7], and guarantees the $\rho$ parameter, the ratio defined with both mass of gauge bosons and the EW mixing angle as below,

$$
\rho \equiv \frac{m_{W}^{2}}{m_{Z}^{2} c_{\theta}^{2}}=1
$$

at the tree level, although there could be a small correction at the loop level. This custodial protection mechanism in the SM is valid both before and after electroweak symmetry breaking (EWSB), which entails a strong constraint for model building beyond the SM; namely, whatever underlying dynamics as a possible UV completion beyond $\mathrm{TeV}$ scale, its symmetry breaking pattern down to the EW scale, always entails $\rho=1$ at tree level. The electroweak precision tests indicate that we should consider CHMs with the custodial symmetry imposed. Both the MCHM and NMCHM contain the $S O(3)$ group as the subgroup and thus the custodial symmetry is guaranteed.

On the other hand, given the experimental results at the LHC, the lack of evidence of new physics and the precision measurement of the Higgs property have already pushed the new physics scale up to the $\mathrm{TeV}$ scale, unless the new particles do not carry the electroweak charge. In CHMs, the composite particles related to the strong dynamics scale $f$ are typically heavy [13]. If the additional GBs rather than the pseudo-Goldstone Higgs exist, they should not be lighter than the electroweak scale unless the GB is the electroweak singlet. Although there are many kinds of composite Higgs models with different symmetry breaking patterns depicted by the coset $G / H[14,15]$, after integrating out heavy composite states, and heavy GBs, what is left should at least be the SM contents: the matter fields, the SM gauge fields, and the Higgs boson as the pseudo-GB. There could be an additional pseudo-GB being the electroweak singlet. Thus the effective Lagrangian description of the composite Higgs models should contain at least $S O(5) / S O(4)$ symmetry [19]. In this work, we consider a more general case in which an additional light GB exists at the electroweak scale. The typical example is the $S O(6) / S O(5)$ symmetry at the electroweak scale, containing one light scalar singlet GB. ${ }^{1}$ If the scalar singlet GB becomes heavy, it recovers the $S O(5) / S O(4)$ symmetry at the electroweak scale.

From the bottom-up perspective, the low-energy effective field theory (EFT) approaches [27-57] with the general principle of quantum field theory, such as Lorentz invariance, unitary, causality, etc., have provided a universal model-independent description for new physics beyond the SM. There are usually two ways to describe the low-energy effective field theory: the SM EFT in the symmetric phase $[37,42,43]$ and the EW chiral Lagrangian in the broken phase [34,36,38]. For the case that the Higgs boson as the pseudo-Nambu-GBs, the EW chiral Lagrangian is usually adopted due to the Higgs nonlinearity in the scalar manifold of the GB fields. In the EW chiral Lagrangian, the Higgs field is nonlinearly realized, e.g., in the coset construction of Callan-ColemanWess-Zumino (CCWZ) approach [27,28].

It turns out that the phenomenological Lagrangian $[27,28]$ can be used not only to reproduce the soft pions, PNGBs of chiral gauge interactions of $S U(2)_{L} \times S U(2)_{R}$ symmetry results of current algebra [29,30], but also can be used to justify the calculation of soft-pion matrix elements results from the Feynman diagrams, with the advantage of not using any operator algebra. The Lagrangians can be used to calculate corrections to the leading soft pions to any desired order in external momentum. In the spirit of writing down the most general possible Lagrangian terms, it is possible to calculates the most general possible $S$-matrix elements consistent with the general principles of analyticity, perturbative unitary, cluster decomposition, Lorentz invariance, chirality, charge-parity-time symmetry, and the other assumed intrinsic symmetries of quantum field theory (QFT). It is possible to include all possible terms by classifying the relevant operator with the guide of a power-counting argument: the only physical observable quantities are based upon the matrix elements, that must take the form

$$
\mathcal{M}=E^{D} f(E / \mu)
$$

where $E=\sqrt{s}$ is the energy scale related to the center-ofmass energy $s, \mu$ is some arbitrary renormalization scale, of

\footnotetext{
${ }^{1}$ It is worth noticing that not only $S O(5)$ but also $S O(4) \times S O(2)$ is a subgroup of $S O(6)$. For the later case, the symmetry breaking pattern is $S O(6) / S O(4) \times S O(2)$ and there will be eight GBs, which serves as one of possible realizations of the 2-Higgs-doublet model structure, with generators shown in the Appendix. A 1.
} 
which, the matrix elements must be independent, $f(\ldots)$ is an arbitrary dimensionless function, and $D$ is the power counting of the effective Lagrangian operator with the mass dimension [29]

$$
D=2+\sum_{d} V_{d}(d-2)+2 L
$$

where $V_{d}$ is the number of vertices formed from interactions with $d$ derivatives, $L$ is the number of loops in a Feynman diagram, which stands for $L$ independent momentum not fixed by the momentum conservation, e.g., by cutting the $L$ lines simultaneously, the diagram is still connected. The equation can be obtained by using the well-known Feynman-Euler formula for the number of loops in a one-connected graph, which is general for both planar or nonplanar topologies [32]

$$
V-I+L=1,
$$

where $V=\sum_{d} V_{d}$ is the total number of vertices, $I$ is the total number of internal lines, and $L$ is the Feynman loops number. The left-hand side of Eq. (1.4) means there are $V$ delta functions, of which $I-L$ fix internal momentum, and leaving $V-1+L$ delta functions relating the momentum of all incoming or outgoing particles. The right-hand side of the equation implies that there is only a single delta function in a one-connected graph. Thus, this equation encodes the information of momentum conservation at each vertex and implies that the number of vertices that can actually be permitted are limited by the topology of the (Feynman) diagrams. Therefore, according to Eq. (1.3), the lowest possible number of derivatives of the Lagrangian is determined given the spacetime dimension $D$, and the types of graphs, e.g., for tree-/one-loop/two-loop graphs, $L=0$, 1,2 , respectively. Given $D$ is fixed, the power counting of the operators are

(i) $E^{D=2}: L=0, d=2$.

(ii) $E^{D=4}: L=0, d=4 ; L=1, d=2$.

This power counting implies that at $E^{2}$ order, there is only a tree diagram contribution from effective operators with at most two-derivatives terms; at $E^{4}$ order, there are not only tree diagrams contributions from effective operators with at most four-derivatives, but also one-loop diagrams contributions from those operator with at most two derivatives. Similarly, at $E^{6}$ order, in addition to diagrams from treeand one-loop diagrams, there are also two-loop diagrams contributions. The leading graphs are those with the smallest values of $D$. With the power-counting argument, the phenomenological Lagrangians can be used to study symmetry breaking in a systematic approach. The most intuitive one is the "naive dimensional analysis" (NDA) [30], which can provide a qualitative demo on the powercounting approach. With ultraviolet cutoff at $\Lambda=4 \pi f$, the fundamental QFT energy scale can be estimated by the strong dynamical symmetry breaking scale $f,{ }^{2}$ up to which the EFT is valid. The coefficient consistent with NDA is $[30,33]$

$$
\left(\frac{\pi}{f}\right)^{A}\left(\frac{\psi}{f \sqrt{\Lambda}}\right)^{B}\left(\frac{\bar{A}_{\mu}}{\Lambda}\right)^{C}\left(\frac{p}{\Lambda}\right)^{D} f^{2} \Lambda^{2},
$$

where we have neglected a universal factor $(2 \pi)^{4} \delta^{4}\left(\sum_{i} p_{i}\right)$, where $\delta^{4}$ is the $\delta$ function for momentum conservation at the vertex, and $p$ denotes the derivatives acting on either the $\pi$ or $\bar{A}_{\mu}=g A_{\mu}$ fields. $D$ is the number of derivatives acting on external lines. The coefficients are compatible with gauge invariance $D_{\mu}=\partial_{\mu}+i \bar{A}_{\mu}$, and this means each derivative and gauge field $g A_{\mu}$ are associated with coefficients $1 / \Lambda$. The effective field theory based upon the power-counting argument is only phenomenological, in contrast to a fundamental QFT with all input parameters fixed. When graphs involving higher and higher order in PNGBs are calculated, in each successive order, one would encounter more and more undetermined parameters. As a result, practically, the Lagrangian is only calculated up to a given finite order of perturbation theory.

On the other hand, the standard model effective field theory (SMEFT) up to dimensional-six operators cannot fully characterize the high-energy effective Lagrangian up to the $p^{4}$ order, since the operators at this order can also obtain contributions from dimensional-eight operators of SMEFT. Moreover, in the EW chiral Lagrangian, the global $S O(4)$ Higgs singlet $h$ supplies a natural embedding of the custodial symmetry $S U(2)_{C} \sim S O(3) \subset S O(4)$ after the EW symmetry is spontaneously broken

$$
|h|^{2} \rightarrow|\vec{\varphi}|^{2}+(v+h)^{2},
$$

where $\vec{h}=\left(h_{1}, h_{2}, h_{3}, h_{4}\right)$, and $\vec{\varphi}=\left(\varphi_{1}, \varphi_{2}, \varphi_{3}\right)$ consists of the three would-be GBs for the EW symmetry. In the CHM scenerario, again we will specifically focus on those with the $\mathcal{G} / S O(4)$ symmetry breaking pattern. We will take the custodial symmetric CHM as an example to study the nonlinearity of Higgs as well as its possible mixing with a light scalar.

In this work, we take the $S U(4) / S p(4) \simeq S O(6) / S O(5)$ next-to-minimal composite Higgs model [21-26] as an extension of MCHM. In the NMCHM, after the explicit breaking of the global symmetry $f$, the gauge symmetry and the Yukawa terms induce the radiative potential for the SM Higgs, which acquires dynamically a nonvanishing vacuum expectation value

\footnotetext{
${ }^{2}$ The $4 \pi$ factor originates from the one-loop factor $(4 \pi)^{2} \leq$ $f^{2} / \Lambda^{2}$ in four-dimensional QFT. The EFT is invalid at energies higher than $\Lambda$. For composite models, the cutoff scale $\Lambda \approx$ $4 \pi f / \sqrt{N_{\pi}}$ is interpreted as the compositeness scale of GB $\phi$, where $N_{\pi}$ is the number of GBs, since the dynamics of the sigma model is described by a scalar subject to a constraint $\phi^{2}=f^{2}$.
} 


$$
v=f \sin \left(\frac{v_{\phi}}{f}\right) \cos \left(\frac{v_{\psi}}{f}\right)
$$

where $\phi$ denote the quantum fluctuation field around the vacuum with expectation value $v_{\phi}$ and $v_{\psi}$ denoted as the vacuum phase of an extra singlet $s$ relative to the Higgs $h$. In the $f \gg v$ limit, the nonlinear NMCHM should recover the SM limit, in which the EWSB is linearly realized and the Lagrangian is written in terms of the SM Higgs as an $S U(2)_{L}$ doublet.

We connect the low-energy EW chiral Lagrangian to the UV CHM valid up to an energy scale $\Lambda_{s} \sim 4 \pi f$. In the CHMs, according to the CCWZ formalism, the kinetic term of the Higgs boson should originate from [39,58-61]

$$
\mathcal{L}_{2}=f^{2} \operatorname{Tr}\left(d_{\mu} d^{\mu}\right) \supset g_{a b}(h) \partial^{\mu} \pi^{a} \partial_{\mu} \pi^{a},
$$

where the metric $g_{a b}(h)$ parametrizes the curvature in the scalar manifold. Given the nonflat metric, the degree of nonlinearity of the theory can be quantified by the nonlinear parameter

$$
\xi \equiv \frac{v^{2}}{f^{2}}
$$

which recovers the flat metric if $f \rightarrow \infty$.

On the other hand, the Wilson coefficients of the effective operators in the EW chiral Lagrangian can be described by the Higgs functions (or radial functions) $\mathcal{F}_{H}$ $[49,52,60]$, as

$$
\mathcal{L}_{h}=c_{H} \mathcal{F}_{H}(h, s) \mathcal{L}_{H}+\ldots,
$$

where $\mathcal{L}_{H}$ is defined in Eq. (C9), ... denote the higher order terms, e.g., at the $p^{4}$ order, including the $C P$-even $\mathcal{L}_{C, T}$ or $C P$-odd $\mathcal{L}_{\tilde{T}}$ defined in Eqs. $(\mathrm{C} 10)$ and (C14) in Appendix. C. The Higgs functions encode all the information of the Higgs nonlinearity due to the nonflat metric of pseudo-Nambu-GBs manifold of the CHM.

The Higgs functions provide the connection between the low-energy EW EFT and the chiral Lagrangian for the CHM. The effective Lagrangian of the model matches with the EW chiral effective Lagrangian up to the $p^{4}$ order. We extract the Higgs functions of the model from the lowenergy EW chiral Lagrangian, which contains information of not only the Higgs itself but also that of an additional light scalar $s$. In a series expansion of $h / v$ in the Higgs effective field theory (HEFT) with the parameter $\xi$ fixed, we find the effective Wilson coefficients associated with the high-energy effective chiral Lagrangian in the NMCHM. Observables such as the EW oblique parameters, anomalous triple and quartic gauge couplings, and anomalous couplings of Higgs to gauge bosons are obtained.

The structure of the paper is organized as follows: In Sec. II, we study the building blocks for both Sigma and
Omega representation in the NMCHM and discuss its symmetries. We also illuminate the significance of Higgs nonlinearity. In Sec. III, we study high-energy chiral effective Lagrangian of $\mathrm{NMCHH}$ up to the $p^{4}$ order. In Sec. IV, we match the chiral effective Lagrangian of the NMCHM with low-energy effective EW chiral Lagrangian (EWCL), and extract the Higgs functions. In Sec. V, we study the Higgs function at EW scale. In Sec. VI, we study the connection of effective field theory to the corresponding physical observables. Conclusions are made in Sec. VII.

\section{NEXT-TO-MINIMAL COMPOSITE HIGGS MODEL}

In generic composite Higgs $(\mathrm{CH})$ scenery, a global symmetry group $\mathcal{G}$ is spontaneously broken by some strong dynamics mechanism at the scale $f$ down to a subgroup $\mathcal{H}$. The coset $\mathcal{G} / \mathcal{H}$ is assumed symmetric and entails that $\operatorname{dim}(\mathcal{G} / \mathcal{H}) \geq 4$, e.g., the minimal version in terms of minimal composite Higgs model (MCHM) [17]. Consequently, there are (at least) four GBs that arise from the nonlinear symmetry breaking mechanism of the global symmetry $\mathcal{G}$; one is identified with the light Higgs-like scalar field $h$ and three are identified with the longitudinal components of the SM gauge bosons.

Following CCWZ in Appendix. B, we can introduce the Goldstone field matrix $\Omega$, which transform nonlinearly under the group $\mathcal{G}$. In the symmetric coset case, it is equivalent to use either $\Omega$ or $\boldsymbol{\Sigma}$ parametrization in describing the GBs degrees of freedoms and its interactions. To obtain gauge interactions by formally gauging the symmetry $\mathcal{G}$, one has introduced the $\mathcal{G}$ gauge fields strength tensor $\overline{\mathrm{A}}_{\mu}$ as introduced Eq. (B15) that satisfies the gauge transformations in Eq. (B15). The EW gauge field $\overline{\mathrm{W}}_{\mu}$ and $\overline{\mathrm{B}}_{\mu}$ are embedded as $\left(\overline{\mathrm{W}}_{\mu}, \overline{\mathrm{B}}_{\mu}\right) \subset \overline{\mathrm{A}}_{\mu} \in \mathcal{G}$ with

$$
\overline{\mathrm{W}}_{\mu} \equiv W_{\mu}^{a} Q_{L}^{a} \quad \text { and } \quad \overline{\mathrm{B}}_{\mu} \equiv B_{\mu} Q_{Y}
$$

$Q_{L}^{a}$ and $Q_{Y}$ denote the embedding in $\mathcal{G}$ of the $S U(2)_{L} \times$ $U(1)_{Y}$ generators. The $\mathcal{G}$ gauge field $\overline{\mathrm{A}}_{\mu} \in \mathcal{G}$ can be doubled into two copies $\overline{\mathrm{W}}_{\mu}$ and $\overline{\mathrm{B}}_{\mu}$ by taking account of its automorphism field strength tensor [49,52].

To construct the nonlinear chiral Lagrangian, different building blocks in the last section are utilized. The building blocks for the Sigma parametrization in the NMCHM are

$$
\overline{\mathrm{W}}_{\mu \nu}, \quad \overline{\mathrm{B}}_{\mu \nu}, \quad \boldsymbol{\Sigma}, \quad \overline{\mathrm{V}}_{\mu}, \quad \overline{\mathbf{T}},
$$

where the corresponding gauge fields $\overline{\mathrm{W}}_{\mu}$ and $\overline{\mathrm{B}}_{\mu}$ are defined in Eq. (2.1). For the gauged version of the nonlinear sigma model, the chiral vector field $\overline{\mathrm{V}}_{\mu}$ and the scalar chiral field $\overline{\mathbf{T}}$ are defined in Eqs. (B34) and (B38), respectively.

For the next-to-minimal composite Higgs model with the coset $S O(6) / S O(5)$, there is an additional new singlet GB $s$, compared with those in the MCHM. For a symmetric 
coset, equivalently the $\boldsymbol{\Sigma}$ field could be introduced, transforming linearly under the group $\mathcal{G}$. $Q_{L}^{a}$ with $a=1,2,3$ and $Q_{Y}$ are the $S U(2)_{L} \times U(1)_{Y}$ generators embedded in $S O(6)$ as given in Appendix. A

$$
Q_{L}^{a} \equiv T_{L}^{a}, \quad Q_{Y} \equiv T_{R}^{3},
$$

where we have identified the $S U(2)_{L}$ charge operator as $T_{L}^{a}$ and the hypercharge operator as $T_{R}^{3}$ as defined in Eq. (A14). When fermions are taken into account, the realistic hypercharge operator is defined as $Y=T_{R}^{3}+X$, where $X$ is a new nonvanishing charge under an additional $U(1)_{X}$, in order to reproduce the correct hypercharges of fermions.

\section{A. Goldstone boson field matrix}

The GBs of the coset $S O(6) / S O(5)$ in the fundamental representation can be parametrized by ${ }^{3}$

$$
\Phi\left(h_{\hat{a}}\right)=\Omega \Phi_{0}, \quad \Phi_{0}=\left(\begin{array}{c}
0_{5} \\
1
\end{array}\right),
$$

where $\Phi_{0}$ is the vacuum expectation value in the fundamental representation of $S O(6)$ as a six-dimensional unit vector.

\section{Omega parametrization}

In the symmetric coset $S O(6) / S O(5)$, it is equivalent to use the $\Omega$ or $\boldsymbol{\Sigma}$ to describe the Goldstone degree of freedom (d.o.f.) as

$$
\Omega=e^{i \frac{\sqrt{2}}{f} T^{\hat{a}} h \hat{a}} \equiv e^{i \Pi,} \quad \boldsymbol{\Sigma}=\Omega^{2}=e^{2 i \Pi},
$$

where $T^{\hat{a}}$ are the generators in the coset $S O(6) / S O(5)$ as given in Eq. (A3) in Appendix. A, where $\hat{a}=(\hat{\alpha}, 5)$ with $\hat{\alpha}=1,2,3,4 . T^{\hat{\alpha}}$ are the unbroken generators of $S O(4)$ and $T^{\hat{5}}$ is that of $S O(2)$. The first four indices span a fourparameter coset space and $h_{\hat{\alpha}}$ is an $S O(4)$ vector. Denoting the GBs in an array

$$
\hat{\phi} \equiv\left(\hat{h}_{\hat{\alpha}}, \hat{h}_{5}\right)^{T}, \quad \text { with } \quad \phi=\sqrt{h_{\hat{a}} h_{\hat{a}}}
$$

where $\hat{h}_{\hat{\alpha}} \equiv\left(\hat{h}_{1}, \hat{h}_{2}, \hat{h}_{3}, \hat{h}_{4}\right)^{T}$ and we have $\hat{h}_{\hat{a}} \equiv h_{\hat{a}} / \phi$. The Goldstone boson field matrix is expressed as

$\Pi=\frac{\phi}{f} \Xi, \quad$ with $\quad \Xi=\sqrt{2} T^{\hat{a}} \hat{h}_{\hat{a}}=-i\left(\begin{array}{c|c}\mathbf{0}_{5 \times 5} & \hat{\phi} \\ \hline-\hat{\phi}^{T} & 0\end{array}\right)$.

\footnotetext{
${ }^{3}$ We have introduced $\Phi_{0}$ to be dimensionless, so that the kinetic terms should be defined as $\mathcal{L}_{\text {kin }}=f^{2}\left(\partial_{\mu} \Phi\right)^{T}\left(\partial^{\mu} \Phi\right) / 2$. Alternatively, one may define $\Phi_{0}=\left(0_{5}, f\right)^{T}$ with a dimensional scale $\langle f\rangle$; in this case, the kinetic terms should be defined as in Eq. (5.8)
}

It is convenient to define the mixing angle $\phi$ at strong dynamics breaking scale $f$ as

$$
s_{\phi} \equiv \sin \left(\frac{\phi}{f}\right), \quad c_{\phi} \equiv \cos \left(\frac{\phi}{f}\right)
$$

Thus, one can express the Goldstone matrix $\Omega$ as

$$
\Omega=\left(\begin{array}{c|c}
1-\left(1-c_{\phi}\right) \hat{\phi} \hat{\phi}^{T} & s_{\phi} \hat{\phi} \\
\hline-s_{\phi} \hat{\phi}^{T} & c_{\phi}
\end{array}\right),
$$

where

$$
\hat{\phi} \hat{\phi}^{T}=\left(\begin{array}{cc}
\hat{h} \hat{h}^{T} & \hat{h}_{5} \hat{h} \\
\hat{h}_{5} \hat{h}^{T} & \hat{h}_{5}^{2}
\end{array}\right)
$$

and $\hat{\phi}^{T} \hat{\phi}=\operatorname{Tr}\left(\hat{h} \hat{h}^{T}\right)+\hat{h}_{5}^{2}=1$.

After the electroweak symmetry is broken, in the unitary gauge, i.e., $\hat{h}_{1}=\hat{h}_{2}=\hat{h}_{3}=0$, it is convenient to define another angle as [25]

$$
\begin{gathered}
c_{\psi} \equiv \cos \left(\frac{\psi}{f}\right)=\frac{h_{4}}{\sqrt{h_{4}^{2}+h_{5}^{2}}}, \\
s_{\psi} \equiv \sin \left(\frac{\psi}{f}\right)=\frac{h_{5}}{\sqrt{h_{4}^{2}+h_{5}^{2}}},
\end{gathered}
$$

where $\psi=\arctan \left(h_{5} / h_{4}\right)$ is pseudoscalar under $C P$ symmetry due to the relative phase between the $h_{5}$ and $h_{4}$. In the unitary gauge, the amplitude $\phi$ reduces to $\phi=\sqrt{h_{4}^{2}+h_{5}^{2}}$ and the $\left(h_{4}, h_{5}\right)$ can be expressed in terms of $\phi$ and $\psi$ as

$$
h_{4}=\phi c_{\psi}, \quad h_{5}=\phi s_{\psi},
$$

Thus, the GB matrix $\Omega$ in Eq. (2.9) can be reexpressed as

$$
\Omega=\left(\begin{array}{cccc}
\mathbf{1}_{3} & 0 & 0 & 0 \\
0 & c_{\phi} c_{\psi}^{2}+s_{\psi}^{2} & \left(c_{\phi}-1\right) c_{\psi} s_{\psi} & c_{\psi} s_{\phi} \\
0 & \left(c_{\phi}-1\right) c_{\psi} s_{\psi} & c_{\psi}^{2}+c_{\phi} s_{\psi}^{2} & s_{\psi} s_{\phi} \\
0 & -c_{\psi} s_{\phi} & -s_{\phi} s_{\psi} & c_{\phi}
\end{array}\right)
$$

In the original Cartesian $\left(h_{4}, h_{5}\right)$ basis, one has 


$$
\Omega=\left(\begin{array}{cccc}
1_{3} & 0 & 0 & \\
0 & \frac{h_{4}^{2} c_{\phi}+h_{5}^{2}}{h_{4}^{2}+h_{5}^{2}} & \frac{h_{4} h_{5}\left(c_{\phi}-1\right)}{h_{4}^{2}+h_{5}^{2}} & \frac{h_{4} s_{\phi}}{\sqrt{h_{4}^{2}+h_{5}^{2}}} \\
0 & \frac{h_{4} h_{5}\left(c_{\phi}-1\right)}{h_{4}^{2}+h_{5}^{2}} & \frac{h_{5}^{2} c_{\phi}+h_{4}^{2}}{h_{4}^{2}+h_{5}^{2}} & \frac{h_{5} s_{\phi}}{\sqrt{h_{4}^{2}+h_{5}^{2}}} \\
0 & -\frac{h_{4} s_{\phi}}{\sqrt{h_{4}^{2}+h_{5}^{2}}} & -\frac{h_{5} s_{\phi}}{\sqrt{h_{4}^{2}+h_{5}^{2}}} & c_{\phi}
\end{array}\right) .
$$

For the latter convenience of relating the $h_{4,5}$ to the SM Higgs $h$, we introduce a new basis $(h, s)$ as

$$
\begin{aligned}
& \frac{h}{f} \equiv \frac{h_{4}}{\sqrt{h_{4}^{2}+h_{5}^{2}}} \sin \left(\frac{\sqrt{h_{4}^{2}+h_{5}^{2}}}{f}\right) \stackrel{f \rightarrow \infty}{=} \frac{h_{4}}{f}, \\
& \frac{s}{f} \equiv \frac{h_{5}}{\sqrt{h_{4}^{2}+h_{5}^{2}}} \sin \left(\frac{\sqrt{h_{4}^{2}+h_{5}^{2}}}{f}\right) \stackrel{f \rightarrow \infty}{=} \frac{h_{5}}{f}
\end{aligned}
$$

in the weak coupling limit, i.e., $f \rightarrow \infty,(h, s)$ just recovers $\left(h_{4}, h_{5}\right)$, respectively. One can check that

$$
\sin ^{2}\left(\frac{\sqrt{h_{4}^{2}+h_{5}^{2}}}{f}\right)=\frac{h^{2}+s^{2}}{f^{2}}, \quad \frac{h_{4}}{h_{5}}=\frac{h}{s},
$$

and the phase $\psi$ also represents the relative phase between the singlet $s$ and Higgs $h$ as

$$
t_{\psi}=\tan \left(\frac{s}{h}\right)
$$

With Eqs. (2.8) and (2.11), the $(h, s)$ fields can be expressed in terms of $(\phi, \psi)$ as

$$
h=f c_{\psi} s_{\phi}, \quad s=f s_{\psi} s_{\phi} .
$$

The amplitude $\phi=\sqrt{h^{2}+s^{2}}$ is a scalar and consists of $h$ and $s$. In the $(h, s)$ basis, the GBs matrix in Eq. (2.13) can be expressed as

$\Omega=\left(\begin{array}{cccc}1_{3} & 0 & 0 & 0 \\ 0 & \frac{h^{2} \sqrt{1-\frac{h^{2}+s^{2}}{f^{2}}}+s^{2}}{h^{2}+s^{2}} & \frac{h s\left(\sqrt{1-\frac{h^{2}+s^{2}}{f^{2}}}-1\right)}{h^{2}+s^{2}} & \frac{h}{f} \\ 0 & \frac{h s\left(\sqrt{\left.1-\frac{h^{2}+s^{2}}{f^{2}}-1\right)}\right.}{h^{2}+s^{2}} & \frac{s^{2} \sqrt{1-\frac{h^{2}+s^{2}}{f^{2}}}+h^{2}}{h^{2}+s^{2}} & \frac{s}{f} \\ 0 & -\frac{h}{f} & -\frac{s}{f} & \sqrt{1-\frac{h^{2}+s^{2}}{f^{2}}}\end{array}\right)$.

Finally let us consider the situation in the absence of a $C P$-odd singlet, in which the NMCHM just recovers that of MCHM, with

$$
\frac{h}{f} \equiv \sin \left(\frac{h_{4}}{f}\right) \stackrel{f \rightarrow \infty}{=} \frac{h_{4}}{f}, \quad s=0 .
$$

In the $(\phi, \psi)$ basis, with $\psi=0$, the GB matrix in Eq. (2.13) recovers that for MCHM in $S O(5) \subset S O(6)$ as

$$
\Omega=\left(\begin{array}{cccc}
\mathbf{1}_{3} & 0 & 0 & 0 \\
0 & c_{\phi} & 0 & s_{\phi} \\
0 & 0 & 1 & 0 \\
0 & -s_{\phi} & 0 & c_{\phi}
\end{array}\right) .
$$

Equivalently, in the original Cartesian $\left(h_{4}, h_{5}\right)$ basis with $h_{4} \equiv h, h_{5}=0$, it is just recovered as

$$
\Omega=\left(\begin{array}{cccc}
1 & 0 & 0 & 0 \\
0 & \cos \left(\frac{h}{f}\right) & 0 & \sin \left(\frac{h}{f}\right) \\
0 & 0 & 1 & 0 \\
0 & -\sin \left(\frac{h}{f}\right) & 0 & \cos \left(\frac{h}{f}\right)
\end{array}\right) .
$$

While also equivalently, in the $(h, s)$ basis, with $s=0$, the GBs matrix becomes

$$
\Omega=\left(\begin{array}{cccc}
1_{3} & 0 & 0 & 0 \\
0 & \sqrt{1-\frac{h^{2}}{f^{2}}} & 0 & \frac{h}{f} \\
0 & 0 & 1 & 0 \\
0 & -\frac{h}{f} & 0 & \sqrt{1-\frac{h^{2}}{f^{2}}}
\end{array}\right),
$$

which gives the exponential nonlinear parametrization of $S O(5)$ transformation on GBs in terms of unconstrained coordinates $\left(h_{1}, h_{2}, h_{3}, h_{4}\right) \in S O(4)$.

\section{From Omega to Sigma parametrization}

For symmetric coset, the GB matrix can be also parametrized as

$$
\boldsymbol{\Sigma}=\Omega^{2},
$$

which can be obtained from $\Omega$ in Eqs. (2.13), by making the replacement as

$$
f \rightarrow \frac{f}{2}, \quad \psi \rightarrow \frac{1}{2} \psi,
$$

so that one needs to make the replacement as

$$
\begin{aligned}
& c_{\phi} \rightarrow c_{2 \phi}, \quad s_{\phi} \rightarrow s_{2 \phi}, \\
& c_{\psi} \rightarrow c_{\psi}, \quad s_{\psi} \rightarrow s_{\psi},
\end{aligned}
$$

and in the $(h, s)$ basis, equivalent to making the replacement 
TABLE I. Fields coordinate transformation among $(\phi, \psi),\left(h_{4}, h_{5}\right)$, and $(h, s)$ for $\Omega$ parametrization.

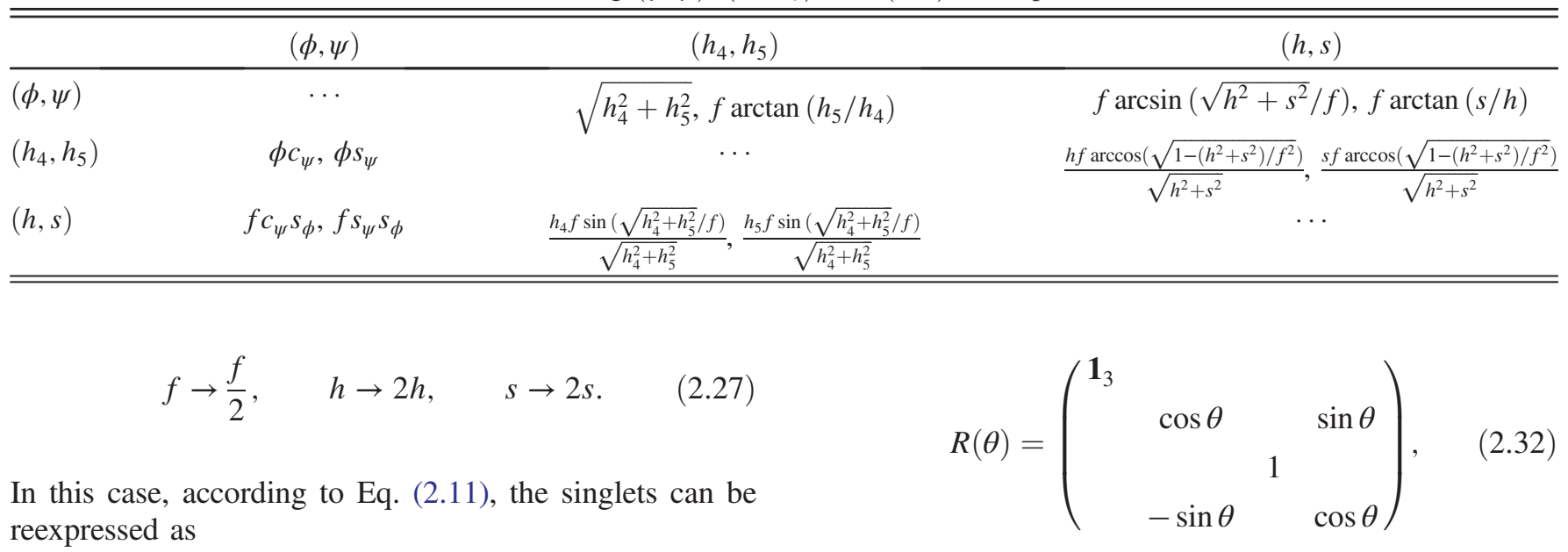

$$
(h, s)=\frac{\sin \left(\frac{n}{f} \phi\right)}{\left(\frac{n}{f}\right)}\left(c_{\psi}, s_{\psi}\right), \stackrel{f \rightarrow \infty}{=}\left(h_{4}, h_{5}\right),
$$

from which, one can reexpress the phase $\phi$ and $\psi$, respectively, in terms of singlets $h$ and $s$ as

$$
\begin{aligned}
& \phi=\frac{f}{n} \arcsin \left(\frac{n}{f} \sqrt{h^{2}+s^{2}}\right)=\sqrt{h_{4}^{2}+h_{5}^{2}}, \\
& \psi=f \arctan \left(\frac{s}{h}\right)=f \arctan \left(\frac{h_{5}}{h_{4}}\right),
\end{aligned}
$$

where $n=1$ for $\Omega$ parametrization and $n=2$ for $\boldsymbol{\Sigma}$ parametrization, respectively. This implies that for $\boldsymbol{\Sigma} \equiv \Omega^{n}$, one just makes the replacement

$$
f \rightarrow \frac{f}{n}, \quad \psi \rightarrow \frac{\psi}{n},
$$

so that the ratio $s / h$ is unchanged. For the convenience of later usage, we summarize the fields coordinate transformation among $(\phi, \psi),\left(h_{4}, h_{5}\right),(h, s)$ in Table. I.

\section{B. Symmetries}

\section{Rotational symmetry}

One may rotate the generators of unbroken $S O(4) \subset$ $S O(5)$ in Eqs. (A14) as well as the coset generator $S O(5) / S O(4)$ in Eq. (A3) by an angle $\theta$ in the $S O(5)$ inner space,

$$
T^{\alpha} \equiv\left(T_{L}^{a}, T_{R}^{a}, T^{\hat{a}}\right) \rightarrow T^{\alpha \prime} \equiv R(\theta) T^{\alpha} R^{-1}(\theta)
$$

by a rotation matrix $R(\theta)$ where $\mathbf{1} \equiv \mathbf{1}_{3 \times 3}$. The angle $\theta$ parametrizes the misalignment of $S O(6)$ vacuum as we will discuss in the following section. It turns out that the generators rotated become

$$
\begin{aligned}
& T_{L}^{a}(\theta)=\frac{1+c_{\theta}}{2} T_{L}^{a}+\frac{1-c_{\theta}}{2} T_{R}^{a}-\frac{s_{\theta}}{\sqrt{2}} T^{\hat{a}}, \\
& T_{R}^{a}(\theta)=\frac{1-c_{\theta}}{2} T_{L}^{a}+\frac{1+c_{\theta}}{2} T_{R}^{a}+\frac{s_{\theta}}{\sqrt{2}} T^{\hat{a}}, \\
& T^{\hat{a}}(\theta)=\frac{s_{\theta}}{\sqrt{2}} T_{L}^{a}-\frac{s_{\theta}}{\sqrt{2}} T_{R}^{a}+c_{\theta} T^{\hat{a}}, \quad \hat{a}=1,2,3, \\
& T^{\hat{4}}(\theta)=T^{\hat{4}}, \quad T^{\hat{5}}(\theta)=c_{\theta} T^{\hat{5}}-s_{\theta} T^{4},
\end{aligned}
$$

where $s_{\theta} \equiv \sin \theta$, and $c_{\theta} \equiv \cos \theta, T^{\hat{5}}(\theta)$ is the generator of $S O(2)$ as defined in Eq. (A4) in Appendix. A.

It can be checked that all the generators are also normalized as

$\operatorname{Tr}\left[T_{L}^{a}(\theta) T_{L}^{b}(\theta)\right]=\delta^{a b}, \quad \operatorname{Tr}\left[T_{R}^{a}(\theta) T_{R}^{b}(\theta)\right]=\delta^{a b}$,

$\operatorname{Tr}\left[T^{\hat{a}}(\theta) T^{\hat{b}}(\theta)\right]=\delta^{\hat{a} \hat{b}}=2\left(T^{\hat{a}} T^{\hat{b}}\right)_{66}$,

and one can also check that $\left(T_{L, R}^{a} T^{\hat{b}}\right)_{66}=\left(T^{\hat{a}} T_{L, R}^{b}\right)_{66}=$ $\left(T_{L, R}^{a} T_{L, R}^{b}\right)_{66}=\left(T_{L, R}^{a} T_{R, L}^{b}\right)_{66}=0$. From the generator with rotation angle $\theta$, one can read that the components of the external gauge fields are given by

$$
\begin{gathered}
A_{\mu}^{a}(\theta): A_{\mu}^{a L}(\theta)=\frac{1+c_{\theta}}{2} W_{\mu}^{a}+\frac{1-c_{\theta}}{2} B_{\mu} \delta^{a 3}, \\
A_{\mu}^{a R}(\theta)=\frac{1-c_{\theta}}{2} W_{\mu}^{a}+\frac{1+c_{\theta}}{2} B_{\mu} \delta^{a 3}, \\
A_{\mu}^{\hat{a}}(\theta): A_{\mu}^{\hat{a}}(\theta)=\frac{s_{\theta}}{\sqrt{2}}\left(W_{\mu}^{\hat{a}}-\delta^{\hat{a} 3} B_{\mu}\right), \quad A_{\mu}^{\hat{4}}=0,
\end{gathered}
$$

where $W_{\mu}^{a}, B_{\mu}$ are the EW $S U(2)_{L} \times U(1)_{Y}$ vector bosons. 


\section{Automorphism symmetry}

Since the quotient space $S O(6) / S O(5)$ is symmetric, the symmetric coset has a automorphism or "grading" symmetry that acts upon the generators of $\mathcal{G}$ as defined in Eq. (B5) or (2.37); both lead to the same representation as

$$
\mathcal{R}:\left\{\begin{array}{l}
T_{a}(\theta) \rightarrow+T_{a}(\theta) \\
T_{\hat{a}}(\theta) \rightarrow-T_{\hat{a}}(\theta) .
\end{array}\right.
$$

It is a linear transformation among the generators that preserves the algebra. There is other automorphism given by [23]

$$
\mathcal{R}_{2}:\left\{\begin{array}{l}
T_{a}(\theta) \rightarrow-T_{a}^{T}(\theta) \\
T_{\hat{a}}(\theta) \rightarrow T_{\hat{a}}^{T}(\theta)
\end{array}\right.
$$

The two linear transformations preserve the Lie algebra following from the fact that the $S O(6) / S O(5)$ is symmetric space. Both these two automorphisms lead to the same representation as Eq. (2.38).

With the generators in Eq. (2.33), the automorphism symmetry of $S O(6) / S O(5)$ in Eq. (2.36) is

$$
\begin{gathered}
\mathcal{R}(\theta)=\left(\begin{array}{cccc}
\mathbf{1}_{3 \times 3} & 0 & 0 & 0 \\
0 & \cos (2 \theta) & 0 & -\sin (2 \theta) \\
0 & 0 & 1 & 0 \\
0 & -\sin (2 \theta) & 0 & -\cos (2 \theta)
\end{array}\right) \\
\stackrel{\theta=0, \pi}{=} \operatorname{diag}(1,1,1,1,1,-1),
\end{gathered}
$$

which satisfies

$$
\begin{aligned}
\mathcal{R}(\theta) T_{L, R}^{a}(\theta) \mathcal{R}^{-1}(\theta) & =T_{L, R}^{a}(\theta), \\
\mathcal{R}(\theta) T_{\hat{a}}(\theta) \mathcal{R}^{-1}(\theta) & =-T_{\hat{a}}(\theta),
\end{aligned}
$$

where $a=1,2,3$ and $\hat{a}=1,2,3,4,5$.

When the $S U(2)_{L} \times U(1)_{Y}$ symmetry is turned on, the grading symmetry $\mathcal{R}$ is explicitly broken to

$$
\theta=0, \pi
$$

Since $\mathcal{R}$ is an element of the unbroken $S O(4)$ symmetry, i.e., it is an internal automorphism of the algebra, it will be an exact symmetry of the low-energy Lagrangian up to any order in the absence of gauging the EW symmetry.

It is also interesting that when $\theta=\pi / 2$, the automorphism corresponds to the Higgs parity as [62]

$$
\mathcal{R}(\theta) \stackrel{\theta=\pi / 2}{=} \operatorname{diag}(1,1,1,-1,1,1) \equiv P_{H},
$$

which transforms the generators as
$T^{A}=\left\{T_{L}^{a}, T_{R}^{a}, T^{\hat{a}}, T^{\hat{4}}\right\} \rightarrow T^{\prime A}=\left\{T_{R}^{a}, T_{L}^{a}, T^{\hat{a}},-T^{\hat{4}}\right\}$,

implying that the operator $P_{H}$ flips the direction of $T^{\hat{4}}$ through

$$
T^{A}=P_{H} T^{A} P_{H}^{-1} .
$$

\section{Left-right parity symmetry}

There is also a left-right parity symmetry $P_{L R}$ in the NMCHM,

$$
P_{L R}=\operatorname{diag}(1,1,1,-1,1,-1),
$$

which exchanges the generators of $S U(2)_{L}$ and $S U(2)_{R}$ subgroup of $S O(4)$, and also changes the sign of the first three broken generators $T^{\hat{a}}$ as

$$
\begin{aligned}
P_{L R} T_{L, R}^{a} P_{L R}^{-1} & =T_{R, L}^{a}, \\
P_{L R} T^{\hat{a}} P_{L R}^{-1} & =-T^{\hat{a}}, \\
P_{L R} T^{\hat{4}} P_{L R}^{-1} & =T^{\hat{4}},
\end{aligned}
$$

with $a=1,2,3$ and $\hat{a}=1,2,3,5$. For the broken generators, one should be rewritten as $P_{L R} T^{\hat{a}} P_{L R}^{-1}=$ $-\eta^{\hat{a}} T^{\hat{a}}$ with $\eta^{\hat{a}}=(1,1,1,-1)^{T}$. Since it is not an element of $S O(4)$, one would expect it broken at $p^{4}$ order, although it is an accidental symmetry of GBs Lagrangian at $\left(p^{2}\right)$. When the SM gauge symmetry $S U(2)_{L} \times U(1)$ is turned on, $P_{L R}$ is explicitly broken for a generic value of $\theta$.

\section{CP symmetry}

The $C P$ symmetry is a symmetry of the sigma model of the Higgs sector in the $S O(6) / S O(5)$ model [23]. The first automorphism symmetry $\mathcal{R}$ in Eq. (2.36) makes the vielbein $\overline{\mathrm{V}}_{\mu}=\left(D_{\mu} \boldsymbol{\Sigma}\right) \boldsymbol{\Sigma}^{-1}$ change the sign, so the WessZumino-Witten (WZW) term

$$
\mathcal{L}_{\mathrm{WZW}}=\epsilon^{\mu \nu \rho \sigma \tau} \operatorname{Tr}\left[\overline{\mathrm{V}}_{\mu} \overline{\mathrm{V}}_{\nu} \overline{\mathrm{V}}_{\rho} \overline{\mathrm{V}}_{\sigma} \overline{\mathrm{V}}_{\tau}\right]
$$

changes the sign. While the second automorphism symmetry $\mathcal{R}_{2}$ in Eq. (2.37) does not make the vielbein $\overline{\mathrm{V}}_{\mu}=$ $\left(D_{\mu} \boldsymbol{\Sigma}\right) \boldsymbol{\Sigma}^{-1}$ change the sign, so that it is a symmetry of the WZW term.

The WZW term is unchanged under two conditions

$$
\mathcal{R} P_{0}, \quad \mathcal{R}_{2},
$$

where

$$
P_{0}: x \rightarrow-x
$$


is the spacetime parity. In the $\mathrm{NMCHM}, \mathcal{R}_{2} P_{0}$ corresponds to

$$
h \rightarrow h, \quad s \rightarrow-s,
$$

which defines the $C P$ symmetry of the Higgs sector, including the WZW term. In this case, one can write down a gauge invariant Lagrangian of the form

$$
\mathcal{L}=\frac{s}{(4 \pi)^{2}}\left(n_{B} B_{\mu \nu} \tilde{B}_{\mu \nu}+n_{W} W_{\mu \nu} \tilde{W}_{\mu \nu}+n_{G} G_{\mu \nu} \tilde{G}_{\mu \nu}\right),
$$

where $\tilde{B}^{\mu \nu}=\epsilon^{\mu \nu \rho \sigma} B_{\rho \sigma} / 2$ and $n_{B, W, G}$ are integers that measure the strengths of the gauge anomalies, which are fixed by the fermion content in the UV.

\section{Vacuum misalignment}

The electroweak symmetry should be broken at the electroweak scale. It has been shown that the EW symmetry breaking can be viewed as due to the misalignment angle $\theta$ with respect to the vacuum of $S O(6)$. Even assuming there is no misalignment at the tree level, a nonvanishing $\theta=$ $\langle h\rangle / f$ is generated at the loop level after the GBs obtain a vev $\langle h\rangle=v$, becoming PNGBs. There are two energy scales $f$ and $v$, and the EWSB can be described as a twostep process: at first, $S O(6)$ is spontaneously broken down to $S O(5)$ at the scale $f$, giving rise to an $S U(2)_{L}$ doublet and a singlet of GBs [15]; second, the EW symmetry is spontaneously broken from $S O(4)$ down to $S O(3)$ at the EW scale, which is defined as below, leaving an approximate custodial symmetry as

$$
v=f \sin \left(\frac{\left\langle h_{4}\right\rangle}{f}\right) \equiv f \sin \theta,
$$

where in the last equality, the vev is related to the misalignment of $S O(6)$ vacuum, parametrized by the rotation angle $\theta$; this will become clear as shown in Eq. (2.70). In general, when the singlet $s$ in NMCHM also obtains nonvanishing vevs $v_{s}=\langle s\rangle$, the quality above needs to be slightly modified and the exact formula becomes Eq. (5.32).

Therefore, the vacuum misalignment parameter $\theta$ is related to the nonlinear parameter as

$$
\sqrt{\xi}=\sin \theta \stackrel{\langle h\rangle \gg f}{\approx} \theta .
$$

\section{Embedding of $\mathrm{SO}(4)$ symmetry}

Here we study the correspondence between the $S O(6) /$ $S O(5)=S^{5}$ PNGBs in the NMCHM and the SM Higgs. It it convenient to reparametrize the first three components $h_{a}$ as the three massless SM GBs $\varphi_{a}$ with $a=1,2,3$,

$$
\hat{h}_{a} \equiv \frac{h}{v} \varphi_{a}
$$

and define the mixing angle $\varphi$ as

$$
s_{\varphi} \equiv \sin \left(\frac{|\varphi|}{v}\right), \quad c_{\varphi} \equiv \cos \left(\frac{|\varphi|}{v}\right)
$$

with absolute value $|\varphi|=\sqrt{\varphi^{a} \varphi^{a}}$.

In the following, the first four elements of the GB scalar with $S O(4)$ symmetry are embedded into a six-dimensional fundamental scalar in $S O(6)$. We can rewrite the first four components as a 4-vector times a phase factor $\mathbb{U}$ as embedding of $\mathbf{U}$ in Eq. (A37) into $S O(6)$ as

$$
\mathbb{U} \equiv \exp \left(i \frac{\varphi^{a}}{v} t_{L}^{a}\right)=c_{\varphi}+i \hat{\varphi}^{a} t_{L}^{a} s_{\varphi},
$$

where $t_{L}^{a}=2 T_{L}^{a}$ with $T_{L}^{a}$ are $S O(6)$ embedding generators of custodial symmetry $S U(2)_{L} \simeq S O(3) \subset S O(4)$. One may define the GBs as

$$
\pi^{a} \equiv v s_{\varphi} \hat{\varphi}^{a},
$$

so that the unitary matrix can be reexpressed in analogy to Eq. (A54) as

$$
\mathbb{U}=\sqrt{1-\frac{\pi^{2}}{v^{2}}}+i \frac{\pi^{a}}{v} t_{L}^{a},
$$

which defines the coordinate change.

In terms of the phase factor $\mathbb{U}$, the GBs matrix of $\mathrm{NMCHM}$ can also be reexpressed

$$
\Omega=e^{i \frac{|h|}{2 f} \Xi}, \quad \boldsymbol{\Sigma}(x)=e^{i \frac{|h|}{f} \Xi},
$$

where $|h|$ is the scalar singlet field and $\Xi$ is the would-be GBs nonlinear field given by

$$
\Xi=\sqrt{2} T^{\hat{a}} \hat{h}_{\hat{a}}=-i \sqrt{2} \operatorname{Tr}\left(\mathbf{U} \sigma_{\hat{\alpha}}\right) T^{\hat{\alpha}}+\sqrt{2} \hat{h}_{5} T^{\hat{5}},
$$

where $\hat{a}=1, \ldots, 5$, and we have matched it to Eq. (2.5), by using Eq. (A49) and $\sigma^{\hat{\alpha}} \equiv\left(\sigma^{1}, \sigma^{2}, \sigma^{3}, i \mathbf{1}_{2}\right)$ with $\psi$ defined in Eq. (2.17) and the definition of $\hat{h}_{a}$ in the unitary gauge as

$$
\hat{h}_{a}=0, \quad \hat{h}_{4}=c_{\psi}, \quad \hat{h}_{5}=s_{\psi} .
$$

In this case, $\Xi$ reduces to $\sqrt{2}\left(T^{\hat{4}} c_{\psi}+T^{\hat{5}} s_{\psi}\right)$.

In a general case, we can parametrize the sixdimensional fundamental scalar in $S O(6)$ as Eq. (2.4), embedding with the four-component Higgs vector in the fundamental representation of $S O(4)$ as Eq. (12) in Ref. [25] or Eq. (A.5) in Ref. [26] as 


$$
\Phi_{\hat{a}}=\left(\begin{array}{c}
s_{\phi} \hat{h}_{\hat{\alpha}} \\
s_{\phi} \hat{h}_{5} \\
c_{\phi}
\end{array}\right)=\left(\begin{array}{c}
s_{\phi} \frac{1}{2} \operatorname{Tr}\left[\mathbf{U} \sigma_{\hat{\alpha}}\right] \\
s_{\phi} \hat{h}_{5} \\
c_{\phi}
\end{array}\right),
$$

where for $h_{\hat{\alpha}}$ with $\hat{\alpha}=1,2,3,4$ and in the second equality, we have used Eq. (A48). Thus, by using the definition of Eq. (2.12), one can parametrize the $S O(6)$ fundamental scalar with the SM Higgs embedded as

$$
\Phi_{\hat{a}} \equiv\left(\begin{array}{c}
s_{\phi} c_{\psi}\left(s_{\varphi} \hat{\varphi}_{a}\right) \\
s_{\phi} c_{\psi}\left(c_{\varphi}\right) \\
s_{\phi} s_{\psi} \\
c_{\phi}
\end{array}\right) \stackrel{v \gg 1}{=}\left(\begin{array}{c}
s_{\phi} c_{\psi} \frac{\varphi_{a}}{v} \\
s_{\phi} c_{\psi} \\
s_{\phi} s_{\psi} \\
c_{\phi}
\end{array}\right) .
$$

It is convenient to reexpress the fundamental scalar in the unitary gauge

$\Phi_{\hat{a}}=\left(\begin{array}{c}0_{3} \\ c_{\psi} s_{\phi} \\ s_{\psi} s_{\phi} \\ c_{\phi}\end{array}\right)=\left(\begin{array}{c}0_{3} \\ \frac{h_{4} s_{\phi}}{\sqrt{h_{4}^{2}+h_{5}^{2}}} \\ \frac{h_{5} s_{\phi}}{\sqrt{h_{4}^{2}+h_{5}^{2}}} \\ c_{\phi}\end{array}\right)=\left(\begin{array}{c}0_{3} \\ \frac{h}{f} \\ \frac{s}{f} \\ \sqrt{1-\frac{h^{2}+s^{2}}{f^{2}}}\end{array}\right)$,

where in the second equality, we change into $(h, s)$ basis by using definitions in Eq. (2.18). This gives the square root nonlinear parametrization of $S O(6)$ in analogy to that of $S O(4)$ in Eq. (A53). In the absence of the singlet $s$, the fundamental representation of the scalar recovers that of $\mathrm{MCHM}$ in $S O(5) / S O(4)$ as $[13,17]$

$$
\Phi_{\hat{a}}=\left(\begin{array}{c}
s_{h} s_{\varphi} \hat{\varphi}_{a} \\
s_{h} c_{\varphi} \\
0 \\
c_{h}
\end{array}\right) \stackrel{\varphi \rightarrow 0}{=}\left(\begin{array}{c}
0 \\
s_{h} \\
0 \\
c_{h}
\end{array}\right) .
$$

\section{Embedding with rotation $\theta$}

The vacuum can be associated with an angle $\theta$, as the rotation angle in an $S^{5}$ unit sphere. The vacuum in the fundamental representation in Eq. (2.4), i.e., a fivedimensional unit vector $\boldsymbol{\Sigma}_{0}$, under the rotation $R(\theta)$ in Eq. (2.32), becomes

$$
\Phi_{0}(\theta)=R(\theta) \Phi_{0}=\left(\begin{array}{c}
0_{3 \times 1} \\
s_{\theta} \\
0 \\
c_{\theta}
\end{array}\right),
$$

where $0_{3 \times 1}=(0,0,0)^{T}$, and the angle $\theta$ parametrizes the misalignment of the vacuum with respect to the original vacuum.
The pseudo-Nambu goldstone boson in the fundamental representation of $S O(6)$ in Eq. (2.62) becomes

$$
\Phi_{\hat{a}}=\left(\begin{array}{c}
\left(c_{\theta} s_{\phi}-2 \hat{h}_{4} s_{\theta} s_{\frac{\phi}{2}}^{2}\right) \hat{\vec{h}}^{T} \\
\hat{h}_{4} c_{\theta} s_{\phi}-2 \hat{h}_{4}^{2} s_{\theta} s_{\frac{\phi}{2}}^{2}+s_{\theta} \\
\hat{h}_{5}\left(c_{\theta} s_{\phi}-2 \hat{h}_{4} s_{\theta} s_{\frac{\phi}{2}}^{2}\right) \\
c_{\theta} c_{\phi}-\hat{h}_{4} s_{\theta} s_{\phi}
\end{array}\right) \stackrel{\theta=2 n \pi}{=}\left(\begin{array}{c}
\hat{h}_{1} s_{\phi} \\
\hat{h}_{2} s_{\phi} \\
\hat{h}_{3} s_{\phi} \\
\hat{h}_{4} s_{\phi} \\
\hat{h}_{5} s_{\phi} \\
c_{\phi}
\end{array}\right),
$$

where $\hat{\vec{h}}=\left(\hat{h}_{1}, \hat{h}_{2}, \hat{h}_{3}\right)$ with $\hat{h}_{a} \equiv h_{a} / \phi, a=1,2,3$. The SM Higgs doublet can be defined as through the first four elements of the fundamental $S O(6)$ scalar as Eq. (A33). Having the $\hat{h}_{5}$ d.o.f. decoupled, it reduces to

$$
\Phi_{\hat{a}} \stackrel{\hat{h}_{5} \sim 0}{=}\left(\begin{array}{c}
s_{\phi} \hat{\vec{h}}^{T} \\
\hat{h}_{4} c_{\theta} s_{\phi}+c_{\phi} s_{\theta} \\
0 \\
-\hat{h}_{4} s_{\phi} s_{\theta}+c_{\phi} c_{\theta}
\end{array}\right),
$$

where $\hat{\vec{h}}=\left(\hat{h}_{1}, \hat{h}_{2}, \hat{h}_{3}\right)$. In the GB-less limit, i.e., $\varphi \rightarrow 0$ by redefining $\hat{h}_{1,2,3}=\varphi_{1,2,3} / v, \hat{h}_{4}=c_{\psi}$ and $\hat{h}_{5}=s_{\psi}$, one has

$\Phi_{\hat{a}}(\theta)=\Omega(h) \Phi_{0}(\theta) \stackrel{\theta=0}{=}\left(\begin{array}{c}\frac{\varphi_{1}}{v} c_{\psi} s_{\phi} \\ \frac{\varphi_{2}}{v} c_{\psi} s_{\phi} \\ \frac{\varphi_{3}}{v} c_{\psi} s_{\phi} \\ c_{\psi} s_{\phi} \\ s_{\psi} s_{\phi} \\ c_{\phi}\end{array}\right) \stackrel{f \rightarrow \infty}{=} \Phi_{0}(\theta)$.

When $\theta=0$, the SM electroweak group is unbroken, and is embedded into the global $S O(4)$ symmetry, and the $3+1=4$ EW GBs form a complex doublet of $S U(2)_{L}$. When $\psi=0$ with a small $\theta$, then

$$
\Phi(\theta) \stackrel{\theta \sim 0}{=}\left(\begin{array}{c}
\sin \left(\frac{h}{f}+\theta\right) \frac{\langle\varphi\rangle^{T}}{v} \\
\sin \left(\frac{h}{f}+\theta\right) \\
0 \\
\cos \left(\frac{h}{f}+\theta\right)
\end{array}\right) .
$$

When $\theta \neq 0$, the SM vector bosons gauge the $S O(6) / S O(5)$ broken generators $T^{\hat{a}}$. It triggers the spontaneous symmetry breaking, after which, the degrees of freedom of the three EW GBs are transferred to the longitudinal modes of the $W^{ \pm}$and $Z$, respectively, so that 
they obtain masses, while a fourth one is identified as the Higgs boson. Therefore, the EWSB is due to the misalignment $\theta$, which can be generated at the loop level as long as the GB 4-vector acquires a vev $\langle h\rangle \neq 0$, so that

$$
\theta \equiv \frac{\langle h\rangle}{f} .
$$

It can be checked that under the automorphism symmetry in Eq. (2.38), the vacuum is inverse to itself

$$
\begin{gathered}
\mathcal{R}(\theta) \Phi(\theta)=\left(\begin{array}{c}
\frac{2}{v} \sin \left(\frac{h}{2 f}\right) \cos \left(\frac{h}{2 f}+\theta\right)\langle\varphi\rangle^{T} \\
\sin \left(\frac{h}{f}-\theta\right) \\
0 \\
-\cos \left(\frac{h}{f}-\theta\right)
\end{array}\right) \\
\stackrel{h=0}{=}-\Phi(\theta) .
\end{gathered}
$$

Thus, given a generic $\theta$, the action of $\mathcal{R}$ is linear on the $S O(6) / S O(5)$ GBs at scale $f$, while it is nonlinear on the Higgs field $h$ as well as the $S U(2)_{L}$ GBs at scale $v$.

Thus, according to Eq. (2.69), the fundamental scalar in $S O(6)$ becomes

$$
\Phi\left(h_{\hat{a}}\right)=\left(\begin{array}{c}
\hat{\varphi}^{1} s_{\varphi} \\
\sin \left(\frac{h}{f}+\theta\right)\left(\begin{array}{c}
\hat{\varphi}^{2} s_{\varphi} \\
\hat{\varphi}^{3} s_{\varphi} \\
c_{\varphi}
\end{array}\right) \\
0 \\
\cos \left(\frac{h}{f}+\theta\right)
\end{array}\right),
$$

where $h$ parametrizes the $S O(4)$ invariant quantum fluctuation around the vacuum $\langle h\rangle=f \theta$.

Under the left-right parity symmetry in Eq. (2.45), the GB changes sign, while the Higgs is invariant,

$$
\varphi^{\hat{a}} \rightarrow-\varphi^{\hat{a}}, \quad h \rightarrow h .
$$

From the two definitions in Eqs. (2.67) and (2.72), one can identify the relations between $\hat{h}$ and $\hat{\phi}$ as

$$
\begin{aligned}
\hat{h}^{a} s_{\phi} & =\sin \left(\frac{h}{f}+\theta\right) \frac{\pi^{a}}{v}, \\
\hat{h}^{4} s_{\phi} & =c_{\theta} \sin \left(\frac{h}{f}+\theta\right) \sqrt{1-\frac{\pi^{2}}{v^{2}}}-s_{\theta} \cos \left(\frac{h}{f}+\theta\right), \\
c_{\phi} & =c_{\theta} \cos \left(\frac{h}{f}+\theta\right)+s_{\theta} \sin \left(\frac{h}{f}+\theta\right) \sqrt{1-\frac{\pi^{2}}{v^{2}}},
\end{aligned}
$$

which leads to a reparametrization between $\hat{h}_{a}$ and $\left(\hat{\phi}_{a}, \hat{h}^{4}\right)$ with $a=1,2,3$ in $S O(4)$. In the above, we rewrite the linearly realized $\varphi$ in terms of nonlinearly realized $\pi$ by using Eq. (2.56).

\section{HIGH-ENERGY EFFECTIVE CHIRAL LAGRANGIAN}

\section{A. Nonlinear chiral Lagrangian in $\Sigma$ parametrization}

\section{CP-even sector}

For the $C P$-even case, one has 13 independent operators up to $\mathcal{O}\left(p^{4}\right)$,

$$
\mathcal{L}_{\text {high }}=\mathcal{L}_{\text {high }}^{p^{2}}+\mathcal{L}_{\text {high }}^{p^{4}}
$$

where the high-energy effective chiral Lagrangian $\mathcal{L}_{\text {high }}$ denotes

$\mathcal{L}_{\text {high }}^{p^{2}}=\bar{c}_{C} \overline{\mathcal{L}}_{C}+\bar{c}_{T} \overline{\mathcal{L}}_{T}$,

$\mathcal{L}_{\text {high }}^{p^{4}}=\bar{c}_{B} \overline{\mathcal{L}}_{B}+\bar{c}_{W} \overline{\mathcal{L}}_{W}+\bar{c}_{B \boldsymbol{\Sigma}} \overline{\mathcal{L}}_{B \boldsymbol{\Sigma}}+\bar{c}_{W \Sigma} \overline{\mathcal{L}}_{W \boldsymbol{\Sigma}}+\sum_{n=1}^{8} c_{n} \overline{\mathcal{L}}_{n}$,

with [49]

$$
\begin{aligned}
\overline{\mathcal{L}}_{C} & =-\frac{f^{2}}{4} \operatorname{Tr}\left(\overline{\mathrm{V}}_{\mu} \overline{\mathrm{V}}^{\mu}\right), \\
\overline{\mathcal{L}}_{T} & =-\frac{f^{2}}{4}\left[\operatorname{Tr}\left(\overline{\mathrm{V}}_{\mu} \overline{\mathbf{T}}\right)\right]^{2}, \\
\overline{\mathcal{L}}_{B} & =-\frac{1}{4} g^{\prime 2} \operatorname{Tr}\left(\overline{\mathrm{B}}_{\mu \nu} \overline{\mathrm{B}}^{\mu \nu}\right), \\
\overline{\mathcal{L}}_{W} & =-\frac{1}{4} g^{2} \operatorname{Tr}\left(\overline{\mathrm{W}}_{\mu \nu} \overline{\mathrm{W}}^{\mu \nu}\right), \\
\overline{\mathcal{L}}_{B \boldsymbol{\Sigma}} & =g^{\prime 2} \operatorname{Tr}\left(\mathbf{\Sigma} \overline{\mathrm{B}}_{\mu \nu} \boldsymbol{\Sigma}^{-1} \overline{\mathrm{B}}^{\mu \nu}\right), \\
\overline{\mathcal{L}}_{W \Sigma} & =g^{2} \operatorname{Tr}\left(\bar{\Sigma}_{\overline{\mathrm{W}}}{ }_{\mu \nu} \boldsymbol{\Sigma}^{-1} \overline{\mathrm{W}}^{\mu \nu}\right), \\
\overline{\mathcal{L}}_{1} & =g g^{\prime} \operatorname{Tr}\left(\boldsymbol{\Sigma} \overline{\mathrm{B}}_{\mu \nu} \boldsymbol{\Sigma}^{-1} \overline{\mathrm{W}}^{\mu \nu}\right), \\
\overline{\mathcal{L}}_{2} & =i g^{\prime} \operatorname{Tr}\left(\overline{\mathrm{B}}_{\mu \nu}\left[\overline{\mathrm{V}}^{\mu}, \overline{\mathrm{V}}^{\nu}\right]\right), \\
\overline{\mathcal{L}}_{3} & \left.=i g \operatorname{Tr}\left(\overline{\mathrm{W}}_{\mu \nu} \overline{\mathrm{V}}^{\mu}, \overline{\mathrm{V}}^{\nu}\right]\right), \\
\overline{\mathcal{L}}_{4} & =\operatorname{Tr}\left(\overline{\mathrm{V}}_{\mu} \overline{\mathrm{V}}^{\mu}\right) \operatorname{Tr}\left(\overline{\mathrm{V}}_{\nu} \overline{\mathrm{V}}^{\nu}\right), \\
\overline{\mathcal{L}}_{5} & =\operatorname{Tr}\left(\overline{\mathrm{V}}_{\mu} \overline{\mathrm{V}}_{\nu}\right) \operatorname{Tr}\left(\overline{\mathrm{V}}^{\mu} \overline{\mathrm{V}}^{\nu}\right), \\
\overline{\mathcal{L}}_{6} & =\operatorname{Tr}\left(\left(\mathbf{D}_{\mu} \overline{\mathrm{V}}^{\mu}\right)^{2}\right), \\
\overline{\mathcal{L}}_{7} & =\operatorname{Tr}\left(\overline{\mathrm{V}}_{\mu} \overline{\mathrm{V}}^{\mu} \overline{\mathrm{V}}_{\nu} \overline{\mathrm{V}}^{\nu}\right), \\
\overline{\mathcal{L}}_{8} & =\operatorname{Tr}\left(\overline{\mathrm{V}}_{\mu} \overline{\mathrm{V}}_{\nu} \overline{\mathrm{V}}^{\mu} \overline{\mathrm{V}}^{\nu}\right),
\end{aligned}
$$

where $\overline{\mathrm{V}}_{\mu} \equiv\left(\mathbf{D}_{\mu} \boldsymbol{\Sigma}\right) \boldsymbol{\Sigma}^{-1}$ as defined in Eq. (B34) and $\overline{\mathbf{T}} \equiv$ $\boldsymbol{\Sigma} Q_{Y} \boldsymbol{\Sigma}^{-1}$ as defined in Eq. (B38). Since $\overline{\mathrm{V}}_{\mu}$ is the vector chiral field transforming in the adjoint of the gauge field, 
the corresponding covariant derivative in the adjoint representation is defined as

$$
\mathbf{D}_{\mu} \overline{\mathrm{V}}^{\nu}=\partial_{\mu} \overline{\mathrm{V}}^{\nu}+i g_{A}\left[\overline{\mathrm{A}}_{\mu}, \overline{\mathrm{V}}^{\nu}\right] .
$$

For the SM, the explicit expression $g_{A} \bar{A}_{\mu}=g \bar{W}_{\mu}+g^{\prime} \bar{B}_{\mu}$ is given in Eq. (B22). The gauging of the SM symmetry represents an explicit breaking of global symmetry $\mathcal{G}$

$$
\mathbf{D}_{\mu} \overline{\mathrm{V}}^{\mu}=\partial_{\mu} \overline{\mathrm{V}}^{\mu}+i g\left[\overline{\mathbf{W}}_{\mu}, \overline{\mathrm{V}}^{\mu}\right]+i g^{\prime}\left[\overline{\mathrm{B}}_{\mu}, \overline{\mathrm{V}}^{\mu}\right] .
$$

The operators listed above consist of a complete set of independent operators basis in the description of the interactions among $\mathcal{G}$ gauge bosons and the GBs associated to $\mathcal{G} / \mathcal{H}$ in the $\boldsymbol{\Sigma}$ parametrization. When fermions are introduced, all operators containing $\mathrm{D}_{\mu} \overline{\mathrm{V}}^{\mu}$ can be changed into those containing fermions via equations of motion.

\section{CP-odd sector}

For the $C P$-odd case, the EW high-energy chiral Lagrangian, up to the fourth derivatives, has six independent operators as

$$
\tilde{\mathcal{L}}_{\text {high }}=\tilde{\mathcal{L}}_{\text {high }}^{p^{2}}+\tilde{\mathcal{L}}_{\text {high }}^{p^{4}},
$$

where

$$
\begin{aligned}
\tilde{\mathcal{L}}_{\text {high }}^{p^{2}}= & \bar{c}_{\tilde{T}} \overline{\mathcal{L}}_{\tilde{T}}, \\
\tilde{\mathcal{L}}_{\text {high }}^{p^{4}}= & \bar{c}_{\tilde{W}} \overline{\mathcal{L}}_{\tilde{W}}+\bar{c}_{\tilde{B} \Sigma} \overline{\mathcal{L}}_{\tilde{B} \Sigma}+\bar{c}_{\tilde{W} \Sigma} \overline{\mathcal{L}}_{\tilde{W} \Sigma} \\
& +\bar{c}_{\tilde{1}} \overline{\mathcal{L}}_{\tilde{1}}+\bar{c}_{\tilde{2}} \overline{\mathcal{L}}_{\tilde{2}}+\bar{c}_{\tilde{3}} \overline{\mathcal{L}}_{\tilde{3}},
\end{aligned}
$$

with $c_{\tilde{n}}$ as the coefficients [52]

$$
\begin{aligned}
\overline{\mathcal{L}}_{\tilde{T}} & =i \frac{f^{2}}{4} \operatorname{Tr}\left(\overline{\mathbf{T}}^{\mu} \overline{\mathbf{V}}_{\mu}\right), \\
\overline{\mathcal{L}}_{\tilde{W}} & =-\frac{g^{2}}{4} \operatorname{Tr}\left(\tilde{\overline{\mathbf{W}}}_{\mu \nu} \overline{\mathbf{W}}^{\mu \nu}\right), \\
\overline{\mathcal{L}}_{\tilde{B} \boldsymbol{\Sigma}} & =g^{\prime 2} \operatorname{Tr}\left(\tilde{\overline{\mathbf{B}}}_{\mu \nu} \boldsymbol{\Sigma} \overline{\mathrm{B}}^{\mu \nu} \boldsymbol{\Sigma}^{-1}\right), \\
\overline{\mathcal{L}}_{\tilde{W} \boldsymbol{\Sigma}} & =g^{2} \operatorname{Tr}\left(\tilde{\overline{\mathbf{W}}}_{\mu \nu} \boldsymbol{\Sigma} \overline{\mathbf{W}}^{\mu \nu} \boldsymbol{\Sigma}^{-1}\right), \\
\overline{\mathcal{L}}_{\tilde{1}} & =g g^{\prime} \operatorname{Tr}\left(\tilde{\overline{\mathbf{W}}}_{\mu \nu} \boldsymbol{\Sigma} \overline{\mathrm{B}}^{\mu \nu} \boldsymbol{\Sigma}^{-1}\right), \\
\overline{\mathcal{L}}_{\tilde{2}} & =\epsilon_{\mu \nu \rho \sigma} \operatorname{Tr}\left(\overline{\mathbf{T}}\left[\overline{\mathbf{V}}^{\mu}, \overline{\mathbf{V}}^{\nu}\right]\right) \operatorname{Tr}\left(\overline{\mathbf{T}}\left[\overline{\mathbf{V}}^{\rho}, \overline{\mathbf{V}}^{\sigma}\right]\right), \\
\overline{\mathcal{L}}_{\tilde{\mathbf{3}}} & =\epsilon_{\mu \nu \rho \sigma} \operatorname{Tr}\left(\overline{\mathbf{V}}^{\mu} \overline{\mathbf{V}}^{\nu} \overline{\mathbf{V}}^{\rho} \overline{\mathbf{V}}^{\sigma}\right),
\end{aligned}
$$

where $\overline{\mathbf{T}}$ is defined in Eq. (B38).

By substituting the explicit expressions of the building blocks for the Sigma parametrization in Eq. (2.2), into the $C P$-even or the $C P$-odd operators in the high-energy effective Lagrangian basis $\overline{\mathcal{L}}_{\text {high }}$ of Eqs. (3.1) or (3.6), respectively, one can produce the low-energy effective chiral Lagrangian basis $\mathcal{L}_{\text {low }}$ in Eqs. (C11) or (C15) for the NMCHM with $S O(6) / S O(5)$ symmetry breaking pattern, as functions of the SM gauge bosons, the SM would-be GBs, and the $C P$-even scalar field $h$ as well as the $C P$-odd scalar singlet $s$.

\section{B. Nonlinear chiral Lagrangian in $\mathbf{\Omega}$ parametrization}

The building blocks in the $\boldsymbol{\Sigma}$ parametrization, namely, $\left(\overline{\mathrm{V}}_{\mu}, \overline{\mathrm{F}}_{\mu \nu}, \overline{\mathbf{T}}\right)$, can be expressed as

$\overline{\mathrm{V}}_{\mu}=\Omega v_{\mu} \Omega^{-1}, \quad \overline{\mathrm{F}}_{\mu \nu}=\Omega f_{\mu \nu} \Omega^{-1}, \quad \overline{\mathbf{T}}=\Omega \chi \Omega^{-1}$,

where $\left(v_{\mu}, f_{\mu \nu}, \chi\right)$ are building blocks defined in $\Omega$ parametrization, as defined in Eqs. (B40), where $\chi \equiv \Omega Q_{Y} \Omega^{-1}$ is a custodial breaking operator, in analogy to $\overline{\mathbf{T}}$ operator in Eq. (B38) in $\boldsymbol{\Sigma}$ parametrization.

Thus, the $C P$-even operators in the $\boldsymbol{\Sigma}$ parametrization can be expressed as those in the $\Omega$ parametrization, e.g.,

$$
\begin{aligned}
\overline{\mathrm{V}}_{\mu} \overline{\mathrm{V}}_{\nu} & =\Omega\left(v_{\mu} v_{\nu}\right) \Omega^{-1}, \quad \operatorname{Tr}\left(\overline{\mathrm{V}}_{\mu} \overline{\mathrm{V}}^{\mu}\right)=\operatorname{Tr}\left(v_{\mu} v^{\nu}\right), \\
\overline{\mathrm{F}}_{\mu \nu} \overline{\mathrm{F}}^{\mu \nu} & =\Omega\left(f_{\mu \nu} f^{\mu \nu}\right) \Omega^{-1}, \\
\overline{\mathrm{F}}_{\mu \nu} \boldsymbol{\Sigma} \overline{\mathrm{F}}^{\mu \nu(\mathcal{R})} \boldsymbol{\Sigma}^{-1} & =\Omega\left(f_{\mu \nu} f^{\mu \nu(R)}\right) \Omega^{-1}, \\
\overline{\mathrm{F}}_{\mu \nu}\left[\overline{\mathrm{V}}^{\mu}, \overline{\mathrm{V}}^{\nu}\right] & =\Omega\left(f_{\mu \nu}\left[v^{\mu}, v^{\nu}\right]\right) \Omega^{-1}, \\
\overline{\mathbf{T}}\left[\overline{\mathrm{V}}^{\mu}, \overline{\mathrm{V}}^{\nu}\right] & =\Omega \chi \Omega^{-1}\left[v_{\mu}, v_{\nu}\right] \Omega^{-1},
\end{aligned}
$$

where (after taking trace) the operator in the second row is a kinetic term of gauge boson $\overline{\mathrm{A}}$ of the symmetry $\mathcal{G}$. The operators in the third and fourth rows contain interactions of gauge-GBs and gauge-gauge, respectively. Similarly, for the $C P$-odd case, we have

$$
\begin{aligned}
\tilde{\overline{\mathrm{F}}}_{\mu \nu} \overline{\mathrm{F}}^{\mu \nu} & =\Omega\left(\tilde{f}_{\mu \nu} f^{\mu \nu}\right) \Omega^{-1}, \\
\tilde{\overline{\mathrm{F}}}_{\mu \nu} \Sigma \overline{\mathrm{F}}^{\mu \nu, R} \Sigma^{-1} & =\Omega\left(\tilde{f}_{\mu \nu} f^{\mu \nu(\mathcal{R})}\right) \Omega^{-1}, \\
\tilde{\overline{\mathrm{F}}}_{\mu \nu}\left[\overline{\mathrm{V}}^{\mu}, \overline{\mathrm{V}}^{\nu}\right] & =\Omega\left(\tilde{f}_{\mu \nu}\left[v^{\mu}, v^{\nu}\right]\right) \Omega^{-1},
\end{aligned}
$$

where, (after taking trace) the operator in the first line is the topological $\theta$ phase term for the unbroken gauge group $\mathcal{H}$. The operator in the third line contains interactions between gauge bosons and GBs.

The covariant adjoint derivative in Eq. (3.4) becomes

$$
\begin{aligned}
\mathbf{D}_{\mu} \overline{\mathrm{V}}^{\nu} & =\Omega\left(\nabla_{\mu} v^{\nu}+\frac{1}{2}\left[v_{\mu}, v^{\nu}\right]\right) \Omega^{-1} \\
& =2 i \Omega\left(\nabla_{\mu} d^{\nu}+i\left[d_{\mu}, d^{\nu}\right]\right) \Omega^{-1},
\end{aligned}
$$

where $\nabla_{\mu} v^{\nu}=\partial_{\mu} v^{\nu}+i\left[e_{\mu}, v^{\nu}\right]=\mathbf{D}_{\mu} v^{\nu}+i\left[\bar{e}_{\mu}, v^{\nu}\right]$ where $\mathbf{D}_{\mu} v^{\nu}=\partial_{\mu} v^{\nu}+i g_{A}\left[\overline{\mathrm{A}}_{\mu}, v^{\nu}\right]$ and $v^{\nu}=2 i d^{\nu}$. In the above derivation, we have used the commutation relations that $\left[\overline{\mathrm{A}}_{\mu}, \Omega\right]=\left[\Omega^{-1}, \overline{\mathrm{A}}_{\mu}\right]=0$ due to the property of unbroken generators in $\overline{\mathrm{A}}_{\mu}=\overline{\mathrm{A}}_{\mu}^{a} T^{a}$ as well as Eq. (B50). For gauge fields under the SM gauge group, i.e., $\overline{\mathrm{F}}=(\overline{\mathrm{W}}, \overline{\mathrm{B}})$, it can be written explicitly as 


$$
\overline{\mathrm{W}}_{\mu \nu}=\Omega w_{\mu \nu} \Omega^{-1}, \quad \overline{\mathrm{B}}_{\mu \nu}=\Omega b_{\mu \nu} \Omega^{-1},
$$

where we have used the new notations $f_{\mu \nu}^{-}=\left(w_{\mu \nu}, b_{\mu \nu}\right)$ which are defined in Eqs. (B53) and (B54).

In the end, all of the gauge fields $f_{\mu \nu},\left(w_{\mu \nu}, b_{\mu \nu}\right)$ can be decomposed in the automorphism states, by using Eq. (B55). On the other hand, we may use the new notations for $v_{\mu}=$ $2 i d_{\mu}$ as denoted in Eq. (B48), to make replacement for all of operators in the effective Lagrangians.

\section{CP-even sector}

In the $\Omega$ parametrization, the $C P$-even operators in Eq. (3.3) can be simplified as

$$
\begin{array}{rlrl}
\overline{\mathcal{L}}_{C} & =-\frac{f^{2}}{16} \operatorname{Tr}\left(v_{\mu} v^{\mu}\right), & & \overline{\mathcal{L}}_{2}=i g^{\prime} \operatorname{Tr}\left(b_{\mu \nu}\left[v^{\mu}, v^{\nu}\right]\right), \\
\overline{\mathcal{L}}_{T} & =-\frac{f^{2}}{16}\left[\operatorname{Tr}\left(v_{\mu} \chi\right)\right]^{2}, & & \overline{\mathcal{L}}_{3}=i g \operatorname{Tr}\left(w_{\mu \nu}\left[v^{\mu}, v^{\nu}\right]\right), \\
\overline{\mathcal{L}}_{B} & =-\frac{1}{4} g^{\prime 2} \operatorname{Tr}\left(b_{\mu \nu} b^{\mu \nu}\right), & & \overline{\mathcal{L}}_{4}=\operatorname{Tr}\left(v_{\mu} v^{\mu}\right) \operatorname{Tr}\left(v_{\nu} v^{\nu}\right), \\
\overline{\mathcal{L}}_{W} & =-\frac{1}{4} g^{2} \operatorname{Tr}\left(w_{\mu \nu} w^{\mu \nu}\right), & & \overline{\mathcal{L}}_{5}=\operatorname{Tr}\left(v_{\mu} v_{\nu}\right) \operatorname{Tr}\left(v^{\mu} v^{\nu}\right), \\
\overline{\mathcal{L}}_{B \Sigma} & =g^{\prime 2} \operatorname{Tr}\left(b_{\mu \nu}^{(\mathcal{R})} b^{\mu \nu}\right), & & \overline{\mathcal{L}}_{6}=\operatorname{Tr}\left(\left(\nabla_{\mu} v^{\mu}\right)^{2}\right), \\
\overline{\mathcal{L}}_{W \Sigma}=g^{2} \operatorname{Tr}\left(w_{\mu \nu}^{(\mathcal{R})} w^{\mu \nu}\right), & & \overline{\mathcal{L}}_{7}=\operatorname{Tr}\left(v_{\mu} v^{\mu} v_{\nu} v^{\nu}\right), \\
\overline{\mathcal{L}}_{1}=g g^{\prime} \operatorname{Tr}\left(b_{\mu \nu}^{(\mathcal{R})} w^{\mu \nu}\right), & & \overline{\mathcal{L}}_{8}=\operatorname{Tr}\left(v_{\mu} v_{\nu} v^{\mu} v^{\nu}\right),
\end{array}
$$

or, equivalently,

$$
\begin{aligned}
\overline{\mathcal{L}}_{C} & =\frac{f^{2}}{4} \operatorname{Tr}\left(d_{\mu} d^{\mu}\right), \\
\overline{\mathcal{L}}_{T} & =\frac{f^{2}}{4}\left[\operatorname{Tr}\left(d_{\mu} \chi\right)\right]^{2}, \\
\overline{\mathcal{L}}_{B} & =-\frac{1}{4} g^{\prime 2} \operatorname{Tr}\left[\left(b_{\mu \nu}^{+}+b_{\mu \nu}^{-}\right)^{2}\right], \\
\overline{\mathcal{L}}_{W} & =-\frac{1}{4} g^{2} \operatorname{Tr}\left[\left(w_{\mu \nu}^{+}+w_{\mu \nu}^{-}\right)^{2}\right], \\
\overline{\mathcal{L}}_{B \Sigma} & =g^{\prime 2} \operatorname{Tr}\left[\left(b_{\mu \nu}^{+}\right)^{2}-\left(b_{\mu \nu}^{-}\right)^{2}\right], \\
\overline{\mathcal{L}}_{W \Sigma} & =g^{2} \operatorname{Tr}\left[\left(w_{\mu \nu}^{+}\right)^{2}-\left(w_{\mu \nu}^{-}\right)^{2}\right], \\
\overline{\mathcal{L}}_{1} & =g g^{\prime} \operatorname{Tr}\left[\left(b_{\mu \nu}^{+}-b_{\mu \nu}^{-}\right) w^{\mu \nu}\right], \\
\overline{\mathcal{L}}_{2} & =-4 i g^{\prime} \operatorname{Tr}\left(\left(b_{\mu \nu}^{+}+b_{\mu \nu}^{-}\right)\left[d^{\mu}, d^{\nu}\right]\right), \\
\overline{\mathcal{L}}_{3} & =-4 i g \operatorname{Tr}\left(\left(w_{\mu \nu}^{+}+w_{\mu \nu}^{-}\right)\left[d^{\mu}, d^{\nu}\right]\right), \\
\overline{\mathcal{L}}_{4} & =16 \operatorname{Tr}\left(d_{\mu} d^{\mu}\right) \operatorname{Tr}\left(d_{\nu} d^{\nu}\right), \\
\overline{\mathcal{L}}_{5} & =16 \operatorname{Tr}\left(d_{\mu} d_{\nu}\right) \operatorname{Tr}\left(d^{\mu} d^{\nu}\right), \\
\overline{\mathcal{L}}_{6} & =-4 \operatorname{Tr}\left[\left(\nabla_{\mu} d^{\mu}\right)^{2}\right], \\
\overline{\mathcal{L}}_{7} & =16 \operatorname{Tr}\left(d_{\mu} d^{\mu} d_{\nu} d^{\nu}\right), \\
\overline{\mathcal{L}}_{8} & =16 \operatorname{Tr}\left(d_{\mu} d_{\nu} d^{\mu} d^{\nu}\right),
\end{aligned}
$$

where there is an addition factor $1 / 4$ in front of $\overline{\mathcal{L}}_{C}$ by imposing the rule in Eq. (2.25), since we are going from $\boldsymbol{\Sigma}$ to $\Omega$ parametrization. $w_{\mu \nu}^{ \pm}, b_{\mu \nu}^{ \pm}$can be expressed in terms of CCWZ covariant building blocks $d_{\mu}, e_{\mu \nu}$ as explicitly shown in Eq. (B66).

\section{CP-odd sector}

In the $\Omega$ parametrization, the $C P$-odd operators in Eq. (3.8) can be simplified as

$$
\begin{aligned}
\overline{\mathcal{L}}_{\tilde{T}} & =i \frac{f^{2}}{16} \operatorname{Tr}\left[\left(\nabla_{\mu} v^{\mu}\right) \chi\right], \\
\overline{\mathcal{L}}_{\tilde{W}} & =-\frac{1}{4} g^{2} \operatorname{Tr}\left(\tilde{w}_{\mu \nu} w^{\mu \nu}\right), \\
\overline{\mathcal{L}}_{\tilde{\mathbf{B}} \boldsymbol{\Sigma}} & =g^{\prime 2} \operatorname{Tr}\left(\tilde{b}_{\mu \nu} b^{(\mathcal{R}) \mu \nu}\right), \\
\overline{\mathcal{L}}_{\tilde{W} \Sigma} & =g^{2} \operatorname{Tr}\left(\tilde{w}_{\mu \nu} w^{(\mathcal{R}) \mu \nu}\right), \\
\overline{\mathcal{L}}_{\tilde{1}} & =g g^{\prime} \operatorname{Tr}\left(\tilde{w}_{\mu \nu} b^{\mathcal{R} \mu \nu}\right), \\
\overline{\mathcal{L}}_{\tilde{2}} & =\epsilon_{\mu \nu \rho \sigma} \operatorname{Tr}\left(\chi\left[v^{\mu}, v^{\nu}\right]\right) \operatorname{Tr}\left(\chi\left[v^{\rho}, v^{\sigma}\right]\right), \\
\overline{\mathcal{L}}_{\tilde{3}} & =\epsilon_{\mu \nu \rho \sigma} \operatorname{Tr}\left(v^{\mu} v^{\nu} v^{\rho} v^{\sigma}\right),
\end{aligned}
$$

or equivalently,

$$
\begin{aligned}
\overline{\mathcal{L}}_{\tilde{T}} & =-\frac{f^{2}}{8} \operatorname{Tr}\left[\left(\nabla_{\mu} d^{\mu}\right) \chi\right], \\
\overline{\mathcal{L}}_{\tilde{W}} & =-\frac{1}{4} g^{2} \operatorname{Tr}\left[\left(\tilde{w}_{\mu \nu}^{+}+\tilde{w}_{\mu \nu}^{-}\right)\left(w^{+\mu \nu}+w^{-\mu \nu}\right)\right], \\
\overline{\mathcal{L}}_{\tilde{B} \boldsymbol{\Sigma}} & =g^{\prime 2} \operatorname{Tr}\left[\left(\tilde{b}_{\mu \nu}^{+}+\tilde{b}_{\mu \nu}^{-}\right)\left(b^{+\mu \nu}-b^{-\mu \nu}\right)\right], \\
\overline{\mathcal{L}}_{\tilde{W} \Sigma} & =g^{2} \operatorname{Tr}\left[\left(\tilde{w}_{\mu \nu}^{+}+\tilde{w}_{\mu \nu}^{-}\right)\left(w^{+\mu \nu}-w^{-\mu \nu}\right)\right], \\
\overline{\mathcal{L}}_{\tilde{1}} & =-g g^{\prime} \operatorname{Tr}\left[\left(\tilde{w}_{\mu \nu}^{+}+\tilde{w}_{\mu \nu}^{-}\right)\left(b^{+\mu \nu}-b^{-\mu \nu}\right)\right], \\
\overline{\mathcal{L}}_{\tilde{2}} & =16 \epsilon_{\mu \nu \rho \sigma} \operatorname{Tr}\left(\chi\left[d^{\mu}, d^{\nu}\right]\right) \operatorname{Tr}\left(\chi\left[d^{\rho}, d^{\sigma}\right]\right), \\
\overline{\mathcal{L}}_{\tilde{3}} & =16 \epsilon_{\mu \nu \rho \sigma} \operatorname{Tr}\left(d^{\mu} d^{\nu} d^{\rho} d^{\sigma}\right) .
\end{aligned}
$$

Therefore, the building blocks for the Omega parametrization in the CCWZ formalism are all

$$
d_{\mu}, \quad e_{\mu \nu}, \quad f_{\mu \nu} .
$$

Since $f_{\mu \nu}^{ \pm}=\left(w_{\mu \nu}^{ \pm}, b_{\mu \nu}^{ \pm}\right)$are not independent of $d_{\mu}$ and $e_{\mu \nu}$, one can make a choice as Eq. (B77).

With the building blocks shown above, we can obtain the high-energy effective Lagrangian up to $p^{4}$ order.

In the CCWZ approach, according to Eq. (B26), by projecting upon the broken generators of the Maurer-Cartan form, one obtains the elements of $d_{\mu}$ and $e_{\mu}$ fields, along broken and unbroken generators, respectively as 


$$
\begin{aligned}
d_{\mu}^{\hat{a}} & =\operatorname{Tr}\left(\omega_{\mu} T^{\hat{a}}\right)=-i \operatorname{Tr}\left[\left(\Omega^{\dagger} \partial_{\mu} \Omega\right) T^{\hat{a}}\right], \\
e_{\mu}^{a}=\operatorname{Tr}\left(\omega_{\mu} T^{a}\right) & =-i \operatorname{Tr}\left[\left(\Omega^{\dagger} \partial_{\mu} \Omega\right) T^{a}\right],
\end{aligned}
$$

where $\Omega$ is defined in Eq. (2.9), $\Omega^{\dagger}=\Omega^{-1}$.

In the NMCHM, the broken generators $T^{\hat{a}}$ are given in Eqs. (A5) and (A7), and can be expressed as

$$
T^{\hat{a}}=-i \frac{1}{\sqrt{2}}\left(\begin{array}{c|c}
\mathbf{0}_{4} & 1_{\hat{a}} \\
\hline-1_{\hat{a}}^{T} & 0
\end{array}\right),
$$

where $\mathbf{0}_{4}=\operatorname{diag}(0,0,0,0), 1_{\hat{a}}$ is a 4 -vector with only nonvanishing component 1 along the $\hat{a}$ th direction. The factor in front is normalization of the generators in Eq. (A1).

The $\omega_{\mu} \equiv-i \Omega^{-1} \partial_{\mu} \Omega$ is defined as introduced in Eq. (B26) in the vector formalism as

$$
\omega_{\mu}=-i\left(\begin{array}{l|l}
m_{11} & m_{12} \\
\hline m_{21} & m_{22}
\end{array}\right),
$$

with the block components as

$$
\begin{aligned}
m_{11}= & -\frac{1}{2} s_{\phi}^{2}\left[\left(\partial_{\mu} \hat{\phi}\right) \hat{\phi}^{T}-\hat{\phi}\left(\partial_{\mu} \hat{\phi}^{T}\right)\right], \quad m_{22}=s_{\phi}\left(\partial_{\mu} \hat{\phi}\right) \\
m_{12}= & \frac{1}{f}\left(\partial_{\mu}|\phi|\right) \hat{\phi}+s_{\phi}\left(\partial_{\mu} \hat{\phi}\right)-\left(1-c_{\phi}\right) s_{\phi} \hat{\phi} \hat{\phi}^{T}\left(\partial_{\mu} \hat{\phi}\right) \\
m_{21}= & -\frac{1}{f}\left(\partial_{\mu}|\phi|\right) \hat{\phi}^{T}+s_{\phi}\left(1-c_{\phi}\right)\left(\partial_{\mu} \hat{\phi}^{T}\right)\left(\hat{\phi} \hat{\phi}^{T}\right) \\
& -s_{\phi}\left(\partial_{\mu} \hat{\phi}^{T}\right)
\end{aligned}
$$

where $\hat{\phi} \hat{\phi}^{T}=\mathbf{1}$ so that $\hat{\phi} \hat{\phi}^{T}=\left(\hat{\phi} \hat{\phi}^{T}\right)^{2}$ and $\partial_{\mu}\left(\hat{\phi}^{T} \hat{\phi}\right)=0$ are used.

With the explicit expression of $\omega_{\mu}$ given in Eq. (3.22), one can read

$$
d_{\mu}^{\hat{a}}=\sqrt{2}\left(\frac{1}{f}\left(\partial_{\mu}|\phi|\right) \hat{\phi}^{\hat{a}}+s_{\phi} c_{\phi}\left(\partial_{\mu} \hat{\phi}^{\hat{a}}\right)\right) .
$$

By using the identities

$$
\begin{aligned}
\partial_{\mu}\left(\hat{\phi} \hat{\phi}^{T}\right) & =\frac{\partial_{\mu}\left(\phi \phi^{T}\right)}{|\phi|^{2}}-2 \frac{\partial_{\mu}|\phi|}{|\phi|^{3}}\left(\phi \phi^{T}\right), \\
\partial_{\mu} \hat{\phi} & =\frac{1}{|\phi|} \partial_{\mu} \phi-\frac{\partial_{\mu}|\phi|}{|\phi|^{2}} \phi
\end{aligned}
$$

$d_{\mu}$ can be reexpressed as

$d_{\mu}^{\hat{a}}=\sqrt{2}\left[\frac{1}{2}\left(\frac{2 s_{\phi} c_{\phi}}{|\phi|}-\frac{1}{f}\right) \frac{\phi^{\hat{a}}}{|\phi|^{2}} \partial_{\mu}|\phi|^{2}-\frac{2 s_{\phi} c_{\phi}}{|\phi|} \partial_{\mu} \phi^{\hat{a}}\right]$.
The kinetic derivative $\partial_{\mu}$ above can be generalized to be the covariant derivative as that in Eq. (B20)

$$
\partial_{\mu} \rightarrow D_{\mu}=\partial_{\mu}+i\left[A_{\mu}^{a} T^{a}, \mathbf{\Sigma}\right]+i\left\{A_{\mu}^{\hat{a}} T^{\hat{a}}, \mathbf{\Sigma}\right\} .
$$

For the SM, $\left\{W_{\mu}^{a}, B_{\mu}\right\} \in A_{\mu}^{a}$, we have

$$
D_{\mu}=\partial_{\mu}+i\left[W_{\mu}^{a} Q_{L}^{a}, \boldsymbol{\Sigma}\right]+i\left[B_{\mu} Q_{Y}, \mathbf{\Sigma}\right],
$$

where the gauge couplings $g, g^{\prime}$ are absorbed into the $W_{\mu}^{a}$ and $B_{\mu}$, respectively. The explicit expression of the covariant derivative terms as well as the Omega parametrization building blocks in the EW sector of NMCHM are shown in Appendix $1 \mathrm{~d}$.

\section{MATCHING TO EW CHIRAL LAGRANGIAN}

\section{A. $\boldsymbol{C P \text { -even sector }}$}

\section{1. $p^{2}$ order in $\Sigma$ parametrization}

The leading-order high-energy effective Lagrangian up to the two derivatives is

$$
\begin{aligned}
\overline{\mathcal{L}}_{C}= & \frac{1}{2}\left(\partial_{\mu} \phi\right)^{2}+2 \sin ^{2}\left(\frac{\phi}{2 f}\right)\left(\partial_{\mu} \psi\right)^{2} \\
& -f^{2} \cos ^{2}\left(\frac{\psi}{f}\right) \sin ^{2}\left(\frac{\phi}{2 f}\right) \operatorname{Tr}\left(\mathbf{V}^{\mu} \mathbf{V}_{\mu}\right) \\
\equiv & \mathcal{F}_{H} \mathcal{L}_{H}+\mathcal{F}_{s} \mathcal{L}_{s}+\mathcal{F}_{C} \mathcal{L}_{C} \\
\overline{\mathcal{L}}_{T}= & -\frac{1}{\xi} \cos ^{4}\left(\frac{\psi}{f}\right) \sin ^{4}\left(\frac{\phi}{2 f}\right) \mathcal{L}_{T} \\
\equiv & \mathcal{F}_{T} \mathcal{L}_{T},
\end{aligned}
$$

where we have used Eq. (B34) in the $\boldsymbol{\Sigma}$ parametrization. The first lines are kinetic terms for the field $\phi$, in analogy to $\mathcal{L}_{H}$ defined in Eq. (C9). The leading-order operator $\mathcal{L}_{C, T}$ is associated with the two gauge bosons as defined in Eq. (C10) or more explicitly shown in Eq. (C14) in Appendix. C.

In the absence of the singlet, i.e., $\psi=0$ (or $s=0$ ), the leading-order high-energy effective Lagrangian in the NMCHM just leads to those in the MCHM as $[13,17]$

$$
\begin{aligned}
& \overline{\mathcal{L}}_{C}=\frac{1}{2}\left(\partial_{\mu} h\right)^{2}-f^{2} \sin ^{2}\left(\frac{h}{2 f}\right) \operatorname{Tr}\left(\mathbf{V}^{\mu} \mathbf{V}_{\mu}\right), \\
& \overline{\mathcal{L}}_{T}=-\frac{1}{\xi} \sin ^{4}\left(\frac{h}{2 f}\right) \mathcal{L}_{T},
\end{aligned}
$$

where the first two terms describe the low-energy projection of the custodial preserving two derivative operators.

\section{2. $p^{4}$ order in $\Sigma$ parametrization}

In the NMCHM, the operators in the high-energy effective chiral Lagrangian in Eq. (3.3) are related to the low-energy chiral EW Lagrangian in Eq. (C11) 


$$
\begin{aligned}
& \overline{\mathcal{L}}_{B}=\mathcal{L}_{B}, \\
& \overline{\mathcal{L}}_{W}=\mathcal{L}_{W}, \\
& \overline{\mathcal{L}}_{B \Sigma}=-4\left[1-\cos ^{2}\left(\frac{\psi}{f}\right) \sin ^{2}\left(\frac{\phi}{2 f}\right)\right] \mathcal{L}_{B}, \\
& \overline{\mathcal{L}}_{W \Sigma}=-4\left[1-\cos ^{2}\left(\frac{\psi}{f}\right) \sin ^{2}\left(\frac{\phi}{2 f}\right)\right] \mathcal{L}_{W}, \\
& \overline{\mathcal{L}}_{1}=\cos ^{2}\left(\frac{\psi}{f}\right) \sin ^{2}\left(\frac{\phi}{2 f}\right) \mathcal{L}_{1}, \\
& \overline{\mathcal{L}}_{2}=\cos ^{2}\left(\frac{\psi}{f}\right) \sin ^{2}\left(\frac{\phi}{2 f}\right) \mathcal{L}_{2}+\cos ^{2}\left(\frac{\psi}{f}\right) \sin \left(\frac{\phi}{f}\right) \mathcal{L}_{4}^{(\phi)} \\
& -2 \sin \left(\frac{2 \psi}{f}\right) \sin ^{2}\left(\frac{\phi}{2 f}\right) \mathcal{L}_{4}^{(\psi)}, \\
& \overline{\mathcal{L}}_{3}=2 \cos ^{2}\left(\frac{\psi}{f}\right) \sin ^{2}\left(\frac{\phi}{2 f}\right) \mathcal{L}_{3}-2 \cos ^{2}\left(\frac{\psi}{f}\right) \sin \left(\frac{\phi}{f}\right) \mathcal{L}_{5}^{(\phi)} \\
& +4 \sin \left(\frac{2 \psi}{f}\right) \sin ^{2}\left(\frac{\phi}{2 f}\right) \mathcal{L}_{5}^{(\psi)}, \\
& \overline{\mathcal{L}}_{4}=4 \mathcal{L}_{D \phi}+16 \cos ^{4}\left(\frac{\psi}{f}\right) \sin ^{4}\left(\frac{\phi}{2 f}\right) \mathcal{L}_{6} \\
& -16 \cos ^{2}\left(\frac{\psi}{f}\right) \sin ^{2}\left(\frac{\phi}{2 f}\right) \mathcal{L}_{20}^{(\phi)}+64 \sin ^{4}\left(\frac{\phi}{2 f}\right) \mathcal{L}_{D \psi} \\
& -64 \cos ^{2}\left(\frac{\psi}{f}\right) \sin ^{4}\left(\frac{\phi}{2 f}\right) \mathcal{L}_{20}^{(\psi)} \\
& \overline{\mathcal{L}}_{5}=4 \mathcal{L}_{D \phi}+16 \cos ^{4}\left(\frac{\psi}{f}\right) \sin ^{4}\left(\frac{\phi}{2 f}\right) \mathcal{L}_{11} \\
& -16 \cos ^{2}\left(\frac{\psi}{f}\right) \sin ^{2}\left(\frac{\phi}{2 f}\right) \mathcal{L}_{8}^{(\phi)}+64 \sin ^{4}\left(\frac{\phi}{2 f}\right) \mathcal{L}_{D \psi} \\
& -64 \cos ^{2}\left(\frac{\psi}{f}\right) \sin ^{4}\left(\frac{\phi}{2 f}\right) \mathcal{L}_{8}^{(\psi)}, \\
& \overline{\mathcal{L}}_{6}-2 \cos ^{2}\left(\frac{\psi}{f}\right) \sin ^{2}\left(\frac{\phi}{2 f}\right)\left[1-\cos ^{2}\left(\frac{\psi}{f}\right) \sin ^{2}\left(\frac{\phi}{2 f}\right)\right] \mathcal{L}_{6} \\
& +4 \cos ^{2}\left(\frac{\psi}{f}\right) \sin ^{2}\left(\frac{\phi}{2 f}\right) \mathcal{L}_{9} \\
& -2 \mathcal{L}_{\square \phi}+4 \cos ^{2}\left(\frac{\psi}{f}\right) \cos ^{2}\left(\frac{\phi}{2 f}\right) \mathcal{L}_{8}^{(\phi)} \\
& -8 \sin ^{2}\left(\frac{\phi}{2 f}\right) \mathcal{L}_{\square \psi}+16 \sin ^{2}\left(\frac{\psi}{f}\right) \sin ^{2}\left(\frac{\phi}{2 f}\right) \mathcal{L}_{8}^{(\psi)} \\
& -2 \cos ^{2}\left(\frac{\psi}{f}\right) \sin \left(\frac{\phi}{f}\right)\left(\mathcal{L}_{7}^{(\phi)}-2 \mathcal{L}_{10}^{(\phi)}\right) \\
& +4 \sin \left(\frac{2 \psi}{f}\right) \sin ^{2}\left(\frac{\phi}{2 f}\right)\left(\mathcal{L}_{7}^{(\psi)}-2 \mathcal{L}_{10}^{(\psi)}\right), \\
& \overline{\mathcal{L}}_{7}=\frac{1}{4}\left(\overline{\mathcal{L}}_{4}+\overline{\mathcal{L}}_{5}\right), \\
& \overline{\mathcal{L}}_{8}=\frac{1}{2} \overline{\mathcal{L}}_{5} \text {, }
\end{aligned}
$$

where $\mathcal{L}_{n}^{(\phi)}, \mathcal{L}_{n}^{(\psi)}$ are defined as $\mathcal{L}_{n}$ with the following replacement rules:

$$
h / v \rightarrow \phi / f, \quad \text { or, } \quad h / v \rightarrow \psi / f,
$$

respectively, and similarly for $\mathcal{L}_{D \phi}$ and $\mathcal{L}_{\square \phi}$ etc.

The $\overline{\mathcal{L}}_{7,8}$ do not give independent contributions since they can be expressed in linear combinations of other operators. The traces of four $\overline{\mathrm{V}}_{\mu}$ can be expressed as products of traces of two $\overline{\mathrm{V}}_{\mu}$. Therefore, there are $2+6=8$ independent operators. The explicit expression of the low-energy chiral Lagrangian in NMCHM are list in Eq. (C14) in Appendix. C. in the absence of the $C P$-odd singlet, $\psi=0\left(s=0\right.$, or $\left.h_{5}=0\right)$, the result just recovers Eq. (4.18) in Ref. [49] in the MCHM.

The above are model-dependent relations relating the d.o.f. of the high-energy sector to that of the low-energy sector. These are still valid even after EWSB in which Higgs obtains vev at EW scale $v$ as will be discussed in Eq. (1.7).

\section{B. $C P$-odd sector}

\section{1. $p^{2}$ order: Topological $\theta$ phase}

The leading-order operator with at most two derivative in terms of $p^{2}$ order reads as

$$
\overline{\mathcal{L}}_{\tilde{T}}=-\frac{1}{\xi} \cos ^{2}\left(\frac{\psi}{f}\right) \sin ^{2}\left(\frac{\phi}{2 f}\right) \mathcal{L}_{\tilde{T}}
$$

\section{2. $p^{4}$ order: Higgs couplings to gauge bosons}

For the NMCHM, the dimension four operators in the high-energy effective Lagrangian in Eq. (3.8) are related to the low-energy EW chiral Lagrangian in Eq. (C15) as

$$
\begin{aligned}
\overline{\mathcal{L}}_{\tilde{B}}= & 0, \\
\overline{\mathcal{L}}_{\tilde{W}}= & \mathcal{L}_{\tilde{W}} \\
\overline{\mathcal{L}}_{\tilde{B} \Sigma}= & -4\left[1-\cos ^{2}\left(\frac{\psi}{f}\right) \sin ^{2}\left(\frac{\phi}{2 f}\right)\right] \mathcal{L}_{\tilde{B}} \\
\overline{\mathcal{L}}_{\tilde{W} \Sigma}= & -4\left[1-\cos ^{2}\left(\frac{\psi}{f}\right) \sin ^{2}\left(\frac{\phi}{2 f}\right)\right] \mathcal{L}_{\tilde{W}}, \\
\overline{\mathcal{L}}_{\tilde{1}}= & \frac{1}{2} \cos ^{2}\left(\frac{\psi}{f}\right) \sin ^{2}\left(\frac{\phi}{2 f}\right) \mathcal{L}_{\tilde{1}} \\
\overline{\mathcal{L}}_{\tilde{2}}= & 4 \cos ^{4}\left(\frac{\psi}{f}\right) \sin ^{4}\left(\frac{\phi}{2 f}\right)\left(\mathcal{L}_{\tilde{B}}-\mathcal{L}_{\tilde{W}}\right) \\
& +2 \cos ^{4}\left(\frac{\psi}{f}\right) \cos \left(\frac{\phi}{2 f}\right) \sin ^{3}\left(\frac{\phi}{2 f}\right)\left(\mathcal{L}_{\tilde{2}}^{(\phi)}+2 \mathcal{L}_{\tilde{3}}^{(\phi)}\right), \\
\overline{\mathcal{L}}_{\tilde{3}}= & 0,
\end{aligned}
$$

where we list the explicit expressions of $C P$-odd lowenergy chiral effective Lagrangian in Eqs. 482. The first 
two are topological $\theta$ phase terms for non-Abelian gauge symmetry and analogous to the QCD $\theta$ term. Namely, in principle, one has the EW $\theta$ term as a free parameter in the SM. For $\mathcal{L}_{\tilde{2}, \tilde{3}}^{(\phi)}$, we also used the rules setup in Eq. (4.4). In the last equality, we have used that $\overline{\mathcal{L}}_{\tilde{3}}=$ $\epsilon^{\mu \nu \rho \sigma} \operatorname{Tr}\left(\overline{\mathrm{V}}_{\mu} \overline{\mathrm{V}}_{\nu}\right) \operatorname{Tr}\left(\overline{\mathrm{V}}_{\rho} \overline{\mathrm{V}}_{\sigma}\right)$ for $S O(6) / S O(5)$. It is interesting to observe that, at $p^{4}$ order, which can be due to contributions from one loops, the low-energy chiral Lagrangian of the $\overline{\mathcal{L}}_{\tilde{B}, \tilde{W} \Sigma}$ would lead to a nonvanishing topological $\theta$ phase. In the absence of the $C P$-odd singlet, i.e, $\psi=0$ or $s=0$, the results just recover Eq. (4.6) in Ref. [52] for MCHM. We also make a comparison with a set of high-energy effective operators defined in literature as explicitly calculated in Appendix. B 7.

\section{HIGGS FUNCTIONS}

In this section, we express the Higgs functions in the low-energy chiral Lagrangian in terms of the Higgs dependence on a high-energy Lagrangian.

The low-energy EW chiral Lagrangian that describes the gauge-Goldstone and the gauge-scalar interactions up to the four derivatives can be written as

$$
\mathcal{L}_{\text {low }}=\mathcal{L}_{\text {low }}^{p^{2}}+\mathcal{L}_{\text {low }}^{p^{4}}
$$

which is explicitly described in Appendix C. To match the Wilson coefficients of the low-energy effective operators to the high-energy Higgs-dependent function, we need to match the different degrees of freedom above and below the EW scale.

\section{A. Higgs kinetic terms below EW scale}

\section{The $(\phi, \psi)$ basis: Polar coordinates}

The $C P$-even $p^{2}$ order high-energy effective Lagrangian with at most two derivatives is a custodial preserving one as given in Eq. (4.1) for NMCHM, in the $\Omega$ parametrization [26]

$$
\begin{aligned}
\overline{\mathcal{L}}_{C}= & \mathcal{L}_{\phi}+\sin ^{2}\left(\frac{\phi}{f}\right) \mathcal{L}_{\psi} \\
& -\frac{f^{2}}{4} \cos ^{2}\left(\frac{\psi}{f}\right) \sin ^{2}\left(\frac{\phi}{f}\right) \operatorname{Tr}\left(\mathbf{V}^{\mu} \mathbf{V}_{\mu}\right), \\
\overline{\mathcal{L}}_{T}= & -\frac{1}{4 \xi} \cos ^{4}\left(\frac{\psi}{f}\right) \sin ^{4}\left(\frac{\phi}{f}\right) \mathcal{L}_{T},
\end{aligned}
$$

where $\mathcal{L}_{C}$ is the custodial preserving two derivative operators as defined in Eqs. (C10) with explicit expression given in Eq. (C14). The first line describes the kinetic terms and the second line describes the scalar gauge boson couplings. $\mathcal{L}_{\phi, \psi}$ is defined in analogy to $\mathcal{L}_{H}$ in Eq. (C9), which are canonically normalized kinetic terms for the field $\phi$ and the phase $\psi$. To be explicit, the kinetic term is

$$
\mathcal{L}_{\text {kin }}=\frac{1}{2}\left(\partial_{\mu} \phi\right)\left(\partial^{\mu} \phi\right)+\frac{1}{2} \sin ^{2}\left(\frac{\phi}{f}\right)\left(\partial_{\mu} \psi\right)\left(\partial^{\mu} \psi\right),
$$

which just recovers Eq. (5.18) in the Cartesian $(h, s)$ basis. This result is consistent with that in Eq. (A.34) for MCHM in Ref. [49].

After EWSB, the scalars obtain vevs

$$
\phi \rightarrow \hat{\phi}+\langle\phi\rangle, \quad \psi \rightarrow \hat{\psi}+\langle\psi\rangle
$$

The SM gauge bosons $W$ and $Z$, obtain masses from the last term in Eq. (5.2),

$$
\begin{aligned}
\mathcal{L}_{\text {mass }} & =-\frac{f^{2}}{4} \cos ^{2}\left(\frac{v_{\psi}}{f}\right) \sin ^{2}\left(\frac{v_{\phi}}{f}\right) \operatorname{Tr}\left(\mathbf{V}^{\mu} \mathbf{V}_{\mu}\right) \\
& \equiv \frac{1}{2} m_{W}^{2}\left[\left(\left(W_{\mu}^{1}\right)^{2}+\left(W_{\mu}^{2}\right)^{2}\right)+\cdots\right],
\end{aligned}
$$

where the $W$ gauge boson mass by definition should be consistent with the definition of the EW scale $v$. To be consistent with the definition of the EW scale $v$, defined by the $W$ mass $m_{W}^{2} \equiv g^{2} v^{2} / 4$, it is entailed to impose that

$$
v^{2} \equiv \sin ^{2}\left(\frac{\langle\phi\rangle}{f}\right) \cos ^{2}\left(\frac{\langle\psi\rangle}{f}\right),
$$

which just reproduces Eq. (1.7) with $v_{\phi} \equiv\langle\phi\rangle$ and $v_{\psi} \equiv\langle\psi\rangle$. It is convenient to define the ratio between the EW scale and the symmetry breaking scale $f$ :

$$
\xi \equiv \frac{\langle h\rangle^{2}}{f^{2}}=\sin ^{2}\left(\frac{\langle\phi\rangle}{f}\right) \cos ^{2}\left(\frac{\langle\psi\rangle}{f}\right)
$$

where $\xi$ is the parameter quantifying the d.o.f. of the nonlinearity of the Higgs dynamics as defined in (1.7).

Equivalently, from linear representation of the fundamental scalar of $S O(6)$ in Eq. (2.63), one can also write down the kinetic terms of both a scalar $\phi$ and a pseudoscalar $\psi$, which corresponds to a sphere with standard metric as [25]

$$
\mathcal{L}_{\text {kin }}=\frac{1}{2} \partial_{\mu} \Phi^{T} \partial^{\mu} \Phi=\frac{1}{2 f^{2}}\left[\left(\partial_{\mu} \phi\right)^{2}+s_{\phi}^{2}\left(\partial_{\mu} \psi\right)^{2}\right]
$$

which is consistent with the leading $p^{2}$ order of the nonlinearly realized effective chiral Lagrangian from the NMCHM, as shown in Eq. (5.2).

\section{The $(h, s)$ basis}

In the projection of the $(h, s)$ parametrization, the kinetic terms in Eq. (5.8) become 


$$
\begin{aligned}
\mathcal{L}_{\text {kin }}= & \frac{1}{2 f^{2}}\left(\left(\partial_{\mu} h\right)^{2}+\left(\partial_{\mu} s\right)^{2}+\frac{\left(h \partial_{\mu} h+s \partial_{\mu} s\right)^{2}}{f^{2}-h^{2}-s^{2}}\right) \\
& +\frac{1}{8} h^{2}\left[g^{2}\left(\left(W_{\mu}^{1}\right)^{2}+\left(W_{\mu}^{2}\right)^{2}\right)+\left(g W_{\mu}^{3}-g^{\prime} B_{\mu}\right)^{2}\right] .
\end{aligned}
$$

By making a rescaling of $(h, s) \rightarrow(f h, f s)$, so that the dimensionful $(h, s)$ becomes dimensionless, the Lagrangian $\mathcal{L}_{\text {kin }}$ reads as

$$
\begin{aligned}
\mathcal{L}_{\text {kin }}= & \frac{1}{2}\left(\left(\partial_{\mu} h\right)^{2}+\left(\partial_{\mu} s\right)^{2}+\frac{\left(h \partial_{\mu} h+s \partial_{\mu} s\right)^{2}}{1-h^{2}-s^{2}}\right) \\
& +\frac{1}{4} g^{2} f^{2} h^{2}\left(W^{+\mu} W_{\mu}^{-}+\frac{1}{2 c_{W}^{2}} Z^{\mu} Z_{\mu}\right),
\end{aligned}
$$

where $c_{W} \equiv \cos \theta_{W}=g / \sqrt{g^{2}+g^{\prime 2}}$ is by definition of the Weinberg angle $\theta_{W}$. This just recovers Eq. (11) in Ref. [23] or Eq. (A.5) in Ref. [24] for the NMCHM.

In the absence of $s$, this leads to the kinetic term of the Higgs singlet as well as the gauge boson mass term as

$\mathcal{L}_{h}=\frac{1}{2}\left[\partial_{\mu} h \partial^{\mu} h+\left(\frac{h}{v}+1\right)^{2}\left(2 m_{W}^{2} W_{\mu}^{+} W^{\mu-}+m_{Z}^{2} Z_{\mu} Z^{\mu}\right)\right]$,

where the charged weak gauge bosons are defined as $W_{\mu}^{ \pm} \equiv\left(W_{\mu}^{1} \mp i W_{\mu}^{2}\right) / \sqrt{2}$, the neutral weak gauge boson is $Z_{\mu}=g W_{\mu}^{3}-g^{\prime} B_{\mu}$ and after EWSB, i.e., $h \rightarrow \hat{h}+\langle h\rangle$, the bosons $W_{\mu}^{ \pm}$and $Z_{\mu}$ obtains mass

$$
m_{W}^{2}=\frac{1}{4} g^{2} v^{2}, \quad m_{Z}^{2}=\frac{1}{4}\left(g^{2}+g^{2}\right) v^{2} .
$$

We have the parameter $\xi \equiv v^{2} / f^{2}=v_{h}^{2} / f^{2}$ with $v_{h} \equiv\langle h\rangle$.

In the presence of singlet $s$, i.e., $s \rightarrow \hat{s}+\langle s\rangle$, the vevs $\left(v_{\phi}, v_{\psi}\right)$ can be expressed in terms of $\left(v_{h}, v_{s}\right)$ as

$$
\begin{aligned}
& v_{\psi}=f \arctan \left(\frac{v_{s}}{v_{h}}\right), \\
& v_{\phi}=f \arcsin \left(\frac{\sqrt{v_{h}^{2}+v_{s}^{2}}}{f}\right),
\end{aligned}
$$

where $v_{s} \equiv\langle s\rangle$. In the EWSB vacuum identified as $v_{\psi}=0$ (or $v_{s}=0$ ), one has

$$
v_{\phi}=f \arcsin \sqrt{\xi} .
$$

In this case, the nonlinearity exactly recovers that in the $\mathrm{MCHM}$ as

$$
\xi=\sin \left(v_{\phi} / f\right)
$$

Consider the physical Higgs excitation $h$ around $v$, one has

$$
\tan \left(\frac{h+v}{f}\right)=\frac{\sqrt{1-\xi} s_{h}+\sqrt{\xi} c_{h}}{\sqrt{1-\xi} c_{h}+\sqrt{\xi} s_{h}}
$$

where $s_{h}=\sin (h / f)$ and $c_{h}=\cos (h / f)$.

In the NMCHM, one can also change from the polar coordinate $(\phi, \psi)$ to the Cartesian $(h, s)$ coordinate with replacement rules:

$$
\mathcal{L}_{\phi} \rightarrow \frac{1}{1-\xi} \mathcal{L}_{H}, \quad \mathcal{L}_{\psi} \rightarrow \frac{1}{\xi} \mathcal{L}_{S},
$$

where $\mathcal{L}_{S} \equiv\left(\partial_{\mu} s\right)^{2} / 2$ and we have used Eq. (2.29).

It turns out that, in the $(h, s)$ field basis, the custodial preserving terms in the high-energy effective Lagrangian in Eq. (5.2) becomes

$\overline{\mathcal{L}}_{C}(\boldsymbol{\Sigma})=\frac{1}{2}\left(\left(\partial_{\mu} h\right)^{2}+\left(\partial_{\mu} s\right)^{2}+\frac{\left(h \partial_{\mu} h+s \partial_{\mu} s\right)^{2}}{f^{2}-h^{2}-s^{2}}\right)+\ldots$

where the first terms are kinetic terms and are not canonically normalized yet as

$$
\mathcal{L}_{\text {kin }}^{(h, s)}=\left(\partial_{\mu} h, \partial_{\mu} s\right) K(h, s)\left(\partial^{\mu} h, \partial^{\mu} s\right)^{T},
$$

where $K(h, s)$ is the matrix of kinetic terms with nondiagonal mixing terms of $\partial_{\mu} h \partial^{\mu} s$,

$$
K(h, s)=\frac{1}{2}\left(\begin{array}{cc}
\frac{f^{2}-s^{2}}{f^{2}-h^{2}-s^{2}} & \frac{h s}{f^{2}-h^{2}-s^{2}} \\
\frac{h s}{f^{2}-h^{2}-s^{2}} & \frac{f^{2}-h^{2}}{f^{2}-h^{2}-s^{2}}
\end{array}\right) .
$$

After EWSB, $v_{s}=\langle s\rangle \neq 0$ and $v_{h}=\langle h\rangle$, it can cause mixing and the eigenvalue matrix is [63]

$$
\begin{aligned}
\mathcal{L}_{\text {kin }}^{(h, s)} & =\partial_{\mu}(h, s)\left(\begin{array}{ll}
F_{11} & F_{12} \\
F_{21} & F_{22}
\end{array}\right) \partial^{\mu}\left(\begin{array}{l}
h \\
s
\end{array}\right) \\
& \rightarrow \lambda_{+}\left(\partial_{\mu} h\right)^{2}+\lambda_{-}\left(\partial_{\mu} s\right)^{2},
\end{aligned}
$$

where

$$
\begin{aligned}
& F_{11}=\frac{1}{2}\left(1+\frac{v_{h}^{2}}{f^{2}-v_{h}^{2}-v_{s}^{2}}\right), \\
& F_{12}=F_{21}=\frac{1}{2} \frac{v_{h} v_{s}}{f^{2}-v_{h}^{2}-v_{s}^{2}}, \\
& F_{22}=\frac{1}{2}\left(1+\frac{v_{s}^{2}}{f^{2}-v_{h}^{2}-v_{s}^{2}}\right) .
\end{aligned}
$$


The kinetic matrix can be diagonalized where the eigenvalues are

$$
\lambda_{+}=\frac{1}{2} \frac{1}{1-\frac{v_{h}^{2}+v_{s}^{2}}{f^{2}}}=\frac{1}{2 c_{\langle\phi\rangle}^{2}}, \quad \lambda_{-}=\frac{1}{2} .
$$

In the SM case, $s=\langle s\rangle=v_{s}=0$,

$$
s_{\langle\phi\rangle}=\sqrt{\xi}, \quad c_{\langle\phi\rangle}=\sqrt{1-\xi},
$$

one has [26]

$$
\lambda_{+}=\frac{1}{2(1-\xi)}, \quad \lambda_{-}=\frac{1}{2} .
$$

Then after rescaling, the physical singlets can be expressed as

$$
\begin{aligned}
& h \rightarrow \frac{v_{h} \frac{h}{\sqrt{2 \lambda_{+}}}-v_{s} \frac{s}{\sqrt{2 \lambda_{-}}}}{\sqrt{v_{h}^{2}+v_{s}^{2}}}=\frac{v_{h} h c_{\langle\phi\rangle}-v_{s} s}{\sqrt{v_{h}^{2}+v_{s}^{2}}} \stackrel{v_{s}=0}{=} h \sqrt{1-\xi}, \\
& s \rightarrow \frac{v_{h} \frac{s}{\sqrt{2 \lambda_{-}}}+v_{s} \frac{h}{\sqrt{2 \lambda_{+}}}}{\sqrt{v_{h}^{2}+v_{s}^{2}}}=\frac{v_{h} s+v_{s} h c_{\langle\phi\rangle}}{\sqrt{v_{h}^{2}+v_{s}^{2}}} \stackrel{v_{s}=0}{=} s .
\end{aligned}
$$

In the following, to simplify our physical results, we use this eigenbasis from the $\boldsymbol{\Sigma}$ parametrization to deduce the physical observables, such as the anomalous couplings of the Higgs boson to two gauge bosons, etc. In the EW vacuum, one needs to impose the

$$
h \rightarrow v+\sqrt{1-\xi} h, \quad s \rightarrow s .
$$

After redefinition, the kinetic terms are canonically normalized as

$$
\mathcal{L}_{\text {kin }}^{(h, s)} \rightarrow \mathcal{L}_{h}+\mathcal{L}_{s}=\frac{1}{2}\left[\left(\partial_{\mu} h\right)^{2}+\left(\partial_{\mu} s\right)^{2}\right],
$$

where the kinetic mixing terms are vanishing.

\section{The $\left(h_{4}, h_{5}\right)$ basis: Cartesian coordinates}

When going back to the original $\left(h_{4}, h_{5}\right)$ basis instead of the $(h, s)$ and $(\phi, \psi)$ basis, according to Eq. (2.15), refer to Table. I, the kinetic term in Eqs. (5.8) or (5.18) can be expressed more explicitly as

$$
\begin{aligned}
\mathcal{L}_{\text {kin }}^{\left(h_{4}, h_{5}\right)}= & \frac{\left(h_{4} \partial_{\mu} h_{4}+h_{5} \partial_{\mu} h_{5}\right)^{2}}{2\left(h_{4}^{2}+h_{5}^{2}\right)} \\
& +\frac{\left(h_{5} \partial_{\mu} h_{4}-h_{4} \partial_{\mu} h_{5}\right)^{2}}{2\left(h_{4}^{2}+h_{5}^{2}\right)^{2}} f^{2} \sin ^{2}\left(\frac{\sqrt{h_{4}^{2}+h_{5}^{2}}}{f}\right) \\
= & \left(\partial_{\mu} h_{4}, \partial_{\mu} h_{5}\right) K\left(h_{4}, h_{5}\right)\left(\partial^{\mu} h_{4}, \partial^{\mu} h_{5}\right)^{T},
\end{aligned}
$$

where $K\left(h_{4}, h_{5}\right)$ is the kinetic matrix with a nondiagonal mixing term of $\partial_{\mu} h_{4} \partial^{\mu} h_{5}$ as

$$
K\left(h_{4}, h_{5}\right)=\frac{1}{2}\left(\begin{array}{ll}
K_{11} & K_{12} \\
K_{21} & K_{22}
\end{array}\right),
$$

with

$$
\begin{aligned}
& K_{11}=\frac{h_{4}^{2}}{h_{4}^{2}+h_{5}^{2}}+\frac{h_{5}^{2} f^{2} \sin ^{2}\left(\frac{\sqrt{h_{4}^{2}+h_{5}^{2}}}{f}\right)}{\left(h_{4}^{2}+h_{5}^{2}\right)^{2}}, \\
& K_{12}=K_{21}=\frac{h_{4} h_{5}}{h_{4}^{2}+h_{5}^{2}}-\frac{h_{4} h_{5} f^{2} \sin ^{2}\left(\frac{\sqrt{h_{4}^{2}+h_{5}^{2}}}{f}\right)}{\left(h_{4}^{2}+h_{5}^{2}\right)^{2}}, \\
& K_{22}=\frac{h_{5}^{2}}{h_{4}^{2}+h_{5}^{2}}+\frac{h_{4}^{2} f^{2} \sin ^{2}\left(\frac{\sqrt{h_{4}^{2}+h_{5}^{2}}}{f}\right)}{\left(h_{4}^{2}+h_{5}^{2}\right)^{2}} .
\end{aligned}
$$

One can also transfer the vev from the $(\phi, \psi)$ basis to the $\left(h_{4}, h_{5}\right)$ basis,

$$
\frac{v}{f}=\sqrt{\xi}=\frac{v_{4}}{\sqrt{v_{4}^{2}+v_{5}^{2}}} \sin \left(\frac{\sqrt{v_{4}^{2}+v_{5}^{2}}}{f}\right)=\frac{v_{h}}{f},
$$

where $v_{4} \equiv\left\langle h_{4}\right\rangle$ and $v_{5} \equiv\left\langle h_{5}\right\rangle$ are vevs of $h_{4}$ and $h_{5}$, respectively.

In the absence of $h_{5}$, the kinetic term just recovers that of MCHM in the leading $p^{2}$ order effective Lagrangian of MCHM in $\Omega$ parametrization in Eq. (5.3). One can promote the $h_{4}$ to be the $S O(4)$ global invariants as

$$
|H|^{2}=h_{1}^{2}+h_{2}^{2}+h_{3}^{2}+h_{4}^{2}=(h+v)^{2},
$$

and relabel the singlet $h_{5}=\eta$. In this case, the kinetic terms in Eq. (5.29) are promoted to be more generic ones with

$$
\begin{aligned}
\mathcal{L}_{\text {kin }}^{(H, \eta)}= & \frac{f^{2} \sin ^{2}\left(\sqrt{|H|^{2}+\eta^{2}} / f\right)}{2\left(|H|^{2}+\eta^{2}\right)}\left[\left(\partial_{\mu} H\right)^{\dagger}\left(\partial^{\mu} H\right)+\partial_{\mu} \eta \partial^{\mu} \eta\right] \\
& +\frac{\left[\partial_{\mu}\left(|H|^{2}+\eta^{2}\right)\right]^{2}}{8\left(|H|^{2}+\eta^{2}\right)}\left(1-\frac{f^{2} \sin ^{2}\left(\sqrt{|H|^{2}+\eta^{2}} / f\right)}{|H|^{2}+\eta^{2}}\right) .
\end{aligned}
$$

It is also interesting to observe that the metric in front of the Higgs kinetic terms $\mathcal{L}=g_{a b} \partial_{\mu} h^{a} \partial h^{b} / 2$, can be expressed with a metric

$$
g_{a b}=\frac{f^{2} \sin ^{2}\left(\sqrt{|H|^{2}+\eta^{2}} / f\right)}{2\left(|H|^{2}+\eta^{2}\right)} \delta_{a b} .
$$

In the weak coupling limit $f \rightarrow \infty$, the second term disappears, while the first term just recovers the kinetic terms of the SM Higgs doublet and $\eta$

$$
\mathcal{L}_{\text {kin }}^{(H, \eta)} \stackrel{f \rightarrow \infty}{=} \frac{1}{2}\left[\left(\partial_{\mu} H\right)^{\dagger} \partial^{\mu} H+\partial_{\mu} \eta \partial^{\mu} \eta\right] .
$$


By gauging the theory with replacing $\partial_{\mu} \rightarrow D_{\mu}$, after EWSB, in the $f \rightarrow \infty$ limit, one would expect to obtain the Lagrangian of the SM effective field theory as a series of expansions. In the unitary gauge, $H^{\dagger} H=|H|^{2}=(v+h)^{2}$ and $\partial_{\mu}\left(H^{\dagger} H\right)=2(v+h) \partial_{\mu} h$.

\section{B. Higgs function in $(\phi, \psi)$ basis}

\section{CP-even case}

For $C P$-even operators at $\mathcal{O}\left(p^{2}\right)$ order, i.e., with at most two derivatives,

$$
\mathcal{L}_{\text {low }}^{p^{2}}=c_{C} \mathcal{L}_{C} \mathcal{F}_{C}+c_{T} \mathcal{L}_{T} \mathcal{F}_{T}+c_{H} \mathcal{L}_{H} \mathcal{F}_{H},
$$

where $\mathcal{L}_{T, C}$ are the leading-order low-energy $C P$-even chiral effective Lagrangians introduced in Eq. (C11) and $\mathcal{L}_{H}$ is the canonically normalized Higgs singlet kinetic term defined in Eq. (C9). For briefness, henceforth we have neglect the explicit $h$ dependence expression for $\mathcal{F}(h)$, instead by making the abbreviation $\mathcal{F}$. The multiplicative terms $\mathcal{F}_{n}$ in terms of the Higgs functions, are the generic polynomial functions of $h$. For the composite Higgs model, $\mathcal{F}_{n}$ is the trigonometric functions of $h / f$. For the NMCHM, the Higgs functions can be read directly from Eq. (4.1) through the matching below as

$$
\begin{aligned}
& \bar{c}_{C} \overline{\mathcal{L}}_{C} \equiv \mathcal{F}_{H} c_{H} \mathcal{L}_{H}+\mathcal{F}_{S} c_{S} \mathcal{L}_{S}+\mathcal{F}_{C} c_{C} \mathcal{L}_{C}, \\
& \bar{c}_{T} \overline{\mathcal{L}}_{T} \equiv \mathcal{F}_{T} c_{T} \mathcal{L}_{T},
\end{aligned}
$$

where

$$
\begin{aligned}
& \mathcal{F}_{H} c_{H}=\bar{c}_{C}, \quad \mathcal{F}_{S} c_{S}=\sin ^{2}\left(\frac{\phi}{f}\right) \bar{c}_{C}, \\
& \mathcal{F}_{C} c_{C}=\frac{4}{\xi} \sin ^{2}\left(\frac{\phi}{2 f}\right) \cos ^{2}\left(\frac{\psi}{f}\right) \bar{c}_{C}, \\
& \mathcal{F}_{T} c_{T}=-\frac{1}{\xi} \cos ^{4}\left(\frac{\psi}{f}\right) \sin ^{4}\left(\frac{\phi}{2 f}\right) \bar{c}_{T} .
\end{aligned}
$$

Assume that $v$ and $v_{s}$ are the expectation values of Higgs $h$ and singlet $s$ as

$v \equiv 2 f \arcsin \left(\frac{\sqrt{\xi}}{2} \sec \frac{v_{s}}{f}\right) \stackrel{v_{s}=0}{=} 2 f \arcsin \left(\frac{\sqrt{\xi}}{2}\right)$.

The Higgs function after expansion gives coefficients as

$$
\begin{aligned}
\mathcal{F}_{H} c_{H} & =\bar{c}_{H}, \\
\mathcal{F}_{C} c_{C} & =\bar{c}_{C}\left(1+2 a_{C} \frac{h}{v}+b_{C} \frac{h^{2}}{v^{2}}+\ldots\right), \\
\mathcal{F}_{T} c_{T} & =-\frac{\xi}{16} \bar{c}_{T}\left(1+2 a_{T} \frac{h}{v}+b_{T} \frac{h^{2}}{v^{2}}+\ldots\right),
\end{aligned}
$$

where $\mathcal{F}_{H}=1$ when $c_{H}=\bar{c}_{H}$ and

$$
\begin{aligned}
& a_{C}=\sqrt{c_{s}^{2}-\frac{1}{4}} \xi \stackrel{v_{s}=0}{=} \sqrt{1-\frac{\xi}{4}} \approx 1-\frac{\xi}{8}, \\
& b_{C}=c_{s}^{2}-\frac{1}{2} \xi \stackrel{v_{s}=0}{=} 1-\frac{1}{2} \xi \\
& a_{T}=\sqrt{4 c_{s}^{2}-\xi} \stackrel{v_{s}=0}{=} \sqrt{4-\xi} \approx 4-\frac{\xi}{2}, \\
& b_{T}=6 c_{s}^{2}-2 \xi \stackrel{v_{s}=0}{=} 6-2 \xi
\end{aligned}
$$

where we have defined $c_{s}=\cos \left(v_{s} / f\right)$. These result for $\mathcal{F}_{H}$ in the $\Omega$ parametrization after $\boldsymbol{\Sigma}$ decomposition, which just recovers exactly Eq. (7.4) in Ref. [49].

For the $C P$-even operators at $p^{4}$ order,

$$
\begin{aligned}
\mathcal{L}_{\text {low }}^{p^{4}}= & c_{B} \mathcal{L}_{B} \mathcal{F}_{B}+c_{W} \mathcal{L}_{W} \mathcal{F}_{W}+\sum_{n=1}^{26} c_{n} \mathcal{L}_{n} \mathcal{F}_{n} \\
& +c_{\square H} \mathcal{L}_{\square H} \mathcal{F}_{\square H}+c_{\Delta H} \mathcal{L}_{\Delta H} \mathcal{F}_{\Delta H} \\
& +c_{D H} \mathcal{L}_{D H} \mathcal{F}_{D H} .
\end{aligned}
$$

The low-energy $C P$-even chiral effective Lagrangian $\mathcal{L}_{B, W, n}$ is introduced in Eq. (C11) and $\mathcal{L}_{\square H, \triangle H, D H}$ is the higher order operator denoted in Eq. (C9).

From NMCHM, we find the Higgs functions as

$$
\begin{aligned}
& c_{B} \mathcal{F}_{B}=\bar{c}_{B}-4 \bar{c}_{B \Sigma}\left[1-\cos ^{2}\left(\frac{\psi}{f}\right) \sin ^{2}\left(\frac{\phi}{2 f}\right)\right], \\
& c_{W} \mathcal{F}_{W}=\bar{c}_{W}-4 \bar{c}_{W \boldsymbol{\Sigma}}\left[1-\cos ^{2}\left(\frac{\psi}{f}\right) \sin ^{2}\left(\frac{\phi}{2 f}\right)\right] \text {, } \\
& c_{D H} \mathcal{F}_{D H}^{(\phi)}=4 \xi^{2}\left(\bar{c}_{4}+\bar{c}_{5}\right), \\
& c_{D H} \mathcal{F}_{D H}^{(\psi)}=64 \xi^{2}\left(\bar{c}_{4}+\bar{c}_{5}\right) \sin ^{4}\left(\frac{\phi}{2 f}\right), \\
& c_{\square H} \mathcal{F}_{\square H}^{(\phi)}=-2 \xi \bar{c}_{6}, \\
& c_{\square H} \mathcal{F}_{\square H}^{(\psi)}=-8 \bar{c}_{6} \xi \sin ^{2}\left(\frac{\phi}{2 f}\right), \\
& c_{1} \mathcal{F}_{1}=\bar{c}_{1} \cos ^{2}\left(\frac{\psi}{f}\right) \sin ^{2}\left(\frac{\phi}{2 f}\right), \\
& c_{2} \mathcal{F}_{2}=\bar{c}_{2} \cos ^{2}\left(\frac{\psi}{f}\right) \sin ^{2}\left(\frac{\phi}{2 f}\right), \\
& c_{3} \mathcal{F}_{3}=2 \bar{c}_{3} \cos ^{2}\left(\frac{\psi}{f}\right) \sin ^{2}\left(\frac{\phi}{2 f}\right), \\
& c_{4} \mathcal{F}_{4}^{(\phi)}=\bar{c}_{2} \sqrt{\xi} \cos ^{2}\left(\frac{\psi}{f}\right) \sin \left(\frac{\phi}{f}\right), \\
& c_{4} \mathcal{F}_{4}^{(\psi)}=-2 \bar{c}_{2} \sqrt{\xi} \sin \left(\frac{2 \psi}{f}\right) \sin ^{2}\left(\frac{\phi}{2 f}\right), \\
& c_{5} \mathcal{F}_{5}^{(\phi)}=-2 \bar{c}_{3} \sqrt{\xi} \cos ^{2}\left(\frac{\psi}{f}\right) \sin \left(\frac{\phi}{f}\right), \\
& c_{5} \mathcal{F}_{5}^{(\psi)}=4 \bar{c}_{3} \sqrt{\xi} \sin \left(\frac{2 \psi}{f}\right) \sin ^{2}\left(\frac{\phi}{2 f}\right),
\end{aligned}
$$




$$
\begin{aligned}
& c_{6} \mathcal{F}_{6}=16 \bar{c}_{4} \cos ^{4}\left(\frac{\psi}{f}\right) \sin ^{4}\left(\frac{\phi}{2 f}\right) \\
& -2 \bar{c}_{6} \cos ^{2}\left(\frac{\psi}{f}\right) \sin ^{2}\left(\frac{\phi}{2 f}\right) \\
& \times\left[1-\cos ^{2}\left(\frac{\psi}{f}\right) \sin ^{2}\left(\frac{\phi}{2 f}\right)\right], \\
& c_{7} \mathcal{F}_{7}^{(\phi)}=-2 \bar{c}_{6} \sqrt{\xi} \cos ^{2}\left(\frac{\psi}{f}\right) \sin \left(\frac{\phi}{f}\right), \\
& c_{7} \mathcal{F}_{7}^{(\psi)}=4 \bar{c}_{6} \sqrt{\xi} \sin \left(\frac{2 \psi}{f}\right) \sin ^{2}\left(\frac{\phi}{2 f}\right), \\
& c_{8} \mathcal{F}_{8}^{(\phi)}=-16 \bar{c}_{5} \xi \cos ^{2}\left(\frac{\psi}{f}\right) \sin ^{2}\left(\frac{\phi}{2 f}\right) \\
& +4 \bar{c}_{6} \xi \cos ^{2}\left(\frac{\psi}{f}\right) \cos ^{2}\left(\frac{\phi}{2 f}\right), \\
& c_{8} \mathcal{F}_{8}^{(\psi)}=-64 \bar{c}_{5} \xi \cos ^{2}\left(\frac{\psi}{f}\right) \sin ^{4}\left(\frac{\phi}{2 f}\right) \\
& +16 \bar{c}_{6} \xi \sin ^{2}\left(\frac{\psi}{f}\right) \sin ^{2}\left(\frac{\phi}{2 f}\right), \\
& c_{9} \mathcal{F}_{9}=4 \bar{c}_{6} \cos ^{2}\left(\frac{\psi}{f}\right) \sin ^{2}\left(\frac{\phi}{2 f}\right), \\
& c_{10} \mathcal{F}_{10}^{(\phi)}=4 \bar{c}_{6} \sqrt{\xi} \cos ^{2}\left(\frac{\psi}{f}\right) \sin \left(\frac{\phi}{f}\right), \\
& c_{10} \mathcal{F}_{10}^{(\psi)}=-8 \bar{c}_{6} \sqrt{\xi} \sin \left(\frac{2 \psi}{f}\right) \sin ^{2}\left(\frac{\phi}{2 f}\right), \\
& c_{11} \mathcal{F}_{11}=16 \bar{c}_{5} \cos ^{4}\left(\frac{\psi}{f}\right) \sin ^{4}\left(\frac{\phi}{2 f}\right), \\
& c_{20} \mathcal{F}_{20}^{(\phi)}=-16 \bar{c}_{4} \xi \cos ^{2}\left(\frac{\psi}{f}\right) \sin ^{2}\left(\frac{\phi}{2 f}\right), \\
& c_{20} \mathcal{F}_{20}^{(\psi)}=-64 \bar{c}_{4} \xi \cos ^{2}\left(\frac{\psi}{f}\right) \sin ^{4}\left(\frac{\phi}{2 f}\right) .
\end{aligned}
$$

In the absence of the singlet $s$ (or $\psi=0$ ), the results recover the expression for $c_{n} \mathcal{F}_{n}$ custodial preserving operators for the MCHM in Table 1 in Ref. [49].

\section{CP-odd case}

The low-energy EW chiral Lagrangian that describes the $C P$-odd gauge-Goldstone and the gauge-scalar interactions can be written as

$$
\tilde{\mathcal{L}}_{\text {low }}=\tilde{\mathcal{L}}_{\text {low }}^{p^{2}}+\tilde{\mathcal{L}}_{\text {low }}^{p^{4}},
$$

where

$$
\begin{aligned}
& \tilde{\mathcal{L}}_{\text {low }}^{p^{2}}=c_{\tilde{T}} \mathcal{L}_{\tilde{T}} \mathcal{F}_{\tilde{T}}, \\
& \tilde{\mathcal{L}}_{\text {low }}^{p^{4}}=\mathcal{L}_{\tilde{B}} \mathcal{F}_{\tilde{B}}+\mathcal{L}_{\tilde{W}} \mathcal{F}_{\tilde{W}}+\sum_{n=1}^{16} c_{\tilde{n}} \mathcal{L}_{\tilde{n}} \mathcal{F}_{\tilde{n}},
\end{aligned}
$$

where $\mathcal{F}_{\tilde{n}}$ encoded a generic dependence on $h$ but without derivative of $h$. The low-energy $C P$-odd chiral effective Lagrangian $\mathcal{L}_{\tilde{T}, \tilde{B}, \tilde{W}, \tilde{n}}$ are introduced in Eq. (C15) or more explicitly shown in Eq. 482 in Appendix. C.

From the above relations, we read the Higgs functions as

$$
\begin{aligned}
c_{\tilde{T}} \mathcal{F}_{\tilde{T}}= & -\frac{1}{\xi} \cos ^{2}\left(\frac{\psi}{f}\right) \sin ^{2}\left(\frac{\phi}{2 f}\right) \bar{c}_{\tilde{T}}, \\
c_{\tilde{B}} \mathcal{F}_{\tilde{B}}= & -4 \bar{c}_{\tilde{B} \Sigma}\left[1-\cos ^{2}\left(\frac{\psi}{f}\right) \sin ^{2}\left(\frac{\phi}{2 f}\right)\right] \\
& +4 \bar{c}_{\tilde{2}} \cos ^{4}\left(\frac{\psi}{f}\right) \sin ^{4}\left(\frac{\phi}{2 f}\right), \\
c_{\tilde{W}} \mathcal{F}_{\tilde{W}}= & \bar{c}_{\tilde{W}}-4 \bar{c}_{\tilde{W} \Sigma}\left[1-\cos ^{2}\left(\frac{\psi}{f}\right) \sin ^{2}\left(\frac{\phi}{2 f}\right)\right] \\
& -4 \bar{c}_{\tilde{2}} \cos ^{4}\left(\frac{\psi}{f}\right) \sin ^{4}\left(\frac{\phi}{2 f}\right), \\
c_{\tilde{1}} \mathcal{F}_{\tilde{1}}= & \frac{1}{2} \bar{c}_{\tilde{1}} \cos ^{2}\left(\frac{\psi}{f}\right) \sin ^{2}\left(\frac{\phi}{2 f}\right), \\
c_{\tilde{2}} \mathcal{F}_{\tilde{2}}= & 2 \bar{c}_{\tilde{2}} \sqrt{\xi} \cos ^{4}\left(\frac{\psi}{f}\right) \cos \left(\frac{\phi}{2 f}\right) \sin ^{3}\left(\frac{\phi}{2 f}\right), \\
c_{\tilde{3}} \mathcal{F}_{\tilde{3}}= & 4 \bar{c}_{\tilde{2}} \sqrt{\xi} \cos ^{4}\left(\frac{\psi}{f}\right) \cos \left(\frac{\phi}{2 f}\right) \sin ^{3}\left(\frac{\phi}{2 f}\right) .
\end{aligned}
$$

When the $C P$-odd singlet ( $s$ or $h_{5}$ ) is absent, i.e., $\psi=0$, this just recovers the results in Table 1 in Ref. [52].

\section{Higgs function in $(h, s)$ basis}

\section{CP-even case}

From above equations in Eq. (5.2), or with the aid of Eq. (5.39), we can transform them from the $\Omega$ to $\boldsymbol{\Sigma}$ parametrization, by making the redefinition $f \rightarrow f / 2$ (or $\xi \rightarrow 4 \xi$ ) and $\psi \rightarrow \psi / 2$ as in Eq. (2.25). In NMCHM, the Higgs functions can be read directly from Eq. (4.1) through the matching below as

$$
\begin{aligned}
& \bar{c}_{C} \overline{\mathcal{L}}_{C} \equiv \mathcal{F}_{H} c_{H} \mathcal{L}_{H}+\mathcal{F}_{S} c_{S} \mathcal{L}_{S}+\mathcal{F}_{C} c_{C} \mathcal{L}_{C}, \\
& \bar{c}_{T} \overline{\mathcal{L}}_{T} \equiv \mathcal{F}_{T} c_{T} \mathcal{L}_{T},
\end{aligned}
$$

from which, we can read the Higgs functions corresponding to the Lagrangians, respectively, as

$$
\begin{aligned}
& \mathcal{F}_{H} c_{H}=\bar{c}_{C}, \quad \mathcal{F}_{S} c_{S}=\frac{1}{4} \sin ^{2}\left(\frac{2 \phi}{f}\right) \bar{c}_{C}, \\
& \mathcal{F}_{C} c_{C}=\frac{1}{\xi} \sin ^{2}\left(\frac{\phi}{f}\right) \cos ^{2}\left(\frac{\psi}{f}\right) \bar{c}_{C}, \\
& \mathcal{F}_{T} c_{T}=-\frac{1}{4 \xi} \cos ^{4}\left(\frac{\psi}{f}\right) \sin ^{4}\left(\frac{\phi}{f}\right) \bar{c}_{T},
\end{aligned}
$$

where $\mathcal{L}_{H}$ is defined in Eq. (C9). 
Assume that $v$ and $v_{s}$ are the expectation values of Higgs $\phi \rightarrow h+v$ and singlet $\psi \rightarrow s+v_{s}$ as

$$
v_{h} \equiv f \arcsin \left(\sqrt{\xi} \sec \frac{v_{s}}{f}\right) \stackrel{v_{s}=0}{=} f \arcsin (\sqrt{\xi}) .
$$

The Higgs function after expansion in the series up to the order $(h / v)^{2}$ gives expansion coefficients as

$$
\begin{aligned}
\mathcal{F}_{H} c_{H} & =\bar{c}_{H}, \\
\mathcal{F}_{C} c_{C} & =\bar{c}_{C}\left(1+2 a_{C} \frac{h}{v}+b_{C} \frac{h^{2}}{v^{2}}+\ldots\right), \\
\mathcal{F}_{T} c_{T} & =-\frac{\xi}{4} \bar{c}_{T}\left(1+2 a_{T} \frac{h}{v}+b_{T} \frac{h^{2}}{v^{2}}+\ldots\right),
\end{aligned}
$$

where $\mathcal{F}_{H}=1$ when $c_{H}=\bar{c}_{H}$ and

$$
\begin{aligned}
& a_{C}=\sqrt{c_{s}^{2}-\xi} \stackrel{v_{s}=0}{=} \sqrt{1-\xi} \approx 1-\frac{\xi}{2}, \\
& b_{C}=c_{s}^{2}-2 \xi \stackrel{v_{s}=0}{=} 1-2 \xi ; \\
& a_{T}=2 \sqrt{c_{s}^{2}-\xi} \stackrel{v_{s}=0}{=} 2 \sqrt{1-\xi} \approx 2-\xi, \\
& b_{T}=6 c_{s}^{2}-8 \xi^{v_{s}=0}=0-8 \xi,
\end{aligned}
$$

where we have defined $c_{s} \equiv \cos \left(v_{s} / f\right)$. These result for $\mathcal{F}_{H}$ in $\boldsymbol{\Sigma}$ parametrization, in the absence of the singlet $s$, i.e., $v_{s}=0$, just exactly recovers those in the MCHM $[19,49]$. For $\mathcal{F}_{T}, c_{T}$ is related to the $\mathrm{EW}$ oblique $T$ parameter as denoted in Eq. (6.11) after EWSB $\langle h\rangle=v$. Due to the $-\xi / 4$ factor in front of the Higgs function $\mathcal{F}_{T}$ is suppressed at leading $\mathcal{O}\left(p^{2}\right)$ order.

For the Higgs functions associated with $C P$-even operators at the order $\left(p^{4}\right)$, one has

$$
\begin{aligned}
c_{B} \mathcal{F}_{B} & \approx \bar{c}_{B}-4 \bar{c}_{B \Sigma}\left[1-\xi^{2}\left(1+\frac{h}{v}\right)-\xi(1-\xi)\left(1+\frac{h}{v}\right)^{2}\right], \\
c_{W} \mathcal{F}_{W} & \approx \bar{c}_{W}-4 \bar{c}_{W \Sigma}\left[1-\xi^{2}\left(1+\frac{h}{v}\right)-\xi(1-\xi)\left(1+\frac{h}{v}\right)^{2}\right], \\
c_{D H} \mathcal{F}_{D H}^{(\phi)} & \approx 64 \xi^{2}\left(\bar{c}_{4}+\bar{c}_{5}\right), \\
c_{D H} \mathcal{F}_{D H}^{(\psi)} & \approx 1024\left(\bar{c}_{4}+\bar{c}_{5}\right) \xi^{4}\left[\left(1+\frac{h}{v}\right)^{4}+2\left(1+\frac{h}{v}\right)^{2} \frac{s^{2}}{\left.v^{2}\right],}\right. \\
c_{\square H} \mathcal{F}_{\square H}^{(\phi)} & \approx-8 \bar{c}_{6} \xi, \\
c_{\square H} \mathcal{F}_{\square H}^{(\psi)} & \approx-8 \bar{c}_{6} \xi\left[\xi\left(1+\frac{h}{v}\right)+(1-\xi)\left(1+\frac{h}{v}\right)^{2}+\frac{s^{2}}{v^{2}}\right], \\
c_{1,2} \mathcal{F}_{1,2} & \approx \bar{c}_{1,2} \xi\left[\xi\left(1+\frac{h}{v}\right)+(1-\xi)\left(1+\frac{h}{v}\right)^{2}\right], \\
c_{3} \mathcal{F}_{3} & \approx 2 \bar{c}_{3} \xi\left[\xi\left(1+\frac{h}{v}\right)+(1-\xi)\left(1+\frac{h}{v}\right)^{2}\right],
\end{aligned}
$$

$$
\begin{aligned}
& c_{4} \mathcal{F}_{4}^{(\phi)} \approx 2 \bar{c}_{2} \xi\left[\xi+(2-\xi)\left(1+\frac{h}{v}\right)-\xi\left(1+\frac{h}{v}\right)^{3}\right], \\
& c_{4} \mathcal{F}_{4}^{(\psi)} \approx-8 \bar{c}_{2} \xi^{3 / 2}\left(1+\frac{h}{v}\right) \frac{s}{v}, \\
& c_{5} \mathcal{F}_{5}^{(\phi)} \approx-4 \bar{c}_{3} \xi\left[\xi+(2-\xi)\left(1+\frac{h}{v}\right)-\xi\left(1+\frac{h}{v}\right)^{3}\right], \\
& c_{4} \mathcal{F}_{4}^{(\psi)} \approx 16 \bar{c}_{3} \xi^{3 / 2}\left(1+\frac{h}{v}\right) \frac{s}{v}, \\
& c_{6} \mathcal{F}_{6} \approx 16 \bar{c}_{4} \xi^{2}\left(1+\frac{h}{v}\right)^{4}-2 \bar{c}_{6} \xi\left(1+\frac{h}{v}\right) \\
& \times\left[\xi+(1-\xi)\left(1+\frac{h}{v}\right)-\xi\left(1+\frac{h}{v}\right)^{3}\right], \\
& c_{7} \mathcal{F}_{7}^{(\phi)} \approx-4 \bar{c}_{6} \xi\left[\xi+(2-\xi)\left(1+\frac{h}{v}\right)-\xi\left(1+\frac{h}{v}\right)^{3}\right] \text {, } \\
& c_{7} \mathcal{F}_{7}^{(\psi)} \approx 16 \bar{c}_{6} \xi^{3 / 2}\left(1+\frac{h}{v}\right) \frac{s}{v}, \\
& c_{8} \mathcal{F}_{8}^{(\phi)} \approx-64 \bar{c}_{5} \xi^{2}\left[\xi\left(1+\frac{h}{v}\right)+(1-\xi)\left(1+\frac{h}{v}\right)^{2}\right] \\
& +16 \bar{c}_{6} \xi\left[1-\xi^{2}\left(1+\frac{h}{v}\right)-\xi(1-\xi)\left(1+\frac{h}{v}\right)^{2}\right], \\
& c_{8} \mathcal{F}_{8}^{(\psi)} \approx-64 \bar{c}_{5} \xi^{3}\left(1+\frac{h}{v}\right)^{4}+16 \bar{c}_{6} \xi^{2} \frac{s^{2}}{v^{2}}, \\
& c_{9} \mathcal{F}_{9} \approx 4 \bar{c}_{6} \xi\left[\xi\left(1+\frac{h}{v}\right)+(1-\xi)\left(1+\frac{h}{v}\right)^{2}\right], \\
& c_{10} \mathcal{F}_{10}^{(\phi)} \approx 8 \bar{c}_{6} \xi\left[\xi+(2-\xi)\left(1+\frac{h}{v}\right)-\xi\left(1+\frac{h}{v}\right)^{3}\right] \text {, } \\
& c_{10} \mathcal{F}_{10}^{(\psi)} \approx-32 \bar{c}_{6} \xi^{3 / 2} \frac{s}{v}, \\
& c_{11} \mathcal{F}_{11} \approx 16 \bar{c}_{5} \xi^{2}\left(1+\frac{h}{v}\right)^{4}, \\
& c_{20} \mathcal{F}_{20}^{(\phi)} \approx-16 \bar{c}_{4} \xi^{2}\left(1+\frac{h}{v}\right)^{2}, \\
& c_{20} \mathcal{F}_{20}^{(\psi)} \approx-64 \bar{c}_{4} \xi^{3}\left(1+\frac{h}{v}\right)^{2} \frac{s^{2}}{v^{2}}-64 \bar{c}_{4} \xi^{3}\left(1+\frac{h}{v}\right)^{4} .
\end{aligned}
$$

The $C P$-even anomalous couplings for HEFT from NMCHM can be obtained from expanding the Higgs functions as in the series up to order $(h / v)^{2}$ as [45]

$$
\mathcal{F}_{n}=1+2 \frac{\hat{a}_{n}}{c_{n}} \frac{h}{v}+\frac{\hat{b}_{n}}{c_{n}} \frac{h^{2}}{v^{2}}+\cdots,
$$

where $c_{n}$ are the global operator coefficients, $\hat{a}_{n} \equiv a_{n} c_{n}$ and $\hat{b}_{n} \equiv b_{n} c_{n} . c_{n}$ is independent of the Higgs functions $\mathcal{F}_{n}$ while $a_{n}$ and $b_{n}$ are related to anomalous couplings beyond the SM, which are related to a three- or four-point 
function, respectively, e.g., a single or double Higgs scalar, couplings to two gauge bosons.

For the NMCHM, we find the explicit expression of all nonvanishing coefficients as

$$
\begin{aligned}
& c_{B}=\bar{c}_{B}-4(1-\xi) \bar{c}_{B \Sigma}, \\
& \hat{a}_{B}=4 \xi \sqrt{c_{s}^{2}-\xi} \bar{c}_{B \Sigma} \approx 2 \xi(2-\xi) \bar{c}_{B \Sigma}, \\
& \hat{b}_{B}=4 \xi\left(c_{s}^{2}-2 \xi\right) \bar{c}_{B \Sigma} \approx 4 \xi(1-2 \xi) \bar{c}_{B \Sigma}, \\
& c_{W}=\bar{c}_{W}-4(1-\xi) \bar{c}_{W \mathbf{\Sigma}} \text {, } \\
& \hat{a}_{W}=4 \xi \sqrt{c_{s}^{2}-\xi} \bar{c}_{W \Sigma} \approx 2 \xi(2-\xi) \bar{c}_{W \mathbf{\Sigma}}, \\
& \hat{b}_{W}=4 \xi\left(c_{s}^{2}-2 \xi\right) \bar{c}_{W \Sigma} \approx 4 \xi(1-2 \xi) \bar{c}_{W \Sigma}, \\
& c_{D H}=64 \xi^{2}\left(\bar{c}_{4}+\bar{c}_{5}\right), \hat{a}_{D H}=0, \hat{b}_{D H}=0 \text {; } \\
& c_{\square H}=-8 \xi \bar{c}_{6}, \hat{a}_{\square H}=0, \hat{b}_{\square H}=0 ; \\
& c_{\square S}=-8 \xi \frac{1}{c_{S}^{2}} \bar{c}_{6} \approx-8 \xi \bar{c}_{6}, \\
& \hat{a}_{\square S}=-8 \xi \frac{1}{c_{s}^{2}} \sqrt{c_{s}^{2}-\xi} \bar{c}_{6} \approx-4 \xi(2-\xi) \bar{c}_{6}, \\
& \hat{b}_{\square S}=-8 \xi\left(1-2 \xi \frac{1}{c_{s}^{2}}\right) \bar{c}_{6} \approx-8 \xi(1-2 \xi) \bar{c}_{6} ; \\
& c_{1,2}=\xi \bar{c}_{1,2}, \\
& \hat{a}_{1,2}=\xi \sqrt{c_{s}^{2}-\xi} \bar{c}_{1,2} \approx \xi(2-\xi) / 2 \bar{c}_{1,2}, \\
& \hat{b}_{1,2}=\xi\left(c_{s}^{2}-2 \xi\right) \bar{c}_{1,2} \approx \xi(1-2 \xi) \bar{c}_{1,2} ; \\
& c_{3}=2 \xi \bar{c}_{3}, \\
& \hat{a}_{3}=2 \xi \sqrt{c_{s}^{2}-\xi} \bar{c}_{3} \approx \xi(2-\xi) \bar{c}_{3}, \\
& \hat{b}_{3}=2 \xi\left(c_{s}^{2}-2 \xi\right) \bar{c}_{3} \approx 2 \xi(1-2 \xi) \bar{c}_{3} \text {; } \\
& c_{4}=4 \xi \sqrt{c_{s}^{2}-\xi} \bar{c}_{2} \approx 2 \xi(2-\xi) \bar{c}_{2}, \\
& \hat{a}_{4}=2 \xi\left(c_{s}^{2}-2 \xi\right) \bar{c}_{2} \approx 2 \xi(1-2 \xi) \bar{c}_{2}, \\
& \hat{b}_{4}=-8 \xi \sqrt{c_{s}^{2}-\xi} \bar{c}_{2} \approx-8 \xi^{2} \bar{c}_{2} ; \\
& c_{5}=-8 \xi \sqrt{c_{s}^{2}-\xi} \bar{c}_{3} \approx-4 \xi(2-\xi) \bar{c}_{3}, \\
& \hat{a}_{5}=-4 \xi\left(c_{s}^{2}-2 \xi\right) \bar{c}_{3} \approx-4 \xi(1-2 \xi) \bar{c}_{3}, \\
& \hat{b}_{5}=16 \xi^{2} \sqrt{c_{s}^{2}-\xi} \bar{c}_{3} \approx 16 \xi^{2} \bar{c}_{3} ; \\
& c_{6}=16 \xi^{2} \bar{c}_{4}-2 \xi(1-\xi) \bar{c}_{6} \text {, } \\
& \hat{a}_{6}=32 \xi^{2} \sqrt{c_{s}^{2}-\xi} \bar{c}_{4}-2 \xi(1-2 \xi) \sqrt{c_{s}^{2}-\xi} \bar{c}_{6} \\
& \approx 32 \xi^{2} \bar{c}_{4}-\xi(2-5 \xi) \bar{c}_{6}, \\
& \hat{b}_{6}=32 \xi^{2}\left(3 c_{s}^{2}-4 \xi\right) \bar{c}_{4}+2 \xi\left[2 \xi(1-4 \xi)-(1-6 \xi) c_{s}^{2}\right] \bar{c}_{6} \\
& \approx 96 \xi^{2} \bar{c}_{4}-2 \xi(1-8 \xi) \bar{c}_{6} \text {; } \\
& c_{7}=-8 \xi \sqrt{c_{s}^{2}-\xi} \bar{c}_{6} \approx-4 \xi(2-\xi) \bar{c}_{6},
\end{aligned}
$$

$$
\begin{aligned}
\hat{a}_{7} & =-4 \xi\left(c_{s}^{2}-2 \xi\right) \bar{c}_{6} \approx-4 \xi(1-2 \xi) \bar{c}_{6}, \\
\hat{b}_{7} & =16 \xi^{2} \sqrt{c_{s}^{2}-\xi} \bar{c}_{6} \approx 16 \xi^{2} \bar{c}_{6} \\
c_{8} & =-64 \xi^{2} \bar{c}_{5}+16 \xi\left(c_{s}^{2}-\xi\right) \bar{c}_{6} \approx-64 \xi^{2} \bar{c}_{5}+16 \xi(1-\xi) \bar{c}_{6} \\
\hat{a}_{8} & =-64 \xi^{2} \sqrt{c_{s}^{2}-\xi} \bar{c}_{5}-16 \xi^{2} \sqrt{c_{s}^{2}-\xi} \bar{c}_{6} \\
& \approx-32(2-\xi) \xi^{2} \bar{c}_{5}-8(2-\xi) \xi^{2} \bar{c}_{6}, \\
\hat{b}_{8} & =-64 \xi^{2}\left(c_{s}^{2}-2 \xi\right) \bar{c}_{5}-16 \xi^{2}\left(c_{s}^{2}-2 \xi\right) \bar{c}_{6} \\
& \approx-64(1-2 \xi) \xi^{2} \bar{c}_{5}-16(1-2 \xi) \xi^{2} \bar{c}_{6} \\
c_{9} & =4 \xi \bar{c}_{6} \\
\hat{a}_{9} & =4 \xi \sqrt{c_{s}^{2}-\xi} \bar{c}_{6} \approx 2 \xi(2-\xi) \bar{c}_{6}, \\
\hat{b}_{9} & =4 \xi\left(c_{s}^{2}-2 \xi\right) \bar{c}_{6} \approx 4 \xi(1-2 \xi) \bar{c}_{6} \\
c_{10} & =16 \xi \sqrt{c_{s}^{2}-\xi} \bar{c}_{6} \approx 8 \xi(2-\xi) \bar{c}_{6} \\
\hat{a}_{10} & =8 \xi\left(c_{s}^{2}-2 \xi\right) \bar{c}_{6} \approx 8 \xi(1-2 \xi) \bar{c}_{6} \\
\hat{b}_{10} & =-32 \xi^{2} \sqrt{c_{s}^{2}-\xi} \bar{c}_{6} \approx-32 \xi^{2} \bar{c}_{6} \\
c_{11} & =16 \xi^{2} \bar{c}_{5}, \\
\hat{a}_{11} & =32 \xi^{2} \sqrt{c_{s}^{2}-\xi} \bar{c}_{5} \approx 32 \xi^{2} \bar{c}_{5} \\
\hat{b}_{11} & =32 \xi^{2}\left(3 c_{s}^{2}-4 \xi\right) \bar{c}_{5} \approx 96 \xi^{2} \bar{c}_{5} \\
c_{20} & =-64 \xi^{2} \bar{c}_{4}, \\
\hat{a}_{20} & =-64 \xi^{2} \sqrt{c_{s}^{2}-\xi} \bar{c}_{4} \approx-64 \xi^{2} \bar{c}_{4} \\
\hat{b}_{20} & =-64 \xi^{2}\left(c_{s}^{2}-2 \xi\right) \bar{c}_{4} \approx-64 \xi^{2} \bar{c}_{4} \\
w & (5.55)
\end{aligned}
$$

where we have denoted $c_{s} \equiv \cos \left(v_{s} / f\right)$, and $\approx$ means all terms at an order higher than $\mathcal{O}\left(\xi^{2}\right)$ are dropped.

\section{CP-odd case}

We can transform Eq. (5.47) from the $\Omega$ to the $\boldsymbol{\Sigma}$, parametrization, by making the redefinition $f \rightarrow f / 2$ (or $\xi \rightarrow 4 \xi$ ) and $\psi \rightarrow \psi / 2$ as in Eq. (2.25). In the canonically normalized basis of $h, s$, after EWSB, i.e., by making replacement for $h$ and $s$ as $h \rightarrow \sqrt{1-\xi} h+v_{h}$ and $s \rightarrow s$, expanding $\xi$ and $s$ up to second order, the Higgs functions associated with $C P$-odd operators at the order $\left(p^{4}\right)$ become

$$
\begin{aligned}
c_{\tilde{T}} \mathcal{F}_{\tilde{T}} \approx & -\frac{1}{4} \bar{c}_{\tilde{T}}\left[1+2 \sqrt{1-\xi} \frac{h^{2}}{v^{2}}+(1-2 \xi) \frac{h^{4}}{v^{4}}\right], \\
c_{\tilde{B}} \mathcal{F}_{\tilde{B}} \approx & -4 \bar{c}_{\tilde{B}}\left[1-\xi^{2}\left(1+\frac{h}{v}\right)-(1-\xi) \xi\left(1+\frac{h}{v}\right)^{2}\right] \\
& +4 \bar{c}_{\tilde{2}} \xi^{2}\left(1+\frac{h}{v}\right)^{4}, \\
c_{\tilde{W}} \mathcal{F}_{\tilde{W}} \approx & \bar{c}_{\tilde{W}}-4 \bar{c}_{\tilde{W}}\left[1-\xi^{2}\left(1+\frac{h}{v}\right)\right. \\
& \left.-(1-\xi) \xi\left(1+\frac{h}{v}\right)^{2}\right]+4 \bar{c}_{\tilde{2}} \xi^{2}\left(1+\frac{h}{v}\right)^{4},
\end{aligned}
$$




$$
\begin{aligned}
& c_{1} \mathcal{F}_{\tilde{1}} \approx \frac{1}{2} c_{\tilde{1}} \xi\left(1+\frac{h}{v}\right)\left[\xi+(1-\xi)\left(1+\frac{h}{v}\right)\right], \\
& c_{2} \mathcal{F}_{\tilde{2}} \approx 4 c_{\tilde{2}} \xi^{2}\left(1+\frac{h}{v}\right)\left[\left(1+\frac{h}{v}\right)^{2}-\frac{1}{2} \frac{s^{2}}{v^{2}}\right] \\
& c_{3} \mathcal{F}_{\tilde{3}} \approx 8 c_{\tilde{2}} \xi^{2}\left(1+\frac{h}{v}\right)\left[\left(1+\frac{h}{v}\right)^{2}-\frac{1}{2} \frac{s^{2}}{v^{2}}\right]
\end{aligned}
$$

The $C P$-odd anomalous couplings for the NMCHM can be obtained from expanding the $C P$-odd Higgs functions in Eq. (5.57) by

$$
\mathcal{F}_{\tilde{n}}=1+2 \frac{\hat{a}_{\tilde{n}}}{c_{\tilde{n}}} \frac{h}{v}+\frac{\hat{b}_{\tilde{n}}}{c_{\tilde{n}}} \frac{h^{2}}{v^{2}}+\cdots
$$

where $\hat{a}_{\tilde{n}} \equiv a_{\tilde{n}} c_{\tilde{n}}$, and $\hat{b}_{\tilde{n}} \equiv b_{\tilde{n}} c_{\tilde{n}}$. We find the nonvanishing coefficients $\hat{a}_{\tilde{n}}$ from NMCHM to be

$$
\begin{aligned}
& \hat{c}_{\tilde{T}}=-\frac{1}{4} \bar{c}_{\tilde{T}} \\
& a_{\tilde{T}}=\sqrt{c_{s}^{2}-\xi} \stackrel{v_{s}=0}{=} \sqrt{1-\xi} \approx 1-\frac{1}{2} \xi, \\
& b_{\tilde{T}}=c_{s}^{2}-2 \xi \stackrel{v_{s}=0}{=} 1-2 \xi \text {, } \\
& c_{\tilde{B}}=4\left[-(1-\xi) \bar{c}_{\tilde{B} \boldsymbol{\Sigma}}+\xi^{2} \bar{c}_{\tilde{2}}\right] \text {, } \\
& \hat{a}_{\tilde{B}}=4 \xi \sqrt{c_{s}^{2}-\xi} \bar{c}_{\tilde{B} \mathbf{\Sigma}}+8 \xi^{2} \sqrt{c_{s}^{2}-\xi} \bar{c}_{\tilde{2}} \\
& \approx 2 \xi\left[(2-\xi) \bar{c}_{\tilde{B} \Sigma}+4 \xi \bar{c}_{\tilde{2}}\right], \\
& \hat{b}_{\tilde{B}}=4 \xi\left(c_{s}^{2}-2 \xi\right) \bar{c}_{\tilde{B} \Sigma}+8 \xi^{2}\left(3 c_{s}^{2}-4 \xi\right) \bar{c}_{\tilde{2}} \\
& \approx 4 \xi\left[(1-2 \xi) \bar{c}_{\tilde{B} \Sigma}+6 \xi \bar{c}_{\tilde{2}}\right], \\
& c_{\tilde{W}}=\bar{c}_{\tilde{W}}+4\left[-(1-\xi) \bar{c}_{\tilde{W} \Sigma}+\xi^{2} \bar{c}_{\tilde{2}}\right] \text {, } \\
& \hat{a}_{\tilde{W}}=4 \xi \sqrt{c_{s}^{2}-\xi} \bar{c}_{\tilde{W} \boldsymbol{\Sigma}}+8 \xi^{2} \sqrt{c_{s}^{2}-\xi} \bar{c}_{\tilde{2}} \\
& \approx 2 \xi\left[(2-\xi) \bar{c}_{\tilde{W} \Sigma}+4 \xi \bar{c}_{\tilde{2}}\right], \\
& \hat{b}_{\tilde{W}}=4 \xi\left(c_{s}^{2}-2 \xi\right) \bar{c}_{\tilde{W} \Sigma}+8 \xi^{2}\left(3 c_{s}^{2}-4 \xi\right) \bar{c}_{\tilde{2}} \\
& \approx 4 \xi\left[(1-2 \xi) \bar{c}_{\tilde{W} \Sigma}+6 \xi \bar{c}_{\tilde{2}}\right], \\
& c_{\tilde{1}}=\frac{1}{2} \xi \bar{c}_{\tilde{1}}, \\
& \hat{a}_{\tilde{1}}=\frac{1}{2} \xi \sqrt{c_{s}^{2}-\xi} \bar{c}_{\tilde{1}} \approx \frac{1}{4} \xi(2-\xi) \bar{c}_{\tilde{1}}, \\
& \hat{b}_{\tilde{1}}=\frac{1}{2} \xi\left(c_{s}^{2}-2 \xi\right) \bar{c}_{\tilde{1}} \approx \frac{1}{2} \xi(1-2 \xi) \bar{c}_{\tilde{1}} ; \\
& c_{\tilde{2}}=4 \xi^{2} \sqrt{c_{s}^{2}-\xi} \bar{c}_{\tilde{2}} \approx 4 \xi^{2} \bar{c}_{\tilde{2}}, \\
& \hat{a}_{\tilde{2}}=2\left(3 c_{s}^{2}-4 \xi\right) \xi^{2} \bar{c}_{\tilde{2}} \approx 6 \xi^{2} \bar{c}_{\tilde{2}} \text {, } \\
& \hat{b}_{\tilde{2}}=4 \xi^{2}\left(3 c_{s}^{2}-8 \xi\right) \sqrt{c_{s}^{2}-\xi} \bar{c}_{\tilde{2}} \approx 12 \xi^{2} \bar{c}_{\tilde{2}} ; \\
& c_{\tilde{3}}=8 \xi^{2} \sqrt{c_{s}^{2}-\xi} \bar{c}_{\tilde{2}} \approx 8 \xi^{2} \bar{c}_{\tilde{2}} \text {, }
\end{aligned}
$$

$$
\begin{aligned}
& \hat{a}_{\tilde{3}}=4 \xi^{2}\left(3 c_{s}^{2}-4 \xi\right) \bar{c}_{\tilde{2}} \approx 12 \xi^{2} \bar{c}_{\tilde{2}}, \\
& \hat{b}_{\tilde{3}}=8 \xi^{2} \sqrt{c_{s}^{2}-\xi}\left(3 c_{s}^{2}-8 \xi\right) \bar{c}_{\tilde{2}} \approx 24 \xi^{2} \bar{c}_{\tilde{2}}
\end{aligned}
$$

where we have defined $c_{s} \equiv \cos \left(v_{s} / f\right)$, and dropped all terms at orders higher than $\mathcal{O}\left(\xi^{2}\right)$ in the approximation.

\section{Higgs functions in curved field space}

In the above section, we have obtained the Higgs functions from $\boldsymbol{\Sigma}$ decomposition of a high-energy effective Lagrangian. One founds that in the $\left(h_{4}, h_{5}\right)$ basis, such as Eq. (5.34), the kinetic term of the Higgs is not canonically normalized, unless in the $f \rightarrow \infty$ limit. This motives us to consider the physical meaning and origins for the Higgs function, which is an intriguing problem. We want to address the connection between the Higgs function and the intrinsic coset spacetime, which shows exactly how the Higgs functions are emergent due to the hierarchy between EW scale $v$ and strong symmetry breaking scale $f$. Inspired by the geometric viewpoint on the PNGB effective field theory in Ref. [39], we are reproducing, extending, and discussing the results in Ref. [58-60]. We consider not only fundamental but also adjoint representations for PNGBs in NMCHM. Moreover, we invest not only intrinsic but also extrinsic curvature as well as torsion in the coset $S O(6) / S O(5)$ space. The torsion is a two-form coupled to the internal d.o.f. of the coset field space, due to the nonvanishing structure constants of the global symmetry group $\mathcal{G}=S O(6)$. We find that the dynamical exchange between the PNGBs and gauge fields is interpreted by a generalized Maurer-Cartan equation sourced by the matter in the SM or beyond, as a fundamental equation in the (EW) effective field theory. The torsion contributes to the non-Abelian dynamics of the PNGBs, which plays a significant role in understanding the nonlinear dynamical (EW) symmetry breaking mechanism.

\section{Curvature of $\mathrm{SO}(6) / \mathrm{SO}(5)$ coset space}

In the $\left(h_{4}, h_{5}\right)$ basis,

$$
\varphi_{\hat{a}} \equiv \frac{h_{\hat{a}}}{h} s_{h}, \quad h=\sqrt{h_{\hat{a}} h_{\hat{a}}} \in S O(5),
$$

with $\hat{a}=1,2,3,4,5$ and $s_{h} \equiv \sin (h / f)$. Then the scalar field becomes

$$
\Phi=\sin \left(\frac{h}{f}\right)\left(\frac{h_{1}}{h}, \frac{h_{2}}{h}, \frac{h_{3}}{h}, \frac{h_{4}}{h}, \frac{h_{5}}{h}, \cot \frac{h}{f}\right)^{T},
$$

where $h=\sqrt{h_{\hat{a}} h_{\hat{a}}}$ is $S O(5)$ invariant if $h^{2}=f^{2}$ or $\hat{h}=1$. The $\Phi$ is $S O(6)$ invariant since $\Phi^{T} \Phi=1$. In the absence of the $\mathrm{SM} \mathrm{GBs,} \mathrm{i.e.,} h_{a}=0$, it just recovers 


$$
\Phi=\left(0,0,0, \frac{h_{4} s_{h}}{\sqrt{h_{4}^{2}+h_{5}^{2}}}, \frac{h_{5} s_{h}}{\sqrt{h_{4}^{2}+h_{5}^{2}}}, c_{h}\right)^{T}
$$

where $h=\sqrt{h_{4}^{2}+h_{5}^{2}}$. Then, the metric of the fundamental scalar field space is $[39,58]$

$$
\begin{aligned}
g_{\hat{a} \hat{b}}(h) & \equiv \frac{\partial \Phi}{\partial h^{\hat{a}}} \cdot \frac{\partial \Phi}{\partial h^{\hat{b}}} \\
& =\frac{1}{f^{2}}\left[\frac{f^{2}}{h^{2}} s_{h}^{2} \delta_{\hat{a} \hat{b}}+\frac{h_{\hat{a}} h_{\hat{b}}}{h^{2}}\left(1-\frac{f^{2}}{h^{2}} s_{h}^{2}\right)\right],
\end{aligned}
$$

where $\hat{a}=1, \ldots, 5$. In the second line, the first terms are the diagonal, while the second terms include nondiagonal ones. In the strong couplings limit, the metric just becomes flat, i.e., $\delta_{\hat{a} \hat{b}}$, in the $f \rightarrow \infty$ limit. In the $(\phi, \psi)$ basis, when SM GBs are present, $\varphi_{a} \sim h_{a} \neq 0$ with $a=1,2,3$, the $S O(6)$ invariant scalar can be parametrized as

$\Phi=s_{h}\left(\frac{h_{1}}{h}, \frac{h_{2}}{h}, \frac{h_{3}}{h}, \frac{\sqrt{h_{4}^{2}+h_{5}^{2}}}{h} c_{\psi}, \frac{\sqrt{h_{4}^{2}+h_{5}^{2}}}{h} s_{\psi}, \cot \frac{h}{f}\right)^{T}$,

where $h=\sqrt{h_{\hat{a}} h_{\hat{a}}}$ is $S O(5)$ invariant if $h^{2}=f^{2}$ or $\hat{h}=1$. When the GBs are vanishing, i.e., $h_{a}=0$, it just recovers that in Eq. (2.63) in the $(\phi, \psi)$ basis. Then, the metric of the scalar field space manifold is

$g_{\hat{a} \hat{b}}=\frac{1}{f^{2}}\left[\frac{f^{2}}{h^{2}} s_{h}^{2} \delta_{\alpha \beta}+\frac{\phi^{2}}{h^{2}} s_{h}^{2} \delta_{\psi \psi}+\frac{h_{\alpha} h_{\beta}}{h^{2}}\left(1-\frac{f^{2}}{h^{2}} s_{h}^{2}\right)\right]$,

where $\alpha=(a, \phi), \beta=(b, \phi)$ and $h_{\phi} \equiv \phi$.

In the $(h, s)$ basis, when the SM GBs are restored, i.e., $\varphi_{a} \equiv h_{a} \neq 0$ with $a=1,2,3$, the scalar becomes

$$
\Phi=\frac{1}{f}\left(h_{1}, h_{2}, h_{3}, h_{4}, h_{5}, \sqrt{f^{2}-h_{\hat{a}} h_{\hat{a}}}\right),
$$

with $\hat{a}=(a, 4,5)$ and $h_{4} \equiv h$ and $h_{5} \equiv s$. In the absence of the GBs, i.e., $h_{a}=0$, it just recovers that in Eq. (2.63) in $h-s$ basis. Then, the metric of the scalar field space is

$$
g_{\hat{a} \hat{b}}(h)=\frac{1}{f^{2}}\left(\delta_{\hat{a} \hat{b}}+\frac{h_{\hat{a}} h_{\hat{b}}}{f^{2}-h_{\hat{c}} h_{\hat{c}}}\right),
$$

where $\hat{a}=1, \ldots, 5$. The metric is the nonlinear transformation of the $S O(5)$ invariant metric on the GB coset space $S O(6) / S O(5) \simeq S^{5}$. This metric is more convenient for our purpose of studying the intrinsic curvature of the scalar space manifold. The determinant and inverse of the metric are

$$
\begin{gathered}
g(h) \equiv \operatorname{det}\left[g_{\hat{a} \hat{b}}(h)\right]=\frac{1}{f^{2 \times 5}}\left(\frac{f^{2}}{f^{2}-h_{\hat{a}} h_{\hat{a}}}\right), \\
g_{\hat{a} \hat{b}}^{-1}(h) \equiv g^{\hat{a} \hat{b}}(h)=f^{2}\left(\delta^{\hat{a} \hat{b}}-\frac{h^{\hat{a}} h^{\hat{b}}}{f^{2}}\right) .
\end{gathered}
$$

Once the metric is obtained, the kinetic terms of the scalars can be expressed as [39,58-60]

$$
\mathcal{L}=\frac{1}{2} g_{\hat{a} \hat{b}} \partial_{\mu} \Phi^{\hat{a}} \partial^{\mu} \Phi^{\hat{b}}
$$

where $\hat{a}, \hat{b}=1, \ldots, 5$. The Lagrangian is manifestly invariant under an $S O(5)$ of linearly realized isospin transformation $h_{\hat{a}}$, by eliminating the $h_{6}$ in the linear $S O(6)$ transformation. The triplet $\varphi_{a}=h_{a}$ with $a=1,2,3$ are identified as the d.o.f. of SM Goldstone bosons. Taking the last metric above as an example, the nonvanishing LeviCivita connection or Christoffel symboles are

$$
\begin{aligned}
\Gamma_{\hat{b} \hat{c}}^{\hat{a}} & =\frac{1}{2} g^{\hat{a} \hat{d}}\left(\partial_{\hat{b}} g_{\hat{c} \hat{d}}+\partial_{\hat{c}} g_{\hat{b} \hat{d}}-\partial_{\hat{d}} g_{\hat{b} \hat{c}}\right)=h^{\hat{a}} g_{\hat{b} \hat{c}}(h) \\
& =\frac{1}{f^{2}}\left(h^{\hat{a}} \delta_{\hat{b} \hat{c}}+\frac{h^{\hat{a}} h_{\hat{b}} h_{\hat{c}}}{f^{2}-h_{\hat{a}} h_{\hat{a}}}\right) .
\end{aligned}
$$

To be explicit, in the Christoffel symbols of the second kind,

$$
\begin{aligned}
\Gamma_{\hat{b} \hat{c}}^{\hat{a}} & =\frac{h^{\hat{a}} h_{\hat{b}} h_{\hat{c}}}{f^{2}\left(f^{2}-h^{2}\right)}, \quad \hat{b} \neq \hat{c}, \\
\Gamma_{\hat{b} \hat{b}}^{\hat{a}} & =\frac{h^{\hat{a}}\left(f^{2}-h^{2}+h_{\hat{b}}^{2}\right)}{f^{2}\left(f^{2}-h^{2}\right)}=\frac{h^{\hat{a}}}{f^{2}}+\frac{h^{\hat{a}} h_{\hat{b}}^{2}}{f^{2}\left(f^{2}-h^{2}\right)} .
\end{aligned}
$$

The Riemann curvature are

$$
\begin{aligned}
& R_{\hat{a} \hat{b} \hat{c} \hat{d}}=g_{\hat{a} \hat{e}}\left(\partial_{\hat{c}} \Gamma^{\hat{e}} \hat{d} \hat{b}-\partial_{\hat{d}} \Gamma_{\hat{c} \hat{b}}^{\hat{e}}+\Gamma^{\hat{f}} \hat{d} \hat{b} \Gamma^{\hat{e}}{ }_{\hat{c} \hat{f}}-\Gamma^{\hat{f}}{ }_{\hat{c} \hat{b}} \Gamma^{\hat{e}} \hat{d} \hat{f}\right) \\
& =\frac{1}{f^{2}}\left(g_{\hat{a} \hat{c}} g_{\hat{b} \hat{d}}-g_{\hat{a} \hat{d}} g_{\hat{b} \hat{c}}\right) \\
& =\frac{1}{f^{2}}\left(\delta_{\hat{a} \hat{c}} \delta_{\hat{b} \hat{d}}+\frac{\delta_{\hat{a} \hat{c}} h_{\hat{b}} h_{\hat{d}}+\delta_{\hat{b} \hat{d}} h_{\hat{a}} h_{\hat{c}}}{f^{2}-h_{\hat{e}} h_{\hat{e}}}-(\hat{c} \leftrightarrow \hat{d})\right) .
\end{aligned}
$$

The Ricci tensors are

$$
\begin{aligned}
& R_{\hat{a} \hat{b}}=\frac{4 h_{\hat{a}} h_{\hat{b}}}{f^{2}\left(f^{2}-h^{2}\right)}=\frac{4}{f^{2}} g_{\hat{a} \hat{b}}, \quad \hat{a} \neq \hat{b}, \\
& R_{\hat{b} \hat{b}}=\frac{4\left(f^{2}-h^{2}+h_{\hat{b}}^{2}\right)}{f^{2}\left(f^{2}-h^{2}\right)}=\frac{4}{f^{2}}+\frac{4 h_{\hat{b}}^{2}}{f^{2}\left(f^{2}-h^{2}\right)} .
\end{aligned}
$$

The extrinsic curvature of the manifold with normal vector $n_{\hat{c}}$ along $\hat{c}$ direction is 


$$
K_{\hat{b}}^{\hat{a}}=\frac{h_{\hat{c}}}{\sqrt{f^{2}-h_{\hat{c}}^{2}}} \delta_{\hat{b}}^{\hat{a}}
$$

Thus, the extrinsic curvature is flat along the arbitrary $\hat{c}$ direction. The intrinsic Ricci curvature of the GBs scalar coset time is $\mathcal{G} / \mathcal{H}=S O(6) / S O(5) \simeq S^{5}$

$$
R=\frac{20}{f^{2}}>0,
$$

where the number $20=5 \times 4$ takes account of the degrees of freedom of the five independent scalars. This positive sign of $R$ indicates that the scalar under the symmetry of compact group $S O(6)$ is in a curved spacetime in analogy to the de Sitter spacetime with a positive cosmological constant. The results in Eqs. (5.72) and (5.74) just recover the specific values of the more general result for the $S O(N+1) / S O(N)$ case in Ref. [59], which gives $R_{\hat{a} \hat{b}}=$ $\left(N_{\varphi}-1\right) / f^{2} g_{\hat{a} \hat{b}}$ and $R=N_{\varphi}\left(N_{\varphi}-1\right) / f^{2}$, where $N_{\varphi}=$ $\operatorname{dim}(\mathcal{G} / \mathcal{H})=N$ is the number of broken generators.

\section{Torsion of $\mathrm{SO}(6) / \mathrm{SO}(5)$ coset space}

It is worth noticing that

$$
\begin{aligned}
\operatorname{Tr}\left(\omega_{\mu} \omega^{\mu}\right) & =\operatorname{Tr}\left[\left(d_{\mu}+e_{\mu}\right)\left(d^{\mu}+e^{\mu}\right)\right]=\operatorname{Tr}\left(d_{\mu} d^{\mu}+e_{\mu} e^{\mu}\right) \\
& =-\operatorname{Tr}\left(\Omega^{\dagger} \partial_{\mu} \Omega \Omega^{\dagger} \partial^{\mu} \Omega\right)=\operatorname{Tr}\left(\partial_{\mu} \Omega^{\dagger} \partial^{\mu} \Omega\right) \\
& =\operatorname{Tr}\left(\partial_{\hat{a}} \Omega^{\dagger} \partial_{\hat{b}} \Omega\right) \partial_{\mu} \phi^{\hat{a}} \partial^{\mu} \phi^{\hat{b}} \\
& \equiv g_{\hat{a} \hat{b}}(\phi) \partial_{\mu} \phi^{\hat{a}} \partial^{\mu} \phi^{\hat{b}},
\end{aligned}
$$

where we have used the property that $\Omega$ is a unitary matrix and $\partial^{\mu} \Omega=\partial^{\mu} \phi^{a} \partial_{a} \Omega(\phi)$ where $\phi^{\hat{a}}$ is an $S O(6)$ invariant scalar with $\hat{a}=1, \ldots 5 . g_{\hat{a} \hat{b}}(\phi)$ is the metric on the coset $S O(6) / S O(5)=S^{5}$ manifold defined as

$$
g_{\hat{a} \hat{b}}(\phi) \equiv \operatorname{Tr}\left(\partial_{\hat{a}} \Omega^{\dagger} \partial_{\hat{b}} \Omega\right) .
$$

To be explicit, one has

$g_{\hat{a} \hat{b}}(\phi)=\frac{4}{f^{2}}\left(\frac{\delta_{\hat{a} \hat{b}}-\frac{\phi_{\hat{a}} \phi_{\hat{b}}}{|\phi|^{2}}}{1+\sqrt{1-\frac{|\phi|^{2}}{f^{2}}}}+\frac{1}{2} \frac{f^{2}}{f^{2}-|\phi|^{2}} \frac{\phi_{\hat{a}} \phi_{\hat{b}}}{|\phi|^{2}}\right)$.

From the definition of the metric in Eq. (5.76), one can define the vielbeins of the coset space as

$$
E_{\hat{a}} \equiv E_{\hat{a}}^{A} T^{A}=-i \Omega^{\dagger} \partial_{\hat{a}} \Omega,
$$

where $T^{A}=\left\{T^{a}, T^{\hat{a}}\right\}$ are the generators of $S O(6)$ as defined in Appendix A 1.

The nonvanishing vielbeins $E_{\hat{a}}^{A}$ those are related to $h(x)$ and $s(x)$ turns out to be

$$
\begin{aligned}
& E_{\hat{a}=4}^{\hat{\alpha}=4}=-\frac{\sqrt{2} s}{f^{2}} \frac{1}{1-\sqrt{1-\frac{h^{2}+s^{2}}{f^{2}}}}, \\
& E_{\hat{a}=5}^{\hat{\alpha}=4}=\frac{\sqrt{2} h}{f^{2}} \frac{1}{1-\sqrt{1-\frac{h^{2}+s^{2}}{f^{2}}}}, \\
& E_{\hat{a}=4}^{\hat{a}=4}=\frac{\sqrt{2}}{f} \frac{s^{2}+\frac{h^{2}}{\sqrt{1-\frac{h^{2}+s^{2}}{f^{2}}}}}{h^{2}+s^{2}}, \quad E_{\hat{a}=5}^{\hat{a}=5}=\frac{\sqrt{2}}{f} \frac{h^{2}+\frac{s^{2}}{\sqrt{1-\frac{h^{2}+s^{2}}{f^{2}}}}}{h^{2}+s^{2}}, \\
& E_{\hat{a}=4}^{\hat{a}=5}=E_{\hat{a}=5}^{\hat{a}=4}=-\frac{\sqrt{2} h s}{f} \frac{1}{h^{2}+s^{2}-f^{2}\left(1+\sqrt{1-\frac{h^{2}+s^{2}}{f^{2}}}\right)} .
\end{aligned}
$$

Thus, on can check that the bilinear form of the vielbein consists of the metric for the coset manifold as $\operatorname{Tr}\left(E_{\hat{a} \hat{b}}\right)=\operatorname{Tr}\left(E_{\hat{a}} E_{\hat{b}}\right)=g_{\hat{a} \hat{b}}(\phi)$, where we have defined

$$
\begin{aligned}
E_{\hat{a} \hat{b}} \equiv E_{\hat{a}} E_{\hat{b}} & =\partial_{\hat{a}} \Omega^{\dagger} \partial_{\hat{b}} \Omega=E_{\hat{a}}^{A} E_{\hat{b}}^{A} \\
& =E_{[\hat{a} \hat{b}]}+E_{(\hat{a} \hat{b})}=\partial_{[\hat{a}} \Omega^{\dagger} \partial_{\hat{b}]} \Omega+\partial_{(\hat{a}} \Omega^{\dagger} \partial_{\hat{b})} \Omega \\
& =\frac{1}{2} E_{\hat{a}}^{A} E_{\hat{b}}^{B}\left(i f_{A B C} T^{C}+\left\{T_{A}, T_{B}\right\}\right),
\end{aligned}
$$

where $f_{A B C}$ is the (totally antisymmetric ${ }^{4}$ ) structure constant, and $d_{A B C}$ is the (totally symmetric) group constant, respectively. Thus, $\operatorname{Tr}\left[E_{\hat{a} \hat{b}} T^{C}\right] \equiv E_{\hat{a}}^{A} E_{\hat{b}}^{B} \operatorname{Tr}\left(T^{A} T^{B} T^{C}\right)=$ $E_{\hat{a}}^{A} E_{\hat{b}}^{B}\left(i f_{A B C}+d_{A B C}\right) / 2$. Taking trace of the symmetric part just corresponds to the metric of the NL- $\sigma$ model in Eq. (5.76) as $g_{\hat{a} \hat{b}} \equiv \operatorname{Tr}\left(E_{\hat{a} \hat{b}}\right)=E_{\hat{a}}^{A} E_{\hat{b}}^{B} \operatorname{Tr}\left(\left\{T_{A}, T_{B}\right\}\right) / 2=$ $k E_{\hat{a}}^{A} E_{\hat{b}}^{B} \delta_{A B} / 2$, where we have used Eq. (A2). According to Eq. (5.78), one has

$$
\partial_{[\hat{b}} E_{\hat{a}]}=-i \partial_{[\hat{b}} \Omega^{\dagger} \partial_{\hat{a}]} \Omega=-i E_{[\hat{b} \hat{a}]} .
$$

With the vielbein, one can define the torsion as a totally antisymmetrized product of vielbines as

$$
\begin{aligned}
T_{\hat{a} \hat{b} \hat{c}} & \equiv-i \operatorname{Tr}\left[\left[E_{\hat{a}}, E_{\hat{b}}\right] E_{\hat{c}}\right]=f_{A B C} E_{\hat{a}}^{A} E_{\hat{b}}^{B} E_{\hat{c}}^{C} \\
& =\operatorname{Tr}\left[\Omega^{\dagger} \partial_{[\hat{a}} \Omega \Omega^{\dagger} \partial_{\hat{b}} \Omega \Omega^{\dagger} \partial_{\hat{c}]} \Omega\right],
\end{aligned}
$$

where in the last equality, we have used Eq. (5.88). It is obvious that the torsion tensor is proportional to the structure constant. Since the structure constants satisfy the Jacobi identity as $f_{A[B C} f_{D] E A}=0$, so does the torsion. According to Eq. (5.78), the vielbein field strength $E_{\hat{a}}^{A}=$ $-i \operatorname{Tr}\left[\Omega^{\dagger} \partial_{\hat{a}} \Omega T^{A}\right]$ satisfies the Maurer-Cartan equation as

\footnotetext{
${ }^{4}$ Due to cyclic property of the trace.
} 


$$
\begin{aligned}
\partial_{[\hat{a}} E_{\hat{b}]}^{A} & =-i \operatorname{Tr}\left[\left(\partial_{[\hat{a}} \Omega^{\dagger} \partial_{\hat{b}]} \Omega\right) T^{A}\right]=-i \operatorname{Tr}\left(E_{[\hat{a}} E_{\hat{b}]} T^{A}\right) \\
& =\frac{1}{2} f_{A B C} E_{\hat{a}}^{C} E_{\hat{b}}^{B} .
\end{aligned}
$$

The symmetrized part of the covariant derivative of the vielbein vanishes. The torsion can be reexpressed as

$$
\begin{aligned}
T_{\hat{a} \hat{b} \hat{c}} & =-2 i \operatorname{Tr}\left[E_{[\hat{a}}, E_{\hat{b}]} E_{\hat{c}}\right]=2 \operatorname{Tr}\left[E_{[\hat{a} \hat{b}]} E_{\hat{c}}\right] \\
& =2 \partial_{[\hat{a}} E_{\hat{b}] C} E_{\hat{c}}^{C}=-2 i \operatorname{Tr}\left[E_{[\hat{a} \hat{b}]} T^{C}\right] E_{\hat{c}}^{C} .
\end{aligned}
$$

\section{Generalized Maurer-Cartan equation}

It is worth noticing that the vielbeins of the coset group are related to the Maurer-Cartan one-form in Eq. (B26) as

$$
\omega_{\mu} \equiv-i \Omega^{\dagger} \partial_{\mu} \Omega=E_{\hat{a}} \partial_{\mu} \phi^{\hat{a}} .
$$

Also note that in the vacuum without matter, i.e., in the absence of an external source, i.e., the field strength tensor $\overline{\mathrm{F}}_{\mu \nu}=0, f_{\mu \nu}=0$. Then, the MC one-form $\omega$ satisfies the well-known two-form MC equation as

$$
d \omega+\frac{i}{2}[\omega, \omega]=0,
$$

where $\omega$ is the Cartan connection of $\mathcal{G}$. Note that the MC one-form $\omega$ is related to the vielbeins of the coset manifold through Eq. (5.85) in differential form as

$$
\omega=E_{\hat{a}} d \phi^{\hat{a}} \equiv E_{\hat{a}} \theta^{\hat{a}},
$$

where $E_{\hat{a}}$ is a frame, and $d \phi^{\hat{a}} \equiv \theta^{\hat{a}}$ is a coframe. Assume that the vielbein frame satisfies the Lie algebra in a nonsymmetric coset, as

$$
\left[E_{\hat{a}}, E_{\hat{b}}\right]=i f_{\hat{a} \hat{b}}^{\hat{c}} E_{\hat{c}},
$$

where $f_{\hat{a} \hat{b}} \hat{c}$ are the structure constants of the Lie algebra. Therefore, in the intrinsic group space, it equivalently becomes the Maurer-Cartan equation in coset manifold space as

$$
d \theta^{\hat{a}}-\frac{1}{2} f_{\hat{b} \hat{c}}{ }^{\hat{c}} \theta^{\hat{b}} \wedge \theta^{\hat{c}}=0
$$

where $f_{b c}^{a}$ is the structure constant of the Lie algebra. This means in the global symmetry without explicit symmetry breaking, i.e., $\bar{F}=0$, or equivalently in the $f \rightarrow \infty$ limit, the manifold of the Lie group $\mathcal{G}=S O(6)$ is curvatureless.

In the presence of external sources from the matter, the global symmetry is explicitly breaking. From they dynamical MC equation in Eq. (B70), we obtain a generalized Maurer-Cartan (GMC) equation sourced by matter in the $\mathrm{SM}$ or beyond as

$$
d \theta^{\hat{a}}-\frac{1}{2} f_{\hat{b} \hat{c}}^{\hat{a}} \theta^{\hat{b}} \wedge \theta^{\hat{c}}=\operatorname{Tr}\left(E^{\hat{a}} f\right),
$$

where on the right-hand side of the equation, $E^{\hat{a}}$ is the inverse of the vielbein $E_{\hat{a}}$, and $f=f_{\mu \nu} d x^{\mu} d x^{\nu} / 2$ is the twoform gauge field strength defined in Eq. (B62). The lefthand side of the equation interprets the dynamics of PNGBs in the intrinsic coset space, with nonlinear contributions from the torsion part, while the right-hand side of the equation interprets that of the gauge field. In other words, the GMC gives a dynamical description between the PNGB $\theta^{a}$ and the non-Abelian gauge fields $f_{\mu \nu}=f_{\mu \nu}^{A} T^{A}$. By using Eq. (5.82), the GMC can also be reexpressed as

$$
d \omega^{A}-\frac{1}{2} f_{B C}{ }^{A} \omega^{B} \wedge \omega^{C}=f^{A},
$$

where $\omega^{A} \equiv \operatorname{Tr}\left[\omega T^{A}\right]$ is the one-form MC form.

\section{Higgs function in NMCHM}

For NMCHM, the kinetic terms of the Higgs sector in Eq. (A63) will be

$$
\mathcal{L}=\frac{1}{2} F(h)^{2} g_{a b} \partial_{\mu} \pi^{a} \partial^{\mu} \pi^{b}+\frac{1}{2} \partial_{\mu} h \partial^{\mu} h,
$$

where the Higgs function $F$ will not be that for the SM as in Eq. (A70). For NMCHM, the coset space $S O(6) / S O(5) \simeq S^{5}$, the scalar in Eq. (5.63) becomes

$$
\Phi=f \sin \frac{h}{f}\left(\hat{h}_{1}, \hat{h}_{2}, \hat{h}_{3}, \hat{h}_{4}, \hat{h}_{5}, \cot \frac{h}{f}\right)^{T},
$$

where $h \equiv \sqrt{h_{\hat{a}} h_{\hat{a}}}$ with $\hat{a}=1, \ldots, 5$. The vacuum of $S O(5)$ is $\langle\Phi\rangle=(0,0,0,0,0, f)^{T}$. The kinetic action is

$$
\mathcal{L}=\frac{f^{2}}{2} \sin ^{2}\left(\frac{h}{f}\right) \partial_{\mu} h^{\hat{a}} \partial^{\mu} h^{\hat{a}}+\frac{1}{2} \partial_{\mu} h \partial^{\mu} h .
$$

By setting set $h_{5}=0$, the scalar above recovers those in coset space $S O(5) / S O(4) \simeq S^{4}$ as $[59,60]$,

$$
\Phi=f \sin \frac{h}{f}\left(\hat{h}_{1}, \hat{h}_{2}, \hat{h}_{3}, \hat{h}_{4}, 0, \cot \frac{h}{f}\right)^{T},
$$

where $h \equiv \sqrt{h_{\alpha} h_{\alpha}}$ with $\alpha=1,2,3,4$. The kinetic term becomes

$$
\mathcal{L}=\frac{f^{2}}{2} \sin ^{2}\left(\frac{h}{f}\right) \partial_{\mu} \hat{h}^{\alpha} \partial^{\mu} \hat{h}^{\alpha}+\frac{1}{2} \partial_{\mu} h \partial^{\mu} h .
$$

By comparing with the kinetic terms of Higgs and GBs with those in Eq. (A63), one obtains the identity that 


$$
F(h)^{2}=\frac{f^{2}}{v^{2}} \sin ^{2}\left(\frac{h_{4}}{f}\right),
$$

where $h_{4}=h+v_{4}$ in the $f \gg 1$ limit and $h$ is the quantum fluctuations of Higgs. After EWSB, the scalars obtain vevs

$$
h_{4} \rightarrow \hat{h}_{4}+\left\langle h_{4}\right\rangle, \quad h \rightarrow \hat{h}+\langle h\rangle,
$$

and one obtains

$$
\frac{v_{4}}{f}=\arcsin \left(\frac{v}{f}\right)
$$

where $v_{4}=\left\langle h_{4}\right\rangle$ and $v=v_{h}=\langle h\rangle$. One can reexpress the Higgs function and expand it as

$$
\begin{aligned}
F(h) & =\cos \left(\frac{h}{f}\right)+\frac{f}{v} \sqrt{1-\frac{v^{2}}{f^{2}}} \sin \left(\frac{h}{f}\right) \\
& \approx 1+\frac{h}{v} \sqrt{1-\frac{v^{2}}{f^{2}}}-\frac{h^{2}}{2 f^{2}}-\frac{h^{3}}{6 f^{2} v} \sqrt{1-\frac{v^{2}}{f^{2}}}+\cdots,
\end{aligned}
$$

which in the weak coupling limit $f \rightarrow \infty$, the scalar manifold tends to be flat, the result above just reduces to the SM Higgs function in Eq. (A70). By substituting back into the curvature scalar in Eq. (A67), one just obtains

$$
R=0,
$$

as expected. In contrast, in the presence of a strong dynamical symmetry breaking scale $f$, by substituting the Higgs function in Eq. (5.100) back into the curvature function in Eq. (A67), one obtains the curvature scalar as

$$
R=\frac{12}{f^{2}}>0
$$

The positive sign of the constant curvature indicates that the coset manifold is a sphere $S^{4}=S O(5) / S O(4)$ spacetime with the strong dynamics breaking scale $f$ as the radius of the sphere. In contrast, for the coset manifold in hyperbolic space $\mathbb{\boxplus}^{4}=S O(4,1) / S O(4)$ spacetime, the curvature scalar becomes

$$
R=-\frac{12}{f^{2}}<0,
$$

which is a constant negative curvature set by strong dynamical symmetry breaking scale $f$ and the corresponding Higgs function is of the form

$$
\begin{aligned}
F(h) & =\cosh \left(\frac{h}{f}\right)+\frac{f}{v} \sqrt{1+\frac{v^{2}}{f^{2}}} \sinh \left(\frac{h}{f}\right) \\
& \approx 1+\frac{h}{v} \sqrt{1+\frac{v^{2}}{f^{2}}}+\frac{h^{2}}{2 f^{2}}+\frac{h^{3}}{6 f^{2} v} \sqrt{1+\frac{v^{2}}{f^{2}}}+\cdots
\end{aligned}
$$

In summary, matching the high-energy effective Lagrangian of the model to the low-energy effective EW chiral Lagrangian, leads to the Higgs functions in $\mathcal{L}$, encoding the information of Higgs nonlinearity.

It noteworthy that the Ricci scalar $R$ can be negative, if the global symmetry group is a noncompact one, but the subgroup to be gauged is a compact one, e.g., if the scalar manifold is a hyperbolic space with constant negative curvature [59]. For our case, this means that the $S O(6)$ global symmetry can be changed into a noncompact group, e.g., $S O(1,5)$, then after gauging the unbroken $S O(5)$, the coset manifold is a five-dimensional hyperbolic space, i.e., $\llbracket^{5}=S O(1,5) / S O(5)$. Equivalently, one may obtain the corresponding effective Lagrangian for noncompact global symmetry by choosing an imaginary decay constant $f \rightarrow$ if [64]. In the future, it would also be interesting to consider the case of strongly coupled gauge field theory, e.g., a four-dimensional conformal field theory $\mathrm{CFT}_{4}$ with a noncompact group manifold $S O(2,4)$, which is isomorphic to a five-dimensional anti-de Sitter (AdS) space, i.e., $\mathrm{AdS}_{5}=S O(2,4) / S O(1,4)$, a space with constant negative curvature.

\section{CONNECTION TO PHYSICAL OBSERVABLES}

In this section, in order to comment our results to the physical observables, we adopt to the following procedure:

First, we need to change the GB matrix in Eq. (4.1) in the $\Omega$ parametrization to those in the $\boldsymbol{\Sigma}$ parametrization. This can be realized by making the shift $f \rightarrow f / 2$ (or $\xi \rightarrow 4 \xi$ ) and $\psi \rightarrow \psi / 2$ as Eq. (2.25).

Second, for the NMCHM, since the kinetic terms of $h, s$ are not canonically normalized at the stage, we need to make eigenstate basis transformation as in Eq. (5.26), in order to obtain the canonically normalized kinetic terms of Higgs $h$ and $s$.

Third, in order to relate our results to the physical observables below the $\mathrm{TeV}$ scale, we need to consider the EW vacuum expectation value $v$ after EWSB. Therefore, we make $h \rightarrow v+\sqrt{1-\xi} h$ and $s \rightarrow s$, and expand up to second order of $\xi$.

\section{A. Phenomenology of scalar sector}

In addition to the Higgs boson $h \sim h_{4}$ itself, the new scalar $s \sim h_{5}$ is also a PNGB singlet with no EW charge, which could be light. At low energy, the phenomenology of 
a scalar sector of the model is very similar to that of the singlet extended standard model. The singlet scalar $s$ could be a dark matter candidate, if it satisfies a $Z_{2}$ symmetry in the Lagrangian below.

\section{Higgs kinetics and self-interactions}

The kinetic terms of the $h$ and $s$, as well as the mass term of the gauge boson, can be obtained from $p^{2}$ order Lagrangian in Eq. (5.9). These kinetic terms are consistent with Eq. (11) in Ref. [23] or Eq. (A.5) in Ref. [24] for NMCHM as shown in Eq. (5.10). After EWSB, by transforming $(h, s)$ into the canonically normalized eigenstate with the aid of Eq. (5.26), one obtains

$$
\begin{aligned}
\mathcal{L}_{\text {eign }}= & \frac{1}{2}\left(\partial_{\mu} h\right)\left(\partial^{\mu} h\right)\left(1+2 a_{h h} \frac{h}{v}+b_{h h} \frac{h^{2}}{v^{2}}+b_{h s} \frac{s^{2}}{v^{2}}+\cdots\right) \\
& +\frac{1}{2}\left(\partial_{\mu} s\right)\left(\partial^{\mu} s\right)\left(1+2 a_{s h} \frac{h}{v}+b_{s h} \frac{h^{2}}{v^{2}}+b_{s s} \frac{s^{2}}{v^{2}}+\cdots\right) \\
& +\partial_{\mu} h \partial^{\mu} s\left(c_{s} \frac{s}{v}+d_{h s} \frac{h s}{v^{2}}+\cdots\right)-V_{\text {eff }}(h, s) \\
& +\left(1+2 a_{V h} \frac{h}{v}+b_{V h} \frac{h^{2}}{v^{2}}+b_{V s} \frac{s^{2}}{v^{2}}+b_{V h s} \frac{h s}{v^{2}}+\cdots\right) \\
& \times\left(m_{W}^{2} M_{\mu}^{+} W^{-\mu}+\frac{1}{2} m_{Z}^{2} Z_{\mu} Z^{\mu}\right),
\end{aligned}
$$

where $V_{\text {eff }}(h, s)$ is the effective Higgs potential given in Eq. (6.4), and $\cdots$ denotes higher dimensional terms. From the above expansion, one can read the terms

$$
\begin{aligned}
a_{h h} & =\frac{\xi}{\sqrt{1-\xi}}, \quad b_{h h}=\frac{\xi(1+3 \xi)}{1-\xi}, \quad b_{h s}=\frac{\xi^{2}}{1-\xi} ; \\
a_{s h} & =0, \quad b_{s h}=0, \quad b_{s s}=\frac{\xi}{1-\xi} ; \\
c_{s} & =\frac{\xi}{\sqrt{1-\xi}}, \quad d_{h s}=\frac{\xi(1+\xi)}{1-\xi} ; \\
a_{V h} & =\sqrt{1-\xi}, \quad b_{V h}=1-\xi, \quad b_{V s}=0, \quad b_{V h s}=0,
\end{aligned}
$$

which are consistent with those listed in Table 1 in Ref. [26]. Since $b_{V h s}=0$, the above action has a $Z_{2}$ symmetry for the singlet scalar $s$.

\section{Higgs potential}

Once an effective Higgs portential $V_{\text {eff }}(h, s)$ is dynamically generated, e.g., through the Coleman-Weinberg effective potential approach, the Higgs potential can be parametrized as

$V_{\mathrm{eff}}(h, s)=\frac{\mu_{h}^{2}}{2} h^{2}+\frac{\lambda_{h}}{4} h^{4}+\frac{\mu_{s}^{2}}{2} s^{2}+\frac{\lambda_{s}}{4} s^{4}+\frac{\lambda}{2} h^{2} s^{2}$.
By making shift $(h, s) \rightarrow f(h, s)$ so that the $(h, s)$ are dimensionless, the parametrization just recovers Eq. (A.8) in Ref. [24] where $\eta \equiv s$. After transforming to eigenstates, by using $h \rightarrow v+\sqrt{1-\xi} h$ and $s \rightarrow s$, one has

$$
\begin{aligned}
V_{\text {eff }}= & \frac{m_{h}^{2}}{2} h^{2}+\frac{\lambda_{h^{3}}}{2} h^{3}+\frac{\lambda_{h^{4}}}{4} h^{4}+\frac{m_{s}^{2}}{2} s^{2} \\
& +\frac{\lambda h s^{2}}{2} h s^{2}+\frac{\lambda_{s^{4}}}{4} s^{4}+\frac{\lambda_{h^{2} s^{2}}}{2} h^{2} s^{2},
\end{aligned}
$$

where

$m_{h}^{2}=\left(\mu_{h}^{2}+3 v^{2} \lambda_{h}\right)(1-\xi)=2 v^{2} \lambda_{h}(1-\xi), \quad m_{s}^{2}=\mu_{s}^{2}+\lambda v^{2}$, $\lambda_{h^{3}}=2 v \lambda_{h}(1-\xi)^{3 / 2}, \quad \lambda_{h^{4}}=\lambda_{h}(1-\xi)^{2}, \quad \lambda_{s^{4}}=\lambda_{s}$,

$\lambda_{h s^{2}}=2 v \lambda \sqrt{1-\xi}, \quad \lambda_{h^{2} s^{2}}=\lambda(1-\xi)$,

where we have used that $\mu_{h}^{2}=-v \lambda_{h}$ from the minimization condition in Eq. (6.9). The PNGB interactions between $(h, s)$ just recover the Feynman rules in Table 2 in Ref. [24]. By using the model-dependent relations as those in Eq. (2.11), the effective Higgs portal in the NMCHM up to quartic order can be parametrized as [26]

$$
\begin{aligned}
& V_{\mathrm{eff}}=-\gamma \cos ^{2}\left(\frac{\psi}{f}\right) \sin ^{2}\left(\frac{\phi}{f}\right)+\beta \cos ^{4}\left(\frac{\psi}{f}\right) \sin ^{4}\left(\frac{\phi}{f}\right) \\
&+\delta \sin ^{2}\left(\frac{\phi}{f}\right)+\sigma \cos ^{2}\left(\frac{\psi}{f}\right) \sin ^{4}\left(\frac{\phi}{f}\right)+\chi \sin ^{4}\left(\frac{\phi}{f}\right) \\
& \stackrel{\psi=0}{=}-\bar{\gamma} \sin ^{2}\left(\frac{\phi}{f}\right)+\bar{\beta} \sin ^{4}\left(\frac{\phi}{f}\right),
\end{aligned}
$$

where $\bar{\gamma} \equiv \gamma-\delta$, and $\bar{\beta} \equiv \beta+\sigma+\chi$ with

$\gamma=-\frac{f^{2}}{2}\left(\mu_{h}^{2}-\mu_{s}^{2}\right), \quad \beta=\frac{f^{4}}{4}\left(\lambda_{h}+\lambda_{s}-2 \lambda\right)$,

$\delta=\frac{f^{2}}{2} \mu_{s}^{2}, \quad \sigma=\frac{f^{4}}{2}\left(\lambda-\lambda_{s}\right), \quad \chi=\frac{f^{4}}{4} \lambda_{s}$.

The parameters can also reexpress the free parameters of the Higgs potential as

$$
\begin{aligned}
& \mu_{h}^{2}=-\frac{2}{f^{2}} \bar{\gamma}, \quad \mu_{s}^{2}=\frac{2 \delta}{f^{2}}, \\
& \lambda_{h}=\frac{4 \bar{\beta}}{f^{4}}, \quad \lambda=\frac{2(\sigma+2 \chi)}{f^{4}}, \quad \lambda_{s}=\frac{4 \chi}{f^{4}} .
\end{aligned}
$$

By minimizing the Higgs potential after EWSB, one obtains an EW vev,

$$
v^{2}=-\frac{\mu_{h}^{2}}{\lambda_{h}}, \quad \xi=\frac{\bar{\gamma}}{2 \bar{\beta}} .
$$

Thus, by making the transformation to the mass eigenstate, one can read the physical Higgs $h$ and the singlet $s$ mass as 


$$
\begin{aligned}
& m_{h}^{2}=2 \lambda_{h} v^{2}(1-\xi)=\frac{8 \bar{\beta}}{v^{2}}(1-\xi) \xi^{2}, \\
& m_{s}^{2}=\mu_{s}^{2}+\lambda v^{2}=\frac{2}{v^{2}}[\delta+(\sigma+2 \chi) \xi] \xi
\end{aligned}
$$

and there is no mixing term, i.e., $m_{h s}=0$. The results recover the physical masses of PNGB in Eq. (A.9) in Ref. [24].

\section{B. Low-energy EWPT oblique parameters}

The Wilson coefficients $c_{n}$ (or $c_{\tilde{n}}$ for the $C P$-odd case) in the low-energy effective chiral Lagrangian are related to the most significant EW oblique parameters from EWPT due to two-point functions, i.e., the $S$ and $T$ parameter [65], which parametrize new physics contributions to electroweak radiative corrections. For example, the EW oblique parameters $S$ and $T$ are related to $c_{1}$ and $c_{T}$, respectively, as $[66,67]$

$$
\alpha_{e m} \Delta S=-8 e^{2} c_{1}, \quad \alpha_{e m} \Delta T=2 c_{T},
$$

where $\alpha_{e m}=e^{2} /(4 \pi)$. In the NMCHM, we have

$$
c_{1}=\xi \bar{c}_{1}, \quad c_{T}=-\frac{\xi}{4} \bar{c}_{T}
$$

at tree level. The $\mathrm{S}$ parameter obtains corrections from the operator at $p^{4}$ order, and the $T$ parameter obtains corrections from the custodial breaking operator at $p^{2}$ order. $^{5}$

\section{Triple anomalous gauge-boson couplings}

\section{CP-even case}

The triple gauge-boson couplings in the pure weak boson sector $[47,68,69]$ can be parametrized as

$$
\begin{aligned}
\mathcal{L}_{\text {eff, } C P}^{W W V}= & -i g_{W W V}\left[\kappa_{V} W_{\mu}^{+} W_{\nu}^{-} V^{\mu \nu}\right. \\
& +g_{1}^{V}\left(W_{\mu \nu}^{+} W^{-\mu} V^{\nu}-W_{\mu}^{+} V_{\nu} W^{-\mu \nu}\right) \\
& -i g_{5}^{V} \epsilon^{\mu \nu \rho \sigma}\left(W_{\mu}^{+} \partial_{\rho} W_{\nu}^{-}-W_{\nu}^{-} \partial_{\rho} W_{\mu}^{+}\right) V_{\sigma} \\
& \left.+g_{6}^{V}\left(\partial_{\mu} W^{+\mu} W^{-\nu}-\partial_{\mu} W^{-\mu} W^{+\nu}\right) V_{\nu}\right],
\end{aligned}
$$

where $V \equiv\{\gamma, Z\}$ and

$$
g_{W W \gamma}=g s_{\theta} \equiv e, \quad g_{W W Z}=g c_{\theta},
$$

\footnotetext{
${ }^{5}$ Note that, in the presence of heavy resonances, either scalar, vector bosons, or heavy fermions at the one-loop level will contribute radiative corrections to the oblique $T$ parameter, too. By integrating out these heavy resonances, the oblique $T$ parameter may also obtain nonvanishing correction from effective operators at $p^{4}$ order.
}

where $e$ is the electric charge. The chiral Lagrangian contributes to the anomalous cubic couplings as [69]

$$
\begin{aligned}
& \Delta \kappa_{\gamma}=-2 \frac{e^{2}}{s_{\theta}^{2}}\left(\bar{c}_{1}-\bar{c}_{2}-\bar{c}_{3}\right) \xi, \\
& \Delta \kappa_{Z}=2 e^{2}\left(\frac{2}{c_{2 \theta}} \bar{c}_{1}-\frac{1}{c_{\theta}^{2}} \bar{c}_{2}+\frac{1}{s_{\theta}^{2}} \bar{c}_{3}\right) \xi, \\
& \Delta g_{1}^{Z}=\frac{2 e^{2}}{s_{\theta}^{2}} \frac{1}{c_{\theta}^{2}}\left(\frac{s_{\theta}^{2}}{c_{2 \theta}} \bar{c}_{1}+\bar{c}_{3}\right) \xi, \\
& \Delta g_{5}^{Z}=0, \\
& \Delta g_{6}^{\gamma}=-4 \frac{e^{2}}{s_{\theta}^{2}} \bar{c}_{6} \xi, \\
& \Delta g_{6}^{Z}=4 \frac{e^{2}}{c_{\theta}^{2}} \bar{c}_{6} \xi .
\end{aligned}
$$

It is obvious that, from the high-energy view point, the anomalous triple gauge couplings of the SM comes from $\overline{\mathcal{L}}_{1,2,3,6}$ for NMCHM.

\section{CP-odd case}

The $C P$-odd triple gauge boson couplings can be parametrized as $[48,70]$

$$
\begin{aligned}
\mathcal{L}_{\text {eff }, \ell P}^{W W V}= & g_{W W V}\left[-i \tilde{\kappa}_{V} W_{\mu}^{\dagger} W_{\nu} \tilde{V}^{\mu \nu}\right. \\
& +g_{4}^{V} W_{\mu}^{\dagger} W_{\nu}\left(\partial^{\mu} V^{\nu}+\partial^{\nu} V^{\mu}\right) \\
& +\tilde{g}_{6}^{V}\left(W_{\nu}^{\dagger} \partial_{\mu} W^{\mu}+W_{\nu} \partial_{\mu} W^{\dagger \mu}\right) V^{\nu} \\
& \left.+\tilde{g}_{7}^{V} W_{\mu}^{\dagger} W^{\mu} \partial^{\nu} V_{\nu}\right], \\
\mathcal{L}_{\text {eff }, \ell P}^{Z Z Z}= & \tilde{g}_{3 Z} Z_{\mu} Z^{\mu} \partial_{\nu} Z^{\nu},
\end{aligned}
$$

where [48]

$$
\begin{array}{rlrl}
\Delta \tilde{\kappa}_{\gamma} & =-\frac{2 e^{2}}{s_{\theta}^{2}} \bar{c}_{\tilde{1}} \xi, \quad \Delta \tilde{\kappa}_{Z}=\frac{2 e^{2}}{c_{\theta}^{2}} \bar{c}_{\tilde{1}} \xi, \\
\Delta g_{4}^{\gamma} & =0, \quad \Delta g_{4}^{Z}=0, \\
\Delta \tilde{g}_{6}^{\gamma} & =0, \quad \Delta \tilde{g}_{6}^{Z}=0, \\
\Delta \tilde{g}_{7}^{\gamma} & =0, & \Delta \tilde{g}_{7}^{Z}=0, \\
\Delta \tilde{g}_{3 Z} & =0 .
\end{array}
$$

It is obvious that, from the high-energy viewpoint, the $C P$-violating anomalous triple gauge couplings come only from $\overline{\mathcal{L}}_{\tilde{1}}$ for NMCHM.

\section{Quartic anomalous gauge-boson couplings}

The effective Lagrangian of quartic gauge bosons can be parametrized as $[47,69]$ 


$$
\begin{aligned}
\mathcal{L}_{\mathrm{eff}}^{V^{4}}= & g^{2}\left[g_{Z Z}^{(1)}\left(Z_{\mu} Z^{\mu}\right)^{2}+g_{W W}^{(1)} W_{\mu}^{+} W^{+\mu} W_{\nu}^{-} W^{-\nu}\right. \\
& -g_{W W}^{(2)}\left(W_{\mu}^{+} W^{-\mu}\right)^{2} \\
& +g_{V V^{\prime}}^{(3)} W^{+\mu} W^{-\nu}\left(V_{\mu} V_{\nu}^{\prime}+V_{\mu}^{\prime} V_{\nu}\right) \\
& \left.-g_{V V^{\prime}}^{(4)} W_{\mu}^{+} W^{-\mu} V^{\nu} V_{\nu}^{\prime}+i g_{V V^{\prime}}^{(5)} e^{\mu \nu \rho \sigma} W_{\mu}^{+} W_{\nu}^{-} V_{\rho} V_{\sigma}^{\prime}\right],
\end{aligned}
$$

where nonvanishing couplings at the tree level in the SM are

$$
\begin{array}{rlrl}
g_{W W}^{(1) S M} & =\frac{1}{2}, & & g_{W W}^{(2) S M}=\frac{1}{2}, \\
g_{Z Z}^{(3) S M}=\frac{c_{\theta}^{2}}{2}, & g_{\gamma \gamma}^{(3) S M}=\frac{s_{\theta}^{2}}{2} \\
g_{Z \gamma}^{(3) S M}=\frac{s_{2 \theta}}{2}, & g_{Z Z}^{(4) S M}=c_{\theta}^{2}, \\
g_{\gamma \gamma}^{(4) S M}=s_{\theta}^{2}, & g_{Z \gamma}^{(4) S M}=s_{2 \theta} .
\end{array}
$$

The chiral Lagrangian contributes to the anomalous quartic couplings as $[47,69]$

$$
\begin{aligned}
\Delta g_{W W}^{(1)}= & \frac{e^{2}}{4 s_{\theta}^{2}}\left[\left[8\left(\frac{s_{\theta}^{2}}{c_{2 \theta}} \bar{c}_{1}+\bar{c}_{3}\right)\right] \xi+32 \bar{c}_{5} \xi^{2}\right], \\
\Delta g_{W W}^{(2)}= & \frac{e^{2}}{4 s_{\theta}^{2}}\left[\left(\frac{8 s_{\theta}^{2}}{c_{2 \theta}} \bar{c}_{1}+8 \bar{c}_{3}+12 \bar{c}_{6}\right) \xi\right. \\
& \left.-8\left(8 \bar{c}_{4}+4 \bar{c}_{5}+\bar{c}_{6}\right) \xi^{2}\right], \\
\Delta g_{Z Z}^{(1)}= & \frac{e^{2}}{4 s_{\theta}^{2}} \frac{1}{c_{\theta}^{4}}\left[-3 \bar{c}_{6} \xi+2\left(8\left(\bar{c}_{4}+\bar{c}_{5}\right)+\bar{c}_{6}\right) \xi^{2}\right], \\
\Delta g_{Z Z}^{(3)}= & \frac{e^{2}}{4 s_{\theta}^{2}} \frac{1}{c_{\theta}^{2}}\left[\left(\frac{2 s_{2 \theta}^{2}}{c_{2 \theta}} \bar{c}_{1}+8 c_{\theta}^{2} \bar{c}_{3}-8 s_{\theta}^{4} \bar{c}_{6}\right) \xi+32 \bar{c}_{5} \xi^{2}\right], \\
\Delta g_{Z Z}^{(4)}= & \frac{e^{2}}{4 s_{\theta}^{2}} \frac{1}{c_{\theta}^{2}}\left[\left[4\left(\frac{s_{2 \theta}^{2}}{c_{2 \theta}} \bar{c}_{1}+4 c_{\theta}^{2} \bar{c}_{3}+3 \bar{c}_{6}\right)\right] \xi\right. \\
& \left.-8\left(8 \bar{c}_{4}+\bar{c}_{6}\right) \xi^{2}\right], \\
\Delta g_{\gamma \gamma}^{(3)}= & \frac{e^{2}}{4 s_{\theta}^{2}} s_{\theta}^{2}\left(-8 \bar{c}_{6}\right) \xi, \\
\Delta g_{\gamma Z}^{(3)}= & \frac{2 e^{2}}{s_{\theta}^{2}} \frac{s_{\theta}}{c_{\theta}}\left(\frac{s_{\theta}^{2}}{c_{2 \theta}} \bar{c}_{1}+2 s_{\theta}^{2} \bar{c}_{6}+\bar{c}_{3}\right) \xi, \\
\Delta g_{\gamma Z}^{(4)}= & \frac{4 e^{2}}{s_{\theta}^{2}} \frac{s_{\theta}}{c_{\theta}}\left(\frac{s_{\theta}^{2}}{c_{2 \theta}} \bar{c}_{1}+\bar{c}_{3}\right) \xi, \\
\Delta g_{\gamma Z}^{(5)}= & 0 .
\end{aligned}
$$

From the high-energy viewpoint, the anomalous triple gauge couplings of the SM comes from $\overline{\mathcal{L}}_{1,2,3,4,5,6}$ for NMCHM.

\section{E. $H V V$ anomalous couplings}

\section{CP-even case}

The anomalous couplings of the Higgs bosons can be parametrized as $[40,47,54,69]$

$$
\begin{aligned}
\mathcal{L}_{\mathrm{eff}, C P}^{\mathrm{HVV}}= & g_{H \gamma \gamma} A_{\mu \nu} A^{\mu \nu} h+g_{H Z \gamma}^{(1)} A_{\mu \nu} Z^{\mu} \partial^{\nu} h+g_{H Z \gamma}^{(2)} A_{\mu \nu} Z^{\mu \nu} h \\
& +g_{H Z Z}^{(1)} Z_{\mu \nu} Z^{\mu} \partial^{\nu} h+g_{H Z Z}^{(2)} Z_{\mu \nu} Z^{\mu \nu} h \\
& +g_{H Z Z}^{(3)} Z_{\mu} Z^{\mu} h+g_{H Z Z}^{(4)} Z_{\mu} Z^{\mu} \square h \\
& +g_{H Z Z}^{(5)} \partial_{\mu} Z^{\mu} Z_{\nu} \partial^{\nu} h+g_{H Z Z}^{(6)}\left(\partial_{\mu} Z^{\mu}\right)^{2} h \\
& +g_{H W W}^{(1)}\left(W_{\mu \nu}^{+} W^{-\mu} \partial^{\nu} h+\text { H.c. }\right)+g_{H W W}^{(2)} W_{\mu \nu}^{+} W^{-\mu \nu} h \\
& +g_{H W W}^{(3)} W_{\mu}^{+} W^{-\mu} h+g_{H W W}^{(4)} W_{\mu}^{+} W^{-\mu} \square h \\
& +g_{H W W}^{(5)}\left(\partial_{\mu} W^{+\mu} W_{\nu}^{-} \partial^{\nu} h+\text { H.c. }\right) \\
& +g_{H W W}^{(6)} \partial_{\mu} W^{+\mu} \partial_{\nu} W^{-\nu} h,
\end{aligned}
$$

where the contribution of the anomalous couplings are separated from that of the SM one as

$$
g_{H V V}^{(i)} \approx g_{H V V}^{(i) S M}+\Delta g_{H V V}^{(i)},
$$

where $V=\left\{\gamma, Z, W_{ \pm}\right\}$. All the tree-level SM couplings above vanish, except the following:

$$
\begin{aligned}
g_{H Z Z}^{(3) S M} & =\frac{e^{2}}{4 v} \frac{m_{Z}^{2}}{e^{2}}\left(-2 c_{H}\right)=-\frac{m_{Z}^{2}}{2 v}, \\
g_{H W W}^{(3) S M} & =\frac{e^{2}}{4 v} \frac{m_{Z}^{2} c_{\theta}^{2}}{e^{2}}\left[-4\left(c_{H}+c_{C}\right)\right]=-2 \frac{m_{W}^{2}}{v} .
\end{aligned}
$$

The anomalous coefficients are related to the coefficients of low-energy effective chiral Lagrangians as $[40,47,69,71]$

$$
\begin{aligned}
\Delta g_{H \gamma \gamma}= & \frac{e^{2}}{4 v}\left[-8\left(\bar{c}_{B \boldsymbol{\Sigma}}+\bar{c}_{W \boldsymbol{\Sigma}}-\bar{c}_{1}\right) \xi\right. \\
& \left.+4\left(\bar{c}_{B \boldsymbol{\Sigma}}+\bar{c}_{W \boldsymbol{\Sigma}}-\bar{c}_{1}\right) \xi^{2}\right], \\
\Delta g_{H Z \gamma}^{(1)}= & \frac{e^{2}}{4 v} \frac{1}{s_{2 \theta}}\left[-32\left(\bar{c}_{2}-\bar{c}_{3}\right) \xi+64\left(\bar{c}_{2}-\bar{c}_{3}\right) \xi^{2}\right], \\
\Delta g_{H Z \gamma}^{(2)}= & \frac{e^{2}}{4 v} \frac{c_{\theta}}{s_{\theta}}\left[\frac{8}{c_{\theta}^{2}}\left(2 s_{\theta}^{2} \bar{c}_{B \boldsymbol{\Sigma}}-2 c_{\theta}^{2} \bar{c}_{W \boldsymbol{\Sigma}}+c_{2 \theta} \bar{c}_{1}\right) \xi\right. \\
& \left.-\frac{4}{c_{\theta}^{2}}\left(2 s_{\theta}^{2} \bar{c}_{B \boldsymbol{\Sigma}}-2 c_{\theta}^{2} \bar{c}_{W \Sigma}+c_{2 \theta} \bar{c}_{1}\right) \xi^{2}\right], \\
\Delta g_{H Z Z}^{(1)}= & \frac{e^{2}}{4 v} \frac{1}{c_{\theta}^{2}}\left[16\left(\bar{c}_{2}+\frac{c_{\theta}^{2}}{s_{\theta}^{2}} \bar{c}_{3}\right) \xi-32\left(\bar{c}_{2}+\frac{c_{\theta}^{2}}{s_{\theta}^{2}} \bar{c}_{3}\right) \xi^{2}\right], \\
\Delta g_{H Z Z}^{(2)}= & -\frac{e^{2}}{4 v} \frac{c_{\theta}^{2}}{s_{\theta}^{2}}\left[8\left(\frac{s_{\theta}^{4}}{c_{\theta}^{4}} \bar{c}_{B \boldsymbol{\Sigma}}+\bar{c}_{W \boldsymbol{\Sigma}}+\frac{s_{\theta}^{2}}{c_{\theta}^{2}} \bar{c}_{1}\right) \xi\right. \\
& \left.-4\left(\frac{s_{\theta}^{4}}{c_{\theta}^{4}} \bar{c}_{B \boldsymbol{\Sigma}}+\bar{c}_{W \mathbf{\Sigma}}+\frac{s_{\theta}^{2}}{c_{\theta}^{2}} \bar{c}_{1}\right) \xi^{2}\right],
\end{aligned}
$$




$$
\begin{aligned}
& \Delta g_{H Z Z}^{(3)}=-\frac{e^{2}}{4 v} \frac{m_{Z}^{2}}{e^{2}} \bar{c}_{T} \xi \\
& \Delta g_{H Z Z}^{(4)}=-\frac{e^{2}}{4 v} \frac{1}{s_{2 \theta}^{2}}\left(-64 \bar{c}_{6} \xi+128 \bar{c}_{6} \xi^{2}\right), \\
& \Delta g_{H Z Z}^{(5)}=-\frac{e^{2}}{4 v} \frac{1}{s_{2 \theta}^{2}}\left(128 \bar{c}_{6} \xi-256 \bar{c}_{6} \xi^{2}\right), \\
& \Delta g_{H Z Z}^{(6)}=-\frac{e^{2}}{4 v} \frac{1}{s_{2 \theta}^{2}}\left(64 \bar{c}_{6} \xi-32 \bar{c}_{6} \xi^{2}\right) \text {, } \\
& \Delta g_{H W W}^{(1)}=\frac{e^{2}}{4 v} \frac{1}{s_{\theta}^{2}}\left(64 \bar{c}_{3} \xi-32 \bar{c}_{3} \xi^{2}\right), \\
& \Delta g_{H W W}^{(2)}=\frac{e^{2}}{4 v} \frac{1}{s_{\theta}^{2}}\left(-16 \bar{c}_{W \Sigma} \xi+8 \bar{c}_{W \Sigma} \xi^{2}\right) \text {, } \\
& \Delta g_{H W W}^{(3)}=\frac{e^{2}}{4 v} \frac{m_{Z}^{2} c_{\theta}^{2}}{e^{2}}\left(\frac{32 e^{2}}{c_{2 \theta}} \bar{c}_{1}-\frac{4 c_{\theta}^{2}}{c_{2 \theta}} \bar{c}_{T}\right) \xi, \\
& \Delta g_{H W W}^{(4)}=-\frac{e^{2}}{4 v} \frac{1}{s_{\theta}^{2}}\left(-32 \bar{c}_{6} \xi+64 \bar{c}_{6} \xi^{2}\right), \\
& \Delta g_{H W W}^{(5)}=-\frac{e^{2}}{4 v} \frac{1}{s_{\theta}^{2}}\left(32 \bar{c}_{6} \xi-64 \bar{c}_{6} \xi^{2}\right) \text {, } \\
& \Delta g_{H W W}^{(6)}=-\frac{e^{2}}{4 v} \frac{1}{s_{\theta}^{2}}\left(32 \bar{c}_{6} \xi-16 \bar{c}_{6} \xi^{2}\right) \text {. }
\end{aligned}
$$

It is obvious that, the $C P$-even anomalous couplings $\Delta g_{H V V}^{(1,2,3)}$ of the $\mathrm{SM}$ comes from operators $\overline{\mathcal{L}}_{B \boldsymbol{\Sigma}, W \boldsymbol{\Sigma}, 1,2,3}$, while the additional $\Delta g_{H V V}^{(4,5,6)}$ all attribute to one operator $\overline{\mathcal{L}}_{6}$ in the high energy.

\section{CP-odd case}

The anomalous couplings of the $C P$-odd interactions involving the Higgs to two gauge bosons can be parametrized as $[48,70]$

$$
\begin{aligned}
\mathcal{L}_{\mathrm{eff}, \ell P}^{\mathrm{HVV}}= & \tilde{g}_{H \gamma \gamma} h A_{\mu \nu} \tilde{A}^{\mu \nu}+\tilde{g}_{H Z \gamma} h A_{\mu \nu} \tilde{Z}^{\mu \nu} \\
& +\tilde{g}_{H Z Z}^{(2)} h Z_{\mu \nu} \tilde{Z}^{\mu \nu}+\tilde{g}_{H W W}^{(2)} h W_{\mu \nu}^{+} \tilde{W}^{-\mu \nu} \\
& +\left[\tilde{g}_{H W W}^{(1)}\left(W_{\mu \nu}^{+} W^{-\mu} \partial^{\nu} h\right)+\text { H.c. }\right] \\
& +\left[\tilde{g}_{H W W}^{(5)}\left(\partial_{\mu} W^{+\mu} W_{\nu}^{-} \partial^{\nu} h\right)+\text { H.c. }\right]
\end{aligned}
$$

where the anomalous $C P$-odd $H V V$ couplings at the tree level are [48]

$$
\begin{aligned}
\tilde{g}_{H \gamma \gamma}= & \frac{4 e^{2}}{v}\left[\frac{1}{2}\left(-2 \bar{c}_{\tilde{B} \Sigma}-\bar{c}_{\tilde{W} \Sigma}+\bar{c}_{\tilde{1}}\right) \xi\right. \\
& \left.+\frac{1}{4}\left(2 \bar{c}_{\tilde{B} \Sigma}+\bar{c}_{\tilde{W} \Sigma}-\bar{c}_{\tilde{1}}-24 \bar{c}_{\tilde{2}}\right) \xi^{2}\right],
\end{aligned}
$$

$$
\begin{aligned}
\tilde{g}_{H Z \gamma}= & -\frac{8 e^{2} s_{\theta}}{v c_{\theta}}\left[-\frac{1}{4 s_{\theta}^{2}}\left(4 s_{\theta}^{2} \bar{c}_{\tilde{B} \boldsymbol{\Sigma}}-2 c_{\theta}^{2} \bar{c}_{\tilde{W} \boldsymbol{\Sigma}}+c_{2 \theta} \bar{c}_{\tilde{1}}\right) \xi\right. \\
& +\frac{1}{8 s_{\theta}^{2}}\left(4 s_{\theta}^{2} \bar{c}_{\tilde{B} \boldsymbol{\Sigma}}-2 c_{\theta}^{2} \bar{c}_{\tilde{W} \boldsymbol{\Sigma}}+c_{2 \theta} \bar{c}_{\tilde{1}}\right. \\
& \left.\left.+8 \bar{c}_{\tilde{2}}\left(6 c_{\theta}^{2}-1\right)\right) \xi^{2}\right], \\
\tilde{g}_{H Z Z}^{(2)}= & \frac{4 e^{2} s_{\theta}^{2}}{v c_{\theta}^{2}}\left[-\frac{1}{2 s_{\theta}^{4}}\left(2 s_{\theta}^{4} \bar{c}_{\tilde{B} \boldsymbol{\Sigma}}+c_{\theta}^{4} \bar{c}_{\tilde{W} \boldsymbol{\Sigma}}+c_{\theta}^{2} s_{\theta}^{2} \bar{c}_{\tilde{1}}\right) \xi\right. \\
& +\frac{1}{4 s_{\theta}^{4}}\left(2 s_{\theta}^{4} \bar{c}_{\tilde{B} \boldsymbol{\Sigma}}+c_{\theta}^{4} \bar{c}_{\tilde{W} \boldsymbol{\Sigma}}+c_{\theta}^{2} s_{\theta}^{2} \bar{c}_{\tilde{1}}\right. \\
& \left.\left.+\left[-8 c_{2 \theta}-3\left(c_{4 \theta}+3\right)\right] \bar{c}_{\tilde{2}}\right) \xi^{2}\right], \\
\tilde{g}_{H W W}^{(1)}= & 0, \\
\tilde{g}_{H W W}^{(2)}= & -\frac{2 e^{2}}{v s_{\theta}^{2}}\left(2 \bar{c}_{\tilde{W} \boldsymbol{\Sigma}} \xi+\left(20 \bar{c}_{\tilde{2}}-\bar{c}_{\tilde{W} \boldsymbol{\Sigma}}\right) \xi^{2}\right), \\
\tilde{g}_{H W W}^{(5)}= & 0 .
\end{aligned}
$$

From the high-energy viewpoint, the $C P$-odd anomalous couplings $\Delta g_{H V V}^{(1,2,3)}$ of the SM comes from operators $\overline{\mathcal{L}}_{\tilde{B} \boldsymbol{\Sigma}, \tilde{W} \boldsymbol{\Sigma}, \tilde{1}, \tilde{2}, \tilde{3}}$ for the NMCHM.

\section{3. $H V V V$ anomalous couplings}

The anomalous couplings of the Higgs to the gauge bosons, i.e., $H V V V$ can be parametrized as [69]

$$
\begin{aligned}
\mathcal{L}_{\mathrm{eff}, C P}^{\mathrm{HVVV}}= & g_{H W W Z}^{(1)}\left[i\left(\partial_{\mu} W^{-\mu}\right)\left(Z_{\nu} W^{+\nu}\right) h+\text { H.c. }\right] \\
& +g_{H W W A}^{(1)}\left[i\left(\partial_{\mu} W^{-\mu}\right)\left(A_{\nu} W^{+\nu}\right) h+\text { H.c. }\right] \\
& +g_{H W W Z}^{(2)}\left[i\left(Z_{\mu} W^{+\mu}\right)\left(W_{\nu}^{-} \partial^{\nu} h\right)+\text { H.c. }\right] \\
& +g_{H W W A}^{(2)}\left[i\left(A_{\mu} W^{+\mu}\right)\left(W_{\nu}^{-} \partial^{\nu} h\right)+\text { H.c. }\right]
\end{aligned}
$$

where the anomalous couplings $g_{H V V V}$ [69]

$$
\begin{aligned}
& \Delta g_{H W W Z}^{(1)}=\frac{e^{2} g^{2}}{c_{\theta}^{2}}\left(\frac{8}{v} \bar{c}_{6} \xi-\frac{4}{v} \bar{c}_{6} \xi^{2}\right), \\
& \Delta g_{H W W A}^{(1)}=-e g^{2}\left(\frac{8}{v} \bar{c}_{6} \xi-\frac{4}{v} \bar{c}_{6} \xi^{2}\right), \\
& \Delta g_{H W W Z}^{(2)}=-\frac{e^{2} g}{2 c_{\theta}}\left(\frac{16}{v} \bar{c}_{6} \xi-\frac{32}{v} \bar{c}_{6} \xi^{2}\right), \\
& \Delta g_{H W W A}^{(2)}=\frac{e g^{2}}{2}\left(\frac{16}{v} \bar{c}_{6} \xi-\frac{32}{v} \bar{c}_{6} \xi^{2}\right) .
\end{aligned}
$$

All of the $H V V V$ anomalous couplings originate from one singlet operator $\overline{\mathcal{L}}_{6}$ from a high-energy viewpoint, instead of depending upon different EWCL operators, such as $\mathcal{L}_{9,10}$, respectively. 


\section{HHVV anomalous couplings}

The anomalous couplings of the two Higgs to two gauge bosons, i.e., $H H V V$ can be parametrized as [69]

$$
\begin{aligned}
\mathcal{L}_{\mathrm{eff}, C P}^{\mathrm{HHVV}}= & g_{H H W W}^{(1)} W_{\mu}^{+} W^{-\mu} h^{2}+g_{H H W W}^{(2)} W_{\mu}^{+} W^{-\mu} \square\left(h^{2}\right) \\
& +g_{H H W W}^{(3)} W_{\mu}^{+} W_{\nu}^{-} \partial^{\mu} h \partial^{\nu} h \\
& +g_{H H W W}^{(4)}\left(\partial_{\mu} W^{+\mu}\right)\left(\partial_{\nu} W^{-\nu}\right) h^{2} \\
& +g_{H H W W}^{(5)}\left[\left(\partial_{\mu} W^{+\mu}\right)\left(W_{\nu}^{-} \partial^{\nu} h\right) h+\text { H.c. }\right] \\
& +g_{H H Z Z}^{(1)} Z_{\mu} Z^{\mu} h^{2}+g_{H H Z Z}^{(2)} Z_{\mu} Z^{\mu} \square h^{2} \\
& +g_{H H Z Z}^{(3)}\left(Z_{\mu} \partial^{\mu} h\right)^{2}+g_{H H Z Z}^{(4)}\left(\partial_{\mu} Z^{\mu}\right)^{2} h^{2} \\
& +g_{H H Z Z}^{(5)}\left(\partial_{\mu} Z^{\mu}\right)\left(Z_{\nu} \partial^{\nu} h\right) h^{2},
\end{aligned}
$$

where the only nonvanishing couplings at the tree level in the SM are

$$
g_{H H W W}^{(1) S M}=\frac{g^{2}}{4}, \quad g_{H H Z Z}^{(1) S M}=\frac{g^{2}}{8 c_{\theta}^{2}} .
$$

The anomalous couplings of $g_{H H V V}$ turns out to be [69]

$$
\begin{aligned}
\Delta g_{H H W W}^{(1)} & =10 g^{2} m_{h}^{2} \bar{c}_{6} \xi, \\
\Delta g_{H H W W}^{(2)} & =-\frac{12 g^{2}}{v^{2}} \bar{c}_{6} \xi^{2}, \\
\Delta g_{H H W W}^{(3)} & =0, \\
\Delta g_{H H W W}^{(4)} & =-\frac{4 g^{2}}{v^{2}} \bar{c}_{6} \xi+\frac{4 g^{2}}{v^{2}} \bar{c}_{6} \xi^{2}, \\
\Delta g_{H H W W}^{(5)} & =\frac{24 g^{2}}{v^{2}} \bar{c}_{6} \xi^{2} ; \\
\Delta g_{H H Z Z}^{(1)} & =\frac{5 g^{2} m_{h}^{2}}{c_{\theta}^{2}} \bar{c}_{6} \xi, \\
\Delta g_{H H Z Z}^{(2)} & =-\frac{6 g^{2}}{v^{2} c_{\theta}^{2}} \bar{c}_{6} \xi^{2}, \\
\Delta g_{H H Z Z}^{(3)} & =0, \\
\Delta g_{H H Z Z}^{(4)} & =-\frac{2 g^{2}}{v^{2} c_{\theta}^{2}} \bar{c}_{6} \xi+\frac{2 g^{2}}{v^{2} c_{\theta}^{2}} \bar{c}_{6} \xi^{2}, \\
\Delta g_{H H Z Z}^{(5)} & =\frac{24 g^{2}}{v^{2} c_{\theta}^{2}} \bar{c}_{6} \xi^{2} .
\end{aligned}
$$

It is intriguing to observe that all of the $H H V V$ anomalous couplings also originate from one singlet operator $\overline{\mathcal{L}}_{6}$ from a high-energy viewpoint, even though they may obtain contributions from different EWCL operators, such as $\mathcal{L}_{\square H, 7,8,9,10 \text {. }}$

\section{CONCLUSIONS}

In summary, we have studied the effective field theory in the minimal composite Higgs model based upon the $S O(6) / S O(5)$ symmetry breaking pattern up to the $p^{4}$ order in the CCWZ formalism. The vacuum misalignment is parametrized by a rotation angle in the coset space in the effective Lagrangian framework. In order to match with the electroweak chiral Lagrangian, we reparametrize the pseudo-Goldstone boson fields as $(h, s)$, and obtain the connection between the high-energy effective Lagrangian and the low-energy effective electroweak chiral Lagrangian with the Higgs function dependences. By expanding in $v / f$, in the weak coupling limit $(f \rightarrow \infty)$, one would expect to recover the linearly realized SM Higgs theory.

Regarding the connection between the high-energy effective Lagrangian for the NMCHM and the low-energy effective chiral Lagrangian, several main results are in order:

(i) Both $C P$-even and $C P$-odd operators, and both the Omega and Sigma parametrizations are considered, and exact relations between definitions of polar, Cartesian, and mixing coordinates of fields are provided.

(ii) All the exact Higgs functions in the electroweak chiral Lagrangian are presented, which exactly recover the Higgs function in the $S O(5) / S O(4)$ composite Higgs model, in the absence of singlet $s$.

(iii) The Higgs functions in the effective Lagrangian incorporate the Higgs nonlinearity/vacuum misalignment effects in the next-to-minimal composite Higgs model.

(iv) Higgs self couplings, anomalous triple and quartic gauge couplings, anomalous couplings of Higgs to gauge bosons are given as criteria for detecting the physics beyond the SM.

(v) Higgs functions provide relations among different Wilson coefficients in the low-energy chiral Lagrangian. These relations may provide analysis directions for future experimental measurements.

(vi) The singlet can be light and plays a role as a dark matter candidate, in the singlet extended standard model with an additional $Z_{2}$ symmetry in scalar sector.

In the Higgs effective theory, we could obtain the Wilson coefficients derived from the corresponding Higgs functions. At the $p^{2}$ order, the corresponding expansion coefficients are related to the EW oblique parameter $T$ constrained by the EWPT. While at the $p^{4}$ order, they are related to not only the EW oblique parameter $S$ (and also $T$ if heavy fermions are present) parameter but also the anomalous couplings of triple or quartic gauge bosons, the anomalous couplings coefficients of Higgs to gauge bosons, etc. Some of these couplings are constrained by the precision measurements of the Higgs self couplings at the 
LHC. The future colliders will also be able to explore some of these couplings, such as VVH and VVHH couplings. We will leave this study to future work. Finally the phenomenology of the scalar singlet could be quite interesting, such as the collider searches for such scalar, and its cosmological implications, etc.

\section{ACKNOWLEDGMENTS}

We appreciate Hao-Lin Li for early collaboration and valuable discussion of this work. We would like thank Brando Bellazzini, Csaba Csaki, and Jing Shu for valuable discussions. In particularly, Y. H. Q. would like to thank Yu-Ping Kuang for instructing him on theoretical elementary particle physics. He would like to thank XingChang Song and Zhong-Qi Ma for teaching him Lie groups and Lie algebras. Y.H.Q. is supported by the Korean Ministry of Education, Science and Technology, Gyeongsangbuk-do Provincial Government, and Pohang City Government for Independent Junior Research Groups at the Asia Pacific Center for Theoretical Physics (APCTP). J.H.Y. is supported in part by the National Science Foundation of China under Grant No. 11875003 and the Chinese Academy of Sciences (CAS) Hundred-Talent Program. S. H. Z. is supported in part by the National Science Foundation of China under Grants No. 11635001 and No. 11875072.

\section{APPENDIX A: SO(N) GENERATORS AND HIGGS REPRESENTATION}

\section{Generators of the $S O(6)$ group}

In the $\mathrm{SO}(6)$, there are 15 generators $T^{A}=\left\{T^{a}, T^{\hat{a}}\right\}$ denoted with 10 generators of the unbroken $S O(5)$ as $T^{a}=$ $\left\{T_{L / R}^{a}, T^{\alpha}\right\}$ with $a=1,2,3, \alpha=1,2,3,4$ and 5 generators of coset $S O(6) / S O(5)$ as $T^{\hat{a}}=\left\{T^{\hat{\alpha}}, T^{\hat{5}}\right\}$ with $\hat{\alpha}=1,2,3,4$. Among these generators, $\left\{T_{L / R}^{a}\right\}$ belongs to the $S U(2)_{L} \times$ $S U(2)_{R} \simeq S O(4) \subset S O(5)$, and $\left\{T^{\alpha}\right\}$ belong to the coset $S O(5) / S O(4) . T^{\hat{5}}$ belongs to the $S O(2) \subset S O(6)$. These generators satisfy the normalization conditions as

$$
\operatorname{Tr}\left[T^{a} T^{b}\right]=\delta^{a b}, \quad \operatorname{Tr}\left[T^{\hat{a}} T^{\hat{b}}\right]=\delta^{\hat{a} \hat{b}} .
$$

Note, in general, the $S O(6)$ generators satisfy the following commutation relations and trace properties as

$$
\begin{aligned}
{\left[T^{A}, T^{B}\right] } & =i f^{A B C} T^{C}, \quad\left\{T^{A}, T^{B}\right\}=k \delta^{A B}+d^{A B C} T^{C}, \\
\operatorname{Tr}\left[T^{A}\right] & =0, \quad \operatorname{Tr}\left[T^{A} T^{B}\right]=\delta^{A B}, \\
\operatorname{Tr}\left[T^{A} T^{B} T^{C}\right] & =i f^{A B C}+d^{A B C},
\end{aligned}
$$

where $k$ is a normalization constant to be determined, and $f^{A B C}$ and $d^{A B C}$ are totally antisymmetric (symmetric) structure constants, respectively.
The 5 generators of the coset $S O(6) / S O(5)$ can be written in a compact form as $[26,72]$

$$
\left(T^{\hat{a}}\right)_{i j} \equiv \frac{1}{\sqrt{2}}\left(T^{\hat{a} \sigma}\right)_{i j}=\frac{i}{\sqrt{2}}\left(\delta_{i}^{6} \delta_{j}^{\hat{a}}-\delta_{j}^{6} \delta_{i}^{\hat{a}}\right),
$$

where $\hat{a}=1, \ldots, 5, i, j=1, \ldots 6$. It can also be expressed as $\mathbf{5}=\mathbf{4}+\mathbf{1}_{S} \in S O(4) \oplus S O(2)$ as

$$
\begin{aligned}
& \left(T^{\hat{\alpha}}\right)_{i j} \equiv \frac{1}{\sqrt{2}}\left(T^{\hat{\alpha} 6}\right)_{i j}=\frac{i}{\sqrt{2}}\left(\delta_{i}^{6} \delta_{j}^{\hat{\alpha}}-\delta_{j}^{6} \delta_{i}^{\hat{\alpha}}\right), \quad \hat{\alpha}=1,2,3,4, \\
& \left(T^{\hat{5}}\right)_{i j} \equiv \frac{1}{\sqrt{2}}\left(T^{\hat{5} \hat{\sigma}}\right)_{i j}=\frac{i}{\sqrt{2}}\left(\delta_{i}^{6} \delta_{j}^{\hat{5}}-\delta_{j}^{6} \delta_{i}^{\hat{\gamma}}\right),
\end{aligned}
$$

where $T^{\hat{5}} \equiv T_{S}$ is the generator of $S O(2)$, where subscript $S$ denotes singlet. If the unbroken symmetry is $S O(4) \times$ $S U(2)$ with 9 generators or $S O(4) \times S O(2)$ with 7 generators instead of $S O(5)$ with 10 generators, then there are 6,8 instead of 5 GBs that will be present in the end. If one breaks $S O(6)$ down to instead $S O(5)$ but $S O(4) \times S O(2)$, the symmetry breaking pattern $S O(6) / S O(4) \times S O(2)$ leads to $\mathbf{4}+\mathbf{4}$ consisting of the elements of a composite 2-Higgs-doublet model [14]. In this case, $T^{\hat{\alpha}}$ become the unbroken generators.

The 5 generators of the coset $S O(6) / S O(5)$ in a $6 \times 6$ matrix includes $\mathbf{3} \in S U(2)_{\alpha} \subset S O(4)$ as

$$
\begin{gathered}
T^{\hat{1}}=\frac{i}{\sqrt{2}}\left(\begin{array}{cccc}
0_{2} & 0_{2} & & -e_{1} \\
0_{2} & 0_{2} & & \\
& & 0 & \\
e_{1}^{T} & & & 0
\end{array}\right), \\
T^{\hat{2}}=\frac{i}{\sqrt{2}}\left(\begin{array}{llll}
0_{2} & 0_{2} & & -e_{2} \\
0_{2} & 0_{2} & & \\
e_{2}^{T} & & 0 & 0
\end{array}\right), \\
T^{\hat{3}}=\frac{i}{\sqrt{2}}\left(\begin{array}{llll}
0_{2} & 0_{2} & & \\
0_{2} & 0_{2} & & -e_{1} \\
& & 0 & \\
& e_{1}^{T} & & 0
\end{array}\right),
\end{gathered}
$$

where $0_{2} \equiv \operatorname{diag}(0,0)$, and $e_{1,2}$ are eigenvectors of a Pauli matrix as

$$
e_{1}=(1,0)^{T}, \quad e_{2}=(0,1)^{T}
$$

The broken generator along the EW symmetry breaking direction is denoted as $T^{\hat{4}}$ as a $6 \times 6$ matrix as 


$$
\begin{aligned}
& T^{\hat{4}}=\frac{i}{\sqrt{2}}\left(\begin{array}{cccc}
0_{2} & 0_{2} & & \\
0_{2} & 0_{2} & & -e_{2} \\
& & 0 & \\
& e_{2}^{T} & & 0
\end{array}\right), \\
& T^{\hat{5}}=\frac{i}{\sqrt{2}}\left(\begin{array}{cccc}
0_{2} & 0_{2} & \\
0_{2} & 0_{2} & & \\
& & 0 & -1 \\
& & 1 & 0
\end{array}\right),
\end{aligned}
$$

where $T^{\hat{5}}$ is the generator of $S O(2)$ and $0_{2} \equiv \operatorname{diag}(0,0)$.

It can be checked that all generators are normalized as

$\operatorname{Tr}\left[T_{L}^{a} T_{L}^{b}\right]=\delta^{a b}, \quad \operatorname{Tr}\left[T_{R}^{a} T_{R}^{b}\right]=\delta^{a b}, \quad \operatorname{Tr}\left[T^{\hat{a}} T^{\hat{b}}\right]=\delta^{\hat{a} \hat{b}}$,

and they satisfy the commutation relations as below:

$$
\begin{aligned}
{\left[T_{L}^{a}, T_{R}^{b}\right] } & =0, \quad\left[T_{L}^{a}, T_{L}^{b}\right]=i \epsilon^{a b c} T_{L}^{c}, \quad\left[T_{R}^{a}, T_{R}^{b}\right]=i \epsilon^{a b c} T_{R}^{c}, \\
{\left[T^{\hat{a}}, T^{\hat{4}}\right] } & =\frac{i}{2} \delta_{b}^{\hat{a}}\left(T_{L}^{b}-T_{R}^{b}\right), \quad\left[T^{\hat{a}}, T^{\hat{b}}\right]=\frac{i}{2} \epsilon^{\hat{a} \hat{b} c}\left(T_{L}^{c}+T_{R}^{c}\right), \\
{\left[T^{\hat{a}}, T_{L, R}^{b}\right] } & =\frac{i}{2}\left(\epsilon^{\hat{a} b \hat{c}} T^{\hat{c}} \mp \delta^{\hat{a} b} T^{\hat{4}}\right), \quad\left[T^{\hat{4}}, T_{L, R}^{a}\right]= \pm \frac{i}{2} \delta^{a \hat{b}} T^{\hat{b}} .
\end{aligned}
$$

It is obvious that in the $S O(6) / S O(5)$ case, the unbroken generators transform as the reducible representations $(\mathbf{3}, \mathbf{1})+(\mathbf{1}, \mathbf{3}) \in S O(4) \sim S U(2)_{L} \times S U(2)_{R}$, respectively.

Under the unitary transformation $P$ as will be discussed in Eq. (A20), the 5 generator of coset $S O(6) /$ $S O(5)$ in Eqs. (A5) and (A7) can be expressed explicitly as

$$
\begin{aligned}
& t^{\hat{1}}=-\frac{1}{2}\left(\begin{array}{cccc}
0_{2} & 0_{2} & & e_{2} \\
0_{2} & 0_{2} & & e_{1} \\
& & 0 & \\
e_{2}^{T} & e_{1}^{T} & & 0
\end{array}\right) \text {, } \\
& \hat{t^{2}}=\frac{i}{2}\left(\begin{array}{cccc}
0_{2} & 0_{2} & & -e_{2} \\
0_{2} & 0_{2} & & e_{1} \\
& & 0 & \\
e_{2}^{T} & -e_{1}^{T} & & 0
\end{array}\right) \text {, } \\
& \hat{t}=\frac{1}{2}\left(\begin{array}{cccc}
0_{2} & 0_{2} & & -e_{1} \\
0_{2} & 0_{2} & & e_{2} \\
& & 0 & \\
-e_{1}^{T} & e_{2}^{T} & & 0
\end{array}\right) \text {, }
\end{aligned}
$$

$$
\begin{aligned}
t^{\hat{4}} & =\frac{i}{2}\left(\begin{array}{cccc}
0_{2} & 0_{2} & & e_{1} \\
0_{2} & 0_{2} & & e_{2} \\
& & 0 & \\
-e_{1}^{T} & -e_{2}^{T} & & 0
\end{array}\right), \\
t^{\hat{5}} & =\frac{i}{2}\left(\begin{array}{cccc}
0_{2} & 0_{2} & & \\
0_{2} & 0_{2} & & \\
& & 0 & \sqrt{2} \\
& & -\sqrt{2} & 0
\end{array}\right),
\end{aligned}
$$

where $T^{\hat{5}}$ is the generator of $S O(2)$ and $0_{2} \equiv \operatorname{diag}(0,0)$. In the unitary of NMCHM, $h_{1,2,3}=0$, the Higgs PNGBs can be expressed explicitly as

$U=\exp \left(i \frac{\sqrt{2}}{f}\left(h_{4} t^{\hat{4}}+h_{5} t^{\hat{5}}\right)\right)$

$=\left(\begin{array}{cccccc}\frac{c_{\phi}+1}{2} c_{\psi}^{2}+s_{\psi}^{2} & 0 & 0 & \frac{c_{\phi}-1}{2} c_{\psi}^{2} & -\frac{\left(1-c_{\phi}\right) c_{\psi} s_{\psi}}{\sqrt{2}} & -\frac{c_{\psi} s_{\phi}}{\sqrt{2}} \\ 0 & 1 & 0 & 0 & 0 & 0 \\ 0 & 0 & 1 & 0 & 0 & 0 \\ \frac{c_{\phi}-1}{2} c_{\psi}^{2} & 0 & 0 & \frac{c_{\phi}+1}{2} c_{\psi}^{2}+s_{\psi}^{2} & -\frac{\left(1-c_{\phi}\right) c_{\psi} s_{\psi}}{\sqrt{2}} & -\frac{c_{\psi} s_{\phi}}{\sqrt{2}} \\ -\frac{\left(1-c_{\phi}\right) c_{\psi} s_{\psi}}{\sqrt{2}} & 0 & 0 & -\frac{\left(1-c_{\phi}\right) c_{\psi} s_{\psi}}{\sqrt{2}} & c_{\psi}^{2}+c_{\phi} s_{\psi}^{2} & -s_{\phi} s_{\psi} \\ \frac{c_{\psi} s_{\phi}}{\sqrt{2}} & 0 & 0 & \frac{c_{\psi} s_{\phi}}{\sqrt{2}} & s_{\phi} s_{\psi} & c_{\phi}\end{array}\right)$,

where $c_{\phi}=\cos (\phi / f), s_{\phi}=\sin (\phi / f)$, with $\phi=h_{4}^{2}+h_{5}^{2}$ and $\hat{h}_{4}=c_{\psi}, \hat{h}_{5}=s_{\psi}$. In the absence of the singlet $h_{5}=0, \psi=0, \phi=h_{4}=h$, or $h_{4}=0, \phi=h_{5}=s$, then

$$
\begin{aligned}
U(h) & =\left(\begin{array}{cccccc}
\frac{1}{2}\left(c_{h}+1\right) & 0 & 0 & \frac{1}{2}\left(c_{h}-1\right) & 0 & -\frac{s_{h}}{\sqrt{2}} \\
0 & 1 & 0 & 0 & 0 & 0 \\
0 & 0 & 1 & 0 & 0 & 0 \\
\frac{1}{2}\left(c_{h}-1\right) & 0 & 0 & \frac{1}{2}\left(c_{h}+1\right) & 0 & -\frac{s_{h}}{\sqrt{2}} \\
0 & 0 & 0 & 0 & 1 & 0 \\
\frac{s_{h}}{\sqrt{2}} & 0 & 0 & \frac{s_{h}}{\sqrt{2}} & 0 & c_{h}
\end{array}\right), \\
U(s) & =\left(\begin{array}{ccc}
\mathbf{1}_{4} & 0 & 0 \\
0 & c_{s} & -s_{s} \\
0 & s_{s} & c_{s}
\end{array}\right),
\end{aligned}
$$

where $\mathbf{1}_{4}=\operatorname{diag}(1,1,1,1)$. They just recover the GBs matrix for the coset $S O(6) / S O(4)$ or $S O(6) / S O(2)$, respectively. 


\section{Generators of the $S O(5)$ group}

There are 10 unbroken generators of $S O(5) \subset S O(6)$,

$$
\begin{aligned}
\left(T_{L / R}^{a}\right)_{i j} & =-\frac{i}{2}\left(\frac{1}{2} \epsilon^{a b c}\left(\delta_{i}^{b} \delta_{j}^{c}-\delta_{j}^{b} \delta_{i}^{c}\right) \pm\left(\delta_{i}^{a} \delta_{j}^{4}-\delta_{j}^{a} \delta_{i}^{4}\right)\right) \\
\left(T^{\alpha}\right)_{i j} & \equiv \frac{1}{\sqrt{2}}\left(T^{\alpha 5}\right)_{i j}=-\frac{i}{\sqrt{2}}\left(\delta_{j}^{5} \delta_{i}^{\alpha}-\delta_{i}^{5} \delta_{j}^{\alpha}\right),
\end{aligned}
$$

where $a=1,2,3, \alpha=1,2,3,4$ and $i, j=1, \ldots, 6$. These give the coset space $S O(5) / S O(4)$ for MCHM $[13,17,18,20]$.

To be explicit, the 6 generators that span the representation $\mathbf{6}=(\mathbf{3}, 1)+(1, \mathbf{3}) \in S U(2)_{L} \times S U(2)_{R} \simeq S O(4) \subset$ $S O(5) \subset S O(6)$ can be chosen as

$T_{L}^{1}=\frac{i}{2}\left(\begin{array}{ccc}0 & -\sigma_{1} & \\ \sigma_{1} & 0 & \\ & & 0_{2}\end{array}\right), \quad T_{L}^{2}=\frac{i}{2}\left(\begin{array}{ccc}0 & \sigma_{3} & \\ -\sigma_{3} & 0 & \\ & & 0_{2}\end{array}\right)$, $T_{L}^{3}=\frac{i}{2}\left(\begin{array}{ccc}-i \sigma_{2} & 0 & \\ 0 & -i \sigma_{2} & \\ & & 0_{2}\end{array}\right)$;

$T_{R}^{1}=\frac{i}{2}\left(\begin{array}{ccc}0 & i \sigma_{2} & \\ i \sigma_{2} & 0 & \\ & & 0_{2}\end{array}\right), \quad T_{R}^{2}=\frac{i}{2}\left(\begin{array}{ccc}0 & 1_{2} & \\ -1_{2} & 0 & \\ & & 0_{2}\end{array}\right)$, $T_{R}^{3}=\frac{i}{2}\left(\begin{array}{ccc}-i \sigma_{2} & 0 & \\ 0 & i \sigma_{2} & \\ & & 0_{2}\end{array}\right)$,

where $0_{2} \equiv \operatorname{daig}(0,0), 1_{2} \equiv \operatorname{daig}(1,1)$, and it can be checked that they satisfy the commutation relations as

$\left[T_{a}^{L}, T_{b}^{R}\right]=0, \quad T_{a}^{L, R} T_{b}^{L, R}=\frac{1}{4} \delta_{a b}+\frac{i}{2} \varepsilon_{a b c} T_{c}^{L, R}$.

Four residue unbroken generators that span the representation $(\mathbf{2}, \mathbf{2}) \in S U(2)_{L} \times S U(2)_{R} \simeq S O(4)$ are

$$
\begin{aligned}
& T_{\alpha}^{1}=\frac{i}{\sqrt{2}}\left(\begin{array}{cccc}
0_{2} & 0_{2} & -e_{1} & \\
0_{2} & 0_{2} & & \\
e_{1}^{T} & & 0 & \\
& & & 0
\end{array}\right), \\
& T_{\alpha}^{2}=\frac{i}{\sqrt{2}}\left(\begin{array}{llll}
0_{2} & 0_{2} & -e_{2} & \\
0_{2} & 0_{2} & & \\
e_{2}^{T} & & 0 & \\
& & & 0
\end{array}\right),
\end{aligned}
$$

$$
\begin{aligned}
& T_{\alpha}^{3}=\frac{i}{\sqrt{2}}\left(\begin{array}{cccc}
0_{2} & 0_{2} & & \\
0_{2} & 0_{2} & -e_{1} & \\
& e_{1}^{T} & 0 & \\
& & & 0
\end{array}\right), \\
& T_{\alpha}^{4}=\frac{i}{\sqrt{2}}\left(\begin{array}{cccc}
0_{2} & 0_{2} & & \\
0_{2} & 0_{2} & -e_{2} & \\
& e_{2}^{T} & 0 & \\
& & & 0
\end{array}\right),
\end{aligned}
$$

where $0_{2} \equiv \operatorname{diag}(0,0)$.

\section{Generators of the $\mathrm{SO}(4)$ group}

The generator of $S U(2)_{L} \times S U(2)_{R} \simeq S O(4)$ in Eq. (A13) can also be expressed as

$$
\left(T_{L / R}^{a}\right)_{i j}=\frac{1}{2}\left[\left(J^{a}\right)_{i j} \pm\left(K^{a}\right)_{i j}\right],
$$

where the generators $J^{a}$ and $K^{a}$ correspond to the angular momentum and boosts as

$$
\begin{aligned}
\left(J^{a}\right)_{i j} & \equiv \frac{1}{2} \epsilon_{a b c}\left(T^{b c}\right)_{i j}=-i \frac{1}{2} \epsilon_{a b c}\left(\delta_{i}^{b} \delta_{j}^{c}-\delta_{j}^{b} \delta_{i}^{c}\right), \\
\left(K^{a}\right)_{i j} & \equiv\left(T^{a 4}\right)_{i j}=-i\left(\delta_{i}^{a} \delta_{j}^{4}-\delta_{j}^{a} \delta_{i}^{4}\right) .
\end{aligned}
$$

To be explicit,

$$
J^{1}=i\left(\begin{array}{cccc}
0 & 0 & 0 & \\
0 & 0 & -1 & \\
0 & 1 & 0 & \\
& & & 0_{3}
\end{array}\right), \quad J^{2}=i\left(\begin{array}{cccc}
0 & 0 & 1 & \\
0 & 0 & 0 & \\
-1 & 0 & 0 & \\
& & & 0_{3}
\end{array}\right) \text {, }
$$

$$
J^{3}=i\left(\begin{array}{cccc}
0 & -1 & 0 & \\
1 & 0 & 0 & \\
0 & 0 & 0 & \\
& & & 0_{3}
\end{array}\right)
$$

$$
\begin{aligned}
& K^{1}=i\left(\begin{array}{ccccc}
0 & 0 & 0 & -1 & \\
0 & 0 & 0 & 0 & \\
0 & 0 & 0 & 0 & \\
1 & 0 & 0 & 0 & \\
& & & & 0_{2}
\end{array}\right), K^{2}=i\left(\begin{array}{ccccc}
0 & 0 & 0 & 0 & \\
0 & 0 & 0 & -1 & \\
0 & 0 & 0 & 0 & \\
0 & 1 & 0 & 0 & \\
& & & & 0_{2}
\end{array}\right), \\
& K^{3}=i\left(\begin{array}{ccccc}
0 & 0 & 0 & 0 & 0 \\
0 & 0 & 0 & -1 & \\
0 & 0 & 1 & 0 & \\
& & & & 0_{2}
\end{array}\right),
\end{aligned}
$$

where $0_{3} \equiv \operatorname{diag}(0,0,0)$ and $0_{2} \equiv \operatorname{diag}(0,0)$. 
The representation in Eq. (A14) can be transformed to those one is more familiar with through a similar transformation with a unitary matrix $P$,

$$
\begin{aligned}
t_{L, R}^{a} & =P T_{L, R}^{a} P^{-1}, \\
P & =\frac{1}{\sqrt{2}}\left(\begin{array}{cccccc}
0 & 0 & i & 1 & 0 & 0 \\
i & -1 & 0 & 0 & 0 & 0 \\
i & 1 & 0 & 0 & 0 & 0 \\
0 & 0 & -i & 1 & 0 & 0 \\
0 & 0 & 0 & 0 & \sqrt{2} & 0 \\
0 & 0 & 0 & 0 & 0 & -\sqrt{2}
\end{array}\right) .
\end{aligned}
$$

To be explicit, we have

$$
\begin{aligned}
& t_{L}^{1}=\frac{1}{2}\left(\begin{array}{lll}
\sigma_{1} & & \\
& \sigma_{1} & \\
& & 0_{2}
\end{array}\right), \quad t_{L}^{2}=\frac{1}{2}\left(\begin{array}{lll}
\sigma_{2} & & \\
& \sigma_{2} & \\
& & 0_{2}
\end{array}\right), \\
& t_{L}^{3}=\frac{1}{2}\left(\begin{array}{lll}
\sigma_{3} & & \\
& \sigma_{3} & \\
& & 0_{2}
\end{array}\right), \\
& t_{R}^{1}=-\frac{1}{2}\left(\begin{array}{lll}
1_{2} & & \\
& 1_{2} & \\
& & 0_{2}
\end{array}\right), \quad t_{R}^{2}=\frac{i}{2}\left(\begin{array}{lll}
1_{2} & & \\
t_{R}^{3}=\frac{1}{2} & & \\
& 1_{2} & \\
& & 0_{2}
\end{array}\right),
\end{aligned}
$$

where $1_{2} \equiv \operatorname{diag}(1,1)$ and $0_{2} \equiv \operatorname{diag}(0,0)$. Under the above unitary transformation $P$ in Eq. (A20), the fundamental representation of the scalar in $S O(5) \subset S O(6)$ becomes

$$
\phi=\left(\begin{array}{c}
h_{1} \\
h_{2} \\
h_{3} \\
h_{4} \\
s \\
0
\end{array}\right) \stackrel{P}{\rightarrow} \frac{1}{\sqrt{2}}\left(\begin{array}{c}
h_{4}+i h_{3} \\
i\left(h_{1}+i h_{2}\right) \\
h_{2}+i h_{1} \\
h_{4}-i h_{3} \\
\sqrt{2} s \\
0
\end{array}\right)=\left(\begin{array}{c}
h^{0 \star} \\
-h^{-} \\
h^{+} \\
h^{0} \\
s \\
0
\end{array}\right),
$$

which consists of the Higgs bi-doublet as denoted in Eq. (A47) as

$$
\frac{1}{\sqrt{2}} \mathbf{H}=\frac{1}{\sqrt{2}}\left(H^{c}, H\right)=\left(\begin{array}{cc}
h^{0 \star} & h^{+} \\
h^{-} & h^{0}
\end{array}\right),
$$

where $H$ and $H^{c}$ are the SM Higgs doublet and its complex conjugate with notation defined in Eqs. (A33) and (A46), respectively.

Under the above unitary transformation $P$ in Eq. (A20), the 4 generators in the coset $S O(5) / S O(4)$ in Eq. (A24) can be expressed more explicitly as

$$
\begin{aligned}
t_{\alpha}^{1} & =\frac{1}{2}\left(\begin{array}{llll}
0_{2} & 0_{2} & e_{2} & \\
0_{2} & 0_{2} & e_{1} & \\
e_{2}^{T} & e_{1}^{T} & 0 & \\
& & & 0
\end{array}\right), \\
t_{\alpha}^{2} & =\frac{i}{2}\left(\begin{array}{cccc}
0_{2} & 0_{2} & e_{2} & \\
0_{2} & 0_{2} & -e_{1} & \\
-e_{2}^{T} & e_{1}^{T} & 0 & \\
& & & 0
\end{array}\right), \\
t_{\alpha}^{3} & =\frac{1}{2}\left(\begin{array}{cccc}
0_{2} & 0_{2} & e_{1} & \\
0_{2} & 0_{2} & -e_{2} & \\
e_{1}^{T} & -e_{2}^{T} & 0 & \\
& & & 0
\end{array}\right), \\
t_{\alpha}^{4} & =\frac{i}{2}\left(\begin{array}{llll}
0_{2} & 0_{2} & -e_{1} & \\
0_{2} & 0_{2} & -e_{2} & \\
e_{1}^{T} & e_{2}^{T} & 0 & \\
& & & 0
\end{array}\right),
\end{aligned}
$$

where $0_{2} \equiv \operatorname{diag}(0,0)$. In the unitary of MCHM, $h_{1,2,3}=0$, $h_{4}=h$, the Higgs PNGBs can be expressed explicitly as

$$
\begin{aligned}
U & =\exp \left(i \frac{\sqrt{2}}{f} h t_{\alpha}^{4}\right) \\
& =\left(\begin{array}{cccccc}
\frac{1}{2}\left(c_{h}+1\right) & 0 & 0 & \frac{1}{2}\left(c_{h}-1\right) & \frac{s_{h}}{\sqrt{2}} & 0 \\
0 & 1 & 0 & 0 & 0 & 0 \\
0 & 0 & 1 & 0 & 0 & 0 \\
\frac{1}{2}\left(c_{h}-1\right) & 0 & 0 & \frac{1}{2}\left(c_{h}+1\right) & \frac{s_{h}}{\sqrt{2}} & 0 \\
-\frac{s_{h}}{\sqrt{2}} & 0 & 0 & -\frac{s_{h}}{\sqrt{2}} & c_{h} & 0 \\
0 & 0 & 0 & 0 & 0 & 1
\end{array}\right),
\end{aligned}
$$

where $c_{h}=\cos (h / f)$ and $s_{h}=\sin (h / f)$.

Under the unitary transformation $P$ in Eq. (A20), the 3 generators of angular momentum and boost in Eq. (A19) can be reexpressed as 
$\mathbf{J}^{1}=\frac{1}{2}\left(\begin{array}{ccccc}0 & 1 & -1 & 0 & \\ 1 & 0 & 0 & -1 & \\ -1 & 0 & 0 & 1 & \\ 0 & -1 & 1 & 0 & \\ & & & & 0_{2}\end{array}\right)$

$\mathrm{J}^{2}=\frac{i}{2}\left(\begin{array}{ccccc}0 & -1 & -1 & 0 & \\ 1 & 0 & 0 & -1 & \\ 1 & 0 & 0 & -1 & \\ 0 & 1 & 1 & 0 & \\ & & & & 0_{2}\end{array}\right), \quad \mathbf{J}^{3}=\left(\begin{array}{ccccc}0 & 0 & 0 & 0 & \\ 0 & -1 & 0 & 0 & \\ 0 & 0 & 1 & 0 & \\ 0 & 0 & 0 & 0 & \\ & & & & 0_{2}\end{array}\right)$

$\mathrm{K}^{1}=\frac{1}{2}\left(\begin{array}{lllll}0 & 1 & 1 & 0 & \\ 1 & 0 & 0 & 1 & \\ 1 & 0 & 0 & 1 \\ 0 & 1 & 1 & 0 & \\ & & & & 0_{2}\end{array}\right), \quad \mathrm{K}^{2}=\frac{i}{2}\left(\begin{array}{ccccc}0 & -1 & 1 & 0 & \\ 1 & 0 & 0 & 1 & \\ -1 & 0 & 0 & -1 & \\ 0 & -1 & 1 & 0 & \\ & & & & 0_{2}\end{array}\right)$,

$\mathrm{K}^{3}=\left(\begin{array}{ccccc}1 & 0 & 0 & 0 & \\ 0 & 0 & 0 & 0 & \\ 0 & 0 & 0 & 0 & \\ 0 & 0 & 0 & -1 & \\ & & & & 0\end{array}\right)$,

where $0_{2} \equiv \operatorname{diag}(0,0)$.

\section{Representations of Higgs}

a. Custodial symmetry $S O(3) \subset S O(4)$

As we will show in the following, the unbroken $\mathcal{H}=S O(3) \simeq S U(2)_{V}$ symmetry after EW symmetry breaking originates from an enlarged $\mathcal{G}=S O(4) \simeq$ $S U(2)_{L} \times S U(2)_{R}$ global symmetry.

It is interesting to observe that the Higgs doublet is an $S O(4)$ invariant as

$$
H^{\dagger} H=h_{1}^{2}+h_{2}^{2}+h_{3}^{2}+h_{4}^{2}=h^{T} h,
$$

with $h=\left(h_{1}, h_{2}, h_{3}, h_{4}\right)^{T}$ is $\mathbf{4} \in S O(4)$. Thus the SM Higgs action can also be reexpressed as an $S O(4) \subset$ $S O(5)$ invariant form as

$\mathcal{L}_{h}=\frac{1}{2}\left(D_{\mu} h^{T}\right)\left(D_{\mu} h\right)+\frac{1}{2} \mu^{2}\left(h^{T} h\right)-\frac{1}{4} \lambda\left(h^{T} h\right)^{2} ;$

with $h=\left(h_{1}, h_{2}, h_{3}, h_{4}\right)^{T}$ transforming linearly as the fourdimensional vector representation $\mathbf{4}$ of global symmetry group $S O(4)$, it is obvious that the Higgs potential is also invariant under $S O(4)$ symmetry. The Higgs potential can be reexpressed as

$$
V_{h}=-\frac{\lambda}{4}\left(h^{T} h-v^{2}\right)^{2}
$$

which has a minimum as the three sphere $S^{3}$ with radius $v$

$$
\left\langle h^{T} h\right\rangle=v^{2} .
$$

It is convenient to choose the vacuum expectation value of $h$ in vector representation 4 of $S O(4)$ as

$$
\langle h\rangle=\left(\begin{array}{l}
0 \\
0 \\
0 \\
v
\end{array}\right) .
$$

Considering the shift field $v \rightarrow v+h$ and nonshifted fields as $h_{a}$ with $a=1,2,3$, then

$$
h=\left(\begin{array}{c}
h_{1} \\
h_{2} \\
h_{3} \\
v+h
\end{array}\right),
$$

where $v$ is the vacuum expectation value of the Higgs singlet $h_{4}$ and $h \equiv \tilde{h}_{4}$ is the quantum fluctuation around the $v$. Thus, the $S O(4)$ symmetry acts linearly on the Cartesian coordinates in terms of field $h_{a}$.

The vacuum $\langle h\rangle$ simultaneously breaks the global $S O(4)$ symmetry down to the unbroken $O(3)$ global symmetry, under which, the GBs $\varphi_{a}$ with $a=1,2,3$ transforms linearly as a triplet $\mathbf{3}$ under $O(3)$, while the Higgs $h$ transforms as a singlet $\mathbf{1} \in O(3)$. The four-real component of the Higgs vector representation can be reorganized as the usual SM Higgs complex doublet with two complex scalar fields $h^{+}$and $h_{0}$ as

$$
\begin{gathered}
\Phi=\frac{1}{\sqrt{2}}\left(\begin{array}{c}
h_{2}+i h_{1} \\
h_{4}-i h_{3}
\end{array}\right) \equiv\left(\begin{array}{l}
h^{+} \\
h_{0}
\end{array}\right)=\frac{1}{\sqrt{2}} H \\
\stackrel{\varphi=0}{=} \frac{1}{\sqrt{2}}\left(\begin{array}{c}
0 \\
h+v
\end{array}\right),
\end{gathered}
$$

where $H$ is related to $\Phi=H / \sqrt{2}$, due to the kinetic term, and in the last equality, we have adopted to the unitary gauge.

After EWSB, the $S O(4)$ breaks down to a $S O(3) \simeq$ $S U(2)_{V}$ symmetry, in terms of custodial symmetry, which is invariant for the three goldstone bosons, or as equivalent physical degree of freedom, the three gauge bosons $W_{\mu}^{a}$ in 3 , the fundamental representation of $S O(3)$ or adjoint representation of $S U(2)_{V}$. The unbroken global symmetry $S O(3)$ leads to the well-known gauge boson mass relation that $m_{W}=m_{Z} \cos \theta_{W}$ as the prediction of the SM, which can also be expressed through the ratio 


$$
\rho \equiv \frac{m_{W}^{2}}{m_{Z}^{2} c_{\theta}^{2}}
$$

when $g^{\prime} \neq 0$, where $c_{\theta}=\cos \theta_{W}$ is the Weinberg mixing angle. The gauge boson mass relation implies that custodial is an approximate symmetry of the SM, which is exact at least at tree level. Thus, the custodial symmetry ensures $\rho=1$ at tree level, and also ensures small corrections to $\rho$ at the quantum level in Eq. (1.1). It also guarantees that $m_{W}^{2} / m_{Z}^{2}=1$ in the absence of $U(1)_{Y}$, i.e., $g^{\prime} \rightarrow 0$.

To be brief, the unbroken $S O(3)$ symmetry of the SM vacuum can be originated from an $S O(4)$ invariant fixed point.

\section{b. From Cartesian to polar}

It is worthy of observing a fact that after the $h_{4}$ obtains vev, the SM Higgs doublet with Cartesian coordinate field $h_{a}$ in Eq. (A33) can be reexpressed as below $[40,46,60]$

$$
\begin{aligned}
& \Phi=\frac{1}{\sqrt{2}}\left(\begin{array}{c}
h_{2}+i h_{1} \\
h+v-i h_{3}
\end{array}\right) \\
& =\left(\mathbf{1}_{2}+\frac{h \mathbf{1}_{2}+i \sigma^{a} h_{a}}{v}\right)\left(\begin{array}{c}
0 \\
\frac{v}{\sqrt{2}}
\end{array}\right) \\
& \stackrel{v \gg 1}{\approx} \frac{v}{\sqrt{2}} \exp \left(\frac{h \mathbf{1}_{2}+i \sigma^{a} h_{a}}{v}\right)\left(\begin{array}{l}
0 \\
1
\end{array}\right) \\
& \stackrel{h \ll v}{\approx} \exp \left(\frac{i \sigma^{a} h_{a}}{v}\right) \frac{1}{\sqrt{2}}\left(\begin{array}{c}
0 \\
v+h
\end{array}\right) \text {, }
\end{aligned}
$$

where $e_{2}=(0,1)^{T}$ is one of two eigenvectors of the Pauli matrix $\sigma^{3}$ for a spin-down state as denoted in Eq. (A6) and in the last equality, we have made the identification that $\varphi_{a} \approx h_{\hat{a}}$ with $\hat{a}=1,2,3$, which are exact in the limit $v \rightarrow \infty$, i.e., the unitary gauge. Thus, one can map the Higgs doublet in Eq. (A33) to that in the polar decomposition, or angular coordinate $\varphi_{a}$ as

$$
\Phi \equiv \frac{1}{\sqrt{2}} h_{4} \mathbf{U} e_{2},
$$

where $h_{4}=h+v$ is the magnitude of $\Psi$ where $h$ denotes the radial coordinate, while $\mathbf{U} \in S^{3}$ is a four-dimensional unit vector, denoting the SM Goldstone bosons matrix in $S U(2)$ as defined in Eq. (C2)

$$
\begin{aligned}
\mathbf{U} & \equiv \exp \left(i \frac{\sigma^{a} \varphi_{a}}{v}\right)=c_{\varphi}+i \sigma^{a} \hat{\varphi}_{a} s_{\varphi} \\
& =\left(\begin{array}{cc}
c_{\varphi}+\frac{i s_{\varphi}}{|\varphi|} \varphi_{3} & \frac{s_{\varphi}}{|\varphi|}\left(i \varphi^{1}+\varphi^{2}\right) \\
\frac{i s_{\varphi}}{|\varphi|}\left(\varphi^{1}+i \varphi^{2}\right) & c_{\varphi}-\frac{i s_{\varphi}}{|\varphi|} \varphi_{3}
\end{array}\right) \\
& \stackrel{v \gg 1}{=}\left(\begin{array}{cc}
1+\frac{i \varphi_{3}}{v} & \frac{i \varphi_{1}+\varphi_{2}}{v} \\
\frac{i \varphi_{1}-\varphi_{2}}{v} & 1-\frac{i \varphi_{3}}{v}
\end{array}\right) \stackrel{\varphi=0}{=} \mathbf{1}_{2},
\end{aligned}
$$

where $\hat{\varphi}_{a} \equiv \varphi_{a} /|\varphi|$ are the three dimensionless angular coordinates and we have made the notation $s_{\varphi}$ and $c_{\varphi}$ defined in Eq. (2.54). In the last equality, it is shown that the unitary matrix just reduces to be unity in the unitary gauge, as expected.

The metric of the coset space is $S O(4) / S O(3) \simeq S^{3}$ as

$$
\begin{aligned}
g_{\hat{a} \hat{b}} & \equiv \operatorname{Tr}\left(\partial_{\hat{a}} \mathbf{U}^{\dagger} \partial_{\hat{b}} \mathbf{U}\right) \\
& =\frac{2}{v^{2}}\left[\frac{\phi^{a} \phi^{b}}{|\phi|^{2}}+\left(\delta^{a b}-\frac{\phi^{a} \phi^{b}}{|\phi|^{2}}\right) \frac{v^{2}}{|\phi|^{2}} \sin ^{2}\left(\frac{|\phi|}{v}\right)\right],
\end{aligned}
$$

which gives the determinant as

$$
\operatorname{det}[g(\phi)]=\frac{4}{v^{2}|\phi|^{4}} \sin ^{4}\left(\frac{|\phi|}{v}\right) .
$$

From which, one can define the vielbein from Eq. (5.78) as

$$
\begin{aligned}
E_{a}^{A}= & -i \operatorname{Tr}\left[\Omega^{\dagger} \partial_{\hat{a}} \Omega T^{A}\right] \\
= & \frac{2 \phi_{a} \phi^{A}}{v|\phi|^{2}}+\left(\delta_{a}^{A}-\frac{\phi_{a} \phi^{A}}{|\phi|^{2}}\right) \frac{1}{|\phi|} \sin \left(\frac{2|\phi|}{v}\right) \\
& +2 \epsilon_{a}^{A b} \frac{\phi_{b}}{|\phi|^{2}} \sin ^{2}\left(\frac{|\phi|}{v}\right) .
\end{aligned}
$$

According to Eq. (5.82), the torsion contribution is

$$
T^{A B C}=\frac{4}{v|\phi|^{2}} \sin ^{2}\left(\frac{|\phi|}{v}\right)=4 \epsilon^{A B C} \sqrt{g} .
$$

With the $h$ in doublet $H$ in Eq. (A33), according to Eqs. (A36) and (A37), one can read the one-to-one correspondence as

$$
h_{a}=\frac{h_{4}}{v} \varphi_{a}, \quad a=1,2,3,
$$

where $h_{4}=h+v$ with $h=\tilde{h}_{4}$ as the quantum fluctuation of the Higgs field. In this case, there is a relation between the GBs in Cartesian and polar coordinates as

$$
|h|^{2}=h_{a} h_{a}=\frac{h_{4}^{2}\left(v^{2}+|\varphi|^{2}\right)}{v^{2}} \stackrel{\varphi \rightarrow 0}{=}(h+v)^{2},
$$

which originates from the constraint of the polar coordinate. Therefore,

$$
h=h_{4}-v+h_{4} \frac{\varphi^{2}}{2 v^{2}}-h_{4} \frac{\varphi^{4}}{8 v^{4}}+\cdots .
$$

Thus, the Higgs filed $h$ is $S O(4)$ invariant, while only three of the components of $\Phi\left(h_{a}, h_{4}\right)$ are independent due to the constraint above. 
In the polar coordinate, the SM Lagrangian can be reexpressed as

$$
\begin{aligned}
\mathcal{L}_{h}= & \frac{1}{2}(v+h)^{2}\left(\partial_{\mu} \mathbf{U}^{\dagger} \partial^{\mu} \mathbf{U}\right) \\
& +\frac{1}{2}\left(\partial_{\mu} h\right)^{2}-\frac{\lambda}{4}\left((h+v)^{2}-v^{2}\right)^{2},
\end{aligned}
$$

where the Higgs scalar potential depends only upon the radial coordinate $h$, while the three GB fields $\varphi$ only appears in the kinetic terms, either gauged or not. In this case, it is obvious that the interactions of $h$ with $\varphi^{a}$ becomes nonlinear, compared to that between $h_{a}$ and $h_{4}$ in the Cartesian coordinate. Nevertheless, by the LehmannSymanzik-Zimmermann reduction formula, $h$ and $h_{4}$ give the same $S$-matrix, so that the change of coordinates does not affect $S$-matrix elements [60].

\section{c. Higgs bi-doublet in $\mathrm{SO}(4)$}

Since $S O(4)$ is isomorphic to $S U(2)_{L} \times S U(2)_{R}$, one can relate the $S O(4)$ vector to $\mathbf{H} \equiv\left(H^{c}, H\right)$ a complex $S U(2)_{R} \times S U(2)_{L}$ bi-doublet $(\overline{2}, 2) \in S U(2)_{L} \times S U(2)_{R}$, with the complex conjugate of $H$ in Eq. (A33) as

$$
H^{c} \equiv i \sigma^{2} H^{\star}=\left(\begin{array}{c}
h_{4}+i h_{3} \\
-\left(h_{2}-i h_{1}\right)
\end{array}\right)=\left(\begin{array}{c}
h^{0 *} \\
h^{-}
\end{array}\right) \text {, }
$$

where $h^{-}=\left(h^{+}\right)^{*}$. More explicitly, the polar decomposition of $\mathbf{H}$ into $h$ and the $S U(2)$ matrix $U$ as

$\mathbf{H}=\left(\begin{array}{cc}h_{4}+i h_{3} & h_{2}+i h_{1} \\ -h_{2}+i h_{1} & h_{4}-i h_{3}\end{array}\right)=h_{\hat{\alpha}} \bar{\sigma}_{\hat{\alpha}} \equiv|h| \mathbf{U}$

with $\bar{\sigma}_{\hat{\alpha}}=\left(i \vec{\sigma}, \mathbf{1}_{2}\right)$ with $\hat{\alpha}=(a, 4), a=1,2,3$.

From Eq. (A47), it can be found that

$$
\frac{h_{\hat{\alpha}}}{|h|} \equiv \hat{h}_{\hat{\alpha}}=\frac{1}{2} \operatorname{Tr}\left[\mathbf{U} \sigma_{\hat{\alpha}}\right],
$$

where $\sigma_{\hat{\alpha}} \equiv\left(-i \vec{\sigma}, \mathbf{1}_{2}\right)$ is the complex conjugate of $\bar{\sigma}_{\hat{\alpha}}$.

To be explicit, $\hat{h}_{\hat{\alpha}}$ can also be expressed as, respectively,

$$
\hat{h}_{a}=-\frac{i}{2} \operatorname{Tr}\left[\mathbf{U} \sigma_{a}\right], \hat{h}_{4}=c_{\varphi} .
$$

Combing Eqs. (A37), the $h_{a}$ in the vector representation can be expressed as

$$
\hat{h}_{\hat{\alpha}}=\left(\hat{\varphi}_{a} s_{\varphi}, c_{\varphi}\right) \stackrel{v \rightarrow \infty}{=}\left(\frac{\varphi_{a}}{v}, 1\right),
$$

where $\hat{\varphi}_{a} \equiv \varphi_{a} /|\varphi|$ and $c_{\varphi}, s_{\varphi}$ as defined in Eq. (2.54). Therefore, the fundamental scalar $h^{T}$ can be reexpressed as

$$
\phi=v\left(\begin{array}{c}
s_{\varphi} \vec{\varphi} \\
c_{\varphi}
\end{array}\right)
$$

where $\vec{\varphi} \equiv\left(\hat{\varphi}_{1}, \hat{\varphi}_{2}, \hat{\varphi}_{3}\right)$ is a three-dimensional unit vector, which transforms linearly as the three-dimensional representation under the unbroken $O(3)$ symmetry. The angle $\varphi$ together with the $\vec{\varphi}$ have 4 degrees of freedom.

\section{d. Nonlinear transformation of $\mathrm{SO}(4)$}

Since there are three independent components in the polar coordinate with the constraint in Eq. (A43), one can make a redefinition of the GBs as below,

$$
\pi_{a} \equiv v s_{\varphi} \hat{\varphi}_{a},
$$

so that the fourth nonindependent component can be expressed as a nonlinear function of these unconstrained fields $\pi_{a}$. In this case, the linear parametrization in Eq. (A51) can be reexpressed in a square-root parametrization as

$$
\phi=\left(\frac{\vec{\pi}}{\sqrt{v^{2}-\vec{\pi} \cdot \vec{\pi}}}\right),
$$

where $\vec{\pi}$ has three components. Thus, the unitary GB matrix $\mathbf{U}$ can also be rewritten in the nonlinear form as

$$
\mathbf{U}=\sqrt{1-\frac{\pi^{2}}{v^{2}}}+i \sigma^{a} \frac{\pi_{a}}{v},
$$

which implies that the fourth component $c_{\varphi}$ is a nonlinear function of the independent three components $\pi^{a}$ with $a=1,2,3$. The constraint of polar coordinate in Eq. (A43) turns the $S O(4)$ linear transformation upon $\Phi\left(h_{a}, h_{4}\right)$ into a nonlinear $S O(4)$ transformation when written only in terms of unconstrained fields $\pi^{1,2,3} \in S O(3)$.

Thus, the $S O(4)$ symmetry acts nonlinearly on the angular coordinates in terms of field $\pi_{a}$ reads as

$$
\mathbf{U} \approx 1+i \frac{\pi}{v}-\frac{\pi^{2}}{2 v^{2}}+\mathcal{O}\left(\frac{\pi^{4}}{v^{4}}\right)
$$

where $\pi \equiv \sigma^{a} \pi_{a}$.

In this definition, the kinetic terms of the nonlinear sigma model can be rewritten as

$$
\begin{aligned}
\mathcal{L}_{\text {kin }} & =\frac{v^{2}}{2} \operatorname{Tr}\left[\left(\partial_{\mu} \mathbf{U}^{\dagger}\right)\left(\partial^{\mu} \mathbf{U}\right)\right] \\
& =\frac{1}{2}\left(\partial_{\mu} \pi^{a}\right)^{2}+\frac{v^{2}}{2} s_{\varphi}\left(\partial_{\mu} \varphi\right)^{2},
\end{aligned}
$$

where the second term denotes the nonlinear kinetic terms of $\pi^{a}$ fields, since 


$$
\mathcal{L}_{\text {kin }}^{(\varphi)}=\frac{v^{2}}{2} s_{\varphi}\left(\partial_{\mu} \varphi\right)^{2}=\frac{1}{2} \frac{\left(\pi^{a} \partial_{\mu} \pi^{a}\right)^{2}}{v^{2}-\pi^{2}} .
$$

Together, the kinetic term of $\pi^{a}$ becomes in curved space $S^{3} \simeq S O(4) / S O(3)$ as

$$
\mathcal{L}_{\text {kin }}=\frac{1}{2} g_{a b} \partial_{\mu} \pi^{a} \partial^{\mu} \pi^{b},
$$

with a nonflat metric in PNGB field space

$$
g_{a b}=\delta_{a b}+\frac{\pi_{a} \pi_{b}}{v^{2}-\pi^{2}},
$$

and the Ricci scalar curvature of the coset space is

$$
R=\frac{6}{v^{2}}>0
$$

where the number 6 takes account of the degrees of freedom of the number of unconstrained $\pi^{a}$ fields. Therefore, the PNGB, or Higgs nonlinearity originates from the scalar $S^{3}$ manifold curvature. At the scale $v \rightarrow \infty$, the physics at scale $f$ gives nonlinear interactions due to the metric

$$
g_{a b}=\delta_{a b}+\frac{\pi_{a} \pi_{b}}{v^{2}}+\frac{\pi^{2}}{v^{4}} \pi_{a} \pi_{b}+\mathcal{O}\left(\frac{\pi^{6} \delta_{a b}}{v^{6}}\right) .
$$

The kinetic term of the nonlinear sigma model can be expressed in a series of expansions as

$$
\begin{aligned}
\mathcal{L}_{\text {kin }}^{(\varphi)}= & \frac{1}{2} \partial_{\mu} \vec{\pi} \cdot \partial^{\mu} \vec{\pi}+\frac{1}{2 v^{2}}\left(\vec{\pi} \cdot \partial_{\mu} \vec{\pi}\right)^{2} \\
& +\frac{1}{2 v^{4}} \vec{\pi} \cdot \vec{\pi}\left(\vec{\pi} \cdot \partial_{\mu} \vec{\pi}\right)^{2}+\ldots,
\end{aligned}
$$

where ... denotes higher order derivatives coupled interaction terms no less than $1 / v^{6}$ order. The Lagrangian has only three scalar degrees of freedom.

\section{e. Higgs function in coset $S O(4) / S O(3)$}

Similarly, if the SM GBs are introduced in the coset $S O(4) / S O(3)$ with the nonlinear realization, as parametrized in Eq. (A54), the same story above would happen, namely the GBs also originate from the nonflat metric induced by the EW scale $v$ as shown in Eq. (A60).

The kinetic energy term of the Higgs can be proposed from that in the coset as Eq. (A58) to the $S O(4)$ manifold, by introducing additional Higgs in fundamental $S O(4) H$ as the quantum fluctuations along the radial field direction [39,58-60],

$$
\mathcal{L} \equiv \frac{1}{2} g_{\alpha \beta}(h) \partial_{\mu} \phi^{\alpha} \partial^{\mu} \phi^{\beta},
$$

where $\phi^{\alpha}=\left(\pi^{a}, h\right)$ and $g_{\alpha \beta}(h)$ is a general metric on $S O(4)$,

$$
g_{\alpha \beta}=\operatorname{diag}\left(F(h)^{2} g_{a b}, g_{h h}\right),
$$

where $F(h)$ is an arbitrary function of radial coordinate filed $h$ canonically normalized so that allows one to set $F(0)=1$ and $g_{h h}=1 . g_{a b}$ is the $S O(4)$ invariant metric on the scalar coset manifold $S O(4) / S O(3)=S^{3}$ in Eq. (A59). In this case, the Riemann tensors $R_{\alpha \beta \gamma \delta}$ are

$$
\begin{aligned}
& R_{b c d}^{a} \propto\left(1-v^{2} F^{\prime 2}\right)\left(g_{c}^{a} g_{b d}-g_{d}^{a} g_{b c}\right), \\
& R_{h b h}^{a} \propto F^{\prime \prime} g_{b}^{a},
\end{aligned}
$$

where $F^{\prime} \equiv \partial F / \partial h, F^{\prime \prime} \equiv \partial^{2} F / \partial h^{2}$ etc. are derivatives of $F$ with respect to $h$. The Ricci tensors $R_{\alpha \beta}$ are

$$
\begin{aligned}
& R_{a b} \propto \frac{1}{F^{2}}\left(\frac{2}{v^{2}}-2 F^{\prime 2}-F F^{\prime \prime}\right) g_{a b}, \\
& R_{h h} \propto \frac{F^{\prime \prime}}{F},
\end{aligned}
$$

and the intrinsic Ricci scalar curvature $R$ turns out to be

$$
R=\frac{6}{v^{2}}\left(\frac{1}{F^{2}}-\frac{v^{2}}{F^{2}}\left(F^{\prime 2}+F F^{\prime \prime}\right)\right)
$$

The exterior curvature with a normal vector along the radial coordinate direction gives

$$
K_{b}^{a}\left(n^{c}\right)=\frac{\pi^{c}}{F} \delta_{b}^{a},
$$

where $\delta^{a}{ }_{b}$ are diagonal components of the induced metric. Thus, the extrinsic curvature is flat as before. While the minimization of the extrinsic curvature along radial direction will entail that $F \neq 0$. The Einstein equations $G_{a b}=0$ and $G_{h h}=0$ impose the constraints, respectively, as

$$
2 F F^{\prime \prime}+F^{\prime 2}=\frac{1}{v^{2}}, \quad F^{\prime}=\frac{1}{v},
$$

where the second one originates from the vanishing of the Riemann tensor curvature $R_{b c d}^{a}$ in Eq. (A65) above. These equations together give the solution to $F(h)$ for the Higgs kinetic energy term

$$
F_{\mathrm{SM}}(v)=C+\frac{h}{v},
$$

where integral constant $C=1$ is determined by imposing $F(0)=1$. This just reproduces the Higgs function in front of the kinetic terms of the SM would-be Goldstone bosons in Eq. (5.11), where the degrees of freedom of GBs are 
transferred to the gauge boson masses after EWSB due to Higgs mechanism.

When expanding the nonflat metric of SM GBs in Eq. (A63) in power series of $1 / v$, we just obtain the

$$
\begin{aligned}
\mathcal{L}= & \frac{1}{2}\left(\partial_{\mu} h\right)\left(\partial^{\mu} h\right)+\frac{1}{2}\left(1+\frac{h}{v}\right)^{2} \partial_{\mu} \vec{\pi} \cdot \partial^{\mu} \vec{\pi} \\
& +\frac{1}{v^{2}}\left(1+\frac{h}{v}\right)^{2}\left(\vec{\pi} \cdot \partial_{\mu} \vec{\pi}\right)^{2}+\ldots,
\end{aligned}
$$

where ... denotes the higher order multi-interaction terms. It is worthy of noticing that the GBs are derivatively coupled.

\section{APPENDIX B: CCWZ AND CHIRAL LAGRANGIAN}

\section{Nambu-Goldstone boson matrix}

\section{a. $\Omega$ parametrization}

Assuming the Lagrangian is invariant under global $\mathcal{G}$, with an unbroken symmetry $\mathcal{H} \subset \mathcal{G}$, which is a linearly realized subgroup of $\mathcal{G}$. The Nambu-Goldstone bosons due to the global spontaneous symmetry breaking pattern $\mathcal{G} \rightarrow \mathcal{H}$ can be described by the $\Omega$ field defined as

$$
\Omega \equiv e^{i \frac{\varphi}{2 f} \Xi} \equiv e^{i \Pi},
$$

where in the last equality, we have absorbed the decay constant $f$ into $\Pi$ so that it is a dimensionless field. The $\Omega$ is under the action of an element of global symmetry $\mathfrak{g} \in \mathcal{G}$ and an element of local symmetry $\mathfrak{h} \in \mathcal{H}$ as [27,28]

$$
\mathfrak{g} \Omega(\Xi)=\Omega(g(\Xi)) \mathfrak{h}(\Xi, g),
$$

which transforms in a linear representation for $H$. While it is a nonlinear one for the other elements under the global symmetry $\mathcal{G}$ as

$$
\Omega \rightarrow \mathfrak{g} \Omega \mathfrak{h}^{-1}(\Xi, \mathfrak{g}),
$$

which defines the nonlinear transformation of the NGBs fields as $\Xi$ denoted as

$$
\Xi=\Xi^{\hat{a}} T_{\hat{a}},
$$

where $T_{\hat{a}}$ are the broken generators in the coset $\mathcal{G} / \mathcal{H}$, with $\hat{a}=1, \ldots, \operatorname{dim}(\mathcal{G} / \mathcal{H})$. To be brief, the Nambu-Goldstone boson fields are associated with the coset or quotient space. The quotient space $\mathcal{G} / \mathcal{H}$ is said to be symmetric if there exists an automorphism of the grading, $\mathcal{R}$, under which the broken generators change sign.

\section{b. Symmetric coset and automorphism}

For symmetric coset, there exists an automorphism or "grading" symmetry $\mathcal{R}$ that acts upon the generators of $\mathcal{G}$, which changes the sign of the broken generators as

$$
\mathcal{R}:\left\{\begin{array}{l}
T_{a} \rightarrow+T_{a} \\
T_{\hat{a}} \rightarrow-T_{\hat{a}}
\end{array}\right.
$$

where $T_{a}$ with $a=1,2, \ldots, \operatorname{dim}(\mathcal{H})$ are the generators of $\mathcal{H}$ and $T_{\hat{a}}$ where $\hat{a}=1,2, \ldots, \operatorname{dim}(\mathcal{G} / \mathcal{H})$ are the generators of the coset $\mathcal{G} / \mathcal{H}$. The $\left(T_{a}, T_{\hat{a}}\right)$ form an orthonormal basis of $\mathcal{G}$, and they satisfy

$$
\left[T_{a}, T_{b}\right] \propto T_{c}, \quad\left[T_{a}, T_{\hat{b}}\right] \propto T_{\hat{c}}, \quad\left[T_{\hat{a}}, T_{\hat{b}}\right] \propto T_{c},
$$

where the first condition follows from that $\mathcal{H}$ is closed, the second condition is due to the fact that the structure constants are completely antisymmetric for compact groups, while the last condition is one for a symmetric coset. These generators together consist of those for global symmetry $\mathcal{G}$ with the commutations relations and trace conditions in Eq. (A2).

To be explicit, the commutation relations for the unbroken and broken generators of a group $\mathcal{G}$ with $T^{A}=$ $\left\{T^{a}, T^{\hat{a}}\right\}$ are

$$
\begin{aligned}
& {\left[T_{a}, T_{b}\right]=i f_{a b}^{c} T_{c},} \\
& {\left[T_{a}, T_{\hat{b}}\right]=i f_{a \hat{b}}{ }^{\hat{b}} T_{\hat{c}}+i f_{a \hat{a}}{ }^{c} T_{c},} \\
& {\left[T_{\hat{a}}, T_{\hat{b}}\right]=i f_{\hat{a} \hat{b}}{ }^{c} T_{c}+i f_{\hat{a} \hat{b}}{ }^{c} T_{\hat{c}},}
\end{aligned}
$$

where the structure constants $f_{a b}{ }^{\hat{c}}=0$ since the unbroken generators $\left\{T^{a}\right\}$ forms a subgroup $\mathcal{H} \subset \mathcal{G}$. For noncompact group $\mathcal{G}$, e.g., $S O(5,1)$, the structure constants $f_{A B}{ }^{C}=$ $-f_{B A}{ }^{C}$ are antisymmetric in their first two indices. For compact groups, e.g., $S O(6)$, the structure constants in all three indices are totally antisymmetric. Thus, the structure constant $f_{a \hat{b}}{ }^{c}=0$. The second commutation relation reduces to

$$
\left[T_{a}, T_{\hat{b}}\right]=i f_{a \hat{b}}^{\hat{c}} T_{\hat{c}} .
$$

The antisymmetric constant can be expressed in terms of matrix elements of unbroken generators in the adjoint representation as $f_{a b}{ }^{c}=-i\left(t_{a}\right)^{c}{ }_{b}$. For the symmetric coset case, the broken generator $T^{\hat{a}}$ changes its sign. As a result, not only the structure constant $f_{a \hat{b}}{ }^{c}=0$ vanishes, but also $f_{\hat{a} \hat{b}} \hat{c}=0$. The third commutation relation reduces to

$$
\left[T_{\hat{a}}, T_{\hat{b}}\right]=i f_{\hat{a} \hat{b}}^{c} T_{c} .
$$

Under the discrete automorphism symmetry $\mathcal{R}$ in Eq. (B5), the GB fields transforms as 


$$
\mathcal{R}: \Omega \rightarrow \Omega^{-1} \text {. }
$$

In the symmetric coset, the nonlinear field transformation of $\Omega$ can be read as below by acting on Eq. (B3) with the grading $\mathcal{R}$ and taking the Hermitian conjugate

$$
\Omega \rightarrow \mathfrak{h} \Omega \mathfrak{g}_{\mathcal{R}}^{-1}
$$

\section{c. $\Sigma$ parametrization}

From Eqs. (B11) and (B3), one can define a new nonlinear field $\boldsymbol{\Sigma}$ as square of $\Omega$

$$
\boldsymbol{\Sigma} \equiv \Omega^{2}=e^{i \frac{\varphi}{f} \Xi}=e^{2 i \Pi},
$$

which transforms linearly under the global symmetry $\mathfrak{g} \in \mathcal{G}$ as

$$
\Sigma \rightarrow \mathfrak{g} \boldsymbol{\Sigma}_{\mathcal{R}}^{-1},
$$

where $\mathfrak{g}_{\mathcal{R}}$ is a global element of $\mathcal{G}$, obtained by acting on $g$ with $\mathcal{R}$, which is independent of the GBs field $\Pi$. Hence $\boldsymbol{\Sigma}$ transforms linearly under $\mathcal{G}$. This shows explicitly that the transformation on $\Pi$ is a realization of $\mathcal{G}$ and that it is linear when restricted to $\mathcal{H}$. Under the grading symmetry, the GB fields transform as

$$
\mathcal{R}: \boldsymbol{\Sigma} \rightarrow \boldsymbol{\Sigma}^{-1} .
$$

The transformation also implies that the covariant derivative of the nonlinear $\boldsymbol{\Sigma}$ is defined as

$$
\mathbf{D}_{\mu} \boldsymbol{\Sigma}=\partial_{\mu} \boldsymbol{\Sigma}+i g_{A}\left(\overline{\mathrm{A}}_{\mu} \boldsymbol{\Sigma}-\boldsymbol{\Sigma} \overline{\mathrm{A}}_{\mu}^{(\mathcal{R})}\right)
$$

where $\overline{\mathrm{A}}_{\mu}$ is the $\mathcal{G}$ gauge field and its automorphism field is $\overline{\mathrm{A}}_{\mu}^{\mathcal{R}} \equiv \mathcal{R}\left(\overline{\mathrm{A}}_{\mu}\right)$. Under the gauge transformation, it is straightforward to have

$$
\begin{aligned}
\overline{\mathrm{A}}_{\mu} & \rightarrow \mathfrak{g} \overline{\mathrm{A}}_{\mu} \mathfrak{g}^{-1}-\frac{i}{g_{A}} \mathfrak{g}\left(\partial_{\mu} \mathfrak{g}^{-1}\right), \\
\overline{\mathrm{A}}_{\mu}^{\mathcal{R}} & \rightarrow \mathfrak{g}_{R} \overline{\mathrm{A}}_{\mu}^{\mathcal{R}} \mathfrak{g}_{\mathcal{R}}^{-1}-\frac{i}{g_{A}} \mathfrak{g}_{\mathcal{R}}\left(\partial_{\mu} \mathfrak{g}_{\mathcal{R}}^{-1}\right),
\end{aligned}
$$

with $g_{A}$ denoting the associated gauge coupling constant.

For external gauge field $g_{A} \overline{\mathrm{A}}_{\mu}=A_{\mu}$, where the gauge coupling $g_{A}$ are absorbed into the gauge fields. Consequently, the covariant derivative of $\boldsymbol{\Sigma}$ in Eq. (B15) transformed under local $\mathcal{G}$ transformation are defined as

$$
D_{\mu} \boldsymbol{\Sigma}=\partial_{\mu} \boldsymbol{\Sigma}+i A_{\mu} \boldsymbol{\Sigma}-i \boldsymbol{\Sigma} A_{\mu}^{(\mathcal{R})},
$$

where $A_{\mu}=A_{\mu}^{a} T^{a}+A_{\mu}^{\hat{a}} T^{\hat{a}}$ is the external gauge field and $A_{\mu}^{(\mathcal{R})}$ is obtained by acting on $A_{\mu}$ with the "grading" $\mathcal{R}$ as

$$
A_{\mu}^{(\mathcal{R})}=\mathcal{R}\left(A_{\mu}\right)=A_{\mu}^{a} T^{a}-A_{\mu}^{\hat{a}} T^{\hat{a}},
$$

where $T^{\hat{a}}$ and $T^{a}$ are the broken and unbroken generators, respectively and normalized as $\operatorname{Tr}\left(T^{A} T^{B}\right)=\delta^{A B}$. In gauging the standard model group, i.e., $A_{\mu}^{\hat{a}}=0$. The external gauge field $A_{\mu}$ transforms under the local $\mathcal{H}$ symmetry as

$$
\begin{aligned}
A_{\mu} & \rightarrow \mathfrak{g} A_{\mu} \mathfrak{g}^{-1}-i \mathfrak{g} \partial_{\mu} \mathfrak{g}^{-1}, \\
A_{\mu}^{(\mathcal{R})} & \rightarrow \mathfrak{g}_{(\mathcal{R})} A_{\mu} \mathfrak{g}_{(\mathcal{R})}^{-1}-i \mathfrak{g}_{(\mathcal{R})} \partial_{\mu} \mathfrak{g}_{(\mathcal{R})}^{-1} .
\end{aligned}
$$

Therefore, the covariant term of $\boldsymbol{\Sigma}$ above can be expressed as

$$
D_{\mu} \boldsymbol{\Sigma}=\partial_{\mu} \boldsymbol{\Sigma}+i\left[A_{\mu}^{a} T^{a}, \boldsymbol{\Sigma}\right]+i\left\{A_{\mu}^{\hat{a}} T^{\hat{a}}, \boldsymbol{\Sigma}\right\} .
$$

\section{d. Explicit expression of covariant derivative}

The most general high-energy effective Lagrangian for describing the electroweak interactions of the gauge group $\mathcal{G}$ and of the GBs of a nonlinear realization of the symmetric $\mathcal{G} / \mathcal{H}$ basis, up to four-derivative bosonic interactions, can be obtained by gauging only the EW symmetry of the SM gauge group, while keeping the group $\mathcal{G}=S O(6)$ global.

The gauge covariant derivative as defined in Eq. (B20) becomes

$$
D_{\mu} \boldsymbol{\Sigma}=\partial_{\mu} \boldsymbol{\Sigma}+i\left[W_{\mu}^{a} Q_{L}^{a}+B_{\mu}^{3} Q_{Y}, \boldsymbol{\Sigma}\right],
$$

where the external gauge field as $g_{A} \overline{\mathrm{A}}_{\mu}=g \overline{\mathrm{W}}_{\mu}+g^{\prime} \overline{\mathrm{B}}_{\mu}$ by using the embedding representations in the SM as Eqs. (2.1) and (2.3), we find the explicit expression of the gauge invariant fields strength as

$g_{A} \overline{\mathrm{A}}_{\mu}=\frac{1}{2}\left(\begin{array}{ccc}\sigma_{2} A_{\mu} & -i \sigma_{1} g W_{\mu}^{1}+i \sigma_{3} g W_{\mu}^{2} & \\ i \sigma_{1} g W_{\mu}^{1}-i \sigma_{3} g W_{\mu}^{2} & \sigma_{2} Z_{\mu} & \\ & & 0_{2}\end{array}\right)$,

where $A_{\mu} \equiv g W_{\mu}^{3}+g^{\prime} B_{\mu}, Z_{\mu} \equiv g W_{\mu}^{3}-g^{\prime} B_{\mu}$, and $0_{2}=\operatorname{daig}(0,0)$. Then, we can obtain the field stress tensor of the $\operatorname{SM} g_{A} \overline{\mathrm{F}}_{\mu \nu} \equiv$ $\left(g \overline{\mathrm{W}}_{\mu \nu}, g^{\prime} \overline{\mathrm{B}}_{\mu \nu}\right)$ in a similar expression.

The $S O(6) / S O(5)$ explicit expression of the building block with the SM gauge group is

$$
\overline{\mathrm{V}}_{\mu}(\Omega)=\left(\begin{array}{cc}
0_{3} & \overline{\mathrm{V}}_{\mu}^{(W, B)} \\
-\overline{\mathrm{V}}_{\mu}^{(W, B) T} & \overline{\mathrm{V}}_{\mu}^{(\phi, \psi)}
\end{array}\right),
$$

where $0_{3}=\operatorname{diag}(0,0,0)$, and $\overline{\mathrm{V}}_{\mu}^{(W, B)}$ and $\overline{\mathrm{V}}_{\mu}^{(\phi, \psi)}$ are both $3 \times 3$ matrixes those can be expressed, respectively, as 


$$
\begin{aligned}
& \overline{\mathrm{V}}_{\mu}^{(W, B)}=\frac{1}{2}\left(\begin{array}{ccc}
g c_{\psi}^{2}\left(1-c_{\phi}\right) W_{\mu}^{1} & g\left(1-c_{\phi}\right) s_{\psi} c_{\psi} W_{\mu}^{1} & g c_{\psi} s_{\phi} W_{\mu}^{1} \\
g c_{\psi}^{2}\left(1-c_{\phi}\right) W_{\mu}^{2} & g\left(1-c_{\phi}\right) s_{\psi} c_{\psi} W_{\mu}^{2} & g c_{\psi} s_{\phi} W_{\mu}^{2} \\
c_{\psi}^{2}\left(1-c_{\phi}\right) Z_{\mu} & \left(1-c_{\phi}\right) s_{\psi} c_{\psi} Z_{\mu} & c_{\psi} s_{\phi} Z_{\mu}
\end{array}\right), \\
& \overline{\mathrm{V}}_{\mu}^{(\phi, \psi)}=\frac{1}{f}\left(\begin{array}{ccc}
0 & 0 & c_{\psi} \\
0 & 0 & s_{\psi} \\
-c_{\psi} & -s_{\psi} & 0
\end{array}\right) \phi_{\mu}+\left(\begin{array}{ccc}
0 & c_{\phi}-1 & -s_{\phi} s_{\psi} \\
1-c_{\phi} & 0 & c_{\psi} s_{\phi} \\
s_{\phi} c_{\psi} & -c_{\psi} s_{\phi} & 0
\end{array}\right) \psi_{\mu},
\end{aligned}
$$

where $\phi_{\mu}=\partial_{\mu} \phi$ and $\psi_{\mu}=\partial_{\mu} \psi$. The scalar chiral field $\overline{\mathbf{T}}$ in Eq. (B38) becomes

$$
\overline{\mathbf{T}}=-\frac{i}{2}\left(\begin{array}{cccccc}
0 & 1 & 0 & 0 & 0 & 0 \\
-1 & 0 & 0 & 0 & 0 & 0 \\
0 & 0 & 0 & -1+c_{\psi}^{2}\left(1-c_{\phi}\right) & \left(1-c_{\phi}\right) s_{\psi} c_{\psi} & c_{\psi} s_{\phi} \\
0 & 0 & 1-c_{\psi}^{2}\left(1-c_{\phi}\right) & 0 & 0 & 0 \\
0 & 0 & -\left(1-c_{\phi}\right) s_{\psi} c_{\psi} & 0 & 0 & 0 \\
0 & 0 & -c_{\psi} s_{\phi} & 0 & 0 & 0
\end{array}\right)
$$

\section{2. $\Omega$ decomposition}

In the CCWZ's general approach, one can redefine the GB fields as $d_{\mu}$ and $e_{\mu}$ through the Maurer-Cartan (MC) one-form $\omega$, which is decomposed along the broken and unbroken directions, respectively,

$$
\omega_{\mu} \equiv-i \Omega^{\dagger} \partial_{\mu} \Omega=d_{\mu}^{\hat{a}} T^{\hat{a}}+e_{\mu}^{a} T^{a} \equiv d_{\mu}+e_{\mu},
$$

where $\Omega$ is defined in Eq. (B3) as defined in Eq. (2.5) and the kinetic term can be generalized to be a covariant derivative term. $T^{\hat{a}} \in \mathcal{G} / \mathcal{H}$ while $T^{a} \in \mathcal{H}$ are respectively the broken and unbroken generators normalized as $\operatorname{Tr}\left(T^{A} T^{B}\right)=\delta^{A B}$. In the group space manifold, $\left(d_{\mu}^{\hat{a}}, e_{\mu}^{a}\right)$ can be viewed as the vielbeins of $\mathcal{G}$, where $e_{\mu}^{a}$ are the vielbeins of $\mathcal{H}$, and $d_{\mu}^{\hat{a}}$ are those of the coset $\mathcal{G} / \mathcal{H}$ vacuum manifold.

Since $\Omega(\Pi, \mathfrak{g})$ is transformed under global symmetry $\mathcal{G}$ as Eq. (B3),

$$
\begin{aligned}
& -i \Omega^{-1} \partial_{\mu} \Omega \rightarrow-i \mathfrak{h} \Omega^{-1} \mathfrak{g}^{-1} \partial_{\mu}\left(\mathfrak{g} \Omega \mathfrak{h}^{-1}\right) \\
& =-i \mathfrak{h} \Omega^{-1} \partial_{\mu}\left(\Omega \mathfrak{h}^{-1}\right) \\
& =-i \mathfrak{h} \Omega^{-1}\left(\partial_{\mu} \Omega\right) \mathfrak{h}^{-1}-i \mathfrak{h}\left(\partial_{\mu} \mathfrak{h}^{-1}\right) \\
& =\mathfrak{h} \omega_{\mu} \mathfrak{h}^{-1}-i \mathfrak{h}\left(\partial_{\mu} \mathfrak{h}^{-1}\right),
\end{aligned}
$$

where $\mathfrak{h} \omega_{\mu} \mathfrak{h}^{\dagger} \in \mathcal{G}$, while the shift term $i \mathfrak{h} \partial_{\mu} \mathfrak{h}^{\dagger} \in \mathcal{H}$.

From the above transformation, together with Eq. (B26), one deduces that under any element $\mathfrak{g} \in \mathcal{G}, d_{\mu}$ and $e_{\mu}$ transform as

$$
\begin{aligned}
d_{\mu} & \rightarrow \mathfrak{h}(\Pi, \mathfrak{g}) d_{\mu} \mathfrak{h}^{-1}(\Pi, \mathfrak{g}), \\
e_{\mu} & \rightarrow \mathfrak{h}(\Pi, \mathfrak{g}) e_{\mu} \mathfrak{h}^{-1}(\Pi, \mathfrak{g})-i h(\Pi, \mathfrak{g}) \partial_{\mu} \mathfrak{h}^{-1}(\Pi, \mathfrak{g}) .
\end{aligned}
$$

Thus, $d_{\mu}$ transforms linearly under local symmetry $\mathcal{H}$ as the NGBs, while $e_{\mu}$ transforms nonlinearly as a gauge field with gauge symmetry $\mathcal{H}$.

It is obvious that the covariant field $e_{\mu}$ transforms like a gauge field under $\mathcal{H}$, i.e., transforms as a connection, and it will be more obvious by defining a field strength

$$
e_{\mu \nu} \equiv \partial_{\mu} e_{\nu}-\partial_{\nu} e_{\mu}+i\left[e_{\mu}, e_{\nu}\right]
$$

where $e_{\mu}$ can be viewed as a nonlinear relation of the gauge field with gauge symmetry $\mathcal{H} . e_{\mu \nu}$ transforms linearly under the local symmetry $\mathcal{H}$ again, like that of $d_{\mu}$ as

$$
e_{\mu \nu} \rightarrow \mathfrak{h}(\Pi, \mathfrak{g}) e_{\mu \nu} \mathfrak{h}^{-1}(\Pi, \mathfrak{g})
$$

Therefore, $d_{\mu}(\Pi)$ and $e_{\mu \nu}(\Pi)$ are covariant variables, and together with those by acting upon the covariant derivative $\nabla_{\mu}$ consist of the building blocks of the low-energy effective Lagrangian.

Except the covariant derivative $\nabla_{\nu}$ for local symmetry $\mathcal{H}$, one might be interested in gauging an external local symmetry $\mathcal{H}^{\prime} \subset \mathcal{G}$, e.g., a subgroup $S U(2)_{L} \times U(1)_{Y}$, which plays the role of the SM electroweak group. In this case, one can replace the kinetic derivative with the a covariant derivative of the external gauge field $A_{\mu}$ as

$$
D_{\mu}=\partial_{\mu}+i A_{\mu}
$$

where the gauge couplings $g_{A}$ are absorbed into the gauge fields. 


\section{Building blocks in CCWZ}

\section{a. Building blocks for $\Sigma$}

The global vector chiral field (if the interactions are not gauged) is defined as

$$
\overline{\mathrm{V}}_{\mu}=\left(\partial_{\mu} \boldsymbol{\Sigma}\right) \boldsymbol{\Sigma}^{-1}
$$

and it transforms in the adjoint representation of $\mathcal{G}$ as

$$
\overline{\mathrm{V}}_{\mu} \rightarrow \mathfrak{g} \overline{\mathrm{V}}_{\mu} \mathfrak{g}^{-1} \text {. }
$$

The effective Lagrangian describing the GB interactions in the context of the nonlinearly realized $\mathcal{G}$ breaking mechanism, with symmetric coset $\mathcal{G} / \mathcal{H}$ can be constructed from $\overline{\mathrm{V}}_{\mu}$. Then the gauged version of the chiral vector field of the nonlinear sigma model can be defined as through the formally gauging of the full group as

$$
\overline{\mathrm{V}}_{\mu}=\left(\mathbf{D}_{\mu} \boldsymbol{\Sigma}\right) \boldsymbol{\Sigma}^{-1}
$$

The Cartan form for $\mathcal{G}$ of the above

$$
\left(\boldsymbol{\Sigma}^{-1} d \mathbf{\Sigma}\right)^{\hat{a}}
$$

after projecting onto the subspace of broken generators, gives a vielbein corresponding to the metric on $\mathcal{G} / \mathcal{H}$. As will be seen, the leading-order terms with at most two derivatives are built out of two vielbein. The Wess-ZuminoWitten term is a five-form, which can be built out of five vielbein [23].

The building blocks for the effective chiral gauge theory includes a $\mathcal{G}$ invariant vector chiral field $\overline{\mathrm{V}}_{\mu}$ and the gauge field strength $\overline{\mathrm{F}}_{\mu \nu}=\left(\overline{\mathrm{W}}_{\mu \nu}, \overline{\mathrm{B}}_{\mu \nu}\right)$ which transform in the adjoint of $\mathcal{G}$.

The building blocks are

$$
\overline{\mathrm{V}}_{\mu}, \quad \overline{\mathrm{F}}_{\mu \nu}, \quad \boldsymbol{\Sigma} \overline{\mathrm{F}}_{\mu \nu}^{(\mathcal{R})} \boldsymbol{\Sigma}^{-1} .
$$

with the automorphism symmetry in the symmetric coset, one can introduce the graded vector chiral fields

$$
\overline{\mathrm{V}}_{\mu}^{(\mathcal{R})} \equiv \mathcal{R}\left(\overline{\mathrm{V}}_{\mu}\right)=\left(\mathbf{D}_{\mu} \boldsymbol{\Sigma}\right)^{-1} \boldsymbol{\Sigma}=-\boldsymbol{\Sigma}^{-1} \overline{\mathrm{V}}_{\mu} \boldsymbol{\Sigma} .
$$

One additional custodial symmetry breaking source besides the SM ones, in analogy to the scalar chiral field $\mathbf{T}$ in the high energy can be embedded in $\mathcal{G}$ of the hypercharge generators $Q_{Y}$ as

$$
\overline{\mathbf{T}} \equiv \boldsymbol{\Sigma} Q_{Y} \boldsymbol{\Sigma}^{-1} .
$$

To be brief, the building block of the high-energy effective Lagrangian for a UV complete model, i.e., a symmetric CHM are

$$
\overline{\mathrm{V}}_{\mu}, \quad \overline{\mathrm{F}}_{\mu \nu}, \quad \boldsymbol{\Sigma} \overline{\mathrm{F}}_{\mu \nu}^{(\mathcal{R})} \boldsymbol{\Sigma}^{-1}, \quad \overline{\mathbf{T}} .
$$

Based upon these, we can construct the gauge covariant operators in the following.

\section{b. Building blocks for $\boldsymbol{\Omega}$}

In the $\Omega$ parametrization, the building blocks $\overline{\mathrm{V}}_{\mu}$ and $\overline{\mathrm{F}}_{\mu \nu}$ under the adjoint of $\mathcal{G}$, should be transformed in the adjoint of the preserved subgroup $\mathcal{H}$ as

$$
\begin{aligned}
v_{\mu} & \equiv \Omega^{-1} \overline{\mathrm{V}}_{\mu} \Omega=\Omega^{-1} \mathbf{D}_{\mu} \Omega-\Omega \mathbf{D}_{\mu} \Omega^{-1}, \\
v_{\mu}^{(\mathcal{R})} & \equiv \Omega \overline{\mathrm{V}}_{\mu}^{(\mathcal{R})} \Omega^{-1}=\Omega \mathbf{D}_{\mu} \Omega^{-1}-\Omega^{-1} \mathbf{D}_{\mu} \Omega=-v_{\mu}, \\
f_{\mu \nu} & \equiv \Omega^{-1} \overline{\mathrm{F}}_{\mu \nu} \Omega, \quad f_{\mu \nu}^{(\mathcal{R})} \equiv \Omega \overline{\mathrm{F}}_{\mu \nu}^{(\mathcal{R})} \Omega^{-1}, \\
\chi & \equiv \Omega^{-1} \overline{\mathbf{T}} \Omega=\Omega Q_{Y} \Omega^{-1},
\end{aligned}
$$

where $\mathcal{R}$ denotes the automorphism (aut) symmetry and for the custodial breaking chiral scalar $\chi$, we have used the definition of $\overline{\mathbf{T}}$ in Eq. (B38) as well as Eq. (2.24). Since $v_{\mu}^{(R)}=-v_{\mu}$ is not independent of $v_{\mu}$, there are three building blocks in the $\Omega$ parametrization as $\left\{v_{\mu}, f_{\mu \nu}, f_{\mu \nu}^{\mathcal{R}}\right\}$. By defining the aut-even and aut-odd components of $v_{\mu}$ as

$$
v_{\mu}^{ \pm} \equiv \frac{1}{2}\left(v_{\mu} \pm v_{\mu}^{(\mathcal{R})}\right)
$$

from which, we can deduce that $v_{\mu}$ always belongs to the coset sector, i.e., it runs only over the broken generators but does not sum over the unbroken ones at all, as expected

$$
v_{\mu}^{+}=0 \in \mathcal{H}, \quad v_{\mu}^{-}=v_{\mu}=v_{\mu}^{\hat{a}} T^{\hat{a}} \in \mathcal{G} / \mathcal{H} .
$$

Therefore, in the $\boldsymbol{\Sigma}$ parametrization, there are four building block in the $\Omega$ parametrization as $\left\{v_{\mu}, f_{\mu \nu}, f_{\mu \nu}^{(\mathcal{R})}, \chi\right\}$. It is useful to make the notations as below

$$
\begin{aligned}
\Omega^{-1} \mathbf{D}_{\mu} \Omega \equiv \frac{v_{\mu}}{2}+i e_{\mu} & =\frac{1}{2} v_{\mu}^{\hat{a}} T^{\hat{a}}+i e_{\mu}^{a} T^{a}, \\
& =i \omega_{\mu}=i\left(d_{\mu}+e_{\mu}\right),
\end{aligned}
$$

where the factor $1 / 2$ is due to the fact that $v_{\mu}=$ $2 \Omega^{-1} \partial_{\mu} \Omega+\cdots$ where $\ldots$ represents those due to the covariant derivative. Thus, the MC one-form can be expressed as

$$
\omega_{\mu}=e_{\mu}-\frac{i}{2} v_{\mu}, \quad \omega_{\mu}^{(\mathcal{R})}=e_{\mu}+\frac{i}{2} v_{\mu},
$$

which gives 


$$
\begin{aligned}
e_{\mu} & \equiv \frac{1}{2}\left(\omega_{\mu}+\omega_{\mu}^{(\mathcal{R})}\right)=e_{\mu}^{(\mathcal{R})}, \\
v_{\mu} & \equiv i\left(\omega_{\mu}-\omega_{\mu}^{(\mathcal{R})}\right)=-v_{\mu}^{(\mathcal{R})} .
\end{aligned}
$$

Therefore, one has

$$
d_{\mu}=-i \frac{1}{2} v_{\mu}=\frac{1}{2}\left(\omega_{\mu}-\omega_{\mu}^{(\mathcal{R})}\right)
$$

Similar to Eq. (B42), one has

$$
\begin{aligned}
& d_{\mu}^{+}=0 \in \mathcal{H}, \quad d_{\mu}^{-}=d_{\mu}=d_{\mu}^{\hat{a}} T^{\hat{a}} \in \mathcal{G} / \mathcal{H}, \\
& e_{\mu}^{+}=e_{\mu}=e_{\mu}^{a} T^{a} \in \mathcal{H}, \quad e_{\mu}^{-}=0 \in \mathcal{G} / \mathcal{H} .
\end{aligned}
$$

Therefore, $e_{\mu}^{(R)}=e_{\mu}$ is not independent of $e_{\mu}$. In the last equality in Eq. (B43), we have also used the notations in Eq. (B97) with

$$
v_{\mu}=2 i d_{\mu}, \quad e_{\mu}=\bar{e}_{\mu}+\overline{\mathrm{A}}_{\mu},
$$

and $\bar{e}_{\mu}$ transforms under the unbroken symmetry $\mathcal{H}$ as

$$
v_{\mu} \rightarrow \mathfrak{h} v_{\mu} \mathfrak{h}^{-1}, \quad \bar{e}_{\mu} \rightarrow \mathfrak{h}\left(\bar{e}_{\mu}-i \partial_{\mu}\right) \mathfrak{h}^{-1}
$$

Thus, $\bar{e}_{\mu}$ transforms as a connection and it is possible to define the extended covariant derivative of $v_{\mu}$ as

$$
\nabla_{\mu} v_{\nu}=\mathbf{D}_{\mu} v_{\nu}+i\left[\bar{e}_{\mu}, v_{\nu}\right]=\partial_{\mu} v_{\nu}+i\left[e_{\mu}, v_{\nu}\right]
$$

where

$$
\mathbf{D}_{\mu} v_{\nu}=\mathbf{D}_{\mu} v_{\nu}+i\left[\overline{\mathrm{A}}_{\mu}, v_{\nu}\right]
$$

Equation (B50) is consistent with Eq. (B63) by considering that $v_{\nu}=2 i d_{\nu}$, thus,

$$
\nabla_{\mu} d_{\nu}=\partial_{\mu} d_{\nu}+i\left[e_{\mu}, d_{\nu}\right]=\mathbf{D}_{\mu} d_{\nu}+i\left[\bar{e}_{\mu}, d_{\nu}\right]
$$

For the gauge field strength $\overline{\mathrm{F}}_{\mu \nu}=\left(\overline{\mathrm{W}}_{\mu \nu}, \overline{\mathrm{B}}_{\mu \nu}\right)$ under the SM gauge group, as a subgroup of invariant gauge symmetry $\mathcal{H}$, according to Eq. (B40), it is convenient to make the new notations more explicitly for the gauge field strength $f_{\mu \nu}=\left(w_{\mu \nu}, b_{\mu \nu}\right)$ and $f_{\mu \nu}^{(\mathcal{R})}=\left(w_{\mu \nu}^{(\mathcal{R})}, b_{\mu \nu}^{(\mathcal{R})}\right)$ as

$$
\begin{aligned}
w_{\mu \nu} \equiv \Omega^{-1} \overline{\mathbf{W}}_{\mu \nu} \Omega, & b_{\mu \nu} \equiv \Omega^{-1} \overline{\mathrm{B}}_{\mu \nu} \Omega, \\
w_{\mu \nu}^{(\mathcal{R})} \equiv \Omega^{-1} \overline{\mathbf{W}}_{\mu \nu}^{(\mathcal{R})} \Omega, & b_{\mu \nu}^{(\mathcal{R})} \equiv \Omega^{-1} \overline{\mathbf{B}}_{\mu \nu}^{(\mathcal{R})} \Omega,
\end{aligned}
$$

and more over, they can be expressed in terms of aut-even and aut-odd gauge fields as

$$
\begin{array}{ll}
w_{\mu \nu}=w_{\mu \nu}^{+}+w_{\mu \nu}^{-}, & w_{\mu \nu}^{(\mathcal{R})}=w_{\mu \nu}^{+}-w_{\mu \nu}^{-}, \\
b_{\mu \nu}=b_{\mu \nu}^{+}+b_{\mu \nu}^{-}, & b_{\mu \nu}^{(\mathcal{R})}=b_{\mu \nu}^{+}-b_{\mu \nu}^{-} .
\end{array}
$$

Therefore, for the SM gauge group as a subgroup of unbroken symmetry group $\mathcal{H}$, we have $f_{\mu \nu}^{(\mathcal{R})}=$ $\left(w_{\mu \nu}^{(\mathcal{R})}, b_{\mu \nu}^{(\mathcal{R})}\right)$.

With the field strength of the gauge field $\overline{\mathrm{F}}_{\mu \nu}$, in the symmetric coset case, under the grading/automorphism (aut) $\mathcal{R}$ as $f_{\mu \nu}^{(\mathcal{R})} \equiv \mathcal{R}\left(f_{\mu \nu}\right)$, one can also redefine the gauge field as a new covariant structure variables $f_{\mu \nu}^{ \pm}$as

$$
f_{\mu \nu} \equiv f_{\mu \nu}^{-}+f_{\mu \nu}^{+}, \quad f_{\mu \nu}^{(\mathcal{R})} \equiv-f_{\mu \nu}^{-}+f_{\mu \nu}^{+} .
$$

where $f^{ \pm}$are the aut-even and aut-odd components of $f_{\mu \nu}$, and can be viewed as the gauge fields under the $\mathcal{H}$ and $\mathcal{G} / \mathcal{H}$, respectively as

$$
f_{\mu \nu}^{ \pm}=\frac{1}{2}\left(f_{\mu \nu} \pm f_{\mu \nu}^{(\mathcal{R})}\right)
$$

which can be expressed in terms of CCWZ covariant building blocks $d_{\mu}, e_{\mu \nu}$ as explicitly shown in Eq. (B66). The automorphism of these components of $f_{\mu \nu}$ satisfies

$$
\mathcal{R}\left[f_{\mu \nu}^{ \pm}\right]= \pm f_{\mu \nu}^{ \pm}
$$

which also implies that the gauge field $f_{\mu \nu}^{ \pm}$carries even and odd $\mathcal{R}$ parity.

For the symmetry breaking pattern $\mathcal{G} / S O(4)=S U(2)_{L} \times$ $S U(2)_{R}$, one can decompose the gauge fields as [19]

$$
\begin{aligned}
\mathbf{1 0} & =(\mathbf{3}, \mathbf{1})+(\mathbf{1}, \mathbf{3})+\mathbf{4}: f_{\mu \nu}^{+}=f_{\mu \nu}^{L}+f_{\mu \nu}^{R}+f_{\mu \nu}^{(\alpha)}, \\
\mathbf{5} & =\mathbf{4}+\mathbf{1}: f_{\mu \nu}^{-}=f_{\mu \nu}^{(\hat{a})}+f_{\mu \nu}^{(\hat{\alpha})},
\end{aligned}
$$

where $f_{\mu \nu}^{-}=f_{\mu \nu}^{-\hat{a}} T_{\hat{a}} \in \mathcal{G} / \mathcal{H}, f_{\mu \nu}^{+}=f_{\mu \nu}^{+a} T_{a} \in \mathcal{H}, \quad f_{\mu \nu}^{L / R}=$ $f_{\mu \nu}^{L / R a} T_{a}^{L / R}$, and $f_{\mu \nu}^{(\alpha)}=f_{\mu \nu}^{(\alpha) \alpha} T_{\alpha}$ with $T_{a}^{L, R}$ are the six generators of $S U(2)_{L, R}$, those embedded into the unbroken generators $T^{a}$.

The new covariant structure variable $f_{\mu \nu}^{ \pm}$and its automorphism partner transforms as gauge covariants under the local symmetry $\mathfrak{h} \in \mathcal{H}$

$$
\begin{aligned}
f_{\mu \nu}^{ \pm} & \rightarrow \mathfrak{h}(\Pi, \mathfrak{g}) f_{\mu \nu}^{ \pm} \mathfrak{h}^{-1}(\Pi, \mathfrak{g}), \\
f_{\mu \nu}^{(R) \pm} & \rightarrow \mathfrak{h}(\Pi, \mathfrak{g}) f_{\mu \nu}^{(R)^{ \pm}} \mathfrak{h}^{-1}(\Pi, \mathfrak{g})
\end{aligned}
$$

According to Eq. (B55), with the aid of the trace property, one finds that the gauge invariant field Lagrangian can be decomposed as

$$
\operatorname{Tr}\left(\overline{\mathrm{F}}_{\mu \nu} \overline{\mathrm{F}}^{\mu \nu}\right)=\operatorname{Tr}\left(f_{\mu \nu}^{+} f^{+\mu \nu}\right)+\operatorname{Tr}\left(f_{\mu \nu}^{-} f^{-\mu \nu}\right) .
$$

Thus, one obtains a building block as below 


$$
\operatorname{Tr}\left(f_{\mu \nu}^{-} f^{-\mu \nu}\right), \quad \operatorname{Tr}\left(f_{\mu \nu}^{+} f^{+\mu \nu}\right) .
$$

From the gauged version of Eq. (B26), i.e., (B97), one expand the $\mathcal{G}$ invariant strength tensor as below:

$$
\begin{aligned}
f_{\mu \nu} & =\Omega^{\dagger} \overline{\mathrm{F}}_{\mu \nu} \Omega=-i \Omega^{\dagger}\left[\mathbf{D}_{\mu}, \mathbf{D}_{\nu}\right] \Omega \\
& =\Omega^{\dagger} \mathbf{D}_{\mu}\left(\Omega \omega_{\nu}\right)-\Omega^{\dagger} \mathbf{D}_{\nu}\left(\Omega \omega_{\mu}\right) \\
& =i\left[\omega_{\mu}, \omega_{\nu}\right]+\partial_{\mu} \omega_{\nu}-\partial_{\nu} \omega_{\mu} \equiv \omega_{\mu \nu} \\
& =\nabla_{\mu} d_{\nu}-\nabla_{\nu} d_{\mu}+i\left[d_{\mu}, d_{\nu}\right]+e_{\mu \nu} \\
& \equiv d_{\mu \nu}+e_{\mu \nu},
\end{aligned}
$$

where $\Omega^{\dagger}=\Omega^{-1}$ and we have used the factor that $\left[\mathbf{D}_{\mu}, \mathbf{D}_{\nu}\right]=i \overline{\mathrm{F}}_{\mu \nu}$, and made the notations as below

$$
\begin{aligned}
\omega_{\mu \nu} & \equiv \partial_{\mu} \omega_{\nu}-\partial_{\nu} \omega_{\mu}+i\left[\omega_{\mu}, \omega_{\nu}\right], \\
d_{\mu \nu} & \equiv \nabla_{\mu} d_{\nu}-\nabla_{\nu} d_{\mu}+i\left[d_{\mu}, d_{\nu}\right], \\
e_{\mu \nu} & \equiv \partial_{\mu} e_{\nu}-\partial_{\nu} e_{\mu}+i\left[e_{\mu}, e_{\nu}\right], \\
\nabla_{\mu} d_{\nu} & \equiv \partial_{\mu} d_{\nu}+i\left[e_{\mu}, d_{\nu}\right], \\
\nabla_{\nu} d_{\mu} & \equiv \partial_{\nu} d_{\mu}+i\left[e_{\nu}, d_{\mu}\right],
\end{aligned}
$$

where $\omega_{\mu}, e_{\mu}$ are the Cartan connection of $\mathcal{G}$ and $\mathcal{H}$, respectively. $\omega_{\mu \nu}$ and $e_{\mu \nu}$ are the corresponding field strength or curvature of the $\omega_{\mu}$ and $e_{\mu}$, respectively. Note that there is an exchange symmetry between $d_{\mu}$ and $e_{\nu}$ in the above definition of $f_{\mu \nu}$, originating from $\omega_{\mu}=d_{\mu}+e_{\nu}$ so that we have

$$
\begin{aligned}
f_{\mu \nu} & =e_{\mu \nu}+d_{\mu \nu}-i\left[d_{\nu}, e_{\mu}\right]+i\left[d_{\mu}, e_{\nu}\right] \\
& =\nabla_{\mu} e_{\nu}-\nabla_{\nu} e_{\mu}+i\left[e_{\mu}, e_{\nu}\right]+d_{\mu \nu}
\end{aligned}
$$

where

$$
\begin{aligned}
& \nabla_{\mu} e_{\nu} \equiv \partial_{\mu} e_{\nu}+i\left[d_{\mu}, e_{\nu}\right], \\
& \nabla_{\nu} e_{\mu} \equiv \partial_{\nu} e_{\mu}+i\left[d_{\nu}, e_{\mu}\right] .
\end{aligned}
$$

By comparing the identities in Eq. (B62) with the decomposition of the gauge field in Eq. (B55), we find the relations between the gauge fields with the $e_{\mu \nu}$ and $d_{\mu}$ as below

$$
f_{\mu \nu}^{+} \equiv i\left[d_{\mu}, d_{\nu}\right]+e_{\mu \nu}, \quad f_{\mu \nu}^{-} \equiv 2 \nabla_{[\mu} d_{\nu]},
$$

where $\nabla_{[\mu} d_{\nu]}=\left(\nabla_{\mu} d_{\nu}-\nabla_{\nu} d_{\mu}\right) / 2$. By imposing the automorphism symmetry upon both side of the first equation one has

$$
f_{\mu \nu}^{+}=i\left[d_{\mu}, d_{\nu}\right]+e_{\mu \nu}^{(\mathcal{R})}
$$

Then one concludes that $e_{\mu \nu}^{(\mathcal{R})}=e_{\mu \nu}$. By substituting Eq. (B46) back into Eq. (B66), and by using Eqs. (B29) and (B52), the gauge fields can be reexpressed as

$$
\begin{aligned}
f_{\mu \nu}^{+} & =i\left[d_{\mu}, d_{\nu}\right]+2 \partial_{[\mu} e_{\nu]}+i\left[e_{\mu}, e_{\nu}\right] \\
& =\frac{1}{2}\left(\omega_{\mu \nu}+\omega_{\mu \nu}^{(\mathcal{R})}\right)=e_{\mu \nu}+\frac{1}{2}\left(d_{\mu \nu}+d_{\mu \nu}^{(\mathcal{R})}\right), \\
f_{\mu \nu}^{-} & =2 \partial_{[\mu} d_{\nu]}+2 i\left[e_{[\mu}, d_{\nu]}\right] \\
& =\frac{1}{2}\left(\omega_{\mu \nu}-\omega_{\mu \nu}^{(\mathcal{R})}\right)=\frac{1}{2}\left(d_{\mu \nu}-d_{\mu \nu}^{(\mathcal{R})}\right),
\end{aligned}
$$

where we have defined the field strength of MC one-form with aut parity even and aut partity odd as

$$
\begin{aligned}
\omega_{\mu \nu} & \equiv 2 \partial_{[\mu} \omega_{\nu]}+i\left[\omega_{\mu}, \omega_{\nu}\right], \\
\omega_{\mu \nu}^{(\mathcal{R})} & \equiv 2 \partial_{[\mu} \omega_{\nu]}^{(\mathcal{R})}+i\left[\omega_{\mu}^{(\mathcal{R})}, \omega_{\nu}^{(\mathcal{R})}\right] .
\end{aligned}
$$

These are nothing but the two-form gauge fields in the dynamical Maurer-Cartan equation as

$$
\begin{aligned}
f_{\omega} & =d \omega+\frac{1}{2} i[\omega, \omega]=f^{+}+f^{-}, \\
f_{\omega}^{(\mathcal{R})} & =d \omega^{(\mathcal{R})}+\frac{i}{2}\left[\omega^{(\mathcal{R})}, \omega^{(\mathcal{R})}\right]=f^{+}-f^{-} .
\end{aligned}
$$

Thus, in differential form, the two-form $f^{ \pm}$becomes

$$
f^{ \pm}=\frac{1}{2}\left(f_{\omega} \pm f_{\omega}^{(\mathcal{R})}\right),
$$

where $f^{+}$is aut-even while $f^{-}$is aut-odd. Therefore, as expected, one has two independent building blocks as

$$
\begin{aligned}
i\left[d_{\mu}, d_{\nu}\right] & =\frac{1}{2}\left(d_{\mu \nu}+d_{\mu \nu}^{(\mathcal{R})}\right) \equiv d_{\mu \nu}^{+}, \\
2 \partial_{[\mu} \nabla_{\nu]} & =\frac{1}{2}\left(d_{\mu \nu}-d_{\mu \nu}^{(\mathcal{R})}\right) \equiv d_{\mu \nu}^{-} .
\end{aligned}
$$

By substituting back into Eq. (B55), one has

$$
\begin{aligned}
f_{\mu \nu} & =i\left[d_{\mu}, d_{\nu}\right]+e_{\mu \nu}+2 \nabla_{[\mu} d_{\nu]}, \\
f_{\mu \nu}^{(\mathcal{R})} & =i\left[d_{\mu}, d_{\nu}\right]+e_{\mu \nu}-2 \nabla_{[\mu} d_{\nu]},
\end{aligned}
$$

which can be reorganized as

$$
\begin{gathered}
f_{\mu \nu}-e_{\mu \nu}=2 \nabla_{[\mu} d_{\nu]}+i\left[d_{\mu}, d_{\nu}\right], \\
f_{\mu \nu}^{(\mathcal{R})}-e_{\mu \nu}=-2 \nabla_{[\mu} d_{\nu]}+i\left[d_{\mu}, d_{\nu}\right] .
\end{gathered}
$$

To be brief, the CCWZ covariant building blocks

$$
d_{\mu}, e_{\mu \nu}
$$

can be reexpressed in terms of $\boldsymbol{\Sigma}$, which is manifestly invariant under global $\mathcal{G}$ 


$$
\begin{aligned}
d_{\mu}= & -\frac{i}{2} \Omega^{-1}\left(D_{\mu} \boldsymbol{\Sigma}\right) \Omega^{-1}=\frac{i}{2} \Omega\left(D_{\mu} \boldsymbol{\Sigma}\right)^{-1} \Omega \\
e_{\mu \nu}= & -\frac{i}{4} \Omega^{-1}\left(D_{\mu} \boldsymbol{\Sigma} D_{\nu} \boldsymbol{\Sigma}^{-1}-D_{\nu} \boldsymbol{\Sigma} D_{\mu} \boldsymbol{\Sigma}^{\dagger}\right) \Omega \\
& +\frac{1}{2}\left(\Omega^{-1} \overline{\mathrm{F}}_{\mu \nu} \Omega+\Omega \overline{\mathrm{F}}_{\mu \nu}^{(\mathcal{R})} \Omega^{-1}\right),
\end{aligned}
$$

where in a symmetric $\operatorname{coset} \mathcal{G} / \mathcal{H}$. Thus $e_{\mu \nu}$ and it is related to the unbroken sector field strength $f_{\mu \nu}^{+}$and the commutator of the $d_{\mu}$ field.

Therefore, in the end we have a set of covariant building blocks as

$$
\left\{d_{\mu}, f_{\mu \nu}^{ \pm}\right\} \quad \text { or } \quad\left\{d_{\mu}, f_{\mu \nu}^{-}, e_{\mu \nu}\right\}
$$

In summary, we have three independent building blocks of the effective Lagrangian in the CCWZ formalism as $d_{\mu}, e_{\mu \nu}$, and $\nabla_{\mu}$, since they are covariant variables, which satisfies the local transformation under $\mathcal{H}$,

$$
d_{\mu} \rightarrow \mathfrak{h} d_{\mu} \mathfrak{h}^{-1}, \quad e_{\mu \nu} \rightarrow \mathfrak{h} e_{\mu \nu} \mathfrak{h}^{\dagger}, \quad f_{\mu \nu}^{ \pm} \rightarrow \mathfrak{h} \nabla_{\mu} \mathfrak{h}^{\dagger} .
$$

For the $S O(6) / S O(5)$ case, one has

$$
\begin{aligned}
& e_{\mu \nu}=e_{\mu \nu}^{a L} T^{a L}+e_{\mu \nu}^{a R} T^{a R}+e_{\mu \nu}^{\alpha} T^{\alpha}, \\
& f_{\mu \nu}=f_{\mu \nu}^{a L} T^{a L}+f_{\mu \nu}^{a R} T^{a R}+f_{\mu \nu}^{\alpha} T^{\alpha},
\end{aligned}
$$

where two independent gauge invariant field strength tensors can be defined as

$$
\begin{aligned}
e_{\mu \nu}^{L, R a} & =\partial_{\mu} e_{\nu}^{L, R a}-\partial_{\nu} e_{\mu}^{L, R a}+i\left[e_{\mu}, e_{\nu}\right]^{L, R a}, \\
e_{\mu \nu}^{\alpha} & =\partial_{\mu} e_{\nu}^{\alpha}-\partial_{\nu} e_{\mu}^{\alpha}+i\left[e_{\mu}, e_{\nu}\right]^{\alpha} .
\end{aligned}
$$

Under the automorphism symmetry $\mathcal{R}$ in Eq. (B5), its representation in the coset $S O(6) / S O(5)$ is shown in Eq. (2.38). Because the broken generators $T^{\hat{a}}$ change sign, while the unbroken ones $T^{a}$ do not, thus one has

$$
\begin{array}{rlrl}
h^{\hat{a}} & \rightarrow-h^{\hat{a}}, & d_{\mu}^{\hat{a}} & \rightarrow-d_{\mu}^{\hat{a}}, \quad f_{\mu \nu}^{-\hat{a}} \rightarrow-f_{\mu \nu}^{-\hat{a}}, \\
e_{\mu \nu}^{a} & \rightarrow e_{\mu \nu}^{a}, \quad f_{\mu \nu}^{+a} \rightarrow f_{\mu \nu}^{+a} .
\end{array}
$$

If $e_{\mu \nu}^{\alpha}$ and $f_{\mu \nu}^{\alpha}$ are absent, i.e., $\mathbf{6}=(\mathbf{3}, 1)+(1, \mathbf{3}) \in$ $S U(2)_{L} \times S U(2)_{R} \subset S O(4)$, then under left-right parity symmetry $P_{L R}$ in Eq. (2.44), one has

$T^{\hat{a}}: h^{\hat{a}} \rightarrow-\eta^{\hat{a}} h^{\hat{a}}, \quad d_{\mu}^{\hat{a}} \rightarrow-\eta^{\hat{a}} d_{\mu}^{\hat{a}}, \quad f_{\mu \nu}^{-\hat{a}} \rightarrow-\eta^{\hat{a}} f_{\mu \nu}^{-\hat{a}}$, $T^{a}: e_{\mu \nu}^{L / R a} \rightarrow e_{\mu \nu}^{R / L a}, \quad f_{\mu \nu}^{+L / R a} \rightarrow f_{\mu \nu}^{+R / L a}$,

where $\eta^{\hat{a}}=(1,1,1,-1)^{T}$.

\section{External gauge symmetry: SM or beyond}

Once an external gauge symmetry $\mathcal{H}_{0} \subset \mathcal{G}$ is turned on, e.g., the SM electroweak group $\mathcal{H}_{0}=\mathcal{G}_{\mathrm{SM}}=S U(2)_{W} \times$ $U(1)_{Y}$, the ordinary derivative $\partial_{\mu}$ is promoted to be the covariant derivative $D_{\mu} \equiv \partial_{\mu}+i A_{\mu}$, where the external gauge fields $A_{\mu}=A_{\mu}^{\hat{a}} T^{\hat{a}}+A_{\mu}^{a} T^{a} \in \mathcal{G}$, i.e., still a general element of $\mathcal{G}$, transform under the local $\mathcal{H}_{0}$ transformation, namely

$$
A_{\mu} \rightarrow \mathfrak{g}\left(A_{\mu}-i \partial_{\mu}\right) \mathfrak{g}^{\dagger}, \quad F_{\mu \nu} \rightarrow \mathfrak{g} F_{\mu \nu} \mathfrak{g}^{\dagger},
$$

where $F_{\mu \nu} \equiv \partial_{\mu} A_{\nu}-\partial_{\nu} A_{\mu}+i\left[A_{\mu}, A_{\nu}\right]$ is the field strength of external gauge field $A_{\mu}$. Some of the above external gauge field source will become dynamical while the others will be turned off, i.e., by setting the others to zero. Explicitly, the dynamical part of $A_{\mu}$ will be those transforming under the local $\mathcal{H}_{0}=\mathcal{G}_{\mathrm{EW}}=S U(2)_{W} \times$ $U(1)_{Y} \subset \mathcal{H}$,

$$
A_{\mu}=\frac{g}{\sqrt{2}} W_{\mu}^{+} T_{L}^{+}+\frac{g}{\sqrt{2}} W_{\mu}^{-} T_{L}^{-}+g W_{\mu}^{3} T_{L}^{3}+g^{\prime} B_{\mu} Y,
$$

where $T_{L}^{ \pm}=T_{L}^{1} \pm i T_{L}^{2}$. The charged weak boson fields are defined as

$$
W_{\mu}^{ \pm}=\frac{1}{\sqrt{2}}\left(W_{\mu}^{1} \mp i W_{\mu}^{2}\right) .
$$

For SM, $A_{\mu}=g\left(W_{\mu}^{1} \sigma^{1}+W_{\mu}^{2} \sigma^{2}+W_{\mu}^{3} \sigma^{2}\right) / 2+g^{\prime} B_{\mu} Y$ with $Y=1 / 2, g, g^{\prime}$ are the $S U(2)_{L}$ and $U(1)$ gauge coupling, respectively. The neutral gauge boson fields are

$$
\begin{aligned}
g W_{\mu}^{3} T_{L}^{3}+g^{\prime} B_{\mu} Y & =g_{Z}\left(T^{3}-s_{W}^{2} Q\right) Z_{\mu}+e Q \gamma_{\mu}, \\
\left(\begin{array}{c}
W_{\mu}^{3} \\
B_{\mu}
\end{array}\right) & =\left(\begin{array}{cc}
c_{W} & s_{W} \\
-s_{W} & c_{W}
\end{array}\right)\left(\begin{array}{c}
Z_{\mu} \\
\gamma_{\mu}
\end{array}\right),
\end{aligned}
$$

where $\quad c_{W}=\cos \theta_{W} \equiv g / \sqrt{g^{2}+g^{\prime 2}}, \quad s_{W}=\sin \theta_{W} \equiv$ $g^{\prime} / \sqrt{g^{2}+g^{\prime 2}}$ denote the cosine and the since of the weak mixing angle, and $\tan \theta_{W}=g^{\prime} / g$ and $g, g^{\prime}$ are the gauge couplings of $S U(2)_{L}$ and $U(1)_{Y}$ in the SM. The commutator of the gauge field strength is

$$
\left[\mathbf{D}_{\mu}, \mathbf{D}_{\nu}\right]=i g \mathbf{W}_{\mu \nu}+i g^{\prime} \frac{1}{2} B_{\mu \nu} .
$$

Similarly, if one want to turn on the residual $\mathcal{H}$ invariant gauge source, e.g., $S U(2)_{R} \subset \mathcal{H}$, then one can include an additional term at a high-energy scale $f \neq v$.

$$
A_{\mu}=\frac{g_{R}}{\sqrt{2}} W_{R \mu}^{+} T_{R}^{+}+\frac{g_{R}}{\sqrt{2}} W_{R \mu}^{-} T_{R}^{-}+g_{R} Z_{R \mu} T_{R}^{3},
$$

where $T_{R}^{ \pm}=T_{R}^{1} \pm i T_{R}^{2}$ and generally, $g_{R} \neq g_{L}=g$. One may also denote respectively $W_{R}^{ \pm}, Z_{R}$ as $W^{\prime \pm}, Z^{\prime}$. While in 
the effective Lagrangian description, one may integrate out these heavy particle fields.

For mater field $\Phi$ which transforms as $\Phi \rightarrow \mathfrak{h}(\Pi, g) \Phi$, the covariant derivative is

$$
\nabla_{\mu} \Phi=\left(\partial_{\mu}+i e_{\mu}^{a} T^{a}\right) \Phi .
$$

Therefore, ordinary derivative $\partial_{\mu}$ can be promoted to be covariant derivative

$$
\nabla_{\mu} \equiv \partial_{\mu}+i e_{\mu},
$$

which is gauge invariant under $\mathcal{H}$.

One may also introduce the vector resonances $\rho^{ \pm}$. The strength of the external gauge fields transforms under the local symmetry $\mathcal{H}, \quad F_{\mu \nu}^{ \pm}(\Pi) \rightarrow \mathfrak{h}(\Pi, g) F_{\mu \nu}^{ \pm}(\Pi) \mathfrak{h}^{-1}(\Pi, g)$. $F_{\mu \nu}^{ \pm} \equiv \rho_{\mu \nu}^{ \pm}$, which takes even and odd $\mathcal{R}$-parity respectively, i.e., $\quad F_{\mu \nu}^{+} \equiv \rho_{\mu \nu}^{+a} T^{a} \in \operatorname{adj}(\mathcal{H}), \quad F_{\mu \nu}^{-} \equiv \rho_{\mu \nu}^{-\hat{a}} T^{\hat{a}} \in \mathcal{G} / \mathcal{H} \quad$ and transform under $\mathcal{G}$. Then kinetic and mass terms of the gauge field are

$$
\mathcal{L}_{F}=\frac{1}{2} \operatorname{Tr}\left[\nabla^{\mu} F_{\mu \sigma}^{ \pm} \nabla_{\nu} F^{ \pm \nu \sigma}\right]+\frac{1}{4} m_{ \pm}^{2} \operatorname{Tr}\left[F_{\mu \nu}^{ \pm} F^{ \pm \mu \nu}\right],
$$

where

$$
\nabla_{\mu} A_{\nu}=\partial_{\mu} A_{\nu}+i\left[e_{\mu}, A_{\nu}\right]
$$

One may also introduce the next order effective Lagrangian, by coupling the external gauge symmetry with the building blocks as

$$
\begin{aligned}
\mathcal{L}= & c_{g_{+}} \operatorname{Tr}\left(F_{\mu \nu}^{+}\left[d_{\mu}, d_{\nu}\right]\right)+c_{f_{+}} \operatorname{Tr}\left(F_{\mu \nu}^{+} f^{+\mu \nu}\right) \\
& +c_{f_{-}} \operatorname{Tr}\left(F_{\mu \nu}^{-} f^{-\mu \nu}\right),
\end{aligned}
$$

which is relevant to the $S$ parameter.

\section{Nonlinear chiral Lagrangian}

It is worth noticing that the GBs field $\Pi$ in Eq. (B1) enters in the effective Lagrangian only through its derivatives. The derivative of the $\Omega$ field can be expanded through the one-form $\omega_{\mu}$ as

$$
\begin{aligned}
\omega_{\mu}= & \sum_{n=1}^{\infty} \frac{(-i)^{n-1}}{n !}\left[\Pi,\left[\Pi, \ldots,\left[\Pi, \partial_{\mu} \Pi\right] \ldots\right]\right] \\
= & \partial_{\mu} \Pi-\frac{i}{2}\left[\Pi, \partial_{\mu} \Pi\right]-\frac{1}{6}\left[\Pi,\left[\Pi, \partial_{\mu} \Pi\right]\right] \\
& +\frac{i}{24}\left[\Pi,\left[\Pi,\left[\Pi, \partial_{\mu} \Pi\right]\right]\right]+\cdots,
\end{aligned}
$$

where $\left[\Pi, \partial_{\mu} \Pi\right]=\Pi \stackrel{\leftrightarrow}{\partial}_{\mu} \Pi$. In this case, the leading-order terms expanding the filed $d_{\mu}$ and $e_{\mu}$ are

$$
\begin{aligned}
& d_{\mu}=\partial_{\mu} \Pi-\frac{1}{6}\left[\Pi,\left[\Pi, \partial_{\mu} \Pi\right]\right]+\mathcal{O}\left(\Pi^{5}\right), \\
& e_{\mu}=-\frac{i}{2}\left[\Pi, \partial_{\mu} \Pi\right]+\frac{i}{24}\left[\Pi,\left[\Pi,\left[\Pi, \partial_{\mu} \Pi\right]\right]\right]+\mathcal{O}\left(\Pi^{6}\right) .
\end{aligned}
$$

When external gauge interactions $\overline{\mathrm{F}}_{\mu \nu}=F_{\mu \nu}^{a} T^{a}, \overline{\mathrm{A}}_{\mu}=$ $A_{\mu}^{a} T^{a}$ are turned on, i.e., $\partial_{\mu} \rightarrow D_{\mu}=\partial_{\mu}+i A_{\mu}^{a} T^{a}$, the Higgs will be interacting with gauge bosons too. Then, the covariant derivative of the $\Pi$ field is

$D_{\mu} \Pi^{\hat{a}}=\left(\partial_{\mu}+i A_{\mu}^{a} T^{a}\right) \Pi^{\hat{a}}=\partial_{\mu} \Pi^{\hat{a}}+i A_{\mu}^{a}\left(T^{a}\right)_{\hat{b}}^{\hat{a}} \Pi^{\hat{b}}$,

where for simplicity, one may restrict attention to the case in which $A_{\mu}$ belongs to $\mathcal{H}^{0} \subset \mathcal{H}$ is given by Eq. (B84),

$$
\begin{aligned}
\omega_{\mu} & \equiv-i \Omega^{\dagger} D_{\mu} \Omega=-i \Omega^{\dagger} \partial_{\mu} \Omega+\Omega^{\dagger} A_{\mu} \Omega \\
& =\bar{d}_{\mu}+\bar{e}_{\mu}+A_{\mu} .
\end{aligned}
$$

Thus, for the gauged version, one can redefine $e_{\mu}$ in a way as

$$
\bar{e}_{\mu}=e_{\mu}-A_{\mu}, \quad \bar{d}_{\mu}=d_{\mu}
$$

and the gauge field strength is

$$
\bar{e}_{\mu \nu}=-F_{\mu \nu}+e_{\mu \nu} .
$$

Under the automorphism symmetry $\mathcal{R}$ in Eq. (B5), we have

$$
\mathcal{R}\left(d_{\mu}^{\hat{a}}\right) \rightarrow-d_{\mu}^{\hat{a}}, \quad \mathcal{R}\left(e_{\mu}^{a}\right) \rightarrow e_{\mu}^{a} .
$$

By using the building blocks $d_{\mu}^{\hat{a}}$, the lowest order Lagrangian can be written as

$$
\begin{aligned}
\mathcal{L}_{d d}^{(2)} & =\frac{f^{2}}{2} d_{\mu}^{\hat{a}} d^{\hat{a} \mu}=\frac{f^{2}}{2}\left(\partial_{\mu} \Pi \partial^{\mu} \Pi+\frac{1}{3}\left(\Pi \stackrel{\leftrightarrow}{\partial}^{\mu} \Pi\right)\left(\Pi \stackrel{\leftrightarrow}{\partial}_{\mu} \Pi\right)+\cdots\right) \\
& =\frac{1}{2}\left(\partial_{\mu} \pi\right)^{2}+\frac{1}{3 f^{2}}\left(\pi \stackrel{\leftrightarrow}{\partial}^{\mu} \pi\right)^{\hat{a}}\left(\pi \stackrel{\leftrightarrow}{\partial}_{\mu} \pi\right)^{\hat{a}}+\mathcal{O}\left(\pi^{6}\right), \quad(\mathrm{B} 101)
\end{aligned}
$$

where $\Pi \stackrel{\leftrightarrow}{\partial}_{\mu} \Pi=\left[\Pi, \partial_{\mu} \Pi\right]$ and in the last equality we make the notation as $\Pi \equiv \pi / f$ and $\pi=h \Xi / 2$. In this case, phenomenologically, $\pi$ has an extra derivative selfinteractions, and interactions with Higgs $h$. In the presence of the external gauge field $A_{\mu}$, the gauge interactions are introduced with $\partial_{\mu} \rightarrow D_{\mu}$, and the second term involves interactions of two-derivative invariant operators with four Higgs doublet.

The next-to-leading-order Lagrangian with derivative up to the four-derivative for the gauge field $e_{\mu \nu}$ can be written as 


$$
\begin{aligned}
\mathcal{L}_{e e}^{(4)}= & \operatorname{Tr}\left[e_{\mu \nu} e^{\mu \nu}\right]=e_{\mu \nu}^{a} e^{a \mu \nu}=\left(\partial_{\mu} e_{\nu}-\partial_{\nu} e_{\mu}+i\left[e_{\mu}, e_{\nu}\right]\right)^{2} \\
= & -\frac{1}{4}\left(\partial_{\mu}\left(\left[\Pi, \partial_{\nu} \Pi\right]\right)-\partial_{\nu}\left(\left[\Pi, \partial_{\mu} \Pi\right]\right)\right. \\
& \left.+i\left[\left[\Pi, \partial_{\mu} \Pi\right],\left[\Pi, \partial_{\nu} \Pi\right]\right]\right)^{2} \\
= & -\frac{1}{4}\left(2\left[\partial_{\mu} \Pi, \partial_{\nu} \Pi\right]+i\left[\left[\Pi, \partial_{\mu} \Pi\right],\left[\Pi, \partial_{\nu} \Pi\right]\right]\right)^{2} \\
= & -\frac{1}{f^{4}}\left[\partial_{\mu} \pi, \partial_{\nu} \pi\right]^{2}+\mathcal{O}\left(\pi^{6}\right) \\
= & -\left(f^{a b e} f^{c d e}\right) \frac{1}{f^{4}} \partial_{\mu} \pi^{a} \partial_{\nu} \pi^{b} \partial^{\mu} \pi^{c} \partial^{\nu} \pi^{d}+\cdots . \quad(\mathrm{B} 102
\end{aligned}
$$

The interactions are suppressed by $f^{-4}$, while when the gauge interactions are turned on, the external gauge field $A_{\mu}$ is present, the first term involves interactions of fourderivative invariant operators with four Higgs doublet, which are enhanced with $g^{4}$.

Another next-to-leading-order Lagrangian with mixing between $e_{\mu}$ and $d_{\mu}$ fields are

$$
\begin{aligned}
\mathcal{L}_{d e}^{(4)}= & \operatorname{Tr}\left[\left[d_{\mu}, d_{\nu}\right] e^{\mu \nu}\right]=f^{a b c} d_{\mu}^{\hat{a}} d_{\nu}^{\hat{b}} e^{c \mu \nu} \\
= & -\frac{i}{2} f^{a b c}\left(\partial_{\mu} \Pi^{a}+\cdots\right)\left(\partial_{\nu} \Pi^{b}+\cdots\right) \\
& \times\left(2\left[\partial^{\mu} \Pi, \partial^{\nu} \Pi\right]^{c}+\cdots\right) \\
= & -\frac{i}{f^{4}} f^{a b c} f^{d e c} \partial_{\mu} \pi^{a} \partial_{\nu} \pi^{a} \partial^{\mu} \pi^{d} \partial^{\nu} \pi^{e}+\cdots
\end{aligned}
$$

Thus, these lowest order Lagrangians relating to the gauge field will appear at least at an order higher than $p^{4} / f^{4}$. When the gauge interactions are turned on, one must make the replacement for $e_{\mu \nu} \rightarrow e_{\mu \nu}-F_{\mu \nu}$ while keeping $d_{\mu}$ the same, then the interactions involving two NambuGoldstone bosons and two gauge fields will appear.

\section{Explicit expression of CCWZ}

\section{a. $d_{\mu}$ field}

In the NMCHM, the explicit expression of $d_{\mu}$ fields in the coset sector $S O(6) / S O(5)$ are

$$
\begin{aligned}
d_{\mu}^{1,2} & =\frac{g W_{\mu}^{1,2}}{\sqrt{2}} \cos \left(\frac{\psi}{f}\right) \sin \left(\frac{\phi}{f}\right), \\
d_{\mu}^{3} & =\frac{g W_{\mu}^{3}-g^{\prime} B_{\mu}}{\sqrt{2}} \cos \left(\frac{\psi}{f}\right) \sin \left(\frac{\phi}{f}\right), \\
d_{\mu}^{4} & =\frac{\sqrt{2}}{f}\left[\partial_{\mu} \phi \cos \left(\frac{\psi}{f}\right)-\partial_{\mu} \psi \sin \left(\frac{\psi}{f}\right) \sin \left(\frac{\phi}{f}\right)\right], \\
d_{\mu}^{5} & =\frac{\sqrt{2}}{f}\left[\partial_{\mu} \phi \sin \left(\frac{\psi}{f}\right)+\partial_{\mu} \psi \cos \left(\frac{\psi}{f}\right) \sin \left(\frac{\phi}{f}\right)\right] .
\end{aligned}
$$

\section{b. $d_{\mu, \nu}$ field}

From the $d_{\mu}$ fields above, one can obtain their anticommutators as

$$
d_{\mu, \nu}^{a} \equiv\left[d_{\mu}, d_{\nu}\right]^{a}
$$

The explicit expressions of $d_{\mu, \nu}^{a}$ in $(\mathbf{1}, \mathbf{3})+(\mathbf{3}, \mathbf{1})$ are given in

$$
\begin{aligned}
i\left(d_{\mu, \nu}^{L, 1}+d_{\mu, \nu}^{R, 1}\right)= & \frac{1}{2} g \cos ^{2}\left(\frac{\psi}{f}\right) \sin ^{2}\left(\frac{\phi}{f}\right)\left(W_{\nu}^{2} Z_{\mu}-W_{\mu}^{2} Z_{\nu}\right), \\
i\left(d_{\mu, \nu}^{L, 1}-d_{\mu, \nu}^{R, 1}\right)= & \frac{1}{f} g \cos ^{2}\left(\frac{\psi}{f}\right) \sin \left(\frac{\phi}{f}\right)\left(\left(\phi_{\mu} W_{\nu}^{1}-\phi_{\nu} W_{\mu}^{1}\right)\right. \\
& \left.+\tan \left(\frac{\psi}{f}\right) \sin \left(\frac{\phi}{f}\right)\left(\psi_{\nu} W_{\mu}^{1}-\psi_{\mu} W_{\nu}^{1}\right)\right), \\
i\left(d_{\mu, \nu}^{L, 2}+d_{\mu, \nu}^{R, 2}\right)= & -\frac{1}{2} g \cos ^{2}\left(\frac{\psi}{f}\right) \sin ^{2}\left(\frac{\phi}{f}\right)\left(W_{\nu}^{1} Z_{\mu}-W_{\mu}^{1} Z_{\nu}\right), \\
i\left(d_{\mu, \nu}^{L, 2}-d_{\mu, \nu}^{R, 2}\right)= & \frac{1}{f} g \cos ^{2}\left(\frac{\psi}{f}\right) \sin \left(\frac{\phi}{f}\right)\left(\left(\phi_{\mu} W_{\nu}^{2}-\phi_{\nu} W_{\mu}^{2}\right)\right. \\
& \left.+\tan \left(\frac{\psi}{f}\right) \sin \left(\frac{\phi}{f}\right)\left(\psi_{\nu} W_{\mu}^{2}-\psi_{\mu} W_{\nu}^{2}\right)\right), \\
i\left(d_{\mu, \nu}^{L, 3}+d_{\mu, \nu}^{R, 3}\right)= & \frac{1}{2} g^{2} \cos ^{2}\left(\frac{\psi}{f}\right) \sin 2\left(\frac{\phi}{f}\right)\left(W_{\mu}^{2} W_{\nu}^{1}-W_{\mu}^{1} W_{\nu}^{2}\right), \\
i\left(d_{\mu, \nu}^{L, 3}-d_{\mu, \nu}^{R, 3}\right)= & \frac{1}{f} \cos \left(\frac{\psi}{f}\right) \sin \left(\frac{\phi}{f}\right)\left(\left(\phi_{\nu} Z_{\mu}-\phi_{\mu} Z_{\nu}\right)\right. \\
& \left.+\tan \left(\frac{\psi}{f}\right) \sin \left(\frac{\phi}{f}\right)\left(\psi_{\mu} Z_{\nu}-\psi_{\nu} Z_{\mu}\right)\right) .
\end{aligned}
$$

From the above, it is obvious that the chiral sector is relatively suppressed with an order $1 / f$ comparing to the vector sector.

For those in $(\mathbf{2}, \mathbf{2})$ due to $T^{\alpha}$, we have

$$
\begin{aligned}
i d_{\mu, \nu}^{1}= & \frac{1}{\sqrt{2} f} g \cos \left(\frac{\psi}{f}\right) \sin \left(\frac{\phi}{f}\right)\left(\sin \left(\frac{\psi}{f}\right)\left(\phi_{\mu} W_{\nu}-\phi_{\nu} W_{\mu}\right)\right. \\
& \left.+\cos \left(\frac{\psi}{f}\right) \sin \left(\frac{\phi}{f}\right)\left(\psi_{\mu} W_{\nu}-\psi_{\nu} W_{\mu}\right)\right), \\
i d_{\mu, \nu}^{2}= & \frac{1}{\sqrt{2} f} g \cos \left(\frac{\psi}{f}\right) \sin \left(\frac{\phi}{f}\right)\left(\sin \left(\frac{\psi}{f}\right)\left(\phi_{\mu} W_{\nu}^{2}-\phi_{\nu} W_{\mu}^{2}\right)\right. \\
& \left.+\cos \left(\frac{\psi}{f}\right) \sin \left(\frac{\phi}{f}\right)\left(\psi_{\mu} W_{\nu}^{2}-\psi_{\nu} W_{\mu}^{2}\right)\right), \\
i d_{\mu, \nu}^{3}= & \frac{1}{\sqrt{2} f} \cos \left(\frac{\psi}{f}\right) \sin \left(\frac{\phi}{f}\right)\left(\sin \left(\frac{\psi}{f}\right)\left(\phi_{\mu} Z_{\nu}-\phi_{\nu} Z_{\mu}\right),\right. \\
& \left.+\cos \left(\frac{\psi}{f}\right) \sin \left(\frac{\phi}{f}\right)\left(\psi_{\mu} Z_{\nu}-\psi_{\nu} Z_{\mu}\right)\right) \\
i d_{\mu, \nu}^{4}= & \frac{\sqrt{2}}{f^{2}} \sin \left(\frac{\phi}{f}\right)\left(\psi_{\mu} \phi_{\nu}-\phi_{\mu} \psi_{\nu}\right) .
\end{aligned}
$$


Comparing to the leading order, the first three $d_{\mu, \nu}^{1,2,3}$ are suppressed by $1 / f$, while the fourth $d_{\mu, \nu}^{4}$ is suppressed by $1 / f^{2}$.

\section{c. $e_{\mu}$ field}

The $e_{\mu}$ fields in the unbroken $S O(5)$ sector, have 10 components, which can be decomposed into $\mathbf{1 0}=(\mathbf{3}, 1)+$ $(1, \mathbf{3})+(\mathbf{2}, \mathbf{2}) \in S U(2)_{L} \times S U(2)_{R} \simeq S O(4)$ components. The $(\mathbf{3}, 1)+(1, \mathbf{3})$ due to the generators $T_{L, R}^{a}$ gives

$e_{L \mu}^{1,2}=g W_{L \mu}^{1,2}-g W_{L \mu}^{1,2} \cos ^{2}\left(\frac{\psi}{f}\right) \sin ^{2}\left(\frac{\phi}{2 f}\right)$,

$e_{L \mu}^{3}=g W_{L \mu}^{3}-\cos ^{2}\left(\frac{\psi}{f}\right) \sin ^{2}\left(\frac{\phi}{2 f}\right)\left(g W_{L \mu}^{3}-g^{\prime} B_{\mu}\right) ;$

$e_{R \mu}^{1,2}=g W_{L \mu}^{1,2} \cos ^{2}\left(\frac{\psi}{f}\right) \sin ^{2}\left(\frac{\phi}{2 f}\right)$,

$e_{R \mu}^{3}=\cos ^{2}\left(\frac{\psi}{f}\right) \sin ^{2}\left(\frac{\phi}{2 f}\right)\left(g W_{L \mu}^{3}-g^{\prime} B_{\mu}\right) ;$

where hence and forth, we have made the abbreviation of the subscript $L$ for the $W$ bosons field, i.e., $W_{\mu}=W_{L \mu}$. The $(\mathbf{2 , 2})$ due to $T^{\alpha}$ gives

$$
\begin{aligned}
e_{\mu}^{1,2} & =-\frac{g W_{\mu}^{1,2}}{\sqrt{2}} \sin \left(\frac{2 \psi}{f}\right) \sin ^{2}\left(\frac{\phi}{2 f}\right), \\
e_{\mu}^{3} & =-\frac{1}{\sqrt{2}}\left(g W_{\mu}^{3}-g^{\prime} B_{\mu}\right) \sin \left(\frac{2 \psi}{f}\right) \sin ^{2}\left(\frac{\phi}{2 f}\right), \\
e_{\mu}^{4} & =\frac{\sqrt{2}}{f} \partial_{\mu} \psi\left[1-\cos \left(\frac{\phi}{f}\right)\right] .
\end{aligned}
$$

\section{d. $f_{\mu \nu}^{ \pm}$field}

In the Omega representation, the building blocks of the gauge field are not $F_{\mu \nu}$, but $f_{\mu \nu}^{ \pm}$as we will give the explicit expressions in the following.

According to the definitions in Eq. (B56), we have the gauge field $f_{\mu \nu}^{+}=\left(f_{\mu \nu}^{L, a}, f_{\mu \nu}^{R, a}, f_{\mu \nu}^{+, \alpha}\right)$ in the unbroken sector as

$$
\begin{aligned}
f_{\mu \nu}^{L, 1,2} & =g W_{\mu \nu}^{1,2}\left[1-\cos ^{2}\left(\frac{\psi}{f}\right) \sin ^{2}\left(\frac{\phi}{2 f}\right)\right], \\
f_{\mu \nu}^{L, 3} & =g W_{\mu \nu}^{3}-\cos ^{2}\left(\frac{\psi}{f}\right) \sin ^{2}\left(\frac{\phi}{2 f}\right)\left(g W_{\mu \nu}^{3}-g^{\prime} B_{\mu \nu}\right), \\
f_{\mu \nu}^{R, 1,2} & =g W_{\mu \nu}^{1,2} \cos ^{2}\left(\frac{\psi}{f}\right) \sin ^{2}\left(\frac{\phi}{2 f}\right), \\
f_{\mu \nu}^{R, 3} & =g^{\prime} B_{\mu \nu}+\cos ^{2}\left(\frac{\psi}{f}\right) \sin ^{2}\left(\frac{\phi}{2 f}\right)\left(g W_{\mu \nu}^{3}-g^{\prime} B_{\mu \nu}\right),
\end{aligned}
$$

which leads to an equivalent linear-independent combination as

$$
\begin{aligned}
f_{\mu \nu}^{L, 1,2}+f_{\mu \nu}^{R, 1,2} & =g W_{\mu \nu}^{1,2}, \\
f_{\mu \nu}^{L, 1,2}-f_{\mu \nu}^{R, 1,2} & =g W_{\mu \nu}^{1,2}\left[1-2 \cos ^{2}\left(\frac{\psi}{f}\right) \sin ^{2}\left(\frac{\phi}{2 f}\right)\right], \\
f_{\mu \nu}^{L, 3}+f_{\mu \nu}^{R, 3} & =g W_{\mu \nu}^{3}+g^{\prime} B_{\mu \nu} \equiv A_{\mu \nu}, \\
f_{\mu \nu}^{L, 3}-f_{\mu \nu}^{R, 3} & =Z_{\mu \nu}\left[1-2 \cos ^{2}\left(\frac{\psi}{f}\right) \sin ^{2}\left(\frac{\phi}{2 f}\right)\right],
\end{aligned}
$$

where $Z_{\mu \nu} \equiv g W_{\mu \nu}^{3}-g^{\prime} B_{\mu \nu}$. It is obvious that the vector $f_{\mu \nu}^{L, 3}+f_{\mu \nu}^{R, 3}$ is nothing but the gauge stress tensor for photon fields in the SM. The components in the $(\mathbf{2}, \mathbf{2})$ are

$f_{\mu \nu}^{+, 1}=-\frac{g}{\sqrt{2}} W_{\mu \nu}^{1} \sin \left(\frac{2 \psi}{f}\right) \sin ^{2}\left(\frac{\phi}{2 f}\right)$,

$f_{\mu \nu}^{+, 2}=-\frac{g}{\sqrt{2}} W_{\mu \nu}^{2} \sin \left(\frac{2 \psi}{f}\right) \sin ^{2}\left(\frac{\phi}{2 f}\right)$,

$f_{\mu \nu}^{+, 3}=-\frac{1}{\sqrt{2}}\left(g W_{\mu \nu}^{3}-g^{\prime} B_{\mu \nu}\right) \sin \left(\frac{2 \psi}{f}\right) \sin ^{2}\left(\frac{\phi}{2 f}\right)$,

$f_{\mu \nu}^{+, 4}=0$.

For the gauge field $f_{\mu \nu}^{-}$in the coset $S O(6) / S O(5)$ sector,

$f_{\mu \nu}^{-, 1}=\frac{g}{\sqrt{2}} W_{\mu \nu}^{1} \cos \left(\frac{\psi}{f}\right) \sin \left(\frac{\phi}{f}\right)$,

$f_{\mu \nu}^{-2}=\frac{g}{\sqrt{2}} W_{\mu \nu}^{2} \cos \left(\frac{\psi}{f}\right) \sin \left(\frac{\phi}{f}\right)$,

$f_{\mu \nu}^{-3}=\frac{1}{\sqrt{2}}\left(g W_{\mu \nu}^{3}-B_{\mu \nu} g^{\prime}\right) \cos \left(\frac{\psi}{f}\right) \sin \left(\frac{\phi}{f}\right)$,

$f_{\mu \nu}^{-, 4}=0, \quad f_{\mu \nu}^{-5}=0$,

where $W_{\mu \nu}^{a}$ and $B_{\mu \nu}$ are the gauge field stress tensors for $S U(2)_{L}$ and $U(1)_{L}$ symmetry

$$
\begin{aligned}
W_{\mu \nu}^{a} & =\partial_{\mu} W_{\nu}^{a}-\partial_{\nu} W_{\mu}^{b}-g \epsilon^{a b c} W_{\mu}^{b} W_{\nu}^{c}, \\
B_{\mu \nu} & =\partial_{\mu} B_{\nu}-\partial_{\nu} B_{\mu},
\end{aligned}
$$

since we have defined $F_{\mu \nu}=\partial_{\mu} A_{\nu}-\partial_{\nu} A_{\mu}+i\left[A_{\mu}, A_{\nu}\right]$.

\section{e. $e_{\mu \nu}$ field}

In NMCHM, the gauge field stress tensor in Eq. (B29) becomes

$$
e_{\mu \nu}=e_{\mu \nu}^{L}+e_{\mu \nu}^{R}+e_{\mu \nu}^{(\alpha)},
$$

where $e_{\mu \nu}^{L / R}=e_{\mu \nu}^{L / R, a} T_{a}^{L / R}$ and $e_{\mu \nu}^{(\alpha)}=e_{\mu \nu}^{(\alpha) \alpha} T_{\alpha}$.

The $e_{\mu \nu}$ can be computed through separating into the two sector as 


$$
e_{\mu \nu}=2 \partial_{[\mu} e_{\nu]},-i e_{\mu, \nu},
$$

where the Abelian sector of the fields is

$$
\partial_{\mu} e_{\nu}-\partial_{\nu} e_{\mu} \equiv 2 \partial_{[\mu} e_{\nu]}
$$

while the non-Abelian sector is

$$
e_{\mu, \nu} \equiv\left[e_{\mu}, e_{\nu}\right]
$$

The explicit expression in the NMCHM turns out to be

$$
\begin{aligned}
& i\left(e_{\mu, \nu}^{L, 1}+e_{\mu, \nu}^{R, 1}\right)=2 g \cos ^{2}\left(\frac{\psi}{f}\right) \sin ^{4}\left(\frac{\phi}{2 f}\right)\left(W_{\nu}^{2} Z_{\mu}-W_{\mu}^{2} Z_{\nu}\right), \\
& i\left(e_{\mu, \nu}^{L, 1}-e_{\mu, \nu}^{R, 1}\right)=\frac{2 g}{f} \sin \left(\frac{2 \psi}{f}\right) \sin ^{4}\left(\frac{\phi}{2 f}\right)\left(\psi_{\nu} W_{\mu}^{1}-\psi_{\mu} W_{\nu}^{1}\right), \\
& i\left(e_{\mu, \nu}^{L, 2}+e_{\mu, \nu}^{R, 2}\right)=2 g \cos ^{2}\left(\frac{\psi}{f}\right) \sin ^{4}\left(\frac{\phi}{2 f}\right)\left(W_{\mu}^{1} Z_{\nu}-W_{\nu}^{1} Z_{\mu}\right), \\
& i\left(e_{\mu, \nu}^{L, 2}-e_{\mu, \nu}^{R, 2}\right)=\frac{2 g}{f} \sin \left(\frac{2 \psi}{f}\right) \sin ^{4}\left(\frac{\phi}{2 f}\right)\left(\psi_{\nu} W_{\mu}^{2}-\psi_{\mu} W_{\nu}^{2}\right), \\
& i\left(e_{\mu, \nu}^{L, 3}+e_{\mu, \nu}^{R, 3}\right)=2 g^{2} \cos ^{2}\left(\frac{\psi}{f}\right) \sin ^{4}\left(\frac{\phi}{2 f}\right)\left(W_{\mu}^{2} W_{\nu}^{1}-W_{\mu}^{1} W_{\nu}^{2}\right), \\
& i\left(e_{\mu, \nu}^{L, 3}-e_{\mu, \nu}^{R, 3}\right)=\frac{2}{f} \sin \left(\frac{2 \psi}{f}\right) \sin ^{4}\left(\frac{\phi}{2 f}\right)\left(\psi_{\nu} Z_{\mu}-\psi_{\mu} Z_{\nu}\right) .
\end{aligned}
$$

The situation is similar to what has happened in $d_{\mu, \nu}$, the vector contribution is larger than the chiral contribution. For $e_{\mu, \nu}$ in $(\mathbf{2}, \mathbf{2})$, they are

$$
\begin{aligned}
i e_{\mu, \nu}^{1} & =\frac{2 \sqrt{2}}{f} g \cos ^{2}\left(\frac{\psi}{f}\right) \sin ^{4}\left(\frac{\phi}{2 f}\right)\left(\psi_{\mu} W_{\nu}^{1}-\psi_{\nu} W_{\mu}^{1}\right), \\
i e_{\mu, \nu}^{2} & =\frac{2 \sqrt{2}}{f} g \cos ^{2}\left(\frac{\psi}{f}\right) \sin ^{4}\left(\frac{\phi}{2 f}\right)\left(\psi_{\mu} W_{\nu}^{2}-\psi_{\nu} W_{\mu}^{2}\right), \\
i e_{\mu, \nu}^{3} & =\frac{2 \sqrt{2}}{f} \cos ^{2}\left(\frac{\psi}{f}\right) \sin ^{4}\left(\frac{\phi}{2 f}\right)\left(\psi_{\mu} Z_{\nu}-\psi_{\nu} Z_{\mu}\right), \\
e_{\mu, \nu}^{4} & =0
\end{aligned}
$$

We have also calculated all of the commutative parts of $e_{\mu \nu}$ in Eq. (B117),

$$
\begin{aligned}
\partial_{[\mu} e_{\nu]}^{A, 1,2}= & g \cos ^{2}\left(\frac{\psi}{f}\right) \sin ^{2}\left(\frac{\phi}{2 f}\right)\left(\partial_{\nu} W_{\mu}^{1,2}-\partial_{\mu} W_{\nu}^{1,2}\right) \\
& +\frac{1}{2 f} g\left(\cos ^{2}\left(\frac{\psi}{f}\right) \sin \left(\frac{\phi}{f}\right)\left(\phi_{\nu} W_{\mu}^{1,2}-\phi_{\mu} W_{\nu}^{1,2}\right)\right. \\
& \left.+2 \sin \left(\frac{2 \psi}{f}\right) \sin ^{2}\left(\frac{\phi}{2 f}\right)\left(\psi_{\mu} W_{\nu}^{1,2}-\psi_{\nu} W_{\mu}^{1,2}\right)\right) \\
\partial_{[\mu} e_{\nu]}^{A, 3}= & \cos ^{2}\left(\frac{\psi}{f}\right) \sin ^{2}\left(\frac{\phi}{2 f}\right)\left(\partial_{\nu} Z_{\mu}-\partial_{\mu} Z_{\nu}\right) \\
& +\frac{1}{2 f}\left(\cos ^{2}\left(\frac{\psi}{f}\right) \sin \left(\frac{\phi}{f}\right)\left(\phi_{\nu} Z_{\mu}-\phi_{\mu} Z_{\nu}\right)\right. \\
& \left.+2 \sin \left(\frac{2 \psi}{f}\right) \sin ^{2}\left(\frac{\phi}{2 f}\right)\left(\psi_{\mu} Z_{\nu}-\psi_{\nu} Z_{\mu}\right)\right)
\end{aligned}
$$$$
\partial_{[\mu} e_{\nu]}^{V, 1,2,3}=0
$$

where we have made the recombination $\partial_{[\mu} e_{\nu]}^{V / A, a} \equiv$ $\partial_{[\mu} e_{\nu]}^{L, a} \pm \partial_{[\mu} e_{\nu]}^{R a}$ with $a=1,2,3$.

For the $(\mathbf{2}, \mathbf{2})$, one has

$$
\begin{aligned}
\partial_{[\mu} e_{\nu]}^{1,2}= & \frac{g}{2 \sqrt{2}} \sin \left(\frac{2 \psi}{f}\right) \sin ^{2}\left(\frac{\phi}{2 f}\right)\left(\partial_{\nu} W_{\mu}^{1,2}-\partial_{\mu} W_{\nu}^{1,2}\right) \\
& +\frac{1}{2 \sqrt{2} f} g\left(\frac{1}{2} \sin \left(\frac{2 \psi}{f}\right) \sin \left(\frac{\phi}{f}\right)\left(\phi_{\nu} W_{\mu}^{1,2}-\phi_{\mu} W_{\nu}^{1,2}\right)\right. \\
& \left.+2 \cos \left(\frac{2 \psi}{f}\right) \sin ^{2}\left(\frac{\phi}{2 f}\right)\left(\psi_{\nu} W_{\mu}^{1,2}-\psi_{\mu} W_{\nu}^{1,2}\right)\right), \\
\partial_{[\mu} e_{\nu]}^{3}= & \frac{1}{2 \sqrt{2}} \sin \left(\frac{2 \psi}{f}\right) \sin ^{2}\left(\frac{\phi}{2 f}\right)\left(\partial_{\nu} Z_{\mu}-\partial_{\mu} Z_{\nu}\right) \\
& +\frac{1}{2 \sqrt{2} f}\left(\frac{1}{2} \sin \left(\frac{2 \psi}{f}\right) \sin \left(\frac{\phi}{f}\right)\left(\phi_{\nu} Z_{\mu}-\phi_{\mu} Z_{\nu}\right)\right. \\
& \left.+2 \cos \left(\frac{2 \psi}{f}\right) \sin ^{2}\left(\frac{\phi}{2 f}\right)\left(\psi_{\nu} Z_{\mu}-\psi_{\mu} Z_{\nu}\right)\right)
\end{aligned}
$$$$
\partial_{[\mu} e_{\nu]}^{4}=\frac{1}{\sqrt{2} f^{2}} \sin \left(\frac{\phi}{f}\right)\left(\phi_{\mu} \psi_{\nu}-\psi_{\mu} \phi_{\nu}\right) \text {. }
$$

According to Eq. (B66), the field strength $e_{\mu \nu}$ is also related to the unbroken sector $f_{\mu \nu}^{+}$and $d_{\mu}$ field through an identity

$$
e_{\mu \nu}=f_{\mu \nu}^{+}-i\left[d_{\mu}, d_{\nu}\right]=f_{\mu \nu}^{+}-i d_{\mu, \nu}
$$

Thus, they are not linear independent of $f_{\mu \nu}^{+}$. Thus, we can either use $e_{\mu \nu}$ or $f_{\mu \nu}^{+}$as the building block. The easiest but nontrivial check to determine the sign is through $\alpha=4$ components, which leads to

$$
e_{\mu \nu}^{4}=\partial_{\mu} e_{\nu}^{4}-\partial_{\nu} e_{\mu}^{4}=2 \partial_{[\mu} e_{\nu]}^{4}=-i d_{\mu, \nu}^{4}
$$

since $f_{\mu \nu}^{+, 4}=0$. 


\section{High-energy effective operators in literature}

In literature $[19,49,50,73]$, there are a set of high-energy effective operators defined in studying MCHM. In this section, we compute them explicitly in the NMCHM and also we make comparison with our results in Sec. IV.

At $p^{2}$ order, there are two $C P$-even operators, either keeping or breaking the custodial symmetry, and one $C P$-odd operator can be defined, respectively, as

$$
\begin{aligned}
& \mathcal{O}_{C}=f^{2} \operatorname{Tr}\left(d_{\mu} d^{\mu}\right) \propto \overline{\mathcal{L}}_{C}, \\
& \mathcal{O}_{T}=f^{2}\left[\operatorname{Tr}\left(d_{\mu} \chi\right)\right]^{2} \propto \overline{\mathcal{L}}_{T}, \\
& \mathcal{O}_{\bar{T}}=f^{2} \operatorname{Tr}\left[\left(\nabla_{\mu} d^{\mu}\right) \chi\right] \propto \overline{\mathcal{L}}_{\tilde{T}},
\end{aligned}
$$

where the factor $1 / 4$ in front of $\mathcal{L}_{C}$ is chosen for the canonical normalization of the kinetic term of the scalar in the $\Omega$ parametrization, as will be clear in Eq. (5.3). Both operators $\mathcal{O}_{T, \tilde{T}}$ are the custodial breaking terms that can contribute to the $T$ parameter.

At $p^{4}$ order there are seven $C P$-even operators constructed from the building blocks as below $[19,49,50]$,

$$
\begin{aligned}
\mathcal{O}_{1} & =\left[\operatorname{Tr}\left(d_{\mu} d^{\mu}\right)\right]^{2} \propto \overline{\mathcal{L}}_{4}, \\
\mathcal{O}_{2} & =\operatorname{Tr}\left(d_{\mu} d_{\nu}\right) \operatorname{Tr}\left(d^{\mu} d^{\nu}\right) \propto \overline{\mathcal{L}}_{5}, \\
\mathcal{O}_{3} & =\operatorname{Tr}\left[\left(e_{\mu \nu}^{L}\right)^{2}-\left(e_{\mu \nu}^{R}\right)^{2}\right], \\
\mathcal{O}_{4}^{ \pm} & =\operatorname{Tr}\left[\left(f_{\mu \nu}^{L} \pm f_{\mu \nu}^{R}\right) i\left[d^{\mu}, d^{\nu}\right]\right], \\
\mathcal{O}_{5}^{+} & =\operatorname{Tr}\left[\left(f_{\mu \nu}^{-}\right)^{2}\right] \propto \overline{\mathcal{L}}_{f}-\overline{\mathcal{L}}_{f \mathbf{\Sigma}}, \\
\mathcal{O}_{5}^{-} & =\operatorname{Tr}\left[\left(f_{\mu \nu}^{L}\right)^{2}-\left(f_{\mu \nu}^{R}\right)^{2}\right],
\end{aligned}
$$

where we have used Eq. (B66) and used the fact that $d_{\mu}^{(\mathcal{R})}=-d_{\mu}$, which is not independent of $d_{\mu}$. Therefore, $\mathcal{O}_{4}^{ \pm}$ can be viewed as a linear combination of $\overline{\mathcal{L}}_{2,3}$ with its own automorphism partner, and $\mathcal{O}_{5}^{ \pm}$is a linear combination of $\overline{\mathcal{L}}_{f}$ and $\overline{\mathcal{L}}_{f \boldsymbol{\Sigma}}$. Note that $f=(w, b), \mathcal{O}_{4}^{ \pm}(f)$ includes a set of four operators, so does $\mathcal{O}_{5}^{ \pm}(f)$. Later on, as neglected in Eq. 459, we have also calculated $\mathcal{O}_{3}$ and find the exact relation as below $\mathcal{O}_{3}=\mathcal{O}_{5}^{-}-2 \mathcal{O}_{4}^{-}$. Thus, $\mathcal{O}_{3}$ is not a linearly independent operator.

The first seven $p^{4}$ operators defined in Eq. (B126) can be expressed in an EW chiral Lagrangian as

$$
\begin{aligned}
\mathcal{O}_{k}= & -4 \mathcal{L}_{W}-4 \mathcal{L}_{B}, \\
\mathcal{O}_{1}= & \cos ^{4}\left(\frac{\psi}{f}\right) \sin ^{4}\left(\frac{\phi}{f}\right) \mathcal{L}_{6}-4 \cos ^{2}\left(\frac{\psi}{f}\right) \sin ^{2}\left(\frac{\phi}{f}\right) \mathcal{L}_{20}^{(\phi)} \\
& +4 \mathcal{L}_{D \phi}-4 \cos ^{2}\left(\frac{\psi}{f}\right) \sin ^{4}\left(\frac{\phi}{f}\right) \mathcal{L}_{20}^{(\psi)}+4 \sin ^{4}\left(\frac{\phi}{f}\right) \mathcal{L}_{D \psi}, \\
\mathcal{O}_{2}= & \cos ^{4}\left(\frac{\psi}{f}\right) \sin ^{4}\left(\frac{\phi}{f}\right) \mathcal{L}_{11}-4 \cos ^{2}\left(\frac{\psi}{f}\right) \sin ^{2}\left(\frac{\phi}{f}\right) \mathcal{L}_{8}^{(\phi)} \\
& +4 \mathcal{L}_{D \phi}-4 \cos ^{2}\left(\frac{\psi}{f}\right) \sin ^{4}\left(\frac{\phi}{f}\right) \mathcal{L}_{8}^{(\psi)}+4 \sin ^{4}\left(\frac{\phi}{f}\right) \mathcal{L}_{D \psi},
\end{aligned}
$$

$$
\begin{aligned}
& \mathcal{O}_{4}^{+}=-\frac{1}{4} \cos ^{2}\left(\frac{\psi}{f}\right) \sin ^{2}\left(\frac{\phi}{f}\right)\left(\mathcal{L}_{2}+2 \mathcal{L}_{3}\right) \\
& +\cos ^{2}\left(\frac{\psi}{f}\right) \sin \left(\frac{\phi}{f}\right) \\
& \times\left(1-2 \cos ^{2}\left(\frac{\psi}{f}\right) \sin ^{2}\left(\frac{\phi}{2 f}\right)\right)\left(\mathcal{L}_{4}^{(\phi)}-2 \mathcal{L}_{5}^{(\phi)}\right) \\
& -\sin \left(\frac{\psi}{f}\right) \cos \left(\frac{\psi}{f}\right) \sin ^{2}\left(\frac{\phi}{f}\right) \\
& \times\left(1-2 \cos ^{2}\left(\frac{\psi}{f}\right) \sin ^{2}\left(\frac{\phi}{2 f}\right)\right)\left(\mathcal{L}_{4}^{(\psi)}-2 \mathcal{L}_{5}^{(\psi)}\right), \\
& \mathcal{O}_{4}^{-}=\frac{1}{4} \cos ^{2}\left(\frac{\psi}{f}\right) \sin ^{2}\left(\frac{\phi}{f}\right)\left(1-2 \cos ^{2}\left(\frac{\psi}{f}\right) \sin ^{2}\left(\frac{\phi}{2 f}\right)\right) \\
& \times\left(\mathcal{L}_{2}-2 \mathcal{L}_{3}\right)-\cos ^{2}\left(\frac{\psi}{f}\right) \sin \left(\frac{\phi}{f}\right)\left(\mathcal{L}_{4}^{(\phi)}+2 \mathcal{L}_{5}^{(\phi)}\right) \\
& +\frac{1}{2} \sin \left(\frac{2 \psi}{f}\right) \sin ^{2}\left(\frac{\phi}{f}\right)\left(\mathcal{L}_{4}^{(\psi)}+2 \mathcal{L}_{5}^{(\psi)}\right), \\
& \mathcal{O}_{5}^{+}=-\cos ^{2}\left(\frac{\psi}{f}\right) \sin ^{2}\left(\frac{\phi}{f}\right)\left(\mathcal{L}_{1}+2 \mathcal{L}_{B}+2 \mathcal{L}_{W}\right), \\
& \mathcal{O}_{5}^{-}=4\left(1-2 \cos ^{2}\left(\frac{\psi}{f}\right) \sin ^{2}\left(\frac{\phi}{2 f}\right)\right)\left(\mathcal{L}_{B}-\mathcal{L}_{W}\right),
\end{aligned}
$$

where the low-energy effective Lagrangian $\mathcal{L}_{n}$ is defined in Eq. (C11), and $\mathcal{L}_{n}^{(\phi)}$ etc. follows the replacing rules in Eq. (4.4). The results are consistent with Eq. (A.34) in Ref. [49], after combining the known matching results in Eq. (4.3).

It is also worth noticing that the first two operators can also be reexpressed without expanding the square as

$$
\begin{aligned}
\mathcal{O}_{1}= & \frac{16}{f^{4}}\left[\mathcal{L}_{\phi}+\sin ^{2}\left(\frac{\phi}{f}\right) \mathcal{L}_{\psi}+\frac{1}{\xi} \cos ^{2}\left(\frac{\psi}{f}\right) \sin ^{2}\left(\frac{\phi}{f}\right) \mathcal{L}_{C}\right]^{2}, \\
\mathcal{O}_{2}= & \frac{16}{f^{4}}\left[\frac{1}{2} \partial_{\mu} \phi \partial_{\nu} \phi+\sin ^{2}\left(\frac{\phi}{f}\right) \frac{1}{2} \partial_{\mu} \psi \partial_{\nu} \psi+\frac{f^{2}}{8} \sin ^{2}\left(\frac{\psi}{f}\right)\right. \\
& \left.\times \cos ^{2}\left(\frac{\psi}{f}\right)\left[g^{2}\left(W_{\mu}^{1} W_{\nu}^{1}+W_{\mu}^{2} W_{\nu}^{2}\right)+Z_{\mu} Z_{\nu}\right]\right]^{2} .
\end{aligned}
$$

Thus, when $\psi=0$, it is also consistent with Eq. (30) in Ref. [50], by substituting back the EW chiral Lagrangian in Eq. (C11) and keeping the implicit form.

Similarly, five of the next-to-leading-order $C P$-odd operators can also be constructed from those as below $[19,73]$

$$
\begin{aligned}
& O_{6}^{ \pm}=2 \operatorname{Tr}\left[\left(\tilde{f}_{\mu \nu}^{L} \pm \tilde{f}_{\mu \nu}^{R}\right) i\left[d^{\mu}, d^{\nu}\right]\right] \propto \tilde{\mathcal{O}}_{4}^{ \pm}, \\
& O_{7}^{+}=2 \operatorname{Tr}\left(f_{\mu \nu}^{-} \tilde{f}^{\mu \nu-}\right) \propto \overline{\mathcal{L}}_{\tilde{f}}-\overline{\mathcal{L}}_{\tilde{f} \boldsymbol{\Sigma}}, \\
& O_{7}^{-}=2 \operatorname{Tr}\left(f_{\mu \nu}^{L} \tilde{f}^{L \mu \nu}-f_{\mu \nu}^{R} \tilde{f}^{R \mu \nu}\right), \\
& \mathcal{O}_{3}^{+}=\operatorname{Tr}\left[\left(e_{\mu \nu}^{L}\right)^{2}+\left(e_{\mu \nu}^{R}\right)^{2}\right],
\end{aligned}
$$


where $\tilde{f}_{\mu \nu}^{ \pm}=\epsilon_{\mu \nu \rho \sigma} f^{\rho \sigma \pm} / 2$ is a dual antisymmetric tensor. The $\mathcal{O}_{3}^{+}$can contributes to the $S$ parameter. This will become clear by transforming the basis into the SMEFT up to dimensionalsix operators.

To match with part of our basis in the $\Omega$ parametrization in Eqs. (3.15) and (3.17), with the building block $f_{\mu \nu}^{ \pm}$, we speculate that the more natural definitions of the seven effective $C P$-even and five $C P$-odd operators such as $\mathcal{O}_{3}$ etc. in Eq. (B126) and (B129), might be defined as below,

$$
\begin{aligned}
\mathcal{O}_{1} & =\left[\operatorname{Tr}\left(d_{\mu} d^{\mu}\right)\right]^{2} \propto \overline{\mathcal{L}}_{4}, \\
\mathcal{O}_{2} & =\operatorname{Tr}\left(d_{\mu} d_{\nu}\right) \operatorname{Tr}\left(d^{\mu} d^{\nu}\right) \propto \overline{\mathcal{L}}_{5}, \\
\mathcal{O}_{3} & \equiv \operatorname{Tr}\left(d_{\mu \nu}^{+} d^{+\mu \nu}\right) \propto \overline{\mathcal{L}}_{7,8}, \\
\mathcal{O}_{4}^{ \pm} & \equiv \operatorname{Tr}\left(d_{\mu \nu}^{+} f^{ \pm \mu \nu}\right) \propto \overline{\mathcal{L}}_{2,3} \pm \overline{\mathcal{L}}_{2,3}^{(\mathcal{R})}, \\
\mathcal{O}_{5}^{\mp} & \equiv \operatorname{Tr}\left(f_{\mu \nu}^{ \pm} f^{\mu \nu-}\right) \propto \overline{\mathcal{L}}_{f} \pm \overline{\mathcal{L}}_{f \mathbf{\Sigma}}, \\
O_{6}^{ \pm} & \equiv \operatorname{Tr}\left(d_{\mu \nu}^{+} \tilde{f}^{ \pm \mu \nu}\right) \propto \tilde{\mathcal{L}}_{2,3} \pm \tilde{\mathcal{L}}_{2,3}^{(\mathcal{R})}, \\
O_{7}^{\mp} & \equiv \operatorname{Tr}\left(f_{\mu \nu}^{ \pm} \tilde{f}^{\mu \nu-}\right) \propto \overline{\mathcal{L}}_{\tilde{f}} \pm \overline{\mathcal{L}}_{\tilde{f} \mathbf{\Sigma}}, \\
\mathcal{O}_{3}^{+} & =\operatorname{Tr}\left(d_{\mu \nu}^{+} \tilde{d}^{\mu \nu+}\right) \propto \overline{\mathcal{L}}_{\tilde{3}},
\end{aligned}
$$

where we have used Eq. (B66) so that $d_{\mu \nu}^{+}=f_{\mu \nu}^{+}-e_{\mu \nu}$, and $d_{\mu \nu}^{-}=f_{\mu \nu}^{-}$. It is worth noticing that $e_{\mu \nu}$ and $d_{\mu \nu}^{-}$is not linearly independent of $f_{\mu \nu}^{+}$and $f_{\mu \nu}^{-}$, respectively. From this, one finds that $\mathcal{O}_{5}^{-}-2 \mathcal{O}_{4}^{-}=4 \operatorname{Tr}\left(f_{\mu \nu}^{-} e^{\mu \nu}\right) \neq \mathcal{O}_{3}$.

\section{APPENDIX C: EW CHIRAL LAGRANGIAN AND OPERATORS}

\section{Building blocks of low-energy EW chiral Lagrangian}

The (pseudo-)scalar $\mathbf{T}$ and vector chiral fields $\mathbf{V}_{\mu}$ are defined as $[34,49,50]$

$$
\mathbf{T} \equiv \mathbf{U} \sigma^{3} \mathbf{U}^{\dagger}, \quad \mathbf{V}_{\mu} \equiv\left(\mathbf{D}_{\mu} \mathbf{U}\right) \mathbf{U}^{\dagger},
$$

where $\mathbf{U}$ is the three Goldstone bosons (GBs) in the coset $S U(2)_{L} \times S U(2)_{R} / S U(2)_{C}$ and are parametrized as the longitudinal components of the SM gauge bosons by a nonlinear $\sigma$ model as a dimensionless unitary matrix $\mathbf{U}$ at low energies as

$$
\mathbf{U}=\exp \left(i \frac{1}{v} \sigma^{a} \varphi^{a}\right),
$$

where $v$ is the scale associated with the SM GBs, and $\sigma^{a}$ are the usual Pauli matrices. The dimensionless unitary SM GB matrix transforms as a bi-doublet under the global $S U(2)_{L} \times S U(2)_{R}$ symmetry as

$$
\mathbf{U} \rightarrow \mathfrak{g}_{L} \mathbf{U} \mathfrak{g}_{R}^{\dagger} .
$$

After EWSB, the global $S U(2)_{L} \times S U(2)_{R}$ symmetry is spontaneously broken down to the diagonal $S U(2)_{C}$ in terms of custodial symmetry, and explicitly broken by gauging the $U(1)_{Y}$ hypercharge and by the fermion mass splittings.
The covariant derivatives are ${ }^{6}$

$$
\mathbf{D}_{\mu} \mathbf{U} \equiv \partial_{\mu} \mathbf{U}+i g \mathbf{W}_{\mu} \mathbf{U}-i g^{\prime} B_{\mu} \mathbf{U} \sigma_{3} / 2,
$$

where $\mathbf{W}_{\mu} \equiv W_{\mu}^{a} \sigma_{a} / 2$ with $W_{\mu}^{a}$ and $B_{\mu}$ denote the $S U(2)_{L}$ and $U(1)_{Y}$ gauge bosons, respectively, and $g, g^{\prime}$ are the corresponding gauge coupling. Both $\mathbf{T}$ and $\mathbf{V}_{\mu}$ transform in the adjoint symmetry representation of $S U(2)_{L}$ as

$$
\mathbf{T} \rightarrow \mathfrak{g}_{L} \mathbf{T} \mathfrak{g}_{L}^{\dagger}, \quad \mathbf{V}_{\mu} \rightarrow \mathfrak{g}_{L} \mathbf{V}_{\mu} \mathfrak{g}_{L}^{\dagger},
$$

while the chiral scalar field $\mathbf{T}$ breaks explicitly the $S U(2)_{R}$ symmetry and is not invariant under $S U(2)_{C}$. Thus, it can be considered as a custodial symmetry breaking term. Thus, the covariant derivative $\mathbf{D}_{\mu}$ denotes that in the adjoint representation of $\mathrm{SU}(2)_{L}$, i.e., when acting upon $\mathbf{V}_{\mu}$, is given by

$$
\mathbf{D}_{\mu} \mathbf{V}_{\nu} \equiv \partial_{\mu} \mathbf{V}_{\nu}+i g\left[\mathbf{W}_{\mu}, \mathbf{V}_{\nu}\right]
$$

and satisfies a useful identity $\left(\mathbf{D}_{\mu} \mathbf{U}\right)^{\dagger}=-\mathbf{U}^{-1}\left(\mathbf{D}_{\mu} \mathbf{U}\right) \mathbf{U}^{\dagger}$. In this case, one obtains frequently useful equalities as [34]

$$
\begin{aligned}
\mathbf{V}_{\mu \nu} & \equiv \mathbf{D}_{\mu} \mathbf{V}_{\nu}-\mathbf{D}_{\nu} \mathbf{V}_{\mu}=i g \mathbf{W}_{\mu \nu}-i \frac{g^{\prime}}{2} B_{\mu \nu} \mathbf{T}+\left[\mathbf{V}_{\mu}, \mathbf{V}_{\nu}\right], \\
\mathbf{D}_{\mu} \mathbf{T} & =\left[\mathbf{V}_{\mu}, \mathbf{T}\right],\left[\mathbf{D}_{\mu}, \mathbf{D}_{\nu}\right] \mathcal{O}=i g\left[\mathbf{W}_{\mu \nu}, \mathcal{O}\right],
\end{aligned}
$$

where $\mathcal{O}$ is a generic operator covariant under $S U(2)_{L}$ and invariant under $U(1)_{Y}$.

It is worth noticing that it is concise to use the building block $\mathbf{V}_{\mu}$, comparing to use the explicit expression of $\mathbf{U}$ as

$$
\operatorname{Tr}\left(\mathbf{V}_{\mu} \mathbf{V}^{\mu}\right)=\operatorname{Tr}\left[\mathbf{U}^{\dagger}\left(\mathbf{D}_{\mu} \mathbf{U}\right) \mathbf{U}^{\dagger}\left(\mathbf{D}_{\mu} \mathbf{U}\right)\right],
$$

where in the last equality, we have used the Hermitian condition for the Lagrangian up to kinetic terms.

\section{Low-energy EW chiral Lagrangian}

\section{a. Higgs singlet}

The physical Higgs $h$ is an iso-singlet of the SM gauge symmetry with vacuum expectation value at EW scale $v \approx 246 \mathrm{GeV}$. In the low-energy effective Lagrangian, there are four pure Higgs operators. One is that with two derivatives, and the other three are those with four derivatives as [47]

$$
\begin{aligned}
\mathcal{L}_{H} & =\frac{1}{2}\left(\partial_{\mu} h\right)\left(\partial^{\mu} h\right) \\
\mathcal{L}_{\square H} & =\frac{1}{v^{2}}(\square h)^{2} \\
\mathcal{L}_{\Delta H} & =\frac{1}{v^{3}}\left(\partial_{\mu} h\right)\left(\partial^{\mu} h\right) \square h \\
\mathcal{L}_{D H} & =\frac{1}{v^{4}}\left[\left(\partial_{\mu} h\right)\left(\partial^{\mu} h\right)\right]^{2},
\end{aligned}
$$

where $\square=\partial_{\mu} \partial^{\mu}$.

\footnotetext{
${ }^{6}$ The bold of the characters implies that we have adopt abbreviations for $S U(2)_{L}$ generators.
} 


\section{b. $C P$-even case}

Operators with two derivatives are [35]

$$
\begin{aligned}
& \mathcal{L}_{C}=-\frac{v^{2}}{4} \operatorname{Tr}\left(\mathbf{V}^{\mu} \mathbf{V}_{\mu}\right), \\
& \mathcal{L}_{T}=\frac{v^{2}}{4} \operatorname{Tr}\left(\mathbf{T} \mathbf{V}_{\mu}\right) \operatorname{Tr}\left(\mathbf{T} \mathbf{V}^{\mu}\right),
\end{aligned}
$$

where $C$ and $T$ indicates the custodial preserving and custodial breaking, respectively.

For operators with four derivatives one has ${ }^{7}[34,36,38$, $45,45,47,49,53]$

$$
\begin{aligned}
\mathcal{L}_{B} & =-\frac{g^{\prime 2}}{4} B_{\mu \nu} B^{\mu \nu}, \\
\mathcal{L}_{W} & =-\frac{g^{2}}{2} \operatorname{Tr}\left(\mathbf{W}_{\mu \nu} \mathbf{W}^{\mu \nu}\right), \\
\mathcal{L}_{1} & =g g^{\prime} B_{\mu \nu} \operatorname{Tr}\left(\mathbf{T} \mathbf{W}^{\mu \nu}\right), \\
\mathcal{L}_{2} & =i g^{\prime} B_{\mu \nu} \operatorname{Tr}\left(\mathbf{T}\left[\mathbf{V}^{\mu}, \mathbf{V}^{\nu}\right]\right), \\
\mathcal{L}_{3} & =i g \operatorname{Tr}\left(\mathbf{W}_{\mu \nu}\left[\mathbf{V}^{\mu}, \mathbf{V}^{\nu}\right]\right), \\
\mathcal{L}_{4} & =i g^{\prime} B_{\mu \nu} \operatorname{Tr}\left(\mathbf{T} \mathbf{V}^{\mu}\right) \partial^{\nu}(h / v), \\
\mathcal{L}_{5} & =i g \operatorname{Tr}\left(\mathbf{W}_{\mu \nu} \mathbf{V}^{\mu}\right) \partial^{\nu}(h / v), \\
\mathcal{L}_{6} & =\left[\operatorname{Tr}\left(\mathbf{V}_{\mu} \mathbf{V}^{\mu}\right)\right]^{2}, \\
\mathcal{L}_{7} & =\operatorname{Tr}\left(\mathbf{V}_{\mu} \mathbf{V}^{\mu}\right) \partial_{\nu} \partial^{\nu}(h / v), \\
\mathcal{L}_{8} & =\operatorname{Tr}\left(\mathbf{V}_{\mu} \mathbf{V}_{\nu}\right) \partial^{\mu}(h / v) \partial^{\nu}(h / v), \\
\mathcal{L}_{9} & =\operatorname{Tr}\left[\left(\mathbf{D}_{\mu} \mathbf{V}^{\mu}\right)^{2}\right], \\
\mathcal{L}_{10} & =\operatorname{Tr}\left(\mathbf{V}_{\nu} \mathbf{D}_{\mu} \mathbf{V}^{\mu}\right) \partial^{\nu}(h / v), \\
\mathcal{L}_{11} & =\left[\operatorname{Tr}\left(\mathbf{V}_{\mu} \mathbf{V}_{\nu}\right)\right]^{2}, \\
\mathcal{L}_{12} & =g^{2}\left[\operatorname{Tr}\left(\mathbf{T} \mathbf{W}_{\mu \nu}\right)\right]^{2}, \\
\mathcal{L}_{13} & =i g \operatorname{Tr}\left(\mathbf{T} \mathbf{W}_{\mu \nu}\right) \operatorname{Tr}\left(\mathbf{T}\left[\mathbf{V}^{\mu}, \mathbf{V}^{\nu}\right]\right), \\
\mathcal{L}_{14} & =g \epsilon_{\mu \nu \rho \lambda} \operatorname{Tr}\left(\mathbf{T} \mathbf{V}^{\mu}\right) \operatorname{Tr}\left(\mathbf{V}^{\nu} \mathbf{W}^{\rho \lambda}\right), \\
\mathcal{L}_{15} & =\operatorname{Tr}\left(\mathbf{T} \mathbf{D}_{\mu} \mathbf{V}^{\mu}\right) \operatorname{Tr}\left(\mathbf{T} \mathbf{D}_{\nu} \mathbf{V}^{\nu}\right), \\
\mathcal{L}_{16} & =\operatorname{Tr}\left(\left[\mathbf{T}, \mathbf{V}_{\nu}\right] \mathbf{D}_{\mu} \mathbf{V}^{\mu}\right) \operatorname{Tr}\left(\mathbf{T} \mathbf{V}^{\nu}\right), \\
\mathcal{L}_{17} & =i g \operatorname{Tr}\left(\mathbf{T} \mathbf{W}_{\mu \nu}\right) \operatorname{Tr}\left(\mathbf{T} \mathbf{V}^{\mu}\right) \partial^{\nu}(h / v), \\
\mathcal{L}_{18} & =\operatorname{Tr}\left(\mathbf{T}\left[\mathbf{V}_{\mu}, \mathbf{V}_{\nu}\right]\right) \operatorname{Tr}\left(\mathbf{T} \mathbf{V}^{\mu}\right) \partial^{\nu}(h / v),
\end{aligned}
$$

\footnotetext{
${ }^{7}$ These $26 p^{4}$ operators in the EW chiral Lagrangian are redundant if the fermion sector is included [46]. Thus, we only need to focus on the NMCHM setup with only composite vector boson states included.
}

$$
\begin{aligned}
\mathcal{L}_{19} & =\operatorname{Tr}\left(\mathbf{T} \mathbf{D}_{\mu} \mathbf{V}^{\mu}\right) \operatorname{Tr}\left(\mathbf{T} \mathbf{V}_{\nu}\right) \partial^{\nu}(h / v), \\
\mathcal{L}_{20} & =\operatorname{Tr}\left(\mathbf{V}_{\mu} \mathbf{V}^{\mu}\right) \partial_{\nu}(h / v) \partial^{\nu}(h / v), \\
\mathcal{L}_{21} & =\left[\operatorname{Tr}\left(\mathbf{T} \mathbf{V}_{\mu}\right)\right]^{2} \partial_{\nu}(h / v) \partial^{\nu}(h / v), \\
\mathcal{L}_{22} & =\operatorname{Tr}\left(\mathbf{T} \mathbf{V}_{\mu}\right) \operatorname{Tr}\left(\mathbf{T} \mathbf{V}_{\nu}\right) \partial^{\mu}(h / v) \partial^{\nu}(h / v), \\
\mathcal{L}_{23} & =\operatorname{Tr}\left(\mathbf{V}_{\mu} \mathbf{V}^{\mu}\right)\left[\operatorname{Tr}\left(\mathbf{T} \mathbf{V}^{\nu}\right)\right]^{2}, \\
\mathcal{L}_{24} & =\operatorname{Tr}\left(\mathbf{V}_{\mu} \mathbf{V}_{\nu}\right) \operatorname{Tr}\left(\mathbf{T} \mathbf{V}^{\mu}\right) \operatorname{Tr}\left(\mathbf{T} \mathbf{V}^{\nu}\right), \\
\mathcal{L}_{25} & =\left[\operatorname{Tr}\left(\mathbf{T} \mathbf{V}_{\mu}\right)\right]\left[\operatorname{Tr}\left(\mathbf{T} \mathbf{V}^{\mu}\right)\right] \partial_{\nu} \partial^{\nu}(h / v), \\
\mathcal{L}_{26} & =\left[\operatorname{Tr}\left(\mathbf{T} \mathbf{V}_{\mu}\right) \operatorname{Tr}\left(\mathbf{T} \mathbf{V}_{\nu}\right)\right]^{2},
\end{aligned}
$$

where the first 13 Lagrangians $\mathcal{L}_{B, W, 1, \ldots, 13}$ correspond to the custodial preserving ones, while the residue corresponds to the custodial breaking ones, which describes treelevel effects of custodial breaking sources beyond the SM ones. Since the gauging of the SM symmetry breaks explicitly the custodial symmetries, these custodial symmetry breaking operators are generated due to the quantum corrections induced by the SM interactions. The covariant derivatives of $\mathbf{V}_{\mu}$ are defined as [34]

$$
\mathbf{D}_{\mu} \mathbf{V}_{\nu} \equiv \partial_{\mu} \mathbf{V}_{\nu}+i g\left[W_{\mu}, \mathbf{V}_{\nu}\right]
$$

In the absence of a light $C P$-even Higgs-like scalar singlet $h$ in the low-energy spectrum, the 12 operators containing derivatives of Higgs are absent. Thus, there are a complete 18 (independent) $\mathrm{CP}$-even operators: those preserving $S U(2)_{L} \times U(1)_{Y}$ symmetry.

Among the operators, two $S U(2)_{C}$ custodial symmetry preserving and three custodial violating operators $\mathcal{L}_{6,11 ; 23,24,26}$ exhibit quartic vector-boson interactions, which leads to new anomalous quartic couplings [74] such as $Z_{\mu} Z_{\nu} Z^{\mu} Z^{\nu}, W_{\mu}^{+} W_{\nu}^{-} Z^{\mu} Z^{\nu}$, and $W_{\mu}^{+} W_{\nu}^{-} W^{+\mu} W^{-\nu}$.

The $C P$-even low-energy effective Lagrangian in Eq. (C11) can be expressed more explicitly in the unitary gauge as

$$
\begin{aligned}
\mathcal{L}_{C}= & \frac{1}{8} v^{2}\left[g^{2}\left[\left(W_{\mu}^{1}\right)^{2}+\left(W_{\mu}^{2}\right)^{2}\right]+\left(g W_{\mu}^{3}-g^{\prime} B_{\mu}\right)^{2}\right], \\
\mathcal{L}_{T}= & -\frac{1}{4} v^{2}\left(g W_{\mu}^{3}-g^{\prime} B_{\mu}\right)^{2}, \\
\mathcal{L}_{B}= & -\frac{g^{\prime 2}}{4} B_{\mu \nu} B^{\mu \nu}, \\
\mathcal{L}_{W}= & -\frac{g^{2}}{4} W_{\mu \nu}^{a} W^{a \mu \nu}, \\
\mathcal{L}_{1}= & g g^{\prime} B_{\mu \nu} W^{3 \mu \nu}, \\
\mathcal{L}_{2}= & g^{2} g^{\prime} B^{\mu \nu}\left(W_{\mu}^{1} W_{\nu}^{2}-W_{\mu}^{2} W_{\nu}^{1}\right), \\
\mathcal{L}_{4}= & g^{\prime} B^{\mu \nu}\left(g^{\prime} B_{\mu}-g W_{\mu}^{3}\right) \partial_{\nu} h / v, \\
\mathcal{L}_{3}= & \frac{g^{2}}{2}\left[g W^{3 \mu \nu}\left(W_{\mu}^{1} W_{\nu}^{2}-W_{\mu}^{2} W_{\nu}^{1}\right)\right. \\
& +\left(g W_{\nu}^{3}-g^{\prime} B_{\nu}\right)\left(W^{1 \mu \nu} W_{\mu}^{2}-W_{\mu}^{1} W^{2 \mu \nu}\right) \\
& \left.-\left(g W_{\mu}^{3}-g^{\prime} B_{\mu}\right)\left(W^{1 \mu \nu} W_{\nu}^{2}-W_{\nu}^{1} W^{2 \mu \nu}\right)\right],
\end{aligned}
$$




$$
\begin{aligned}
& \mathcal{L}_{5}=-\frac{g}{2}\left[g\left(W_{\mu}^{1} W^{1 \mu \nu}+W_{\mu}^{2} W^{2 \mu \nu}\right)\right. \\
& \left.+\left(g W_{\mu}^{3}-g^{\prime} B_{\mu}\right) W^{3 \mu \nu}\right] \partial_{\nu} h / v, \\
& \mathcal{L}_{6}=\frac{1}{4}\left[g^{2}\left(W_{\mu}^{1}\right)^{2}+g^{2}\left(W_{\mu}^{2}\right)^{2}+\left(g W_{\mu}^{3}-g^{\prime} B_{\mu}\right)^{2}\right]^{2}, \\
& \mathcal{L}_{7}=-\frac{1}{2 v}\left[g^{2}\left(\left(W_{\mu}^{1}\right)^{2}+\left(W_{\mu}^{2}\right)^{2}\right)+\left(g W_{\mu}^{3}-g^{\prime} B_{\mu}\right)^{2}\right] \square h, \\
& \mathcal{L}_{8}=-\frac{1}{2 v^{2}}\left[g^{2}\left(W_{\mu}^{1} W_{\nu}^{1}+W_{\mu}^{2} W_{\nu}^{2}\right)\right. \\
& \left.+\left(g W_{\mu}^{3}-g^{\prime} B_{\mu}\right)\left(g W_{\nu}^{3}-g^{\prime} B_{\nu}\right)\right] \partial^{\mu} h \partial^{\nu} h, \\
& \mathcal{L}_{9}=-\frac{1}{2}\left[g^{2}\left(\partial_{\mu} W^{1 \mu}+g^{\prime} B_{\mu} W^{2 \mu}\right)^{2}\right. \\
& +g^{2}\left(\partial_{\mu} W^{2 \mu}-g^{\prime} B_{\mu} W^{1 \mu}\right)^{2} \\
& \left.+\left(g \partial_{\mu} W^{3 \mu}-g^{\prime} \partial_{\mu} B^{\mu}\right)^{2}\right] \\
& \mathcal{L}_{10}=-\frac{1}{2 v}\left[g^{2}\left(W_{\mu}^{1} \partial_{\nu} W^{1 \nu}+W_{\mu}^{2} \partial_{\nu} W^{2 \nu}\right)\right. \\
& \left.+\left(g W_{\mu}^{3}-g^{\prime} B_{\mu}\right) \partial^{\nu}\left(g W_{\nu}^{3}-g^{\prime} B_{\nu}^{3}\right)\right] \partial^{\mu} h, \\
& \mathcal{L}_{11}=\frac{1}{4}\left[g^{2}\left(W_{\mu}^{1} W_{\nu}^{1}+W_{\mu}^{2} W_{\nu}^{2}\right)\right. \\
& \left.+\left(g W_{\mu}^{3}-g^{\prime} B_{\mu}\right)\left(g W_{\nu}^{3}-g^{\prime} B_{\nu}\right)\right]^{2}, \\
& \mathcal{L}_{12}=g^{2} W^{3 \mu \nu} W_{\mu \nu}^{3}, \\
& \mathcal{L}_{13}=g^{3} W^{3 \mu \nu}\left(W_{\mu}^{1} W_{\nu}^{2}-W_{\nu}^{1} W_{\mu}^{2}\right), \\
& \mathcal{L}_{14}=g\left(g W^{3 \mu}-g^{\prime} B^{\mu}\right)\left[g\left(W^{1 \nu} \tilde{W}_{\mu \nu}^{1}+W^{2 \nu} \tilde{W}_{\mu \nu}^{2}\right)\right. \\
& \left.+\left(g W^{3 \nu}-g^{\prime} B^{\nu}\right) \tilde{W}_{\mu \nu}^{3}\right] \\
& \mathcal{L}_{15}=-\left(g \partial_{\mu} W^{3 \mu}-g^{\prime} \partial_{\mu} B^{\mu}\right)^{2}, \\
& \mathcal{L}_{16}=-g^{2}\left(g W^{3 \nu}-g^{\prime} B^{\nu}\right)\left[\left(W_{\nu}^{2} \partial_{\mu} W^{1 \mu}-W_{\nu}^{1} \partial_{\mu} W^{2 \mu}\right)\right. \\
& \left.+g^{\prime} B^{\mu}\left(W_{\mu}^{1} W_{\nu}^{1}+W_{\mu}^{2} W_{\nu}^{2}\right)\right] \\
& \mathcal{L}_{17}=-\frac{1}{v} g\left(g W_{\mu}^{3}-g^{\prime} B_{\mu}\right) W^{3 \mu \nu} \partial_{\nu} h, \\
& \mathcal{L}_{18}=\frac{1}{v} g^{2}\left(g W^{3 \mu}-g^{\prime} B^{\mu}\right)\left(W_{\mu}^{1} W_{\nu}^{2}-W_{\mu}^{2} W_{\nu}^{1}\right) \partial^{\nu} h, \\
& \mathcal{L}_{19}=-\frac{1}{v}\left(g W^{3 \nu}-g^{\prime} B^{\nu}\right)\left(g \partial_{\mu} W^{3 \mu}-g^{\prime} \partial_{\mu} B^{\mu}\right) \partial_{\nu} h, \\
& \mathcal{L}_{20}=-\frac{1}{2 v^{2}}\left[g^{2}\left(\left(W_{\mu}^{1}\right)^{2}+\left(W_{\mu}^{2}\right)^{2}\right)+\left(g W_{\mu}^{3}-g^{\prime} B_{\mu}\right)^{2}\right]\left(\partial_{\nu} h\right)^{2}, \\
& \mathcal{L}_{21}=-\frac{1}{v^{2}}\left(g W^{3 \mu}-g^{\prime} B^{\mu}\right)^{2} \partial_{\nu} h \partial^{\nu} h, \\
& \mathcal{L}_{22}=-\frac{1}{v^{2}}\left(g W^{3 \mu}-g^{\prime} B^{\mu}\right)\left(g W^{3 \nu}-g^{\prime} B^{\nu}\right) \partial_{\mu} h \partial_{\nu} h, \\
& \mathcal{L}_{23}=\frac{1}{2}\left(g W^{3 \nu}-g^{\prime} B^{\nu}\right)^{2}\left[g^{2}\left(W^{1 \mu} W_{\mu}^{1}+W^{2 \nu} W_{\nu}^{2}\right)\right. \\
& \left.+\left(g W^{3 \mu}-g^{\prime} B^{\mu}\right)^{2}\right], \\
& \mathcal{L}_{24}=\frac{1}{2}\left(g W^{3 \mu}-g^{\prime} B^{\mu}\right)\left(g W^{3 \nu}-g^{\prime} B^{\nu}\right) \times \\
& {\left[g^{2}\left(W_{\mu}^{1} W_{\nu}^{1}+W_{\mu}^{2} W_{\nu}^{2}\right)+\left(g W_{\mu}^{3}-g^{\prime} B_{\mu}\right)\left(g W_{\nu}^{3}-g^{\prime} B_{\nu}\right)\right],}
\end{aligned}
$$

$$
\begin{aligned}
& \mathcal{L}_{25}=-\frac{1}{v}\left(g W^{3 \mu}-g^{\prime} B^{\mu}\right)^{2} \square h, \\
& \mathcal{L}_{26}=\left(g W^{3 \mu}-g^{\prime} B^{\mu}\right)^{2}\left(g W^{3 \nu}-g^{\prime} B^{\nu}\right)^{2},
\end{aligned}
$$

where $\tilde{W}^{a \mu \nu}=\epsilon^{\mu \nu \rho \sigma} W_{\rho \sigma}^{a} / 2$. Note that in the weak coupling limit, i.e., $f \rightarrow \infty$ or $\xi \rightarrow 0, \mathcal{L}_{4}$ just recovers that in the low-energy effective Lagrangian, while $\mathcal{L}_{4}^{(s)}$ just decoupled. It is worth noticing that for NMCHM with symmetry breaking pattern as $S O(6) / S O(5)$, the custodial violating operator $\mathcal{L}_{T}=-2 \mathcal{L}_{C}+\cdots$ is not independent of $\mathcal{L}_{C}$.

\section{c. $C P$-odd case}

For operators with two derivatives one has

$$
\begin{aligned}
& \mathcal{L}_{\tilde{C}}=0, \\
& \mathcal{L}_{\tilde{T}}=i \frac{v^{2}}{4} \operatorname{Tr}\left(\mathbf{T D}^{\mu} \mathbf{V}_{\mu}\right),
\end{aligned}
$$

where $\tilde{C}$ and $\tilde{T}$ indicates the custodial preserving and custodial breaking, respectively.

For operators with four derivatives one has [48,52,53]

$$
\begin{aligned}
& \mathcal{L}_{\tilde{B}}=-\frac{g^{\prime 2}}{4} \tilde{B}_{\mu \nu} B^{\mu \nu}, \\
& \mathcal{L}_{\tilde{W}}=-\frac{g^{2}}{2} \operatorname{Tr}\left(\tilde{\mathbf{W}}_{\mu \nu} \mathbf{W}^{\mu \nu}\right), \\
& \mathcal{L}_{\tilde{1}}=2 g g^{\prime} B_{\mu \nu} \operatorname{Tr}\left(\mathbf{T} \tilde{\mathbf{W}}^{\mu \nu}\right), \\
& \mathcal{L}_{\tilde{2}}=2 i g^{\prime} \tilde{B}_{\mu \nu} \operatorname{Tr}\left(\mathbf{T} \mathbf{V}^{\mu}\right) \partial^{\nu}(h / v), \\
& \mathcal{L}_{\tilde{3}}=2 i g \operatorname{Tr}\left(\tilde{\mathbf{W}}^{\mu \nu} \mathbf{V}_{\mu}\right) \partial_{\nu}(h / v), \\
& \mathcal{L}_{\tilde{4}}=g \operatorname{Tr}\left(\mathbf{W}^{\mu \nu} \mathbf{V}_{\mu}\right) \operatorname{Tr}\left(\mathbf{T} \mathbf{V}_{\nu}\right), \\
& \mathcal{L}_{\tilde{5}}=i \operatorname{Tr}\left(\mathbf{V}^{\mu} \mathbf{V}^{\nu}\right) \operatorname{Tr}\left(\mathbf{T} \mathbf{V}_{\mu}\right) \partial_{\nu}(h / v), \\
& \mathcal{L}_{\tilde{6}}=i \operatorname{Tr}\left(\mathbf{V}^{\mu} \mathbf{V}_{\mu}\right) \operatorname{Tr}\left(\mathbf{T} \mathbf{V}^{\nu}\right) \partial_{\nu}(h / v), \\
& \mathcal{L}_{\tilde{7}}=g \operatorname{Tr}\left(\mathbf{T}\left[\mathbf{W}^{\mu \nu}, \mathbf{V}_{\mu}\right]\right) \partial_{\nu}(h / v), \\
& \mathcal{L}_{\tilde{8}}=2 g^{2} \operatorname{Tr}\left(\mathbf{T} \tilde{\mathbf{W}}^{\mu \nu}\right) \operatorname{Tr}\left(\mathbf{T} \mathbf{W}_{\mu \nu}\right), \\
& \mathcal{L}_{\tilde{g}}=2 i g \operatorname{Tr}\left(\mathbf{T} \tilde{\mathbf{W}}^{\mu \nu}\right) \operatorname{Tr}\left(\mathbf{T} \mathbf{V}_{\mu}\right) \partial_{\nu}(h / v), \\
& \mathcal{L}_{10}=i \operatorname{Tr}\left(\mathbf{V}^{\mu} \mathbf{D}^{\nu} \mathbf{V}_{\nu}\right) \operatorname{Tr}\left(\mathbf{T} \mathbf{V}_{\mu}\right), \\
& \mathcal{L}_{\tilde{1} 1}=i \operatorname{Tr}\left(\mathbf{T D}^{\mu} \mathbf{V}_{\mu}\right) \operatorname{Tr}\left(\mathbf{V}^{\nu} \mathbf{V}_{\nu}\right), \\
& \mathcal{L}_{\tilde{12}}=i \operatorname{Tr}\left(\left[\mathbf{V}^{\mu}, \mathbf{T}\right] \mathbf{D}^{\nu} \mathbf{V}_{\nu}\right) \partial_{\mu}(h / v), \\
& \mathcal{L}_{\tilde{13}}=i \operatorname{Tr}\left(\mathbf{T D}^{\mu} \mathbf{V}_{\mu}\right) \partial^{\nu} \partial_{\nu}(h / v), \\
& \mathcal{L}_{\tilde{1} 4}=i \operatorname{Tr}\left(\mathbf{T D}^{\mu} \mathbf{V}_{\mu}\right) \partial^{\nu}(h / v) \partial_{\nu}(h / v), \\
& \mathcal{L}_{15}=i \operatorname{Tr}\left(\mathbf{T V}^{\mu}\right)\left(\operatorname{Tr}\left(\mathbf{T V}^{\nu}\right)\right)^{2} \partial_{\mu}(h / v), \\
& \mathcal{L}_{\tilde{1}}=i \operatorname{Tr}\left(\mathbf{T D}^{\mu} \mathbf{V}_{\mu}\right)\left(\operatorname{Tr}\left(\mathbf{T V}^{\nu}\right)\right)^{2},
\end{aligned}
$$

where the first five Lagrangians $\mathcal{L}_{\tilde{B}, \tilde{W}, \tilde{1}, \tilde{2}, \tilde{3}}$ correspond to the custodial preserving ones, while the residue operators 
correspond to (tree-level) custodial breaking ones. In the custodial breaking class, the presence of the scalar chiral field $\mathbf{T}$ implies that the custodial symmetry is violating. The dual tensors are defined by $\tilde{B}_{\mu \nu} \equiv \epsilon_{\mu \nu \rho \sigma} B^{\rho \sigma}$ and $\tilde{\mathbf{W}}_{\mu \nu} \equiv \epsilon_{\mu \nu \rho \sigma} \mathbf{W}^{\rho \sigma}$. The covariant derivative of $\mathbf{V}$ is defined in Eq. (C6). In the absence of a light Higgs-like, i.e., $C P$-odd scalar singlet $h$ in the low-energy spectrum, the 10 operators containing derivatives of Higgs are absent. Thus, there are a complete nine (independent) $C P$-even operators, those preserving $S U(2)_{L} \times U(1)_{Y}$ symmetry.

The $C P$-odd low-energy effective Lagrangian in Eq. (C15) can be expressed more explicitly in the unitary gauge as

$$
\begin{aligned}
\mathcal{L}_{\tilde{C}}= & 0, \\
\mathcal{L}_{\tilde{T}}= & -\frac{i}{4} v^{2}\left[\partial_{\mu}\left(g W^{3 \mu}-g^{\prime} B^{\mu}\right)\right]^{2}, \\
\mathcal{L}_{\tilde{W}}= & -\frac{1}{4} g^{\prime 2} \tilde{W}_{\mu \nu}^{a} W^{a \mu \nu}, \\
\mathcal{L}_{\tilde{B}}= & -\frac{1}{4} g^{\prime 2} \tilde{B}_{\mu \nu} B^{\mu \nu}, \\
\mathcal{L}_{\tilde{1}}= & 2 g g^{\prime} B_{\mu \nu} \tilde{W}^{3 \mu \nu}, \\
\mathcal{L}_{\tilde{2}}= & -\frac{2}{v} g^{\prime} \tilde{B}^{\mu \nu}\left(g W_{\mu}^{3}-g^{\prime} B_{\mu}\right) \partial_{\nu} h, \\
\mathcal{L}_{\tilde{3}}= & -\frac{1}{v}\left[g^{2}\left(W_{\mu}^{1} \tilde{W}^{1 \mu \nu}+W_{\mu}^{2} \tilde{W}^{2 \mu \nu}\right)\right. \\
& \left.+g \tilde{W}^{3 \mu \nu}\left(g W_{\mu}^{3}-g^{\prime} B_{\mu}\right)\right] \partial_{\nu} h, \\
\mathcal{L}_{\tilde{4}}= & -\frac{1}{2}\left[g^{2}\left(W_{\mu}^{1} W^{1 \mu \nu}+W_{\mu}^{2} W^{2 \mu \nu}\right)\right. \\
& \left.\left.+g\left(g W_{\mu}^{3}-g^{\prime} B_{\mu}\right) W^{3 \mu \nu}\right)\right]\left(g W_{\nu}^{3}-g^{\prime} B_{\nu}\right),
\end{aligned}
$$

$$
\begin{aligned}
& \mathcal{L}_{\tilde{5}}=\frac{1}{2 v}\left(g W_{\mu}^{3}-g^{\prime} B_{\mu}\right)\left[g^{2}\left(W^{1 \mu} W^{1 \nu}+W^{2 \mu} W^{2 \nu}\right)\right. \\
& \left.+\left(g W^{3 \mu}-g^{\prime} B^{\mu}\right)\left(g W^{3 \nu}-g^{\prime} B^{\nu}\right)\right] \partial_{\nu} h, \\
& \mathcal{L}_{\tilde{6}}=\frac{1}{2 v}\left(g W^{3 \nu}-g^{\prime} B^{\nu}\right)\left[\left(g W_{\mu}^{1}+g W_{\mu}^{2}\right)^{2}\right. \\
& \left.+\left(g W_{\mu}^{3}-g^{\prime} B_{\mu}\right)^{2}\right] \partial_{\nu} h, \\
& \mathcal{L}_{\tilde{7}}=\frac{1}{v} g^{2}\left(W_{\mu}^{1} W^{2 \mu \nu}-W_{\mu}^{2} W^{1 \mu \nu}\right) \partial_{\nu} h, \\
& \mathcal{L}_{\tilde{8}}=2 g^{2} W_{\mu \nu}^{3} \tilde{W}^{3 \mu \nu}, \\
& \mathcal{L}_{\tilde{9}}=-\frac{2 g}{v}\left(g W_{\mu}^{3}-g^{\prime} B_{\mu}\right) \tilde{W}^{3 \mu \nu} \partial_{\nu} h, \\
& \mathcal{L}_{\tilde{10}}=\frac{1}{2}\left(g W_{\mu}^{3}-g^{\prime} B_{\mu}\right)\left[g^{2}\left(W^{1 \mu} \partial_{\nu} W^{1 \nu}+W^{2 \mu} \partial_{\nu} W^{2 \nu}\right)\right. \\
& +\left(g W^{3 \mu}-g^{\prime} B^{\mu}\right)\left(g \partial_{\nu} W^{3 \nu}-g^{\prime} \partial_{\nu} B^{\nu}\right) \\
& \left.+g^{2} g^{\prime} B^{\nu}\left(W^{1 \mu} W_{\nu}^{2}-W^{2 \mu} W_{\nu}^{1}\right)\right] \text {, } \\
& \mathcal{L}_{\tilde{11}}=\frac{1}{2}\left[g^{2}\left(W_{\nu}^{1} W^{1 \nu}+W_{\nu}^{2} W^{2 \nu}\right)+\left(g W_{\nu}^{3}-g^{\prime} B_{\nu}\right)^{2}\right] \\
& \times\left(g \partial_{\mu} W^{3 \mu}-g^{\prime} \partial_{\mu} B^{\mu}\right), \\
& \mathcal{L}_{\tilde{12}}=\frac{g^{2}}{v}\left[g^{\prime} B_{\nu}\left(W^{1 \mu} W^{1 \nu}+W^{2 \mu} W^{2 \nu}\right)\right. \\
& \left.+\left(W^{2 \mu} \partial_{\nu} W^{1 \nu}-W^{1 \mu} \partial_{\nu} W^{2 \nu}\right)\right] \partial_{\mu} h, \\
& \mathcal{L}_{\tilde{13}}=-\frac{1}{v}\left(g \partial_{\mu} W^{3 \mu}-g^{\prime} \partial_{\mu} B^{\mu}\right) \square h, \\
& \mathcal{L}_{\tilde{14}}=-\frac{1}{v^{2}}\left(g \partial_{\mu} W^{3 \mu}-g^{\prime} \partial_{\mu} B^{\mu}\right)\left(\partial_{\nu} h\right)^{2}, \\
& \mathcal{L}_{15}=\frac{1}{v}\left(g W^{3 \nu}-g^{\prime} B^{\nu}\right)^{2}\left(g W^{3 \mu}-g^{\prime} B^{\mu}\right) \partial_{\mu} h, \\
& \mathcal{L}_{\tilde{16}}=\left(g W^{3 \nu}-g^{\prime} B^{\nu}\right)^{2}\left(g \partial_{\mu} W^{3 \mu}-g^{\prime} \partial_{\mu} B^{\mu}\right) .
\end{aligned}
$$

[1] S. Weinberg, A Model of Leptons, Phys. Rev. Lett. 19, 1264 (1967).

[2] C.-N. Yang and R. L. Mills, Conservation of isotopic spin and isotopic gauge invariance, Phys. Rev. 96, 191 (1954).

[3] E. S. Abers and B. W. Lee, Gauge theories, Phys. Rep. 9, 1 (1973).

[4] S. Weinberg, The Quantum Theory of Fields. Vol. 2: Modern Applications (Cambridge University Press, Cambridge, England, 2013).

[5] D. B. Kaplan and H. Georgi, SU(2) $\times \mathrm{U}(1)$ breaking by vacuum misalignment, Phys. Lett. 136B, 183 (1984).

[6] D. B. Kaplan, H. Georgi, and S. Dimopoulos, Composite Higgs scalars, Phys. Lett. 136B, 187 (1984).

[7] H. Georgi and D. B. Kaplan, Composite Higgs and custodial SU(2), Phys. Lett. 145B, 216 (1984).
[8] Y. Nambu, Quasiparticles and gauge invariance in the theory of superconductivity, Phys. Rev. 117, 648 (1960).

[9] S. Weinberg, Approximate Symmetries and PseudoGoldstone Bosons, Phys. Rev. Lett. 29, 1698 (1972).

[10] H. Georgi and A. Pais, Calculability and naturalness in gauge theories, Phys. Rev. D 10, 539 (1974).

[11] H. Georgi and A. Pais, Vacuum symmetry and the pseudoGoldstone phenomenon, Phys. Rev. D 12, 508 (1975).

[12] S. Weinberg, Implications of dynamical symmetry breaking, Phys. Rev. D 13, 974 (1976); Phys. Rev. D 19, 1277(A) (1979).

[13] R. Contino, The Higgs as a composite Nambu-Goldstone boson, in Theoretical Advanced Study Institute in Elementary Particle Physics: Physics of the Large and the Small (World Scientic Publishing, Singapore, 2011), pp. 235-306. 
[14] J. Mrazek, A. Pomarol, R. Rattazzi, M. Redi, J. Serra, and A. Wulzer, The other natural two Higgs doublet model, Nucl. Phys. B853, 1 (2011).

[15] B. Bellazzini, C. Csáki, and J. Serra, Composite Higgses, Eur. Phys. J. C 74, 2766 (2014).

[16] G. Panico and A. Wulzer, The Composite Nambu-Goldstone Higgs (Springer, New York, 2016), Vol. 913.

[17] K. Agashe, R. Contino, and A. Pomarol, The minimal composite Higgs model, Nucl. Phys. B719, 165 (2005).

[18] C. Csaki, A. Falkowski, and A. Weiler, The flavor of the composite pseudo-Goldstone Higgs, J. High Energy Phys. 09 (2008) 008.

[19] R. Contino, D. Marzocca, D. Pappadopulo, and R. Rattazzi, On the effect of resonances in composite Higgs phenomenology, J. High Energy Phys. 10 (2011) 081.

[20] G. von Gersdorff, E. Pontón, and R. Rosenfeld, The dynamical composite Higgs, J. High Energy Phys. 06 (2015) 119.

[21] E. Katz, A. E. Nelson, and D. G. E. Walker, The intermediate Higgs, J. High Energy Phys. 08 (2005) 074.

[22] M. Schmaltz and J. Thaler, Collective quartics and dangerous singlets in little Higgs, J. High Energy Phys. 03 (2009) 137.

[23] B. Gripaios, A. Pomarol, F. Riva, and J. Serra, Beyond the minimal composite Higgs model, J. High Energy Phys. 04 (2009) 070.

[24] M. Frigerio, A. Pomarol, F. Riva, and A. Urbano, Composite scalar dark matter, J. High Energy Phys. 07 (2012) 015 .

[25] M. Redi and A. Tesi, Implications of a light Higgs in composite models, J. High Energy Phys. 10 (2012) 166.

[26] D. Marzocca and A. Urbano, Composite dark matter and LHC interplay, J. High Energy Phys. 07 (2014) 107.

[27] S. R. Coleman, J. Wess, and B. Zumino, Structure of phenomenological Lagrangians. 1., Phys. Rev. 177, 2239 (1969).

[28] C. G. Callan, Jr., G. Curtis, S. R. Coleman, J. Wess, and B. Zumino, Structure of phenomenological Lagrangians. 2., Phys. Rev. 177, 2247 (1969).

[29] S. Weinberg, Phenomenological Lagrangians, Physica (Amsterdam) 96A, 327 (1979).

[30] A. Manohar and H. Georgi, Chiral quarks and the nonrelativistic quark model, Nucl. Phys. B234, 189 (1984).

[31] J. Gasser and H. Leutwyler, Chiral perturbation theory to one loop, Ann. Phys. (N.Y.) 158, 142 (1984).

[32] S. Weinberg, The Quantum Theory of Fields. Vol. 1: Foundations (Cambridge University Press, Cambridge, England, 2005).

[33] G. Buchalla, O. Catá, and C. Krause, On the power counting in effective field theories, Phys. Lett. B 731, 80 (2014).

[34] T. Appelquist and C. W. Bernard, Strongly interacting Higgs bosons, Phys. Rev. D 22, 200 (1980).

[35] A. C. Longhitano, Heavy Higgs bosons in the WeinbergSalam model, Phys. Rev. D 22, 1166 (1980).

[36] A. C. Longhitano, Low-energy impact of a heavy Higgs boson sector, Nucl. Phys. B188, 118 (1981).

[37] W. Buchmuller and D. Wyler, Effective lagrangian analysis of new interactions and flavor conservation, Nucl. Phys. B268, 621 (1986).
[38] T. Appelquist and G.-H. Wu, Electroweak chiral Lagrangian and new precision measurements, Phys. Rev. D 48, 3235 (1993).

[39] H. Leutwyler, On the foundations of chiral perturbation theory, Ann. Phys. (N.Y.) 235, 165 (1994).

[40] K. Hagiwara, S. Ishihara, R. Szalapski, and D. Zeppenfeld, Low-energy effects of new interactions in the electroweak boson sector, Phys. Rev. D 48, 2182 (1993).

[41] M. J. Herrero and E. R. Morales, The electroweak chiral Lagrangian for the standard model with a heavy Higgs, Nucl. Phys. B418, 431 (1994).

[42] G. F. Giudice, C. Grojean, A. Pomarol, and R. Rattazzi, The strongly-interacting light Higgs, J. High Energy Phys. 06 (2007) 045.

[43] B. Grzadkowski, M. Iskrzynski, M. Misiak, and J. Rosiek, Dimension-six terms in the standard model Lagrangian, J. High Energy Phys. 10 (2010) 085.

[44] G. Buchalla and O. Cata, Effective theory of a dynamically broken electroweak standard model at NLO, J. High Energy Phys. 07 (2012) 101.

[45] R. Alonso, M. B. Gavela, L. Merlo, S. Rigolin, and J. Yepes, The effective chiral Lagrangian for a light dynamical "Higgs Particle", Phys. Lett. B 722, 330 (2013); , Erratum, Phys. Lett. B 726, 926 (2013).

[46] G. Buchalla, O. Catà, and C. Krause, Complete electroweak chiral Lagrangian with a light Higgs at NLO, Nucl. Phys. B880, 552 (2014); Erratum, Nucl. Phys. B913, 475 (2016).

[47] I. Brivio, T. Corbett, O. J. P. Éboli, M. B. Gavela, J. Gonzalez-Fraile, M. C. Gonzalez-Garcia, L. Merlo, and S. Rigolin, Disentangling a dynamical Higgs, J. High Energy Phys. 03 (2014) 024.

[48] M. B. Gavela, J. Gonzalez-Fraile, M. C. Gonzalez-Garcia, L. Merlo, S. Rigolin, and J. Yepes, $C P$ violation with a dynamical Higgs, J. High Energy Phys. 10 (2014) 044.

[49] R. Alonso, I. Brivio, B. Gavela, L. Merlo, and S. Rigolin, Sigma decomposition, J. High Energy Phys. 12 (2014) 034.

[50] G. Buchalla, O. Cata, and C. Krause, A systematic approach to the SILH Lagrangian, Nucl. Phys. B894, 602 (2015).

[51] F.-K. Guo, P. Ruiz-Femenia, and J. J. Sanz-Cillero, One loop renormalization of the electroweak chiral Lagrangian with a light Higgs boson, Phys. Rev. D 92, 074005 (2015).

[52] I. M. Hierro, L. Merlo, and S. Rigolin, Sigma decomposition: The $C P$-odd Lagrangian, J. High Energy Phys. 04 (2016) 016.

[53] I. Brivio, J. Gonzalez-Fraile, M. C. Gonzalez-Garcia, and L. Merlo, The complete HEFT Lagrangian after the LHC Run I, Eur. Phys. J. C 76, 416 (2016).

[54] D. de Florian et al., Handbook of LHC Higgs cross sections: 4. Deciphering the nature of the Higgs sector (2017), Vol. 17. https://cds.cern.ch/record/2227475.

[55] A. Pich, I. Rosell, J. Santos, and J. J. Sanz-Cillero, Fingerprints of heavy scales in electroweak effective Lagrangians, J. High Energy Phys. 04 (2017) 012.

[56] R. Alonso, K. Kanshin, and S. Saa, Renormalization group evolution of Higgs effective field theory, Phys. Rev. D 97, 035010 (2018).

[57] G. Buchalla, O. Cata, A. Celis, M. Knecht, and C. Krause, Complete one-loop renormalization of the Higgs-electroweak chiral Lagrangian, Nucl. Phys. B928, 93 (2018). 
[58] R. Alonso, E. E. Jenkins, and A. V. Manohar, A geometric formulation of Higgs effective field theory: Measuring the curvature of scalar field space, Phys. Lett. B 754, 335 (2016).

[59] R. Alonso, E. E. Jenkins, and A. V. Manohar, Sigma models with negative curvature, Phys. Lett. B 756, 358 (2016).

[60] R. Alonso, E. E. Jenkins, and A. V. Manohar, Geometry of the scalar sector, J. High Energy Phys. 08 (2016) 101.

[61] R. Nagai, M. Tanabashi, K. Tsumura, and Y. Uchida, Symmetry and geometry in a generalized Higgs effective field theory: Finiteness of oblique corrections versus perturbative unitarity, Phys. Rev. D 100, 075020 (2019).

[62] Y. Hosotani, M. Tanaka, and N. Uekusa, $H$ parity and the stable Higgs boson in the $\mathrm{SO}(5) \times \mathrm{U}(1)$ gauge-Higgs unification, Phys. Rev. D 82, 115024 (2010).

[63] J. Pablo Hoyos Daza, Composite Higgs models, Ph.D. thesis, Sao Paulo University, 2019.

[64] I. Low, Minimally symmetric Higgs boson, Phys. Rev. D 91, 116005 (2015).

[65] M.E. Peskin and T. Takeuchi, Estimation of oblique electroweak corrections, Phys. Rev. D 46, 381 (1992).

[66] R. Barbieri, A. Pomarol, R. Rattazzi, and A. Strumia, Electroweak symmetry breaking after LEP-1 and LEP-2, Nucl. Phys. B703, 127 (2004).
[67] C. Grojean, W. Skiba, and J. Terning, Disguising the oblique parameters, Phys. Rev. D 73, 075008 (2006).

[68] K. Hagiwara, T. Hatsukano, S. Ishihara, and R. Szalapski, Probing nonstandard bosonic interactions via $\mathrm{W}$ boson pair production at lepton colliders, Nucl. Phys. B496, 66 (1997).

[69] I. Brivio, O. J. P. Éboli, M. B. Gavela, M. C. GonzalezGarcia, L. Merlo, and S. Rigolin, Higgs ultraviolet softening, J. High Energy Phys. 12 (2014) 004.

[70] K. Hagiwara, R. D. Peccei, D. Zeppenfeld, and K. Hikasa, Probing the weak boson sector in $e^{+} e^{-} \rightarrow W^{+} W^{-}$, Nucl. Phys. B282, 253 (1987).

[71] Y.-H. Qi, Y.-P. Kuang, B.-J. Liu, and B. Zhang, Anomalous gauge couplings of the Higgs boson at the CERN LHC: Semileptonic mode in WW scatterings, Phys. Rev. D 79, 055010 (2009); Erratum, Phys. Rev. D 82, 119902 (2010).

[72] H. Georgi, Lie Algebras in Particle Physics: From Isospin to Unified Theories (Westview Press, New York, 1999).

[73] R. Contino and M. Salvarezza, One-loop effects from spin-1 resonances in Composite Higgs models, J. High Energy Phys. 07 (2015) 065.

[74] A. S. Belyaev, O. J. P. Eboli, M. C. Gonzalez-Garcia, J. K. Mizukoshi, S. F. Novaes, and I. Zacharov, Strongly interacting vector bosons at the CERN LHC: Quartic anomalous couplings, Phys. Rev. D 59, 015022 (1998). 